\title{
Chemotherapy-associated liver injury
}

Citation for published version (APA):

Zhao, J. (2017). Chemotherapy-associated liver injury: mechanisms and impact on clinical outcome.

[Doctoral Thesis, Maastricht University]. Maastricht University. https://doi.org/10.26481/dis.20170908jz

Document status and date:

Published: 01/01/2017

DOI:

10.26481/dis.20170908jz

Document Version:

Publisher's PDF, also known as Version of record

\section{Please check the document version of this publication:}

- A submitted manuscript is the version of the article upon submission and before peer-review. There can be important differences between the submitted version and the official published version of record.

People interested in the research are advised to contact the author for the final version of the publication, or visit the DOI to the publisher's website.

- The final author version and the galley proof are versions of the publication after peer review.

- The final published version features the final layout of the paper including the volume, issue and page numbers.

Link to publication

\footnotetext{
General rights rights.

- You may freely distribute the URL identifying the publication in the public portal. please follow below link for the End User Agreement:

www.umlib.nl/taverne-license

Take down policy

If you believe that this document breaches copyright please contact us at:

repository@maastrichtuniversity.nl

providing details and we will investigate your claim.
}

Copyright and moral rights for the publications made accessible in the public portal are retained by the authors and/or other copyright owners and it is a condition of accessing publications that users recognise and abide by the legal requirements associated with these

- Users may download and print one copy of any publication from the public portal for the purpose of private study or research.

- You may not further distribute the material or use it for any profit-making activity or commercial gain

If the publication is distributed under the terms of Article $25 \mathrm{fa}$ of the Dutch Copyright Act, indicated by the "Taverne" license above, 


\title{
Chemotherapy-associated liver injury:
}

\author{
mechanisms and impact on clinical outcome
}


(C) Junfang Zhao, Maastricht 2017

No part of this book may be reproduced or transmitted in any form or by any means, without prior permission in writing by the author, or when appropriate, by the publishers of the publications.
Layout by:
Tiny Wouters
Cover design by:
Tomasz Karawajczyk
Cover photography by: Junfang Zhao
Printed by:
LT Imprenta, Rijswijk

ISBN: 978-90-9030503-5 


\section{Chemotherapy-associated liver injury: \\ mechanisms and impact on clinical outcome}

\section{Dissertation}

to obtain the degree of Doctor at Maastricht University, on the authority of Rector Magnificus,

Prof. dr. Rianne M. Letschert,

according to the decision of the Board of Deans,

to be defended in public on

Friday, September 8, 2017 at 12:00 hrs

by

Junfang Zhao, MD

赵俊芳

Born on June 18, 1986

in Henan, China 


\section{Supervisor}

Prof. dr. S.W.M. Olde Damink

Prof. dr. C.H.C. Dejong

\section{Co-supervisior}

Dr. S.S. Rensen

\section{Assessment Committee}

Prof. dr. R. Shiri-Sverdlov (Chairman)

Prof. dr. G. Beets (Netherlands Cancer Institute, Amsterdam)

Prof. dr. W. Buhre

Prof. dr. H. Grabsch

Prof. dr. P. Pessaux (University of Strasbourg, France) 


\section{Contents}

Chapter 1 General introduction, aims and outline of the thesis 7

Part I Influence of chemotherapy-associated liver injury on outcome after partial hepatectomy for colorectal liver metastases

Chapter 2 The influence of chemotherapy-associated sinusoidal dilatation on short-term outcome after partial hepatectomy for colorectal liver metastases: a systematic review with meta-analysis surg Oncol 2016; 25(3):298-307

Chapter 3 Systematic review of the influence of chemotherapy-associated liver injury on outcome after partial hepatectomy for colorectal liver metastases BrJ Surg 2017;104(8):990-1002

Chapter 4 Impact of chemotherapy-associated liver injury on postoperative infectious complications after partial hepatectomy for colorectal liver metastases Submitted

Chapter 5 Impact of chemotherapy-associated liver injury on tumour regression grade and survival after partial hepatectomy for colorectal liver metastases Submitted

Part II Mechanisms underlying chemotherapy-associated liver injury

Chapter 6 miR-21 and miR-150 downregulation is associated with oxaliplatininduced sinusoidal dilatation and impaired survival submitted

Chapter 7 Hepatic complement activation is associated with liver injury in patients with colorectal liver metastases

submitted

Chapter 8 Summary, implications, and future perspective

Valorisation addendum

Acknowledgements

Curriculum vitae

List of publications 



\section{Chapter 1}

General introduction, aims and outline of the thesis 
Chapter 1 


\section{Introduction}

By affecting over 1.3 million patients annually, ${ }^{1}$ colorectal cancer is the third most common cancer worldwide. Approximately half of these patients develop colorectal liver metastases (CRLM). ${ }^{2,3}$ Hepatic resection provides the best prospect of cure, but only up to 30 per cent of patients with liver metastases are initially eligible for hepatectomy.,4 Neoadjuvant chemotherapy may reduce tumour burden and facilitate future partial liver resection for those with tumours deemed unresectable., 5 sage of combinations of chemotherapeutic agents, such as oxaliplatin and irinotecan, has increased tumour response rates remarkably from 24 per cent to up to 70 per cent, compared to single treatment with 5-fluorouracil. ${ }^{7,8}$

The beneficial effect from systemic chemotherapy has been proven in randomized control trials. Despite that, the undesired liver parenchymal damage caused by chemotherapy, collectively referred to as chemotherapy-associated liver injury (CALI), is not negligible. For instance, oxaliplatin-based treatments increase the occurrence of sinusoidal obstruction syndrome $(\mathrm{SOS})^{9,10}$ and nodular regenerative hyperplasia, ${ }^{10-12}$ treatment with a combination of 5 -fluorouracil and leucovorin is associated with the development of steatosis, ${ }^{13}$ whereas irinotecan-based regimens appear to be related to steatohepatitis. ${ }^{14}$ Once occurred, CALI persists for a long period even after cessation of chemotherapy. Vigano et al. have recently shown that SOS and nodular regeneration may regress only after nine months without chemotherapy, whereas steatosis and steatohepatitis persist. $^{15}$

SOS, previously known as veno-occlusive disease, is a syndrome characterized by injury of the sinusoidal endothelial cells, sinusoidal dilatation, peliosis, venular lesions and fibrosis; sinusoidal dilatation is the hallmark feature of $\operatorname{SOS}^{9,10}$ A grading system developed by Rubbia-Brandt et al. allows pathologists to directly score sinusoidal dilatation, and is routinely used for stipulating severity of SOS. ${ }^{9,10}$ As a result of SOS, the hepatic microcirculation is disorganized with hyper-perfused zones intermingled with hypo-perfused zones, ${ }^{16}$ which causes regenerative hepatocyte plates intermingled with atrophic plates. Secondary to sinusoidal injury, nodular regeneration is likely a regenerative response to sinusoidal injury, and characterized by diffuse transformation of regular hepatic parenchyma into regenerative nodules, compressing the surrounding parenchyma leading to appearance of atrophic hepatocytic plates. ${ }^{10}$

Steatosis and steatohepatitis, which are characterized by dysregulation of hepatic fat accumulation with or without neutrophil infiltration, are also part of a broad range of 
liver injuries that may be caused by specific drugs. ${ }^{13,14}$ The grading system of Kleiner et al. distinguishes histological features of steatosis, lobular inflammation, hepatocellular ballooning, and fibrosis, allowing pathologists to quantify the severity of steatosis and steatohepatitis. ${ }^{17}$ Alternatively, a novel scoring system (steatosis, activity, and fibrosis, [SAF]) with supposedly reduced inter-observer variation has been recently proposed to evaluate severity of steatohepatitis. ${ }^{18}$

\section{CALI and short-term outcome after partial hepatectomy for CRLM}

An overview of research themes in the current thesis is presented in Figure 1.1 (dashed lines). The potential downstream side-effects of CALI on clinical outcome after partial hepatectomy are an active area of research surrounded with controversy. Despite certain studies claiming that the presence of CALI is associated with increased postoperative morbidity and mortality, ${ }^{19-24}$ others could not reproduce this. ${ }^{25-29}$ The presence of postoperative infectious complications, particularly surgical site infections, remains a crucial clinical concern that is associated with increased morbidity and mortality rates, ${ }^{30}$ prolonged length of hospital stay, ${ }^{31}$ increased healthcare cost, ${ }^{32,33}$ and even decreased long-term survival. ${ }^{30,34}$ Regardless of numerous advances made into practice to prevent infection, surgical site infections remain the second most common healthcare-related infection after respiratory tract infection. ${ }^{35}$ Nevertheless, our understanding of the impact of CALI on infectious complications after partial hepatectomy is poor, and the relation between CALI and postoperative morbidity and mortality remains unclear (Figure 1.1, blue symbols).

\section{CALI, tumour response, and long-term survival after partial hepatectomy for CRLM}

Apart from exploring the relation between CALI and postoperative short-term outcome, it is of importance to understand whether CALI has an impact on long-term survival. It has been shown by our group ${ }^{36}$ and subsequently by others ${ }^{37}$ that CALI is related to poor tumour response, which is expressed as tumour regression grade, to neoadjuvant chemotherapy (Figure 1.1, green symbols). Since CALI impairs tumour response, and a good tumour response facilitates future hepatic resection (the best cure for CRLM), ${ }^{5,6}$ it is reasonable to presume that the presence of CALI may be related to decreased survival. However, studies into this area are few. Although one study claims that 
chemotherapy-associated sinusoidal dilatation is associated with shortened survival, ${ }^{38}$ a second could not reproduce this. ${ }^{37}$

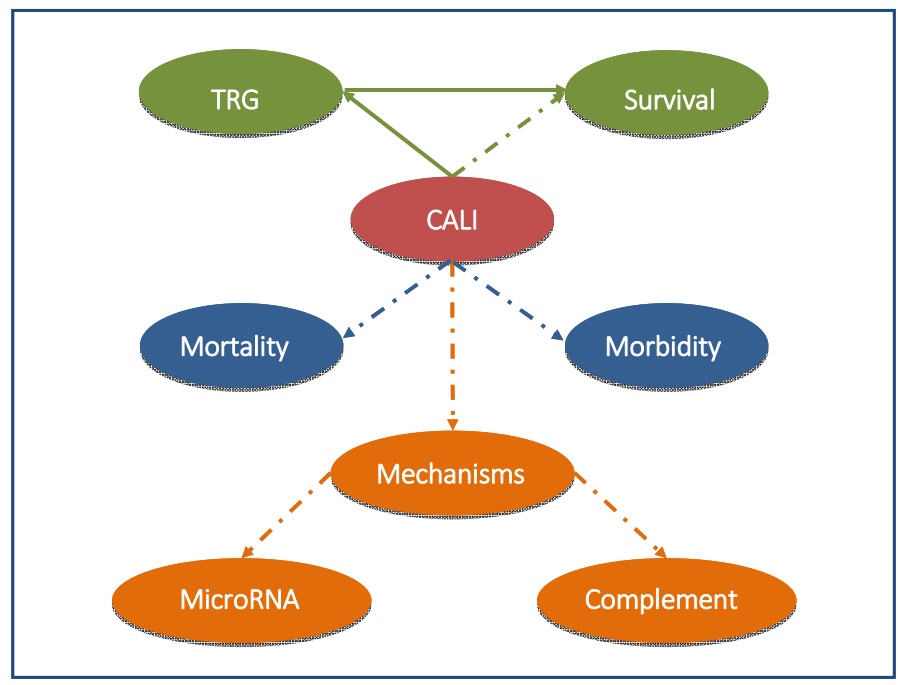

Figure 1.1 Research themes regarding chemotherapy-associated liver injury in this thesis.

TRG, tumour regression grade; CALI, chemotherapy-associated liver injury; solid lines indicate that the relation between two connected components is known in literature; dashed lines indicate that the relation between two connected components is controversial or unknown in literature; arrows indicate a likely cause-effect relationship.

\section{MicroRNAs: regulators of gene expression in CALI?}

Although CALI is likely to be of significant clinical importance, there is a lack of biomarkers that predict its occurrence, and there are currently no options to treat CALI. Furthermore, the molecular mechanisms underlying its development are still mostly unknown. These gaps in our current knowledge might be filled by studying the link between CALI and so-called microRNAs (Figure 1.1, yellow symbols), small non-coding RNA molecules that function as regulators of gene expression through translational repression and/or mRNA cleavage. ${ }^{39}$ Indeed, the concentration of several microRNAs has been found to be enriched in the serum of patients with acetaminophen-induced liver injury. ${ }^{40}$ Furthermore, specific circulating microRNAs are known to represent reliable biomarkers for predicting tumour response to chemotherapy and prognosis. ${ }^{41,42}$ Importantly, treating hepatitis C by targeting a specific microRNA involved in its pathogenesis has been shown to be feasible and effective. ${ }^{43}$ 


\section{Complement activation: the potential role of inflammation in CALI}

The liver contains numerous innate and adaptive immune cells, and represents a critical component in immune surveillance. ${ }^{44}$ As an important element of the innate immune system, the complement system mediates the regulation of immune responses to tissue injury and the initiation of inflammatory reactions. ${ }^{45}$ Pathophysiological processes leading to CALI involve an activation of the hepatic acute phase response. ${ }^{46}$ The acute phase response comprises a series of prompt immune responses to tissue injury, ${ }^{47}$ and may include activation of the complement pathways. ${ }^{48,49}$ Hepatic parenchymal injury as occurs in CALI may lead to the formation of C3a, which is cleaved from the central complement factor $\mathrm{C} 3$, and which promotes inflammation by recruiting neutrophils. The recruitment of these neutrophils has been shown to be essential for the development of nonalcoholic steatohepatitis, another form of sterile liver damage. ${ }^{50}$ Furthermore, C3a is rapidly cleaved by carboxypeptidases to generate acylation stimulating protein, a peptide that regulates triglyceride metabolism. ${ }^{51}$ As stated above, excessive accumulation of triglycerides in hepatocytes, or hepatic steatosis, ${ }^{52}$ is another important component of CALI and a central feature of steatohepatitis. Continued complement activation may result in the formation of so-called C5 convertase, which cleaves complement factor $\mathrm{C} 5$ into $\mathrm{C} 5 \mathrm{~b}$ and $\mathrm{C} 5 \mathrm{a}$. Apart from promoting inflammation via attracting circulating neutrophils into the liver, ${ }^{53} \mathrm{C} 5 \mathrm{a}$ has been suggested to regulate hepatic fibrosis by stimulating hepatic stellate cells ${ }^{54-56}$ and to play a role in the early stage of liver regeneration. ${ }^{57,58}$ Given that these (patho)physiological processes frequently occur in CALI, complement activation could play a role in its development (Figure 1.1, yellow symbols).

\section{Aims of this thesis}

The main aims of the current thesis were: 1 ) to explore whether CALI (i.e. sinusoidal dilatation, nodular regeneration, steatosis, and steatohepatitis) is a risk factor for increased morbidity (e.g. liver surgery-specific morbidity and major morbidity) and mortality, as well as infectious complications after partial hepatectomy for colorectal liver metastases; 2 ) to validate the relation between CALI and the tumour response to treatment, and further explore whether CALI is associated with survival after partial hepatectomy for CRLM; 3) to obtain novel insight into the pathogenesis of CALI, in particular sinusoidal injury, by identifying hepatic microRNAs associated with the development of such lesions; 4) to study the links between these microRNAs and tumour 
recurrence as well as survival; 5) to investigate whether complement activation plays a role in the development of CALI in patients undergoing partial hepatectomy for CRLM.

\section{Outline of this thesis}

In Part I, the impact of CALI (i.e. sinusoidal dilatation, nodular regeneration, steatosis, and steatohepatitis) on postoperative short-term (i.e. morbidity and mortality) and longterm (i.e. recurrence-free survival and overall survival) outcome is investigated. A systematic review with meta-analysis is described to study the impact of chemotherapyassociated sinusoidal dilatation on short-term outcome after partial hepatectomy for CRLM (Chapter 2). The existence of variations in study design and inclusion criteria, diverse definitions of a single endpoint, and varied endpoints under the category of morbidity as outlined in Chapter 2 raised the awareness that an unification for statistical modelling and a consensus definition for endpoints in CALI research is needed, and this led us to conduct a systematic review with individual participant data analyses on this topic (Chapter 3). Chapter 4 provides a detailed summary of the impact of CALI on postoperative infectious complications after partial hepatectomy for CRLM. Furthermore, the influence of CALI (i.e. sinusoidal dilatation, nodular regeneration, steatosis, and steatohepatitis) on tumour response to preoperative treatment, and thereafter on postoperative long-term outcome was explored. Chapter 5 describes a study on the influence of CALI on tumour response after neoadjuvant chemotherapy, and a summary of the association between CALI and long-term survival (i.e. recurrencefree survival and overall survival) after partial hepatectomy for CRLM.

In view of the clinical observations in Part I, and the lack of biomarkers to predict and options to treat CALI, Part II focuses on exploring the mechanisms underpinning CALI. Chapter 6 provides novel insights into the pathogenesis of CALI-related sinusoidal injury by identifying hepatic microRNAs associated with the development of such lesions. Chapter 7 describes the relation between CALI and hepatic complement activation in patients who had undergone partial hepatectomy for CRLM.

Chapter 8 summarizes the main findings as discussed in Part I and Part II of this thesis, provides a general discussion of those findings, and describes clinical implications and future perspectives of the research performed. 


\section{References}

1. Ferlay J, Soerjomataram I, Dikshit R, Eser S, Mathers C, Rebelo M, et al. Cancer incidence and mortality worldwide: sources, methods and major patterns in GLOBOCAN 2012. Int J Cancer 2015; 136(5):E359-86.

2. Leonard GD, Brenner B, Kemeny NE. Neoadjuvant chemotherapy before liver resection for patients with unresectable liver metastases from colorectal carcinoma. J Clin Oncol 2005; 23(9):2038-48.

3. Kanas GP, Taylor A, Primrose JN, Langeberg WJ, Kelsh MA, Mowat FS, et al. Survival after liver resection in metastatic colorectal cancer: review and meta-analysis of prognostic factors. Clin Epidemiol 2012; 4: 283-301.

4. Scheele J. Hepatectomy for liver metastases. Br J Surg 1993; 80(3):274-6.

5. Lam VW, Spiro C, Laurence JM, Johnston E, Hollands MJ, Pleass HC, et al. A systematic review of clinical response and survival outcomes of downsizing systemic chemotherapy and rescue liver surgery in patients with initially unresectable colorectal liver metastases. Ann Surg Oncol 2012; 19(4):1292-301.

6. Adam R, Delvart V, Pascal G, Valeanu A, Castaing D, Azoulay D, et al. Rescue surgery for unresectable colorectal liver metastases downstaged by chemotherapy: a model to predict long-term survival. Ann Surg 2004; 240(4):644-57; discussion 657-8.

7. Kalady MF, de Campos-Lobato LF, Stocchi L, Geisler DP, Dietz D, Lavery IC, et al. Predictive factors of pathologic complete response after neoadjuvant chemoradiation for rectal cancer. Ann Surg 2009; 250(4):582-9.

8. Folprecht G, Gruenberger T, Bechstein WO, Raab HR, Lordick F, Hartmann JT, et al. Tumour response and secondary resectability of colorectal liver metastases following neoadjuvant chemotherapy with cetuximab: the CELIM randomised phase 2 trial. Lancet Oncol 2010; 11(1):38-47.

9. Rubbia-Brandt L, Audard V, Sartoretti P, Roth AD, Brezault C, Le Charpentier M, et al. Severe hepatic sinusoidal obstruction associated with oxaliplatin-based chemotherapy in patients with metastatic colorectal cancer. Ann Oncol 2004; 15(3):460-6.

10. Rubbia-Brandt L, Lauwers GY, Wang H, Majno PE, Tanabe K, Zhu AX, et al. Sinusoidal obstruction syndrome and nodular regenerative hyperplasia are frequent oxaliplatin-associated liver lesions and partially prevented by bevacizumab in patients with hepatic colorectal metastasis. Histopathology 2010; 56(4):430-9.

11. Hubert C, Sempoux C, Horsmans Y, Rahier J, Humblet Y, Machiels JP, et al. Nodular regenerative hyperplasia: a deleterious consequence of chemotherapy for colorectal liver metastases? Liver Int 2007; 27(7):938-43.

12. Vigano L, Rubbia-Brandt L, De Rosa G, Majno P, Langella S, Toso C, et al. Nodular Regenerative Hyperplasia in Patients Undergoing Liver Resection for Colorectal Metastases After Chemotherapy: Risk Factors, Preoperative Assessment and Clinical Impact. Ann Surg Oncol 2015; 22(13):4149-57.

13. Khan AZ, Morris-Stiff G, Makuuchi M. Patterns of chemotherapy-induced hepatic injury and their implications for patients undergoing liver resection for colorectal liver metastases. J Hepatobiliary Pancreat Surg 2009; 16(2):137-44.

14. Robinson SM, Wilson $\mathrm{CH}$, Burt AD, Manas DM, White SA. Chemotherapy-associated liver injury in patients with colorectal liver metastases: a systematic review and meta-analysis. Ann Surg Oncol 2012; 19(13):4287-99.

15. Vigano L, De Rosa G, Toso C, Andres A, Ferrero A, Roth A, et al. Reversibility of chemotherapy-related liver injury. J Hepatol 2017.

16. DeLeve LD, Ito Y, Bethea NW, McCuskey MK, Wang X, McCuskey RS. Embolization by sinusoidal lining cells obstructs the microcirculation in rat sinusoidal obstruction syndrome. Am J Physiol Gastrointest Liver Physiol 2003; 284(6):G1045-52.

17. Kleiner DE, Brunt EM, Van Natta M, Behling C, Contos MJ, Cummings OW, et al. Design and validation of a histological scoring system for nonalcoholic fatty liver disease. Hepatology 2005; 41(6):1313-21.

18. Bedossa P, Consortium FP. Utility and appropriateness of the fatty liver inhibition of progression (FLIP) algorithm and steatosis, activity, and fibrosis (SAF) score in the evaluation of biopsies of nonalcoholic fatty liver disease. Hepatology 2014; 60(2):565-75. 
19. Aloia T, Sebagh M, Plasse M, Karam V, Levi F, Giacchetti S, et al. Liver histology and surgical outcomes after preoperative chemotherapy with fluorouracil plus oxaliplatin in colorectal cancer liver metastases. J Clin Oncol 2006; 24(31):4983-90.

20. Vauthey JN, Pawlik TM, Ribero D, Wu TT, Zorzi D, Hoff PM, et al. Chemotherapy regimen predicts steatohepatitis and an increase in 90-day mortality after surgery for hepatic colorectal metastases. J Clin Oncol 2006; 24(13):2065-72.

21. Nakano H, Oussoultzoglou E, Rosso E, Casnedi S, Chenard-Neu MP, Dufour P, et al. Sinusoidal injury increases morbidity after major hepatectomy in patients with colorectal liver metastases receiving preoperative chemotherapy. Ann Surg 2008; 247(1):118-24.

22. Pilgrim CH, Satgunaseelan L, Pham A, Murray W, Link E, Smith M, et al. Correlations between histopathological diagnosis of chemotherapy-induced hepatic injury, clinical features, and perioperative morbidity. HPB (Oxford) 2012; 14(5):333-40.

23. Vigano L, Ravarino N, Ferrero A, Motta M, Torchio B, Capussotti L. Prospective evaluation of accuracy of liver biopsy findings in the identification of chemotherapy-associated liver injuries. Arch Surg 2012; 147(12):1085-91.

24. Zorzi D, Laurent A, Pawlik TM, Lauwers GY, Vauthey JN, Abdalla EK. Chemotherapy-associated hepatotoxicity and surgery for colorectal liver metastases. Br J Surg 2007; 94(3):274-86.

25. Makowiec F, Mohrle S, Neeff H, Drognitz O, Illerhaus G, Opitz OG, et al. Chemotherapy, liver injury, and postoperative complications in colorectal liver metastases. J Gastrointest Surg 2011; 15(1):153-64.

26. Komori H, Beppu T, Baba Y, Horino K, Imsung C, Masuda T, et al. Histological liver injury and surgical outcome after FOLFOX followed by a hepatectomy for colorectal liver metastases in Japanese patients. Int J Clin Oncol 2010; 15(3):263-70.

27. Pawlik TM, Olino K, Gleisner AL, Torbenson M, Schulick R, Choti MA. Preoperative chemotherapy for colorectal liver metastases: impact on hepatic histology and postoperative outcome. J Gastrointest Surg 2007; 11(7):860-8.

28. Sahajpal A, Vollmer CM, Jr., Dixon E, Chan EK, Wei A, Cattral MS, et al. Chemotherapy for colorectal cancer prior to liver resection for colorectal cancer hepatic metastases does not adversely affect perioperative outcomes. J Surg Oncol 2007; 95(1):22-7.

29. Kandutsch S, Klinger M, Hacker S, Wrba F, Gruenberger B, Gruenberger T. Patterns of hepatotoxicity after chemotherapy for colorectal cancer liver metastases. Eur J Surg Oncol 2008; 34(11):1231-6.

30. Khuri SF, Henderson WG, DePalma RG, Mosca C, Healey NA, Kumbhani DJ, et al. Determinants of longterm survival after major surgery and the adverse effect of postoperative complications. Ann Surg 2005; 242(3):326-41; discussion 341-3.

31. Pessaux $P$, van den Broek MA, Wu T, Olde Damink SW, Piardi T, Dejong $\mathrm{CH}$, et al. Identification and validation of risk factors for postoperative infectious complications following hepatectomy. J Gastrointest Surg 2013; 17(11):1907-16.

32. Jenks PJ, Laurent M, McQuarry S, Watkins R. Clinical and economic burden of surgical site infection (SSI) and predicted financial consequences of elimination of SSI from an English hospital. J Hosp Infect 2014; 86(1):24-33.

33. Perencevich EN, Sands KE, Cosgrove SE, Guadagnoli E, Meara E, Platt R. Health and economic impact of surgical site infections diagnosed after hospital discharge. Emerg Infect Dis 2003; 9(2):196-203.

34. Artinyan A, Orcutt ST, Anaya DA, Richardson P, Chen GJ, Berger DH. Infectious postoperative complications decrease long-term survival in patients undergoing curative surgery for colorectal cancer: a study of 12,075 patients. Ann Surg 2015; 261(3):497-505.

35. Ploegmakers IB, Olde Damink SW, Breukink SO. Alternatives to antibiotics for prevention of surgical infection. Br J Surg 2017; 104(2):e24-e33.

36. Vreuls CP, Van Den Broek MA, Winstanley A, Koek GH, Wisse E, Dejong CH, et al. Hepatic sinusoidal obstruction syndrome (SOS) reduces the effect of oxaliplatin in colorectal liver metastases. Histopathology 2012; 61(2):314-8.

37. Vigano L, Capussotti L, De Rosa G, De Saussure WO, Mentha G, Rubbia-Brandt L. Liver resection for colorectal metastases after chemotherapy: impact of chemotherapy-related liver injuries, pathological tumor response, and micrometastases on long-term survival. Ann Surg 2013; 258(5):731-40; discussion $741-2$. 
38. Tamandl D, Klinger M, Eipeldauer S, Herberger B, Kaczirek K, Gruenberger B, et al. Sinusoidal obstruction syndrome impairs long-term outcome of colorectal liver metastases treated with resection after neoadjuvant chemotherapy. Ann Surg Oncol 2011; 18(2):421-30.

39. Rupaimoole R, Slack FJ. MicroRNA therapeutics: towards a new era for the management of cancer and other diseases. Nat Rev Drug Discov 2017; 16(3):203-222.

40. Krauskopf J, Caiment F, Claessen SM, Johnson KJ, Warner RL, Schomaker SJ, et al. Application of highthroughput sequencing to circulating microRNAs reveals novel biomarkers for drug-induced liver injury. Toxicol Sci 2015; 143(2):268-76.

41. Menendez P, Padilla D, Villarejo P, Palomino T, Nieto P, Menendez JM, et al. Prognostic implications of serum microRNA-21 in colorectal cancer. J Surg Oncol 2013; 108(6):369-73.

42. Toiyama Y, Takahashi M, Hur K, Nagasaka T, Tanaka K, Inoue Y, et al. Serum miR-21 as a diagnostic and prognostic biomarker in colorectal cancer. J Natl Cancer Inst 2013; 105(12):849-59.

43. Janssen HL, Reesink HW, Lawitz EJ, Zeuzem S, Rodriguez-Torres M, Patel K, et al. Treatment of HCV infection by targeting microRNA. N Engl J Med 2013; 368(18):1685-94.

44. Jenne CN, Kubes P. Immune surveillance by the liver. Nat Immunol 2013; 14(10):996-1006.

45. Bajic G, Degn SE, Thiel S, Andersen GR. Complement activation, regulation, and molecular basis for complement-related diseases. EMBO J 2015; 34(22):2735-57.

46. Rubbia-Brandt L, Tauzin S, Brezault C, Delucinge-Vivier C, Descombes P, Dousset B, et al. Gene expression profiling provides insights into pathways of oxaliplatin-related sinusoidal obstruction syndrome in humans. Mol Cancer Ther 2011; 10(4):687-96.

47. Baumann H, Gauldie J. The acute phase response. Immunol Today 1994; 15(2):74-80.

48. Du Clos TW. Function of C-reactive protein. Ann Med 2000; 32(4):274-8.

49. Mortensen RF. C-reactive protein, inflammation, and innate immunity. Immunol Res 2001; 24(2):163-76.

50. Rensen SS, Slaats Y, Driessen A, Peutz-Kootstra CJ, Nijhuis J, Steffensen R, et al. Activation of the complement system in human nonalcoholic fatty liver disease. Hepatology 2009; 50(6):1809-17.

51. Cianflone K, Vu H, Walsh M, Baldo A, Sniderman A. Metabolic response of Acylation Stimulating Protein to an oral fat load. J Lipid Res 1989; 30(11):1727-33.

52. Mazzoccoli G, Vinciguerra M, Oben J, Tarquini R, De Cosmo S. Non-alcoholic fatty liver disease: the role of nuclear receptors and circadian rhythmicity. Liver Int 2014; 34(8):1133-52.

53. Ricklin D, Hajishengallis G, Yang K, Lambris JD. Complement: a key system for immune surveillance and homeostasis. Nat Immunol 2010; 11(9):785-97.

54. Xu R, Lin F, He J, Jin L, Zhang JY, Fu J, et al. Complement 5a stimulates hepatic stellate cells in vitro, and is increased in the plasma of patients with chronic hepatitis B. Immunology 2013; 138(3):228-34.

55. Schlaf G, Schmitz M, Heine I, Demberg T, Schieferdecker HL, Gotze O. Upregulation of fibronectin but not of entactin, collagen IV and smooth muscle actin by anaphylatoxin C5a in rat hepatic stellate cells. Histol Histopathol 2004; 19(4):1165-74.

56. Hillebrandt S, Wasmuth HE, Weiskirchen R, Hellerbrand C, Keppeler H, Werth A, et al. Complement factor 5 is a quantitative trait gene that modifies liver fibrogenesis in mice and humans. Nat Genet 2005; 37(8):835-43.

57. Mastellos D, Papadimitriou JC, Franchini S, Tsonis PA, Lambris JD. A novel role of complement: mice deficient in the fifth component of complement (C5) exhibit impaired liver regeneration. J Immuno/ 2001; 166(4):2479-86.

58. Strey CW, Markiewski M, Mastellos D, Tudoran R, Spruce LA, Greenbaum LE, et al. The proinflammatory mediators C3a and C5a are essential for liver regeneration. J Exp Med 2003; 198(6):913-23. 


\section{Part I}

Influence of chemotherapy-associated liver injury on outcome after partial hepatectomy for colorectal liver metastases 



\section{Chapter}



The influence of chemotherapy-associated sinusoidal dilatation on short-term outcome after partial hepatectomy for colorectal liver metastases: a systematic review with meta-analysis 


\section{Abstract}

\section{Background}

Hepatic sinusoidal dilatation (SD) is a histopathological entity that occurs in up to $75 \%$ of patients undergoing oxaliplatin-based chemotherapy for colorectal liver metastases (CRLM). The aim was to study the influence of SD on outcome after partial hepatectomy in patients with CRLM.

\section{Methods}

Medline, Embase, CENTRAL, LILACS and CINAHL were searched for studies published between 01.01.2004 and 09.06.2015 with keywords: "sinusoidal obstruction syndrome", "hepatic veno-occlusive disease", and "Stuart-Bras syndrome". Studies comprising adults who underwent partial hepatectomy for CRLM with grading of SD and registration of postoperative morbidity and/or mortality were included. Risk of bias and quality of studies were evaluated with the Quality In Prognosis Studies Instrument (QUIPS) and modified GRADE framework.

\section{Results}

Search strategies produced 2007 hits from which 23 and 13 articles were extracted for qualitative and quantitative analyses, respectively. Meta-analysis on the influence of SD grade 2-3 vs. SD grade 0-1 on postoperative overall morbidity showed an odds ratio (OR) of 1.26 (95\% Cl 0.74-2.15, p=0.40, an OR of 1.03 (95\% Cl 0.15-6.89, $p=0.98$ ) for liver failure, an OR of 1.21 (95\% Cl 0.23-6.35, $\mathrm{p}=0.82$ ) for overall mortality, and an OR of 3.52 (95\% Cl 0.31-39.91, $p=0.31$ ) for liver-related morbidity. QUIPS showed a low to high risk of bias for studies, and GRADE showed very low quality of evidence per outcome.

\section{Conclusions}

No significant effect of SD grade 2-3 on short-term outcome after partial hepatectomy was found. However, the data on which this conclusion was based were not very robust and therefore no solid conclusions could be drawn. 


\section{Introduction}

Sinusoidal dilatation (SD) is a common manifestation of hepatotoxicity that occurs in patients with colorectal liver metastases (CRLM) after administration of oxaliplatin-based chemotherapy. ${ }^{1-3}$ Regimens based on the platinum containing agent oxaliplatin are used extensively as neo-adjuvant therapy to downsize initially irresectable CRLM, with convincing response rates and survival outcomes. ${ }^{4-6}$ However, liver injury is demonstrated in over $75 \%$ of patients. ${ }^{1,3}$

SD is part of a broad range of liver injuries due to specific drugs, in conjunction with sinusoidal obstruction syndrome (SOS). SOS is macroscopically identified as 'blue liver' and microscopically characterized by injury of the sinusoidal endothelial cells (SECS), parenchymal lesions (e.g. SD and peliosis), venular lesions, and fibrosis. Various pathogenic factors have been described to contribute to these histopathological changes. $^{7}$ Key features of oxaliplatin-induced toxicity are its reaction with reduced glutathione and F-actin depolymerization, which results in rounding up and subsequent dehiscence of SECs and obstruction of sinusoidal blood flow leading to SD and erythrocyte extravasation. ${ }^{1,8-12}$ Activation of hepatic stellate cells (HSC) results in neodisposition of collagen bundles in the perisinusoidal space which, in combination with subendothelial fibroblast activation in the terminal hepatic vein, leads to fibrotic venular occlusion. In the last stage of SOS, dense perivenular fibrosis is observed and hepatic vein lumina can no longer be identified. ${ }^{13}$

The grading system of Rubbia-Brandt et al. classifies all histological features of SOS including SD, and is routinely used for stipulating severity., ${ }^{1,2}$ In most studies, SD functions as the standard for liver damage. Reversibility of sinusoidal injury is discussed widely, and although human and animal models show ceasing of pathological features at repeated hepatic resection, ${ }^{2,14}$ other studies show persistence or progression of lesions even after cessation of chemotherapy. ${ }^{15}$

Clinical importance of SD is reflected in the development of hepatomegaly, ascites, splenomegaly, thrombocytopenia, portal hypertension, and systemic elevation of liver enzymes. ${ }^{16-19}$ With regard to liver surgery, a diminished preoperative functional reserve, (transient) postoperative liver failure, higher morbidity rates and a longer hospital stay, as well as impairment of postoperative liver regeneration have been reported. ${ }^{20,21}$ Numerous studies have shown a negative influence of SD on postoperative outcome, ${ }^{20,22,23}$ yet others could not reproduce this. ${ }^{24-29}$ This systematic review with 
meta-analysis aimed to determine the influence of SD on short-term outcome after partial hepatectomy in patients with CRLM.

\section{Methods}

\section{Criteria for considering studies for this review}

An extensive protocol, which is available upon request, was written before the start of this study. This review was conducted and reported in compliance with the PRISMA and MOOSE guidelines, and followed the Cochrane protocol for prognostic factor reviews. ${ }^{30-32}$ Studies were considered eligible for inclusion in this review when they met the following criteria: (I) studies comprising adults ( $\geq 18$ years old) with CRLM, (II) who underwent minor or major partial hepatectomy, (III) with postoperative histological grading of SD in liver tissue distant from the tumour according to the scoring system of Rubbia-Brandt, (IV) and with registration of overall morbidity, liver-related morbidity, liver failure, or overall mortality ( $\leq 90$ days or in-hospital) after liver resection. Case reports, comments/editorials, published abstracts, and reviews were rejected, in addition to records not covering the subject or including non-adults (age <18 years). Cohort studies including patients who underwent liver surgery for malignancies other than CRLM were included albeit that this group comprised less than $30 \%$ of the total study population. No distinction was made between first and repeated resections, and studies with patients who underwent preoperative portal vein embolization (PVE) were allowed for inclusion since an effect of PVE on outcome in patients with SD was observed in a single study only. ${ }^{33}$

\section{Search methods for identification of studies}

Search strategies in international databases Medline, Embase, Cochrane Central Register of Controlled Trials (CENTRAL), Latin American and Caribbean Health Sciences Literature (LILACS) and Cumulative Index to Nursing and Allied Health Literature (CINAHL) were performed between 01.01.2004 and 09.06.2015 using the following keywords (adjusted to the relevant database and including synonyms): "sinusoidal obstruction syndrome", "hepatic veno-occlusive disease", and "Stuart-Bras syndrome". Publication date of the search strategy was set from January 2004 onwards because the widely used criterion for scoring SD from Rubbia-Brandt et al. was developed in this year. ${ }^{1}$ The Embase strategy was independently peer reviewed by a second information specialist using the Canadian Agency for Drugs and Technologies in Health (CADTH) checklist. ${ }^{34}$ No language 
restrictions or other limitations were applied. Details of the search strategy can be found in Appendix 2.1 (supplementary data).

\section{Data collection and analysis}

Records were downloaded in EndNote ${ }^{\circledR} \mathrm{X} 7$ and duplicates were automatically and manually removed. All abstracts were screened by two independent reviewers (KvM, JZ), and in the rare case of no consensus the abstract was considered for full-text scanning. One of the reviewers (KVM) screened citations of all full-text articles for additional records on the base of title or abstract. Previous research from the authors on this topic resulted in an extensive own library which was also checked for references. Records considered for full-text assessment were screened independently by two reviewers (KvM, JZ). Full-text articles were screened for inclusion in qualitative analysis and/or quantitative analysis. Articles without postoperative mortality in the study population were described qualitatively due to lack of contribution to quantitative analysis.

\section{Data extraction and definitions}

Data extraction was performed independently by two reviewers (KvM, JZ) using a data extraction form in Excel specifically created for this study. Any dissimilarity in data was discussed and solved by consensus. Inter-observer agreement was calculated with Cohen's kappa coefficient. All information on study design and characteristics, main outcomes and possible overlap in cohort data was recorded. SD was defined according to the grading system of Rubbia-Brandt et al. $^{2}$ In short; SD 0: absence of signs of SD; 1 : mild SD (centrilobular involvement limited to one-third of the lobular area); 2: moderate SD (centrilobular involvement extending in two-thirds of the lobular area) and 3: severe SD (complete lobular involvement or centrilobular involvement extending to adjacent lobules with bridging congestion). In all analyses, 'SD' was defined as the presence of grade 2 or 3 SD whilst 'no SD' was defined as grade 0 or 1 . We considered SD grade 2-3 clinically more relevant than grade $0-1$, since rupture of sinusoidal wall integrity is present in grade 2 and higher. Major liver resections were defined as resection of $\geq 3$ Couinaud segments. All outcomes were measured between 30 and 90 days after partial hepatectomy or during initial hospital admission ('in-hospital'). Primary outcomes were overall morbidity, liver failure, and overall mortality after liver resection. Secondary outcome was liver-related morbidity after partial hepatectomy. Overall morbidity was defined as any complication (i.e. surgical and medical, excluding death) after liver resection, irrespective of severity. ${ }^{35}$ Mortality was defined as death due to any cause after liver resection. Since consensus on the definition of liver failure is lacking, ${ }^{36-38}$ definitions were specified in the results section. Liver-related morbidity was defined as 
any liver-related complication (e.g. bile leakage, liver abscess, liver failure) occurring after partial hepatectomy, regardless of severity. ${ }^{39}$

\section{Quality assessment}

Risk of bias of individual studies was determined using the Quality In Prognosis Studies (QUIPS) Risk of Bias Assessment Instrument for Prognostic Factor Studies. ${ }^{40,41}$ The QUIPS instrument is a checklist composed of the domains study participation, study attrition, prognostic factor measurement, outcome measurement, study confounding, and statistical analysis and reporting, and produces an estimation of the risk of bias with help of a rating (high, moderate or low risk) per domain. The following definitions were chosen by the authors for rating the overall risk of bias: 'overall low risk of bias' was $\leq 2$ domains rated as moderate risk and the remaining domains as low, 'overall moderate risk of bias' was $\geq 3$ domains rated as moderate risk and the remaining domains as low, 'overall high risk of bias' was $\geq 1$ domain rated as high risk, independent of the rating of the remaining domains. The quality of evidence per primary or secondary outcome was evaluated using the modified Grading of Recommendations Assessment, Development and Evaluation (GRADE) framework for prognosis studies, ${ }^{30,42}$ which is composed of eight scoring fields and aims to give an objective scoring of quality of evidence per outcome specific for the hypothesis of the user. Its fixed scoring parameters and rationale for downgrading described in the Summary of Findings Table makes GRADE the most transparent and reproducible method currently available. The starting score is based on the study design and quality can be downgraded (and in specific cases upgraded) per field. Quality of evidence is defined as (I) high quality: the true effect lies close to the estimate of the effect, (II) moderate quality: the true effect is likely to be close to the estimate of the effect, but there is a possibility that it is substantially different, (III) low quality: the true effect may be substantially different from the estimate of the effect or (IV) very low quality: the true effect is likely to be substantially different from the estimate of effect. ${ }^{30}$

\section{Data handling and statistical methods}

Meta-analyses of two or more studies per outcome were performed in Review Manager 5.3 and depicted in forest plots. The principal effect measures consisted of a pooled odds ratio $(\mathrm{OR})$ with $95 \%$ confidence intervals $(95 \% \mathrm{Cl})$ calculated by the MantelHaenszel test for dichotomous outcomes, which was chosen because of low event rates and small sample sizes. A p-value $<0.05$ was considered significant. A random effects model was chosen instead of fixed effects because the true effect size was expected to vary within different studies. Statistical heterogeneity was evaluated with the $X^{2}$ test, $I^{2}$, 
and comparison of point estimates between studies and overlap of $95 \%$ confidence intervals. Clinical heterogeneity was investigated by comparing different patient populations (SD 2-3 vs. SD 0-1; SD 1-3 vs. SD 0) and different extents of liver resection (all resections vs. major resections only). In case of significant heterogeneity, defined as an $I^{2} \geq 65 \%$ and a $X^{2}$ test $p$-value of $<0.10$ (considered significant due to a potentially low number of included studies in meta-analyses), the $\mathrm{OR}$ and $95 \% \mathrm{Cl}$ were omitted from the forest plot

\section{Results}

\section{Search results}

The conducted search resulted in a total of 2777 hits. Figure 2.1 shows the selection process of included studies in detail. After duplicate removal, 2007 hits remained. A total of 50 full-text articles were assessed for eligibility. Four more records were selected for full-text article scanning during reference checking, and another four potentially relevant records were selected from the own library. After application of the predefined inclusion and exclusion criteria on the total of 58 full-text articles by the two reviewers (KvM, JZ), 23 articles were included in qualitative synthesis. Inter-observer agreement kappa was 0.81 and dissimilarity was solved by discussion. Of the included studies for qualitative analysis, 13 studies contained data for quantitative analysis. Study and patient characteristics, main outcomes and main conclusions of the studies are summarized in Table 2.1.

\section{Included studies}

A total of eight studies could be included in quantitative analysis to estimate the effect of SD grade 2-3 vs. SD grade 0-1 on postoperative outcomes after partial hepatectomy. All studies but one were retrospective cohort studies which enrolled at least 50 patients. ${ }^{56}$ Each study was published in an international, peer-reviewed journal between 2006 and 2013. Postoperative morbidity could be evaluated in two studies comprising a total of 248 patients, ${ }^{26,44}$ and in two studies encompassing a total of 319 patients, postoperative liver failure was studied. ${ }^{47,56}$ Three studies investigated postoperative mortality in a total of 702 patients, ${ }^{21,55,56}$ and postoperative liver-related morbidity was evaluated in two studies with a total of 147 patients. ${ }^{22,26}$ For each of above outcomes, a single study addressed the effect of SD in patients undergoing major liver resection. ${ }^{26,53}$ 




Figure 2.1 Selection process of included studies.

This flowchart shows the exact steps that were taken from the search strategy until final inclusion of articles in qualitative and quantitative analysis, respectively. SD, sinusoidal dilatation; CINAHL, Cumulative Index to Nursing and Allied Health Literature; CENTRAL, Cochrane Central Register of Controlled Trials; LILACS, Latin American and Caribbean Health Sciences Literature; CRLM, colorectal liver metastases 







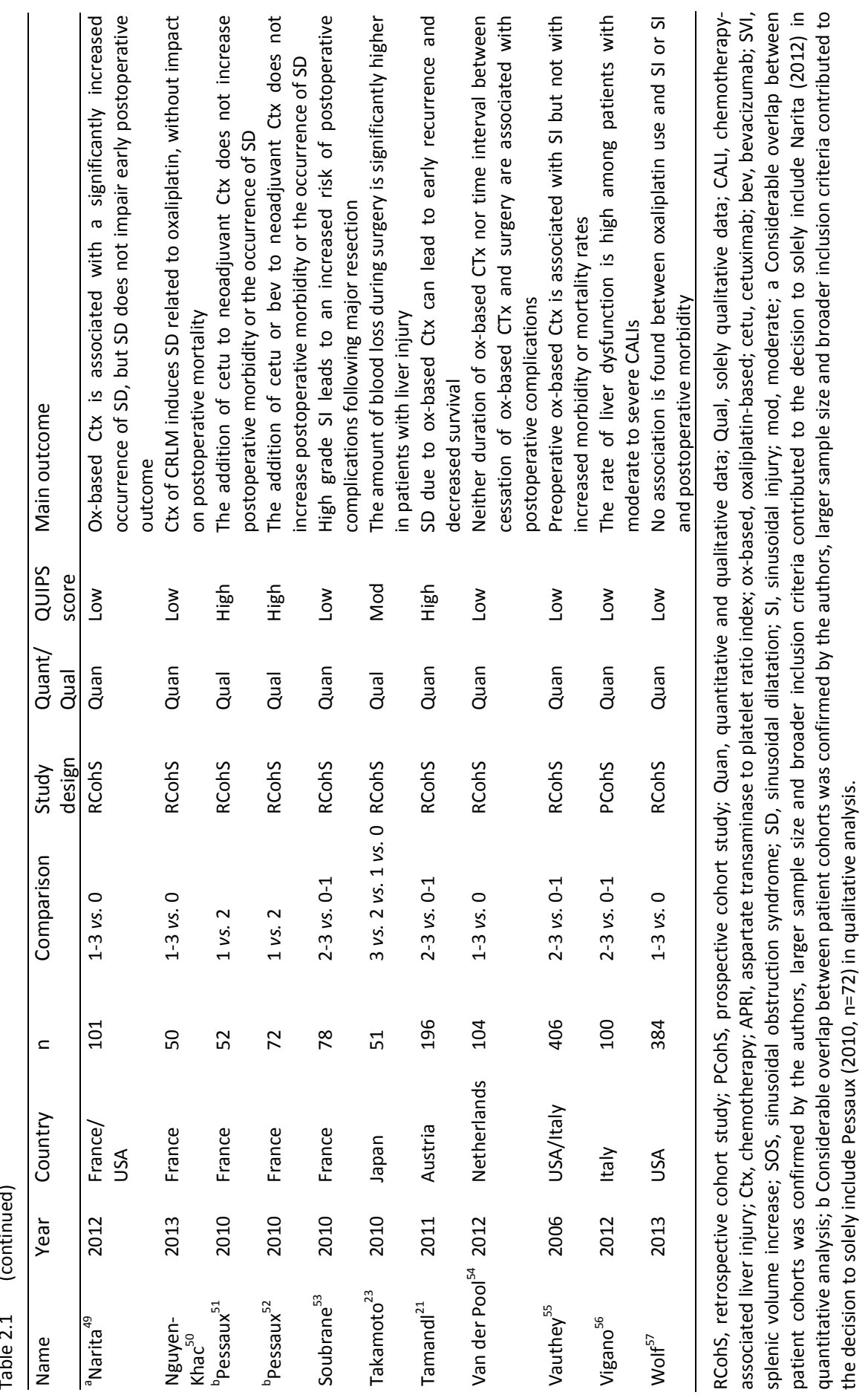




\section{Patient characteristics}

All cohort studies included patients who underwent minor or major hepatectomy and of whom liver quality was assessed postoperatively according to the grading system of Rubbia-Brandt. ${ }^{1}$ In total $63 \%$ of patients who underwent surgery were male, and median age was 61 years (range: 18 to 89). CRLM was the indication for liver surgery in all patients.

\section{Primary outcomes}

\section{Postoperative morbidity}

A meta-analysis on the influence of SD grade 2-3 vs. SD grade 0-1 on postoperative morbidity was conducted and depicted in Figure 2.2A. The overall effect of SD vs. no SD on postoperative morbidity showed a p-value of 0.40 with an OR of $1.26(95 \% \mathrm{Cl}$ $0.74-2.15)$. Tests evaluating consistency of results showed an $\mathrm{I}^{2}$ of $0 \%$ and a $\mathrm{X}^{2}$ test $p$-value of 0.74 . Although the use of random effects was deliberately chosen, testing with fixed effects did not have influence on the results $(O R$ 1.26, 95\% $\mathrm{Cl}$ 0.73-2.15, $\mathrm{p}=0.40$ ). SD was no risk factor for morbidity in studies where original data was not provided for quantitative analysis. ${ }^{21,24,50,55}$

In patients undergoing major hepatectomy ( $n=59$, hemihepatectomy or extended hemihepatectomy), 17 out of 29 patients (59\%) with SD developed morbidity vs. 19 out of 30 patients $(63 \%)$ in patients without SD (not depicted in forest plot). ${ }^{26}$

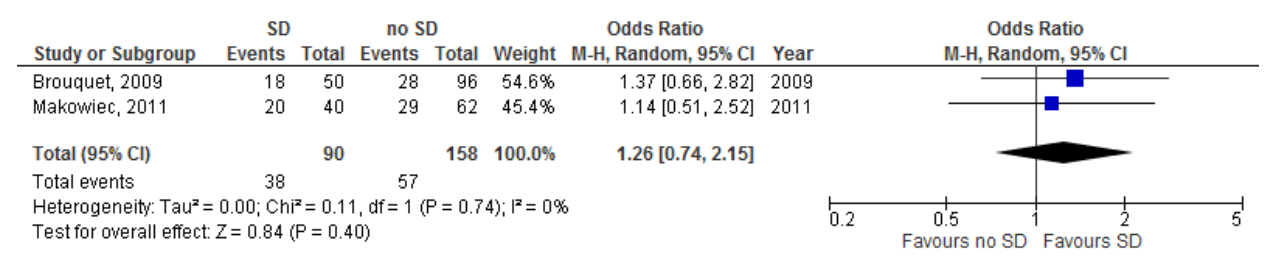

Figure 2.2a Postoperative overall morbidity.

Meta-analysis on the influence of sinusoidal dilatation (SD) grade 2-3 versus SD grade $0-1$ on the morbidity rate due to any cause after partial hepatectomy for colorectal liver metastases

Postoperative liver failure

The effect of SD on postoperative liver failure (PLF) could be tested in two studies (Figure 2.2B). In the study of Kishi et al., liver failure was defined as peak total bilirubin value $>120 \mathrm{\mu mol} / \mathrm{L}$ in the postoperative course, ${ }^{47}$ while Vigano et al. applied serum 
bilirubin $>50 \mu \mathrm{mol} / \mathrm{L}$ and/or prothrombin time $<50 \%$ on or after postoperative day (POD) $5 .^{56}$ An OR of 1.03 (95\% Cl 0.15-6.89) was found with a p-value of 0.98 for SD. Significant heterogeneity between studies was reflected in an $1^{2}$ of $65 \%$ and $p$-value of $x^{2}$ test of 0.09 , and overlap in $95 \% \mathrm{Cl}$ was barely present. Totals were subsequently omitted from the forest plot. One qualitative study showed no PLF in both the patients with or without SD. ${ }^{23}$ The study of Soubrane et al. was the only study that looked at postoperative liver failure in major resections only, and defined liver failure as a prothrombin time $<50 \%$ and serum bilirubin $>50 \mu \mathrm{mol} / \mathrm{L}$ on POD5. ${ }^{53}$ Liver failure occurred in eight of 38 patients with SD vs. zero in 13 patients without SD (not depicted in forest plot).

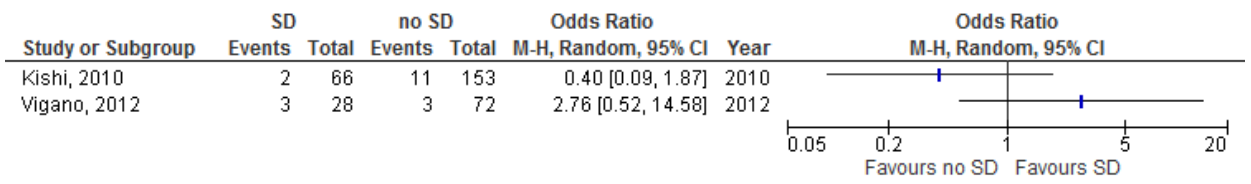

Figure 2.2b Postoperative liver failure.

Meta-analysis on the influence of sinusoidal dilatation (SD) grade 2-3 versus SD grade $0-1$ on the liver failure rate after partial hepatectomy for colorectal liver metastases.

\section{Postoperative mortality}

Three studies were available for examining the influence of SD on postoperative mortality (Figure 2.2C), with a total of 16 events. The test for overall effect showed a p-value of 0.82 with an OR of 1.21 (95\% Cl 0.23-6.35). Statistical testing for heterogeneity showed an $\mathrm{I}^{2}$ of $0 \%$ and $\mathrm{p}$-value of 0.79 . Assumption of fixed effects barely influenced the results (OR 1.15, 95\% Cl 0.24-5.51, p-value=0.86). Multiple studies described zero mortality in groups with or without SD, ${ }^{15,19,22-25,43,48,52}$ or no increase in mortality rate in patients with $\mathrm{SD}^{47}$.

\begin{tabular}{|c|c|c|c|c|c|c|c|c|c|c|}
\hline Study or Subgroup & \multicolumn{2}{|l|}{ SD } & \multicolumn{2}{|c|}{ no SD } & \multicolumn{3}{|c|}{ Odds Ratio } & \multicolumn{3}{|c|}{$\begin{array}{c}\text { Odds Ratio } \\
\text { M-H, Random, } 95 \% \mathrm{Cl}\end{array}$} \\
\hline Vauthey, 2006 & 0 & 22 & 11 & 384 & $33.6 \%$ & $0.72[0.04,12.64]$ & 2006 & & $\longrightarrow$ & \\
\hline Tamandl, 2011 & 0 & 21 & 4 & 175 & $31.5 \%$ & $0.89[0.05,17.03]$ & 2011 & & & \\
\hline Vigano, 2012 & 1 & 28 & 1 & 72 & $34.9 \%$ & $2.63[0.16,43.55]$ & 2012 & & & \\
\hline Total $(95 \% \mathrm{Cl})$ & & 71 & & 631 & $100.0 \%$ & $1.21[0.23,6.35]$ & & & & \\
\hline Total events & 1 & & 16 & & & & & & & \\
\hline $\begin{array}{l}\text { Heterogeneity: Tauz } \\
\text { Test for overall effect }\end{array}$ & $\begin{array}{l}0.00 ; \mathrm{Chi} \\
\mathrm{Z}=0.22(\end{array}$ & $\begin{array}{l}z=0.4 \\
P=0.8\end{array}$ & $\begin{array}{l}\text { 7, } d f=2(f \\
32)\end{array}$ & $P=0.7$ & $9) ;\left.\right|^{2}=0 \%$ & & & 0.02 & $\begin{array}{l}0.1 \\
\text { Favours no SD }\end{array}$ & ${ }^{1}$ Favours SD \\
\hline
\end{tabular}

Figure 2.2c Postoperative overall mortality.

Meta-analysis on the influence of sinusoidal dilatation (SD) grade 2-3 versus SD grade $0-1$ on the mortality rate due to any cause after partial hepatectomy for colorectal liver metastases. 
As described before, Soubrane et al. presented a subgroup analysis in patients who underwent major liver resection. ${ }^{53}$ In this study, two postoperative deaths occurred within 90 days in the SD group that consisted of 38 patients (4\%). Thirteen patients did not have SD with zero mortality.

\section{Secondary outcome}

\section{Liver-related morbidity}

Two studies were available for estimating the influence of SD on postoperative liverrelated morbidity (Figure 2.2D). The study of Gomez-Ramirez et al. included the following complications: biliary fistula, haemorrhage, abscesses, uninfected collections and liver failure, ${ }^{22}$ while Makowiec et al. included hepatic insufficiency, bilioma and/or symptomatic ascites requiring interventional or medical treatment. ${ }^{26}$ An OR of 3.52 (95\% $\mathrm{Cl}$ 0.31-39.91) was found with a $\mathrm{p}$-value of 0.31 for SD. $1^{2}$ was $83 \%$ with a $p$-value of $\mathrm{X}^{2}$ test of 0.01 . Overlapping of intervals between included studies was barely present; hence totals were omitted from the forest plot. In major hepatectomy only, eight out of 30 patients without SD vs. 10 out of 29 patients with SD developed liver-related complications. $^{26}$



Figure 2.2d Postoperative liver-related morbidity.

Meta-analysis on the influence of sinusoidal dilatation (SD) grade 2-3 versus SD grade $0-1$ on the liver-related morbidity rate after partial hepatectomy for colorectal liver metastases.

\section{Sub group analysis}

Five studies compared SD grade 1-3 with SD grade 0. Analyses on these subgroups were performed and resulting forest plots are presented in Appendix 2.2. In summary, the effect of SD grade 1-3 on the primary outcome postoperative morbidity could be tested in three studies $(n=577),{ }^{20,54,57}$ liver failure in one study $(n=90),{ }^{20}$ and mortality in two studies ( $n=433){ }^{50,57}$ Again, the risk for postoperative overall morbidity after minor or major liver resection in the SD group tended to be higher (OR 1.53, 95\% Cl 0.96-2.44, $\mathrm{p}=0.08$ ) than in the group without SD (Appendix 2.2A). There was limited evidence of effect (one study) of SD on PLF, and therefore no meta-analysis was conducted on this 
outcome. This study showed a higher incidence of PLF, which was defined as a serum bilirubin $>50 \mu \mathrm{mol} / \mathrm{L}$ and a prothrombin time $<50 \%$ on POD5 or thereafter, in the SD group (three out of 38 patients with SD vs. zero out of 52 patients without SD). ${ }^{20}$ Estimate points of effect for postoperative mortality resulted in an OR of 0.51 and $\mathrm{p}$-value of 0.54 (Appendix 2.2B). Statistical heterogeneity analysis revealed $\mathrm{I}^{2}=0 \%$ and $p$-values of 0.77 and 0.40 for postoperative overall morbidity and mortality, respectively. No mortality was observed in patient groups with and without SD in two studies. ${ }^{20,54}$ Secondary outcome measure liver-related morbidity could be tested in two studies ${ }^{20,57}$ and yielded an OR of $2.22(95 \% \mathrm{Cl}$ 0.34-14.32, $p=0.40)$ and an $\mathrm{I}^{2}$ of $64 \%(p=0.10)$ (Appendix 2.2C).

For major resections only, forest plots were constructed for postoperative overall morbidity and liver failure (Appendix 2.3). Two studies that included a total of 137 patients were available for a meta-analysis on postoperative morbidity (Appendix $2.3 \mathrm{~A}){ }^{20,49}$ An OR of 2.00 (95\% Cl 0.12-33.34) with a p-value of 0.63 for SD were found. $I^{2}$ was $83 \%$ with a $p$-value of $\mathrm{X}^{2}$ test of 0.02 , and totals were omitted from the forest plot. The same two studies were available for a meta-analysis on postoperative liver failure (Appendix 2.3B). Whereas Nakano et al. defined postoperative liver failure as a serum bilirubin $>50 \mu \mathrm{mol} / \mathrm{L}$ and a prothrombin time $<50 \%$ on or after $\mathrm{POD} 5,{ }^{20}$ Narita et al. defined this as a serum bilirubin $>50 \mu \mathrm{mol} / \mathrm{L}$ and a prothrombin time $<50 \%$ on POD 5 and/or a postoperative serum bilirubin $>120 \mu \mathrm{mol} / \mathrm{L} .{ }^{49} \mathrm{An}$ OR of 2.67 (95\% Cl 0.94-7.53) with a near-significant $p$-value of 0.06 for SD was found. No heterogeneity was detected as reflected in an $1^{2}$ of $0 \%$ with a $X^{2}$ test $p$-value of 0.73 . No mortality in patients with or without SD was observed in two studies on major hepatectomy. ${ }^{20,46}$ Liver-related morbidity was seen in the study of Nakano et al., ${ }^{20}$ with three patients showing liverrelated morbidity in the patient group with SD $(n=20)$ versus one patient in the patient group without SD $(n=16)$.

\section{Quality of the included studies}

\section{Risk of bias assessment}

Risk of bias was assessed using the modified QUIPS checklist ${ }^{40,41}$ and overall ratings are depicted in Table 2.1. Thirteen articles showed an overall low risk of bias, two studies showed moderate risk of bias and ten articles were appraised as having a high risk of bias. The assessment of risk of bias per domain and sub domain for individual studies can be found in Appendix 2.4. 


\section{GRADE assessment}

Study findings were assessed with the modified GRADE checklist ${ }^{42,58}$ and are shown in the Summary of Findings Table (Table 2.2). Footnotes provide detailed information about the rationale for downgrading. In short, evidence for all outcomes was rated as very low. Each outcome was downgraded on the base of study design (phase 1 explanatory studies). Other reasons for downgrading were indirectness in definition or time period of outcome measures and imprecision in data as shown by forest plots. Only QUIPS domain 1 to 4 were taken into account for downgrading, since domains 5 and 6 assess quality of the statistical analysis which did not influence quality of the current meta-analysis because we extracted only raw data from the manuscripts. A detailed rating per domain can be found in Appendix 2.5. A Summary of Findings Table for outcomes on SD grade 1-3 vs. 0 and a detailed rating per domain can be found in Appendix 2.6 and 2.7 .

\section{Discussion}

The present study found no significant influence of moderate to severe SD (grade 2-3) in comparison to no or mild SD (grade 0-1) on outcome after partial hepatectomy. Likewise, no influence on postoperative outcome was apparent when comparing mild to severe SD (grade 1-3) to no SD (grade 0). After data extraction, all studies were subjected to assessment with the QUIPS and GRADE tools to grade the quality of included evidence, strength of recommendations and risk of bias. Critical evaluation subsequently showed a low to high risk of bias for individual studies and very low quality of outcome-specific evidence, thereby leading to limited confidence in the provided evidence with regards to our hypothesis. An important explanation for less trust in the provided evidence is the study design of included articles. All but one study consisted of explanatory phase 1 studies, which are performed in early phases of investigation to generate a hypothesis and are considered weaker evidence than studies which confirm the independent effect of a specific prognostic factor on outcome (phase 2 studies), or studies that explore the underlying mechanism for prognosis of certain diseases (phase 3

studies). ${ }^{30,59}$ Moreover, variation in inclusion criteria, sample size differences, wide confidence intervals crossing the null value, and different definitions of outcomes, were factors that negatively affected the quality of the evidence specified for our hypothesis. In light of these findings, no solid conclusions can be drawn and this study cannot provide clinical advice on the topic. 


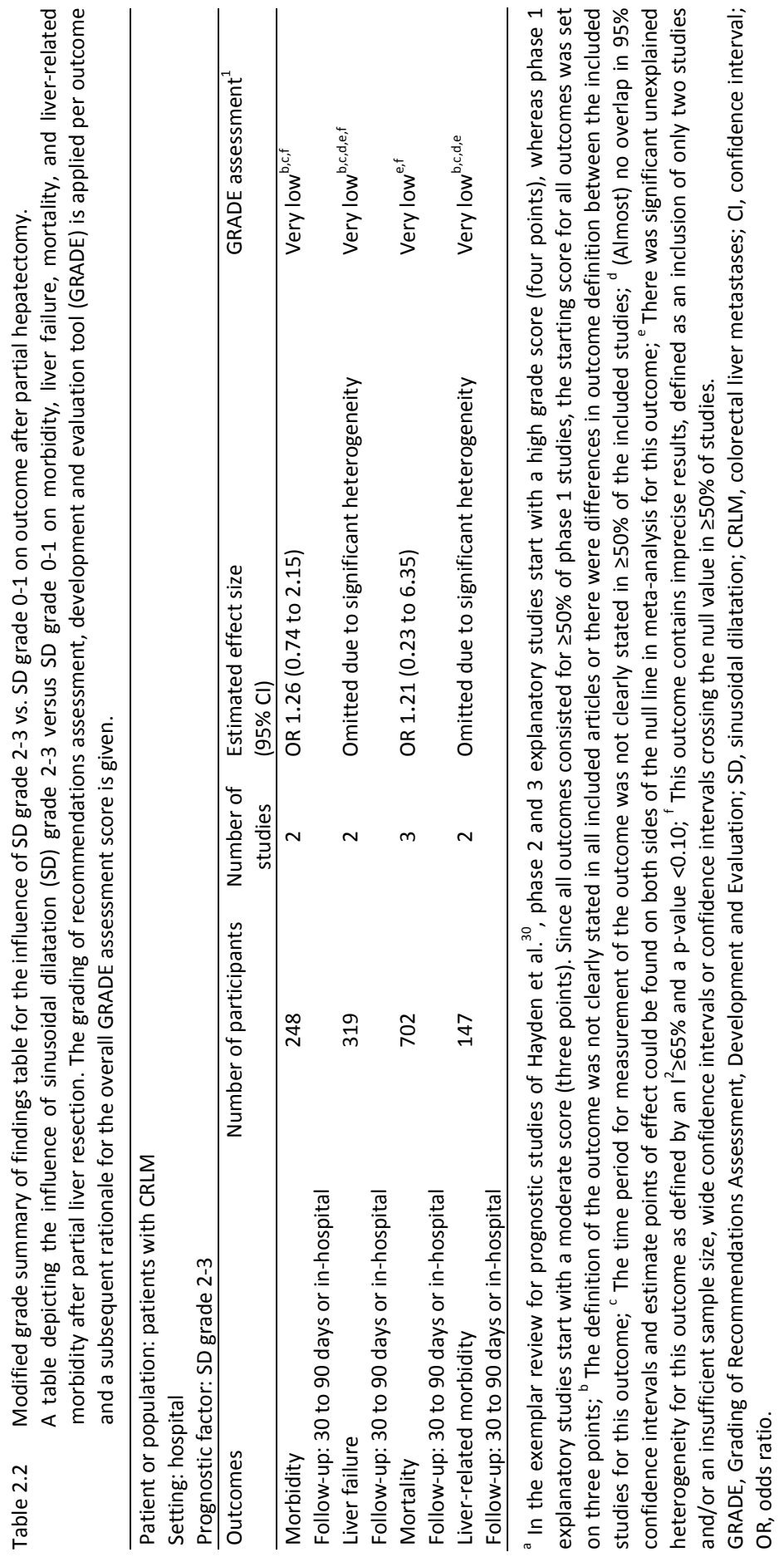


Some studies showed contrasting data, which may be partially explained by different inclusion criteria. While patients who received bevacizumab were intentionally excluded in some studies, ${ }^{26,50}$ other studies did allow inclusion of these patients ${ }^{22,47}$. Bevacizumab, an angiogenesis inhibitor that inhibits tumour growth by binding to vascular endothelial growth factor, is often co-administered in oxaliplatin-based chemotherapy regimens for patients with CRLM. ${ }^{60}$ Apart from the ongoing discussion about the potential benefit of prevention or reversibility of histological injury, ${ }^{2,61}$ bevacizumab itself may induce relevant toxic side effects which can affect recovery after partial liver resection. ${ }^{62-64}$ Moreover, it is usually advised to end chemotherapy, particularly when bevacizumab is co-administered, at least five weeks before liver surgery. ${ }^{65}$ Studies included in this systematic review report a range from 2 to 9 weeks for the interval between the last cycle of chemotherapy and surgery. It is currently unclear whether pathological characteristics of SOS are reversible, if a lower grade of SOS is linked to a higher likeliness of reversibility, and in which time frame this would occur. Patients with a long time interval between chemotherapy cessation and surgery were observed to have less SD compared to a short time interval, ${ }^{20}$ whereas irreversibility of SOS and even deterioration in time have been described by Mentha et al. ${ }^{15}$

Likewise, a link between the number of administered cycles of chemotherapy and grade of liver injury is uncertain, and the median number of administered cycles in this metaanalysis ranged from 6 to 12 . Whereas Karoui et al. found that the morbidity rate was correlated with the number of chemotherapy cycles, ${ }^{46}$ this correlation was not confirmed by Van Der Pool et al. who compared patients who received less than six cycles with those who received six or more cycles. ${ }^{54}$ One could speculate that patients with longer duration of chemotherapy may have had more extensive disease and therefore more complex surgical interventions. Almost all studies corrected for the extent of liver resection, but extrahepatic procedures and vascular reconstructions were not taken into account in more than half of studies. The impact of these procedures on outcome can be substantial. ${ }^{66,67}$

In patients with a critical future liver remnant due to anticipated extensive surgery, portal vein embolization (PVE) is the preferred procedure to induce pre-operative enlargement of the future liver remnant. ${ }^{68}$ The effect of SD on liver regeneration after PVE has been investigated in one study, in which PVE had a negative effect on postoperative liver regeneration. ${ }^{33}$ Moreover, post-PVE histopathological changes in a previous report were the rationale for Vauthey et al. ${ }^{55}$ and Brouquet et al. ${ }^{44}$ to exclude these patients from their cohorts. ${ }^{69}$ This resulted in a less complete reflection of the general surgical population. However, inclusion of these patients may have an impact on 
postoperative outcomes either by a diminished hypertrophic response or because this group of patients often undergoes extended hepatectomies and is already at higher risk.

With regard to variations in definitions, outcome after liver resection was expressed in numerous terms and time frames. Mortality was assessed within 30, 60, or 90 days after surgery or during hospital admission, and morbidity was described as overall, medical, surgical, liver-related or infectious, with no consensus on employed definitions. This underscores the necessity of a uniform outcome set for liver surgery in order to ensure clear and consistent clinically relevant data, and to allow comparisons between future trials and cohort studies in meta-analyses. ${ }^{39}$

On the basis of stated differences in inclusion criteria and outcome, which all might have influenced the outcome after liver resection to an unknown extent, ideally a phase two study in which all confounders are corrected for in a statistically appropriate way should be conducted. Our group recently initiated such a study in the form of an individual patient data meta-analysis. ${ }^{70}$

\section{Conclusion}

This study aimed to evaluate the influence of moderate to severe SD on outcome in patients undergoing partial hepatectomy for CRLM. Although many individual studies suggest a negative impact on postoperative (liver-related) morbidity, liver failure and mortality, the present meta-analysis could not confirm this data. However, trust in the obtained evidence was low and therefore no solid conclusions can be drawn. This study emphasizes the importance of critical risk of bias assessment and evaluation of quality in meta-analyses, to provide the most robust level of evidence for clinical decision making. It also highlights the need for unambiguous definitions of outcome in surgical oncology. 


\section{References}

1. Rubbia-Brandt L, Audard V, Sartoretti P, Roth AD, Brezault C, Le Charpentier M, et al. Severe hepatic sinusoidal obstruction associated with oxaliplatin-based chemotherapy in patients with metastatic colorectal cancer. Ann Oncol 2004; 15(3):460-6.

2. Rubbia-Brandt L, Lauwers GY, Wang H, Majno PE, Tanabe K, Zhu AX, et al. Sinusoidal obstruction syndrome and nodular regenerative hyperplasia are frequent oxaliplatin-associated liver lesions and partially prevented by bevacizumab in patients with hepatic colorectal metastasis. Histopathology 2010; 56(4):430-9.

3. Robinson $\mathrm{SM}$, Wilson $\mathrm{CH}$, Burt AD, Manas DM, White SA. Chemotherapy-associated liver injury in patients with colorectal liver metastases: a systematic review and meta-analysis. Ann Surg Oncol 2012; 19(13):4287-99.

4. Lam VW, Spiro C, Laurence JM, Johnston E, Hollands MJ, Pleass HC, et al. A systematic review of clinical response and survival outcomes of downsizing systemic chemotherapy and rescue liver surgery in patients with initially unresectable colorectal liver metastases. Ann Surg Oncol 2012; 19(4):1292-301.

5. Seium Y, Stupp R, Ruhstaller T, Gervaz P, Mentha G, Philippe M, et al. Oxaliplatin combined with irinotecan and 5-fluorouracil/leucovorin (OCFL) in metastatic colorectal cancer: a phase I-II study. Ann Oncol 2005; 16(5):762-6.

6. Adam R, Delvart V, Pascal G, Valeanu A, Castaing D, Azoulay D, et al. Rescue surgery for unresectable colorectal liver metastases downstaged by chemotherapy: a model to predict long-term survival. Ann Surg 2004; 240(4):644-57; discussion 657-8.

7. Agostini J, Benoist S, Seman M, Julie C, Imbeaud S, Letourneur F, et al. Identification of molecular pathways involved in oxaliplatin-associated sinusoidal dilatation. J Hepatol 2012; 56(4):869-76.

8. Nakamura K, Hatano E, Miyagawa-Hayashino A, Okuno M, Koyama Y, Narita M, et al. Soluble thrombomodulin attenuates sinusoidal obstruction syndrome in rat through suppression of high mobility group box 1. Liver Int 2014; 34(10):1473-87.

9. Robinson SM, Mann J, Vasilaki A, Mathers J, Burt AD, Oakley F, et al. Pathogenesis of FOLFOX induced sinusoidal obstruction syndrome in a murine chemotherapy model. J Hepatol 2013; 59(2):318-26.

10. Wang X, Kanel GC, DeLeve LD. Support of sinusoidal endothelial cell glutathione prevents hepatic venoocclusive disease in the rat. Hepatology 2000; 31(2):428-34.

11. Deleve LD, Ito Y, Bethea NW, McCuskey MK, Wang X, McCuskey RS. Embolization by sinusoidal lining cells obstructs the microcirculation in rat sinusoidal obstruction syndrome. Am J Physiol Gastrointest Liver Physiol 2003; 284(6):G1045-52.

12. Ezzat $\mathrm{T}$, van den Broek MA, Davies N, Dejong $\mathrm{CH}$, Bast A, Malago $\mathrm{M}$, et al. The flavonoid monoHER prevents monocrotaline-induced hepatic sinusoidal injury in rats. J Surg Oncol 2012; 106(1):72-8.

13. Fan CQ, Crawford JM. Sinusoidal obstruction syndrome (hepatic veno-occlusive disease). J Clin Exp Hepatol 2014; 4(4):332-46.

14. Deleve LD, McCuskey RS, Wang X, Hu L, McCuskey MK, Epstein RB, et al. Characterization of a reproducible rat model of hepatic veno-occlusive disease. Hepatology 1999; 29(6):1779-91.

15. Mentha G, Terraz S, Morel P, Andres A, Giostra E, Roth A, et al. Dangerous halo after neoadjuvant chemotherapy and two-step hepatectomy for colorectal liver metastases. Br J Surg 2009; 96(1):95-103.

16. Overman MJ, Maru DM, Charnsangavej C, Loyer EM, Wang H, Pathak P, et al. Oxaliplatin-mediated increase in spleen size as a biomarker for the development of hepatic sinusoidal injury. J Clin Oncol 2010; 28(15):2549-55.

17. Arotcarena R, Cales V, Berthelemy P, Parent Y, Malet M, Etcharry F, et al. Severe sinusoidal lesions: a serious and overlooked complication of oxaliplatin-containing chemotherapy? Gastroenterol Clin Biol 2006; 30(11):1313-6.

18. Slade JH, Alattar ML, Fogelman DR, Overman MJ, Agarwal A, Maru DM, et al. Portal hypertension associated with oxaliplatin administration: clinical manifestations of hepatic sinusoidal injury. Clin Colorectal Cancer 2009; 8(4):225-30.

19. Imai K, Emi Y, Iyama KI, Beppu T, Ogata Y, Kakeji Y, et al. Splenic volume may be a useful indicator of the protective effect of bevacizumab against oxaliplatin-induced hepatic sinusoidal obstruction syndrome. Eur J Surg Oncol 2014; 40(5):559-66. 
20. Nakano H, Oussoultzoglou E, Rosso E, Casnedi S, Chenard-Neu MP, Dufour P, et al. Sinusoidal injury increases morbidity after major hepatectomy in patients with colorectal liver metastases receiving preoperative chemotherapy. Ann Surg 2008; 247(1):118-24.

21. Tamandl D, Klinger M, Eipeldauer S, Herberger B, Kaczirek K, Gruenberger B, et al. Sinusoidal obstruction syndrome impairs long-term outcome of colorectal liver metastases treated with resection after neoadjuvant chemotherapy. Ann Surg Oncol 2011; 18(2):421-30.

22. Gomez-Ramirez J, Martin-Perez E, Amat CG, Sanz IG, Bermejo E, Rodriguez A, et al. [Influence of presurgical chemotherapy on liver parenchyma and post-surgical outcome of patients subjected to hepatectomy due to colorectal carcinoma metastases]. Cir Esp 2010; 88(6):404-12.

23. Takamoto T, Hashimoto T, Sano K, Maruyama Y, Inoue K, Ogata S, et al. Recovery of liver function after the cessation of preoperative chemotherapy for colorectal liver metastasis. Ann Surg Oncol 2010; 17(10):2747-55.

24. Kandutsch S, Klinger M, Hacker S, Wrba F, Gruenberger B, Gruenberger T. Patterns of hepatotoxicity after chemotherapy for colorectal cancer liver metastases. Eur J Surg Oncol 2008; 34(11):1231-6.

25. Komori H, Beppu T, Baba Y, Horino K, Imsung C, Masuda T, et al. Histological liver injury and surgical outcome after FOLFOX followed by a hepatectomy for colorectal liver metastases in Japanese patients. Int J Clin Oncol 2010; 15(3):263-270.

26. Makowiec F, Mohrle S, Neeff H, Drognitz O, Illerhaus G, Opitz OG, et al. Chemotherapy, liver injury, and postoperative complications in colorectal liver metastases. J Gastrointest Surg 2011; 15(1):153-64.

27. Pawlik TM, Olino K, Gleisner AL, Torbenson M, Schulick R, Choti MA. Preoperative chemotherapy for colorectal liver metastases: impact on hepatic histology and postoperative outcome. J Gastrointest Surg 2007; 11(7):860-868.

28. Sahajpal A, Vollmer CM, Dixon E, Chan EK, Wei A, Cattral MS, et al. Chemotherapy for colorectal cancer prior to liver resection for colorectal cancer hepatic metastases does not adversely affect peri-operative outcomes. J Surg Oncol 2007; 95(1):22-27.

29. Takeda D, Nitta H, Takahara T, Hasegawa Y, Itou N, Wakabayashi G. Effect of preoperative chemotherapy on postoperative liver regeneration following hepatic resection as estimated by liver volume. World J Surg Oncol 2013; 11:65.

30. Hayden JA, Tougas ME, Riley R, lles R, Pincus T. Individual recovery expectations and prognosis of outcomes in non-specific low back pain: prognostic factor exemplar review (Protocol). Cochrane Database of Systematic Reviews 2014(Issue 9).

31. Moher D, Liberati A, Tetzlaff J, Altman DG, Group P. Preferred reporting items for systematic reviews and meta-analyses: the PRISMA statement. Ann Intern Med 2009; 151(4):264-9, W64.

32. Stroup DF, Berlin JA, Morton SC, Olkin I, Williamson GD, Rennie D, et al. Meta-analysis of observational studies in epidemiology - A proposal for reporting. JAMA 2000; 283(15):2008-2012.

33. Narita M, Oussoultzoglou E, Chenard MP, Rosso E, Casnedi S, Pessaux P, et al. Sinusoidal obstruction syndrome compromises liver regeneration in patients undergoing two-stage hepatectomy with portal vein embolization. Surg Today 2011; 41(1):7-17.

34. CADTH. Canadian Agency for Drugs and Technologies in Health. CADTH peer review checklist for search strategies. 2013:3p.

35. Dindo D, Demartines N, Clavien PA. Classification of surgical complications: a new proposal with evaluation in a cohort of 6336 patients and results of a survey. Ann Surg 2004; 240(2):205-13.

36. Balzan S, Belghiti J, Farges O, Ogata S, Sauvanet A, Delefosse D, et al. The "50-50 criteria" on postoperative day 5: an accurate predictor of liver failure and death after hepatectomy. Ann Surg 2005; 242(6):824-8, discussion 828-9.

37. Mullen JT, Ribero D, Reddy SK, Donadon M, Zorzi D, Gautam S, et al. Hepatic insufficiency and mortality in 1,059 noncirrhotic patients undergoing major hepatectomy. J Am Coll Surg 2007; 204(5):854-62; discussion 862-4.

38. Rahbari NN, Garden OJ, Padbury R, Brooke-Smith M, Crawford M, Adam R, et al. Posthepatectomy liver failure: a definition and grading by the International Study Group of Liver Surgery (ISGLS). Surgery 2011; 149(5):713-24.

39. van den Broek MA, van Dam RM, van Breukelen GJ, Bemelmans MH, Oussoultzoglou E, Pessaux P, et al. Development of a composite endpoint for randomized controlled trials in liver surgery. Br J Surg 2011; 98(8):1138-45. 
40. Hayden JA, Cote $P$, Bombardier $C$. Evaluation of the quality of prognosis studies in systematic reviews. Ann Intern Med 2006; 144(6):427-37.

41. Hayden JA, van der Windt DA, Cartwright JL, Cote P, Bombardier C. Assessing bias in studies of prognostic factors. Ann Intern Med 2013; 158(4):280-6.

42. Davis P, Hayden J, Springer J, Bailey J, Molinari M, Johnson P. Prognostic factors for morbidity and mortality in elderly patients undergoing acute gastrointestinal surgery: a systematic review. Can J Surg 2014; 57(2):E44-52.

43. Aloysius MM, Zaitoun AM, Beckingham IJ, Neal KR, Aithal GP, Bessell EM, et al. The pathological response to neoadjuvant chemotherapy with FOLFOX-4 for colorectal liver metastases: a comparative study. Virchows Arch 2007; 451(5):943-8.

44. Brouquet A, Benoist S, Julie C, Penna C, Beauchet A, Rougier P, et al. Risk factors for chemotherapyassociated liver injuries: A multivariate analysis of a group of 146 patients with colorectal metastases. Surgery 2009; 145(4):362-71.

45. Hubert C, Fervaille C, Sempoux C, Horsmans Y, Humblet Y, Machiels JP, et al. Prevalence and clinical relevance of pathological hepatic changes occurring after neoadjuvant chemotherapy for colorectal liver metastases. Surgery 2010; 147(2):185-94.

46. Karoui M, Penna C, Amin-Hashem M, Mitry E, Benoist S, Franc B, et al. Influence of preoperative chemotherapy on the risk of major hepatectomy for colorectal liver metastases. Ann Surg 2006; 243(1): 1-7.

47. Kishi Y, Zorzi D, Contreras CM, Maru DM, Kopetz S, Ribero D, et al. Extended preoperative chemotherapy does not improve pathologic response and increases postoperative liver insufficiency after hepatic resection for colorectal liver metastases. Ann Surg Oncol 2010; 17(11):2870-6.

48. Miura K, Nakano H, Sakurai J, Kobayashi S, Koizumi S, Arai T, et al. Splenomegaly in FOLFOX-naive stage IV or recurrent colorectal cancer patients due to chemotherapy-associated hepatotoxicity can be predicted by the aspartate aminotransferase to platelet ratio before chemotherapy. Int J Clin Oncol 2011; 16(3):257-63.

49. Narita M, Oussoultzoglou E, Fuchshuber P, Pessaux P, Chenard MP, Rosso E, et al. What is a safe future liver remnant size in patients undergoing major hepatectomy for colorectal liver metastases and treated by intensive preoperative chemotherapy? Ann Surg Oncol 2012; 19(8):2526-38.

50. Nguyen-Khac E, Lobry C, Chatelain D, Fuks D, Joly JP, Brevet M, et al. A Reappraisal of ChemotherapyInduced Liver Injury in Colorectal Liver Metastases before the Era of Antiangiogenics. Int J Hepatol 2013; 2013:314868.

51. Pessaux P, Marzano E, Casnedi S, Bachellier P, Jaeck D, Chenard MP. Histological and immediate postoperative outcome after preoperative cetuximab: case-matched control study. World J Surg 2010; 34(11):2765-72.

52. Pessaux P, Panaro F, Casnedi S, Zeca I, Marzano E, Bachellier P, et al. Targeted molecular therapies (cetuximab and bevacizumab) do not induce additional hepatotoxicity: preliminary results of a casecontrol study. Eur J Surg Oncol 2010; 36(6):575-82.

53. Soubrane O, Brouquet A, Zalinski S, Terris B, Brezault C, Mallet V, et al. Predicting high grade lesions of sinusoidal obstruction syndrome related to oxaliplatin-based chemotherapy for colorectal liver metastases: correlation with post-hepatectomy outcome. Ann Surg 2010; 251(3):454-60.

54. van der Pool AE, Marsman HA, Verheij J, Ten Kate FJ, Eggermont AM, ljzermans JN, et al. Effect of bevacizumab added preoperatively to oxaliplatin on liver injury and complications after resection of colorectal liver metastases. J Surg Oncol 2012; 106(7):892-7.

55. Vauthey JN, Pawlik TM, Ribero D, Wu TT, Zorzi D, Hoff PM, et al. Chemotherapy regimen predicts steatohepatitis and an increase in 90-day mortality after surgery for hepatic colorectal metastases. J Clin Oncol 2006; 24(13):2065-72.

56. Vigano L, Ravarino N, Ferrero A, Motta M, Torchio B, Capussotti L. Prospective evaluation of accuracy of liver biopsy findings in the identification of chemotherapy-associated liver injuries. Arch Surg. 2012; 147(12):1085-91.

57. Wolf PS, Park JO, Bao F, Allen PJ, DeMatteo RP, Fong Y, et al. Preoperative chemotherapy and the risk of hepatotoxicity and morbidity after liver resection for metastatic colorectal cancer: a single institution experience. J Am Coll Surg 2013; 216(1):41-9. 
58. Iorio A, Spencer FA, Falavigna M, Alba C, Lang E, Burnand B, et al. Use of GRADE for assessment of evidence about prognosis: rating confidence in estimates of event rates in broad categories of patients. BMJ 2015; 350:h870.

59. Huguet A, Hayden JA, Stinson J, McGrath PJ, Chambers CT, Tougas ME, et al. Judging the quality of evidence in reviews of prognostic factor research: adapting the GRADE framework. Syst Rev 2013; 2:71.

60. Stremitzer S, Stift J, Singh J, Starlinger P, Gruenberger B, Tamandl D, et al. Histological response, pattern of tumor destruction and clinical outcome after neoadjuvant chemotherapy including bevacizumab or cetuximab in patients undergoing liver resection for colorectal liver metastases. Eur J Surg Oncol 2015; 41(7):868-74.

61. Ribero D, Wang H, Donadon M, Zorzi D, Thomas MB, Eng C, et al. Bevacizumab improves pathologic response and protects against hepatic injury in patients treated with oxaliplatin-based chemotherapy for colorectal liver metastases. Cancer 2007; 110(12):2761-7.

62. Economopoulou P, Kotsakis A, Kapiris I, Kentepozidis N. Cancer therapy and cardiovascular risk: focus on bevacizumab. Cancer Manag Res 2015; 7:133-43.

63. Varga ZV, Ferdinandy P, Liaudet L, Pacher P. Drug-induced mitochondrial dysfunction and cardiotoxicity. Am J Physiol Heart Circ Physiol 2015; 309(9):H1453-67.

64. Aussilhou B, Dokmak S, Faivre S, Paradis V, Vilgrain V, Belghiti J. Preoperative liver hypertrophy induced by portal flow occlusion before major hepatic resection for colorectal metastases can be impaired by bevacizumab. Ann Surg Oncol 2009; 16(6):1553-9.

65. Gruenberger B, Tamandl D, Schueller J, Scheithauer W, Zielinski C, Herbst F, et al. Bevacizumab, capecitabine, and oxaliplatin as neoadjuvant therapy for patients with potentially curable metastatic colorectal cancer. J Clin Oncol 2008; 26(11):1830-5.

66. Dejong $\mathrm{CH}$. Vascular reconstruction combined with liver resection for malignant tumours. Br J Surg 2013; 100(13):1776.

67. Reddy SK, Pawlik TM, Zorzi D, Gleisner AL, Ribero D, Assumpcao L, et al. Simultaneous resections of colorectal cancer and synchronous liver metastases: a multi-institutional analysis. Ann Surg Oncol 2007; 14(12):3481-91.

68. van Lienden KP, van den Esschert JW, de Graaf W, Bipat S, Lameris JS, van Gulik TM, et al. Portal vein embolization before liver resection: a systematic review. Cardiovasc Intervent Radiol 2013; 36(1):25-34.

69. Vauthey JN, Chaoui A, Do KA, Bilimoria MM, Fenstermacher MJ, Charnsangavej C, et al. Standardized measurement of the future liver remnant prior to extended liver resection: methodology and clinical associations. Surgery 2000; 127(5):512-9.

70. Riley RD, Lambert PC, Abo-Zaid G. Meta-analysis of individual participant data: rationale, conduct, and reporting. BMJ 2010; 340:c221 


\section{Appendix 2.1. Full electronic search}

The full electronic search strategy that was performed for the present study in multiple international databases

Searches were limited to retrieve results from 01.01.2004-09.06.2015

- Medline (OvidSP): 2004-2015/05/WK5

- Medline In-Process Citations \& Daily Update (OvidSP): up to 2015/06/08

- Embase (OvidSP): 2004-2015/06/08

- Cochrane Central Register of Controlled Trials (CENTRAL) (Wiley) 2004-Issue 5, 2015/05

- LILACS (Latin American and Caribbean Health Sciences Literature) (Internet) http://lilacs.bvs.alud.org/en/: 2004-2015/06/03

- CINAHL (Cumulative Index to Nursing and Allied Health Literature) (EBSCO): 20042015/06/05

\section{Strategies}

\section{Medline (OvidSP): 2004-2015/05/WK5}

\section{Searched 9.6.15}

1 Hepatic Veno-Occlusive Disease/ (1105)

2 ((venocclusi\$ or venoocclusi\$ or veno occlusi\$) adj2 (liver or livers or hepato\$ or hepatic\$)).ti,ab,ot. (667)

3 Stuart bras syndrome.ti,ab,ot. (3)

4 ((SOS or VOD or HVOD) adj5 (liver or livers or hepatic\$ or hepato\$)).ti,ab,ot. (460)

5 (sinusoid\$ adj2 (obstruct\$ or dilat\$ or injur\$ or damage\$)).ti,ab,ot. (898)

6 or/1-5 (2164)

7 exp animals/ not (exp animals/ and humans/) (4056152)

86 not 7 (1785)

9 limit 8 to $y r=" 2004$-Current" (742) 


\section{Medline In-Process Citations (OvidSP): up to 2015/06/08}

\section{Medline Daily Update (OvidSP): up to 2015/06/08}

\section{Searched 9.6.15}

1 Hepatic Veno-Occlusive Disease/ (0)

2 ((venocclusi\$ or venoocclusi\$ or veno occlusi\$) adj2 (liver or livers or hepato\$ or hepatic\$)).ti,ab,ot. (26)

3 Stuart bras syndrome.ti,ab,ot. (0)

4 ((SOS or VOD or HVOD) adj5 (liver or livers or hepatic\$ or hepato\$)).ti,ab,ot. (22)

5 (sinusoid\$ adj2 (obstruct\$ or dilat\$ or injur\$ or damage\$)).ti,ab,ot. (91)

6 or/1-5 (112)

7 exp animals/ not (exp animals/ and humans/) (2153)

86 not $7(112)$

9 limit 8 to $y r=" 2004$-Current" (110)

\section{Embase (OvidSP): 2004-2015/6/8}

\section{Searched 9.6.15}

1 liver venoocclusive disease/ (1279)

2 ((venocclusi\$ or venoocclusi\$ or veno occlusi\$) adj2 (liver or livers or hepato\$ or hepatic\$)).ti,ab,ot. (887)

3 Stuart bras syndrome.ti,ab,ot. (3)

4 ((SOS or VOD or HVOD) adj5 (liver or livers or hepatic\$ or hepato\$)).ti,ab,ot. (769)

5 (sinusoid\$ adj2 (obstruct\$ or dilat\$ or injur\$ or damage\$)).ti,ab,ot. (1514)

6 or/1-5 (3329)

7 animal/ (1665207)

8 animal experiment/ (1854326)

9 (rat or rats or mouse or mice or murine or rodent or rodents or hamster or hamsters or pig or pigs or porcine or rabbit or rabbits or animal or animals or dogs or dog or cats or cow or bovine or sheep or ovine or monkey or monkeys). ti,ab,ot,hw. (5941840)

10 or/7-9 (5941840)

11 exp human/ (16002764)

12 human experiment/ (337714)

13 or/11-12 (16004207)

1410 not (10 and 13) (4709608)

156 not 14 (2805)

16 limit 15 to $y r=" 2004$-Current" (1833) 
Cochrane Central Register of Controlled Trials (CENTRAL) (Wiley): Issue 5/ May 2015 Searched 11.3.15

\#1 MeSH descriptor: [Hepatic Veno-Occlusive Disease] explode all trees 40

\#2 ((venocclusi* or venoocclusi* or veno occlusi*) near/2 (liver or livers or hepato* or hepatic*)):ti,ab,kw 62

\#3 Stuart bras syndrome:ti,ab 1

\#4 ((SOS or VOD or HVOD) near/5 (liver or livers or hepatic* or hepato*)):ti,ab,kw 36

\#5 (sinusoid* near/2 (obstruct* or dilat* or injur* or damage*)) .ti,ab,ot. 0

\#6 \#1 or \#2 or \#3 or \#4 or \#5 Publication Year from 2004 to 201540

CENTRAL results $=30$

Literature in the Health Sciences in Latin America and the Caribbean (LILACS) (Internet): 2004-2015/06/03

Searched 9.6.15

Advanced search

Limited to 2004-2015

Limited to Humans only

Limited to: LILACS

(tw:((mh: c06.552.360 OR mh: c14.907.460 OR mh: hepatic veno-occlusive disease OR sinusoidal obstruction OR sinusoidal injury OR "stuart bras syndrome" OR "Enfermedad Veno-Oclusiva Hepática" OR "Hepatopatia Veno-Oclusiva")) OR (tw:(((sos OR vod OR venocclusi* OR venoocclusi* OR veno-occlusi*) AND (liver OR livers OR hepatic* OR hepato*))))) OR (tw:((sinusoid* AND (obstruct* OR dilat* OR injur* OR damage*)))) $\mathrm{N}=26$

CINAHL (Cumulative Index to Nursing and Allied Health Literature) (EBSCO): 20042015/06/05

\section{Searched 9.6.15}

S1 MH Hepatic Veno-Occlusive Disease Search modes 0

S2 TI ((venocclusi* or venoocclusi* or veno occlusi*) N2 (liver or livers or hepato* or hepatic*)) 7

S3 AB ((venocclusi* or venoocclusi* or veno occlusi*) N2 (liver or livers or hepato* or hepatic*)) 13

S4 TI Stuart bras syndrome 0

S5 AB Stuart bras syndrome 0

S6 TI ((SOS or VOD or HVOD) N5 (liver or livers or hepatic* or hepato*)) 0 
S7 AB ((SOS or VOD or HVOD) N5 (liver or livers or hepatic* or hepato*)) 6

S8 TI (sinusoid* N2 (obstruct* or dilat* or injur* or damage*)) 14

S9 AB (sinusoid* N2 (obstruct* or dilat* or injur* or damage*)) 19

S10 S1 OR S2 OR S3 OR S4 OR S5 OR S6 OR S7 OR S8 OR S9 44

S11 S1 OR S2 OR S3 OR S4 OR S5 OR S6 OR S7 OR S8 OR S9 Limiters - Published Date: 20040101-2015123136 


\section{Appendix 2.2. Sub analyses of postoperative short-term outcomes SD grade 1-3 vs. SD grade 0}

Meta-analyses on the influence of sinusoidal dilatation (SD) grade 1-3 versus SD grade 0 on the complication rate after partial hepatectomy for colorectal liver metastases

Appendix 2.2a. Postoperative overall morbidity

Meta-analysis on the influence of sinusoidal dilatation (SD) grade 1-3 versus SD grade 0 on the morbidity rate due to any cause after partial hepatectomy for colorectal liver metastases

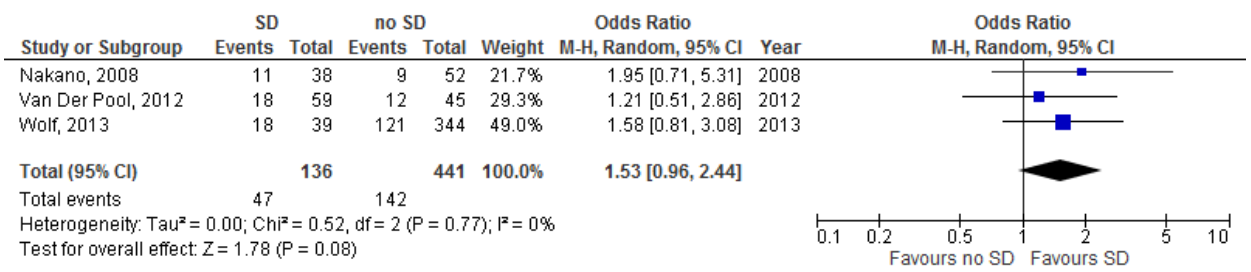

\section{Appendix 2b. Postoperative overall mortality}

Meta-analysis on the influence of sinusoidal dilatation (SD) grade 1-3 versus SD grade 0 on the mortality rate due to any cause after partial hepatectomy for colorectal liver metastases

SD no SD $\quad$ Odds Ratio

Study or Subgroup Events Total Events Total Weight M-H, Random, 95\% Cl Year

$\begin{array}{llllllll}\text { Nguyen-Khac, } 2013 & 0 & 24 & 2 & 26 & 48.3 \% & 0.20[0.01,4.38] & 2013\end{array}$

$\begin{array}{llllllll}\text { Wolf, } 2013 & 0 & 39 & 3 & 344 & 51.7 \% & 1.24[0.06,24.35] & 2013\end{array}$

$\begin{array}{lllll}\text { Total }(95 \% \mathrm{Cl}) & 63 & 370 & 100.0 \% & 0.51[0.06,4.38]\end{array}$

$\begin{array}{lcc}\text { Total events } & 0 & 5 \\ \text { Heterogeneity: } \operatorname{Tau}^{2}=0.00 ; & \mathrm{Ch}^{2}=0.71, \mathrm{df}=1(\mathrm{P}=0.40) ; \mathrm{I}^{2}=0 \%\end{array}$

Heterogeneity: Tau $^{2}=0.00 ; \mathrm{Chi}^{2}=0.71, \mathrm{df}=1(P=0.40) ; \mathrm{I}^{2}=0 \%$
Test for overall effect: $Z=0.61(P=0.54)$






\section{Appendix 2.2c. Postoperative liver-related morbidity}

Meta-analysis on the influence of sinusoidal dilatation (SD) grade 1-3 versus SD grade 0 on the liver-related morbidity rate after partial hepatectomy for colorectal liver metastases

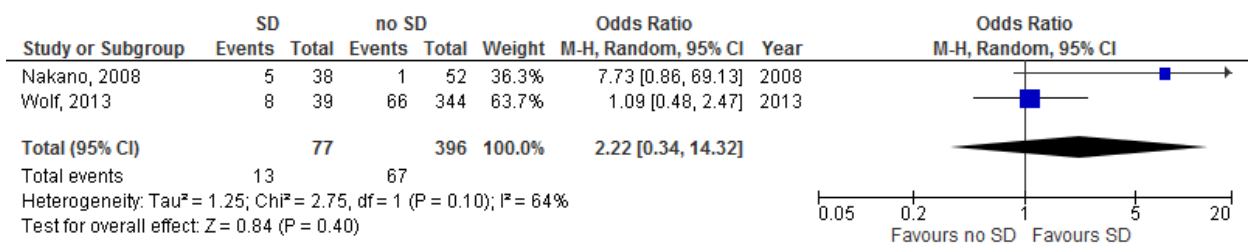

SD, sinusoidal dilatation; $\mathrm{M}-\mathrm{H}$, Mantel-Haenszel; Random, random effects model; $95 \% \mathrm{Cl}$, 95\% confidence interval 


\section{Appendix 2.3. Sub analyses of postoperative short-term outcomes SD grade 1-3 vs. SD grade 0 - major liver resections}

Meta-analyses on the influence of sinusoidal dilatation (SD) grade 1-3 versus SD grade 0 on the complication rate after major hepatectomy for colorectal liver metastases

Appendix 2.3a. Postoperative overall morbidity

Meta-analysis on the influence of sinusoidal dilatation (SD) grade 1-3 versus SD grade 0 on the morbidity rate due to any cause after major hepatectomy for colorectal liver metastases

\begin{tabular}{|c|c|c|c|c|c|c|c|c|c|c|}
\hline \multirow{2}{*}{ Study or Subgroup } & \multicolumn{2}{|c|}{ SD } & \multicolumn{2}{|c|}{ no SD } & \multirow{2}{*}{$\begin{array}{c}\text { Odds Ratio } \\
\text { M- } \mathrm{H}, \text { Random, } 95 \% \mathrm{Cl}\end{array}$} & \multirow{2}{*}{ Year } & \multirow{2}{*}{\multicolumn{4}{|c|}{$\begin{array}{c}\text { Odds Ratio } \\
\text { M-H, Random, } 95 \% \mathrm{Cl}\end{array}$}} \\
\hline & Events & Total & Events & Total & & & & & & \\
\hline Nakano, 2008 & 8 & 20 & 1 & 16 & $10.00[1.09,91.44]$ & 2008 & & & & \\
\hline Narita, 2012 & 22 & 44 & 36 & 57 & $0.58[0.26,1.30]$ & 2012 & & 1 & & \\
\hline & & & & & & & 0.01 & $\begin{array}{l}0.1 \\
\text { Favours no SD }\end{array}$ & $\begin{array}{r}10 \\
\text { Favours SD }\end{array}$ & 100 \\
\hline
\end{tabular}

Appendix 2.3b. Postoperative liver failure

Meta-analysis on the influence of sinusoidal dilatation (SD) grade 1-3 versus SD grade 0 on the liver failure rate after major hepatectomy for colorectal liver metastases

\begin{tabular}{|c|c|c|c|c|c|c|c|c|c|c|c|}
\hline \multirow[b]{2}{*}{ Study or Subgroup } & \multicolumn{2}{|l|}{ SD } & \multicolumn{2}{|c|}{ no SD } & \multicolumn{3}{|c|}{ Odds Ratio } & \multirow{2}{*}{\multicolumn{4}{|c|}{$\begin{array}{c}\text { Odds Ratio } \\
\text { M-H, Random, } 95 \% \mathrm{Cl}\end{array}$}} \\
\hline & Events & Total & Events & Total & Weight & M-H, Random, $95 \% \mathrm{Cl}$ & Year & & & & \\
\hline Nakano, 2008 & 2 & 20 & 0 & 16 & $11.2 \%$ & $4.46[0.20,99.79]$ & 2008 & & & & \\
\hline Narita, 2012 & 10 & 44 & 6 & 57 & $88.8 \%$ & $2.50[0.83,7.52]$ & 2012 & & & & \\
\hline Total $(95 \% \mathrm{Cl})$ & & 64 & & 73 & $100.0 \%$ & $2.67[0.94,7.53]$ & & & & & \\
\hline Total events & 12 & & 6 & & & & & & & & \\
\hline $\begin{array}{l}\text { Heterogeneity: Tau² } \\
\text { Test for overall effect }\end{array}$ & $\begin{array}{l}0.00 ; \mathrm{Ch} \\
\mathrm{Z}=1.85\end{array}$ & $\begin{array}{l}P=0.1 \\
P=0.0\end{array}$ & $\begin{array}{l}2, d f=1( \\
6)\end{array}$ & $P=0.7$ & $3) ;\left.\right|^{2}=0 \%$ & & & 0.01 & $\begin{array}{c}0.1 \\
\text { Favours no SD }\end{array}$ & $\begin{array}{r}10 \\
\text { Favours SD }\end{array}$ & 100 \\
\hline
\end{tabular}

SD, sinusoidal dilatation; $\mathrm{M}-\mathrm{H}$, Mantel-Haenszel; Random, random effects model; $95 \% \mathrm{Cl}, 95 \%$ confidence interval 












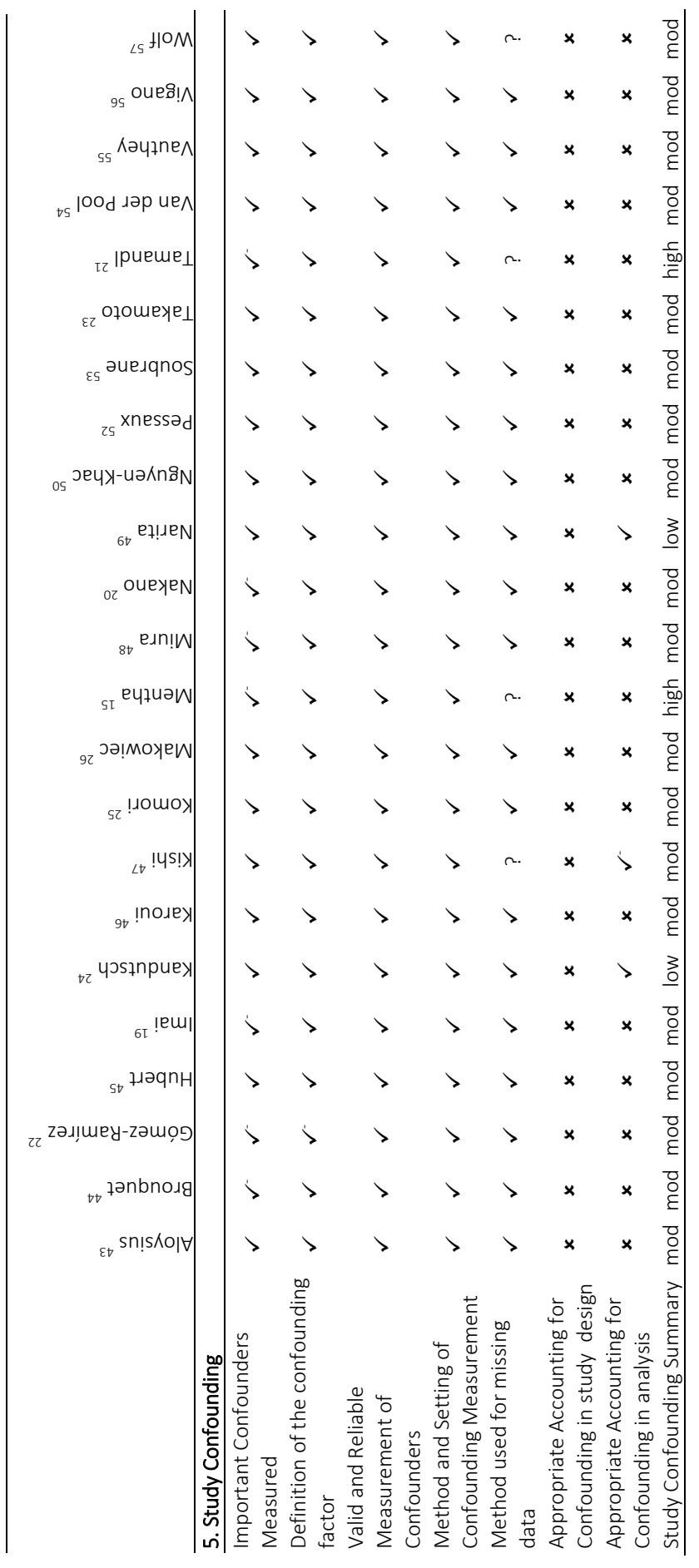









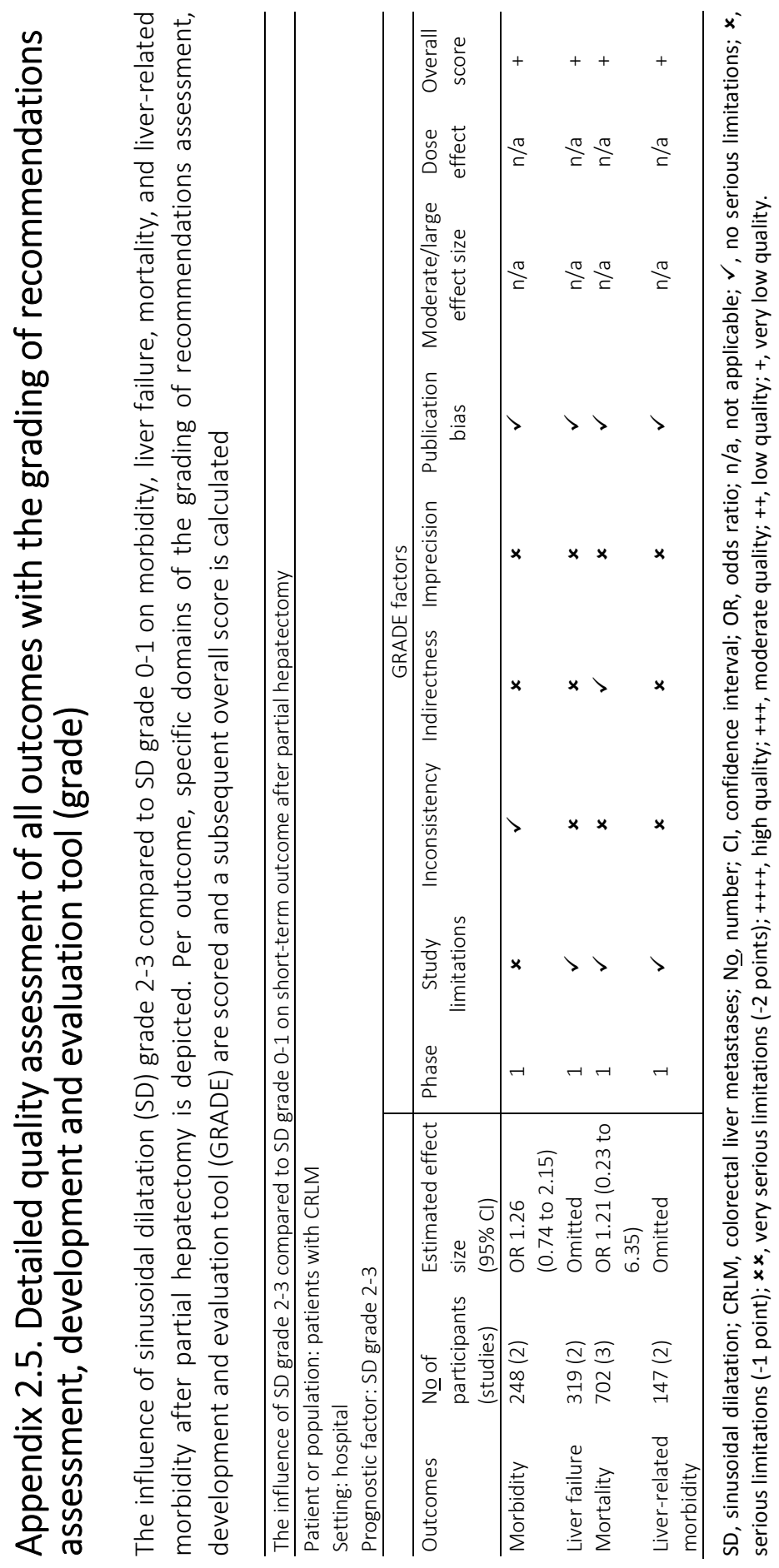




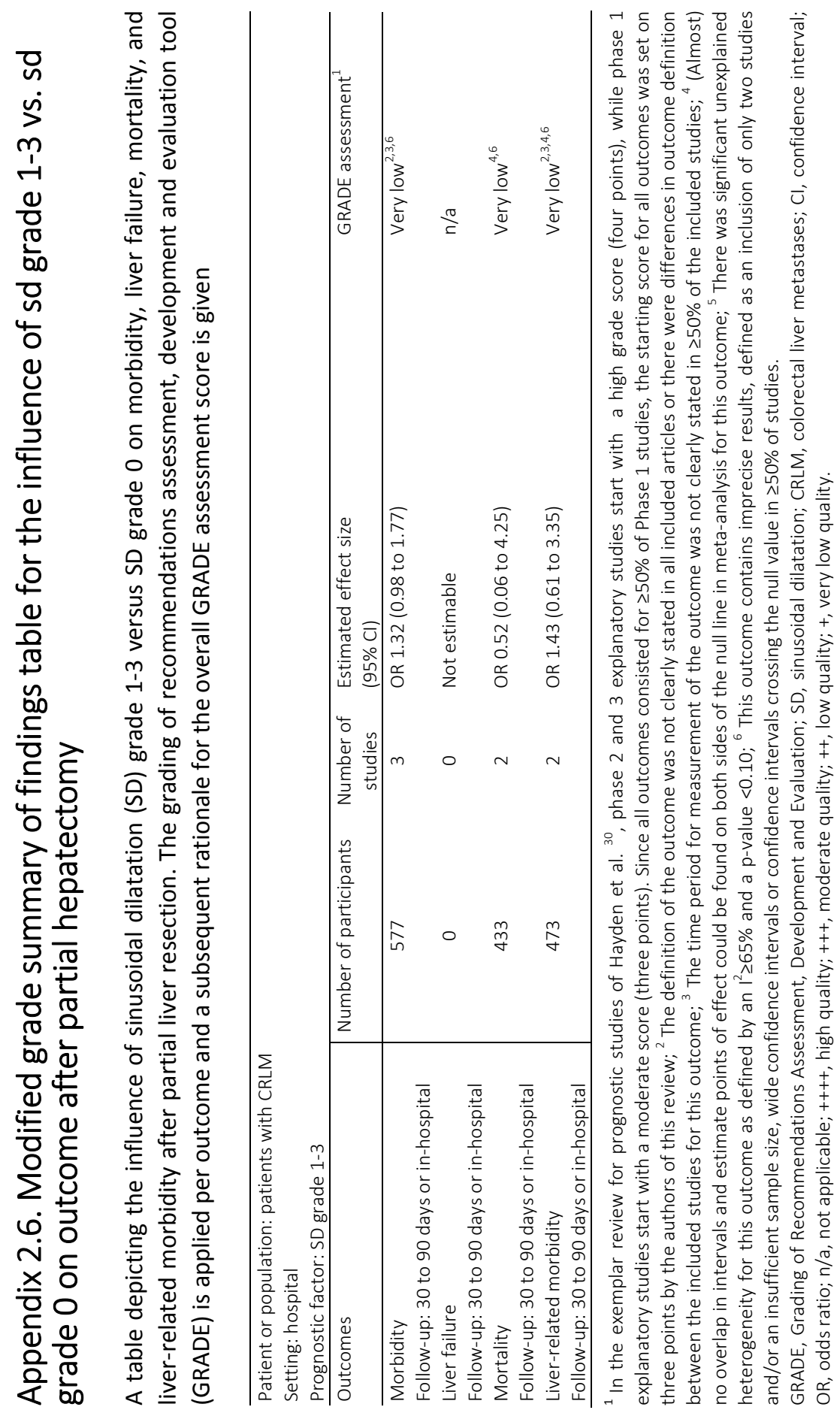




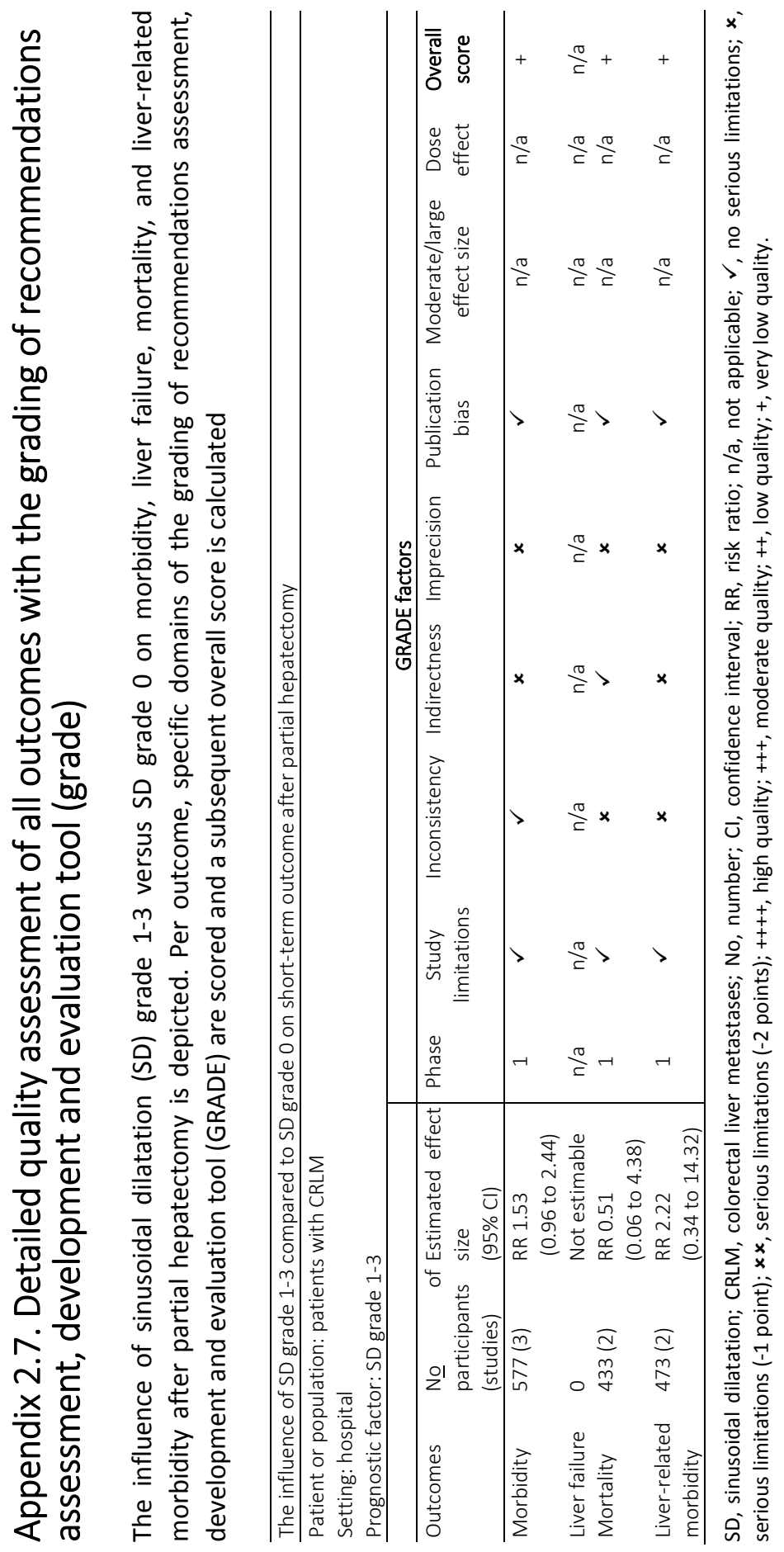




\section{Chapter}

\section{Systematic review of the influence of chemotherapy-}

associated liver injury on outcome after partial hepatectomy for colorectal liver metastases

Junfang Zhao*, Kim M.C. van Mierlo*, Joaquín Gómez-Ramírez, Haeryoung Kim,

Charles H.C. Pilgrim, Patrick Pessaux, Sander S. Rensen, Eric P. van der Stok,

Frank G. Schaap, Olivier Soubrane, Takeshi Takamoto, Luca Viganò, Bjorn Winkens, Cornelis H.C. Dejong, Steven W.M. Olde Damink; the Chemotherapy-associated Liver Injury (CALI) consortium *Both authors contributed equally to this study Br J Surg. 2017;104(8):990-1002 


\section{Abstract}

\section{Background}

The impact of chemotherapy-associated liver injury (CALI) on postoperative outcome in patients undergoing partial hepatectomy for colorectal liver metastases (CRLM) remains controversial. The objective of this study was to clarify the effect of CALI (sinusoidal dilatation (SD), steatosis, and steatohepatitis) on postoperative morbidity and mortality by investigating a large dataset from multiple international centres.

\section{Methods}

Pubmed and Embase were searched for studies published between 1 January 2004 and 31 December 2013 with keywords: "chemotherapy", "liver resection", "outcome", and "colorectal metastases" to identify potential collaborating centres. Univariable and multivariable analyses were performed using binary logistic regression models, with results presented as odds ratio (ORs) with 95\% confidence intervals (Cls).

\section{Results}

A consolidated database comprising 788 patients who underwent hepatectomy for CRLM in eight centres was obtained. In multivariable analyses, severe SD was associated with increased major morbidity (Dindo-Clavien grade III-V; OR 1.73, 95\% Cl 1.02-2.95, $\mathrm{p}=0.043)$. Severe steatosis was associated with decreased liver surgery-specific complications (OR 0.52, 95\% Cl 0.27-1.00, p=0.049), whereas steatohepatitis was linked to an increase in these complications (OR 2.08, 95\% Cl 1.18-3.66, $\mathrm{p}=0.012$ ). Subgroup analysis showed that lobular inflammation was the sole component associated with increased overall morbidity ( $\mathrm{OR} 2.22,95 \% \mathrm{Cl} 1.48-3.34, \mathrm{p}=0.001)$ and liver surgeryspecific complications (OR 3.35, 95\% Cl 2.11-5.32, $\mathrm{p}<0.001$ ). Finally, oxaliplatin treatment was linked to severe SD (OR 2.74, 95\% Cl 1.67-4.49, $\mathrm{p}<0.001)$.

\section{Conclusions}

An increase in postoperative major morbidity and liver surgery-specific complications was observed after partial hepatectomy in patients with severe SD and steatohepatitis. Moreover, postoperative liver failure occurred more often in patients with severe SD. 


\section{Introduction}

Colorectal cancer (CRC) is the third most common cancer worldwide, affecting over 1.3 million patients annually. ${ }^{1}$ Approximately $50 \%$ of these patients develop colorectal liver metastases (CRLM). ${ }^{2,3}$ Although liver resection provides the best prospect of cure, only $10-30 \%$ of patients with liver metastases are eligible for hepatic surgery., ${ }^{3,4}$ For patients with tumours deemed unresectable, neoadjuvant systemic chemotherapy can prolong survival, and allow future hepatic resection. ${ }^{2,5}$

For decades, 5-fluorouracil (5-FU) was the sole option for treating CRLM. This has changed markedly in the new millennium; with the approval of irinotecan, oxaliplatin, and humanized monoclonal antibodies, approximately $15 \%$ of patients with initially unresectable tumours became eligible for liver resection., ${ }^{6,7}$ Unfortunately, administration of irinotecan- and/or oxaliplatin-based chemotherapeutic agents has been associated with a harmful side-effect in the form of liver injury.

Chemotherapy-associated liver injury (CALI) is often reported in patients with CRLM, and appears to be regimen specific. For instance, oxaliplatin treatment is associated with sinusoidal obstruction syndrome (SOS), ${ }^{10}$ and linked to increased occurrence of nodular regenerative hyperplasia (NRH). ${ }^{11}$ Co-administration of bevacizumab with oxaliplatin, however, has been reported to be associated with a decrease in both the incidence and severity of SOS and $\mathrm{NRH}^{10-12}$ Irinotecan-based regimens appear to be related to the development of steatohepatitis. ${ }^{8,13,14}$ Importantly, because patients commonly receive several chemotherapeutic agents to offer optimal benefit in downsizing tumours, it is difficult to identify the specific agents responsible for injury to the hepatic parenchyma.

Certain studies, ${ }^{9,13,15-18}$ have claimed a negative correlation between CALI and postoperative outcome (postoperative morbidity, mortality), but others ${ }^{19-23}$ could not reproduce this. Therefore, it remains unclear whether CALI influences postoperative morbidity and mortality. The aim of the present study was to explore whether sinusoidal dilatation (SD), steatosis, and steatohepatitis are associated with increased morbidity and mortality rates after partial hepatectomy by performing a meta-analysis of individual participant data based on a systematic literature review. Additionally, factors associated with the occurrence of CALI were identified. 


\section{Methods}

An extensive protocol, written before the start of this study, can be found in Appendix 3.1. PRISMA and Moose guidelines were followed when conducting and reporting this review. ${ }^{24,25}$

\section{Search strategy for identification of studies}

Systematic searches were performed in MEDLINE (PubMed) and Embase for studies published between 1 January 2004 and 31 December 2013 using a search matrix including the following four categories: liver resection, chemotherapy, tumour type, and outcome. For the purpose of performing a more comprehensive search, the type of liver injury was not included in the searching matrix. The full search strategy is shown in the supplemental data (Appendix 3.1). The first publication date was fixed at 2004 because the widely used criteria for scoring SD, steatosis, and steatohepatitis were developed in $2004^{26}$ and $2005 .^{27}$ No language filter was applied.

\section{Inclusion criteria}

Studies meeting the following criteria were considered eligible for inclusion: adult patients (aged over 18 years), who underwent liver resection for CRLM, with description of postoperative short-term overall morbidity, liver surgery-specific complications, postoperative liver failure, or overall mortality (within 90 days or in-hospital) after liver resection, and with pathological assessment of non-tumorous liver specimens for SD, steatosis, and/or steatohepatitis (Appendix 3.1). Studies with patients who received preoperative hepatic arterial infusion of chemotherapy were excluded, as were case reports, comments, those with published abstracts only, editorials and reviews.

\section{Study identification and data collection}

Duplicates were removed. Two authors independently screened all titles and abstracts and excluded those not pertinent to the review. Any discrepancy was solved by consensus. The remaining articles were included for full-text revision and assessed independently for eligibility by the two authors. Reference lists of full-text reviewed articles were checked manually for additional potential citations, along with exploration of a personal library that was established during previous research on this topic. ${ }^{28,29}$ 


\section{Definitions}

SD was graded according to Rubbia-Brandt and colleagues, ${ }^{26}$ with grade 2-3 (severe SD) considered clinically relevant since it reflects rupture of sinusoidal wall integrity. SD is one of the most important histological features of SOS, and its severity is generally accepted to correspond to the severity of SOS. Steatosis and steatohepatitis were graded according to Kleiner et al. ${ }^{27}$ Severe steatosis was defined as more than $33 \%$ of parenchyma affected by steatosis. ${ }^{27}$ A non-alcoholic steatohepatitis activity score (NAS) of 4 or more was considered as steatohepatitis to provide a working cut-off value conforming with the literature. ${ }^{30} \mathrm{CALI}$ was defined as any occurrence of the severe SD, severe steatosis, or steatohepatitis. Co-morbidity was defined as any disease affecting the patient apart from colorectal liver metastases (diabetes mellitus, pulmonary, renal, cardiovascular and other diseases). Overall morbidity was defined as any complication occurring within 90 days after surgery or during the hospital stay, and graded according to the classification of Dindo and colleagues. ${ }^{31}$ Major morbidity was defined as DindoClavien grade III (requiring invasive intervention) or higher. The concept of a liver surgery-specific complication was in correspondence to the liver surgery-specific composite endpoint developed in 2011, and included one or more of the following events: ascites, postoperative liver failure, bile leakage, intra-abdominal abscess, intraabdominal haemorrhage, and operative mortality. ${ }^{32}$ Postoperative liver failure was defined as the concurrent presence of a prothrombin time below $50 \%$ and a serum bilirubin greater than $50 \mu \mathrm{M} / \mathrm{L}$ (the $50-50$ criteria) on or after postoperative day $5 .{ }^{33}$ Postoperative mortality included death from any cause within 90 days after surgery or during hospital admission. Major hepatectomy was defined as resection of three or more Couinaud liver segments. ${ }^{34}$

\section{Risk-of-bias assessment}

The risk of bias of the final included studies was assessed independently by two blinded researchers using the quality in prognosis studies (QUIPS) tool. ${ }^{35,36}$ Discrepancies were discussed by the two authors and consensus was reached. The QUIPS tool includes six bias domains: participation, attrition, prognostic factor measurement, confounding measurement and account, outcome measurement, and analysis and reporting. The results of each of the six domains were taken together to calculate an overall low, moderate, or high risk of bias, as follows: overall low risk of bias, two or fewer domains rated as moderate risk and the remaining domains as low risk; overall moderate risk, at least three domains rated as moderate risk and the remaining domains as low risk; and high overall risk, one or more domain rated as high risk, independent of the rating of the remaining domains. 


\section{Data handling and statistical methods}

Corresponding authors from studies that fulfilled the inclusion criteria were contacted by email for collaboration and sharing of coded data (by numbering) for the published cohort. Each author was asked to sign a specific data transfer agreement form, which assured careful handling of the data. Coded data were arranged in a preconstructed Excel $^{\mathrm{TM}}$ file (Microsoft, Redmond, Washington, USA) and subsequently imported into SPSS $^{\circledR}$ for Windows ${ }^{\circledR}$ version 20.0 (IBM, Armonk, New York, USA).

Patient characteristics were compared using the Student's $t$ test for numerical variables. For categorical variables, the Pearson Chi-square test with continuity correction was applied, or Fisher's exact test when any of the expected values was smaller than five. The influence of preoperative chemotherapeutic agents on liver injury, and subsequently the effect of liver injury on short-term postoperative outcome were analysed applying one-step binary logistic regression models. Individual participant data from all studies were pooled and modelled simultaneously. This approach was considered more optimal because each study showed relatively few events per outcome and small sample sizes, and the one-step approach for pooled data allowed the exact binomial distribution to be used and did not require continuity corrections when there were no events. ${ }^{37,38}$ For missing values, multiple imputations were performed, assuming that data were missing at random. The number of imputations was determined by the maximum percentage of missing data in the data set. In this study, 30 imputations were performed, as the maximum amount of missing data was 26\% (minor/major morbidity). Variables in multiple imputations are listed in Appendix 3.2. A complete-case sensitive analysis was also conducted. Clustering of patients from different studies was integrated as a separate co-variable ('database source') and included in binary logistic regression models in every analysis. ${ }^{38}$ A single variable together with database source created the univariable model. All variables with $p \leq 0.200$ in univariable analysis were included in the multivariable analysis. In addition, database source and variables known to be related to the outcome (either well-described in literature or based on careful discussion and consensus between the authors) were forced into the multivariable model.

A subgroup analysis including only patients who received oxaliplatin-based treatment was performed to investigate the impact of bevacizumab on the occurrence of severe SD. At a later stage of the study, data to investigate the influence of separate NASsubcategories (steatosis, lobular inflammation and hepatocellular ballooning) on postoperative outcome were requested. A subgroup analysis including cohorts with available data on NAS-subcategories was undertaken. The methods of analysis of these data were identical to those described above. Unadjusted and adjusted odds ratios (ORs) 
with $95 \%$ confidence intervals $(95 \% \mathrm{Cls}$ ) were calculated. $\mathrm{p} \leq 0.050$ was considered significant for all analyses.

\section{Results}

The search strategy resulted in 1191 unique hits (Figure 3.1). In total, 1093 articles were excluded on the base of title or abstract, and the remaining 98 articles were subjected to full-text evaluation. Thirty-two studies met the inclusion criteria and the 30 corresponding authors were contacted by e-mail. Nineteen authors responded, of whom eight agreed to share their raw data. Other authors could not be contacted (Appendix 3.3). Potential publication bias was excluded by testing for asymmetry in a funnel plot. The eight included studies ${ }^{16,17,39-44}$ were assessed for risk of bias using the QUIPS tool. Overall ratings are shown in Table 3.1. Six articles showed an overall low risk of bias and one study each showed a moderate or high risk. Detailed scorings can be found in Appendix 3.4. Because the individual participant data from all studies were pooled and modelled simultaneously, an overall rating was also calculated without taking the study confounding and statistical analysis domains into account; all studies then showed a low risk of bias based on the remaining four domains.

Characteristics of the included studies are summarized in Table 3.1. The consolidated cohort consisted of 816 patients (Figure 3.1). Twenty-eight patients were excluded because they underwent surgery for indications other than CRLM, leaving 788 patients for analysis. Patient characteristics, surgical details, and postoperative outcomes of the consolidated cohort are listed in Table 3.2. There were 453 men (57.5\%) and 335 women (42.5\%) with a median age of 61 (range 25-86) years. SD was severe in 183 patients (24.1\%), steatosis was severe in 117 (15.6\%), and steatohepatitis in 100 (14.5\%). Of the 525 patients who received 5-FU, 396 (75.4\%) had simultaneous treatment with oxaliplatin and 135 (25.7\%) with irinotecan. Of the 136 patients who received capecitabine, 119 (87.5\%) had simultaneous treatment with oxaliplatin. Cetuximab and bevacizumab were most frequently administered together with oxaliplatin. All 61 patients who received cetuximab, and 138 of 164 (84.1\%) patients who received bevacizumab, were co-treated with oxaliplatin. Of 635 patients with NAS subcategory data, $96(15.1 \%)$ had a NAS of 4 or more. Only three patients (3.1\%) with a NAS of 4 or higher did not show lobular inflammation. The relationship between NAS and lobular inflammation is summarized in Appendix 3.5. 
Database retrieved hits $(\mathrm{n}=1473)$

Pubmed: 997

Embase: $\quad 476$

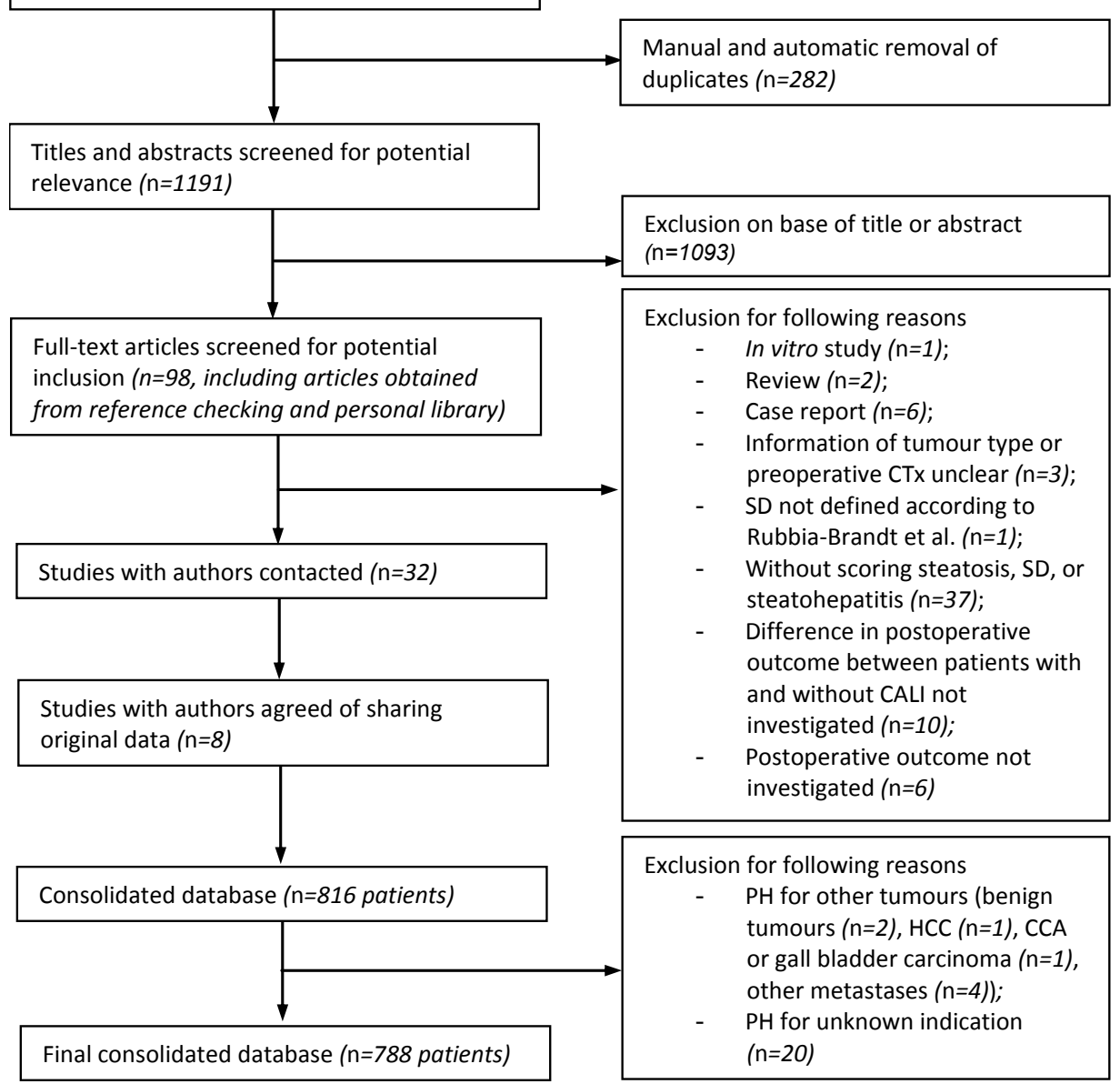

Figure 3.1 Selection process of included articles.

CTx, chemotherapy; SD, sinusoidal dilatation; CALI, chemotherapy-associated liver injury; PH, partial hepatectomy; HCC, hepatocellular carcinoma; CCA, cholangiocarcinoma. 


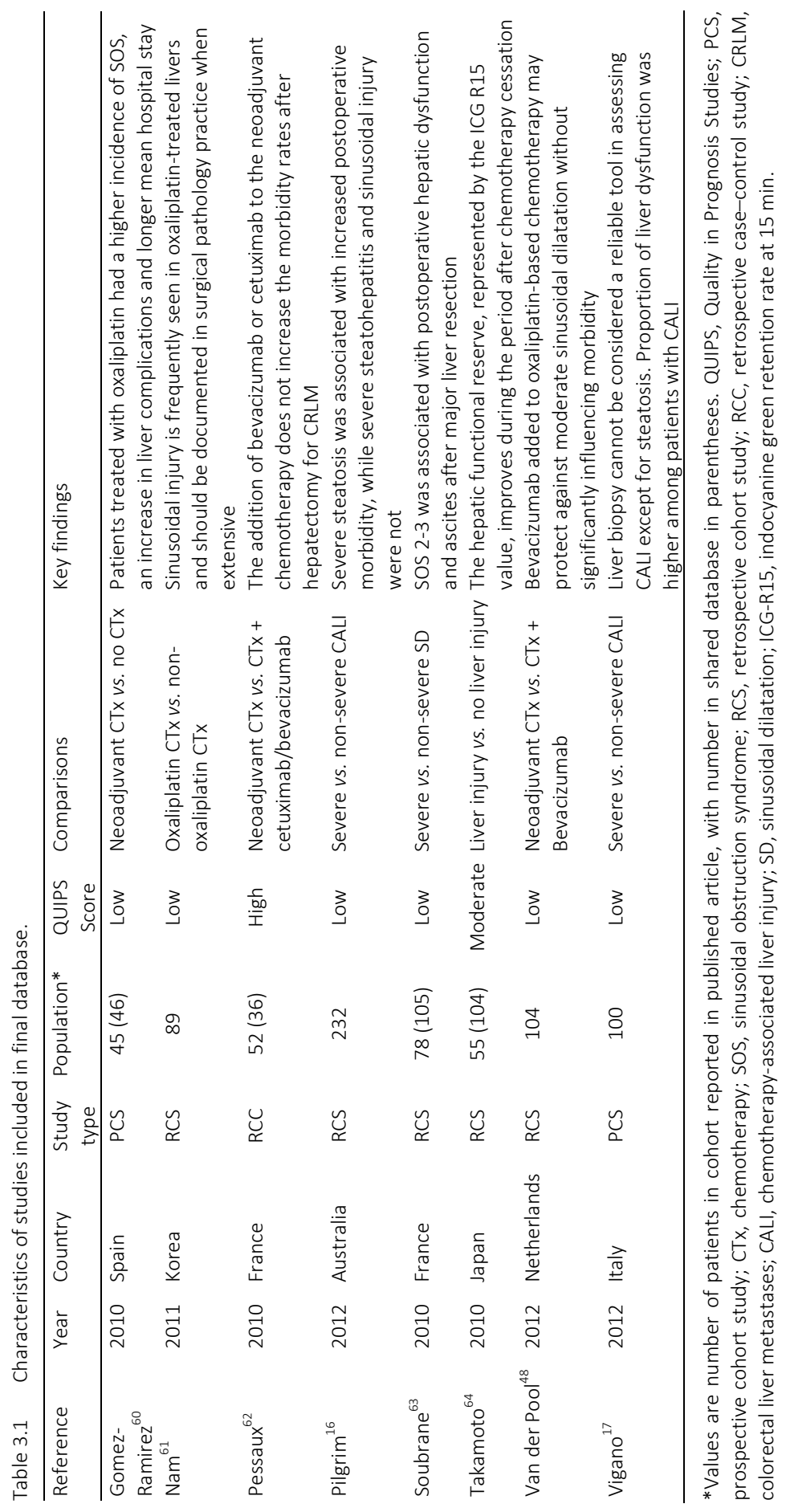




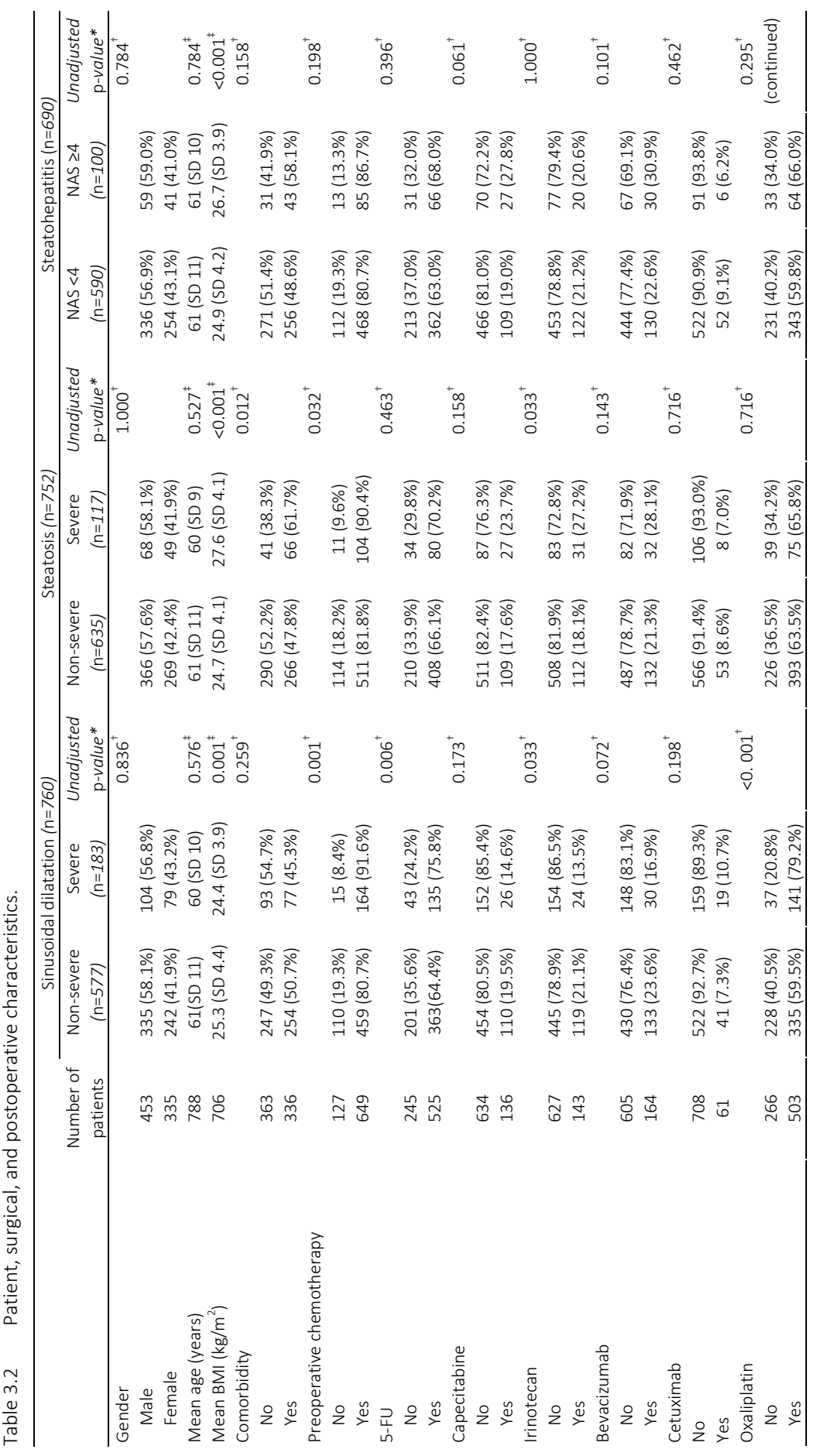




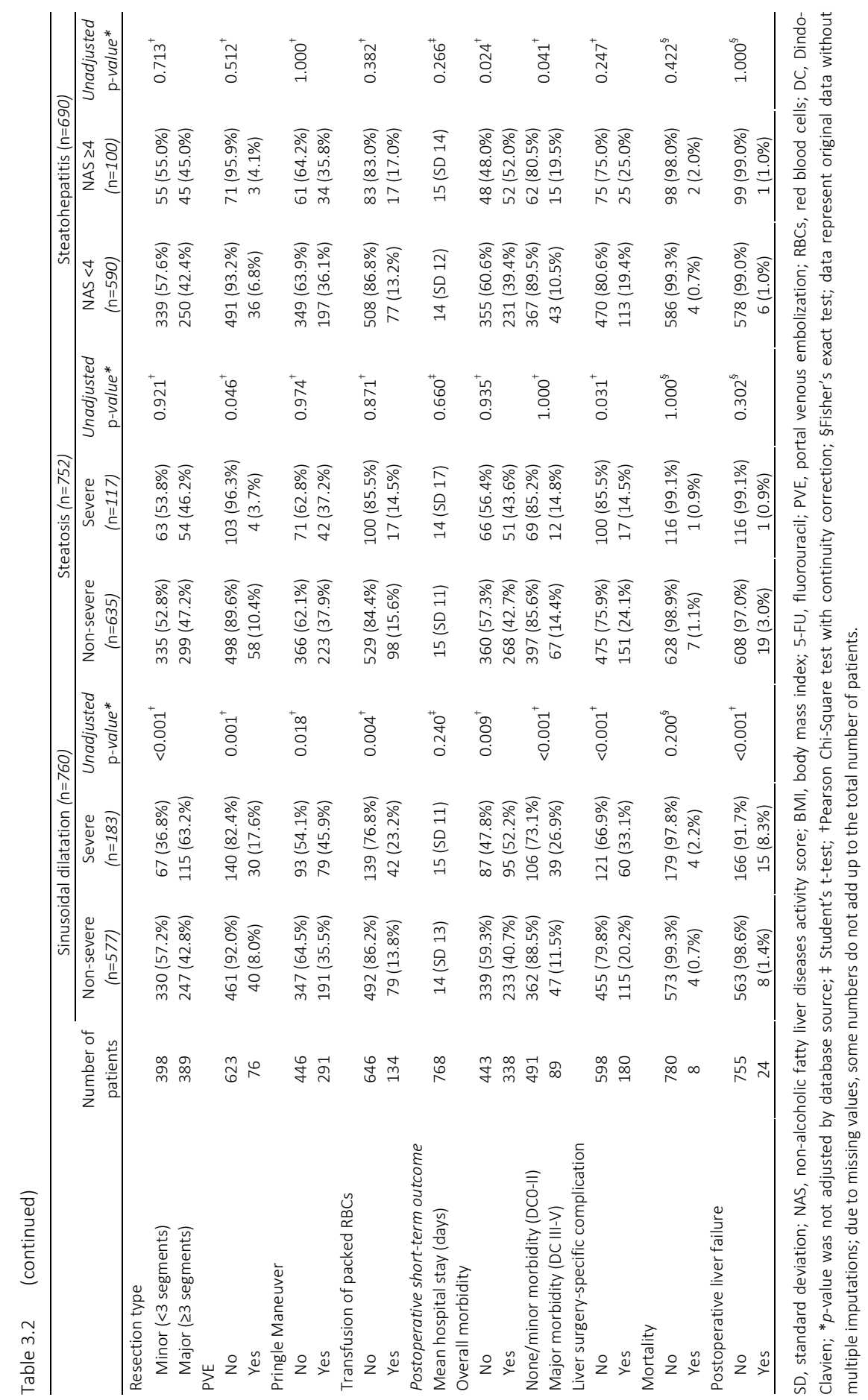


Sensitivity analysis showed similar results between complete case analysis and that with multiple imputations (detailed information available on request). The influence of severe $\mathrm{SD}$, severe steatosis, steatohepatitis, and other potential factors related to short-term overall morbidity, liver surgery-specific complications, and major morbidity is shown in Figure 3.2 and Appendix 3.6. Severe SD was significantly associated with increased major morbidity only (OR 1.73, 95\% Cl 1.02-2.95, $\mathrm{p}=0.043$ ). Severe steatosis was not significantly associated with the occurrence of postoperative overall or major morbidity, but was related to a decreased occurrence of liver surgery-specific complications (OR $0.52,95 \% \mathrm{Cl} 0.27-1.00, \mathrm{p}=0.049)$. In contrast, patients with steatohepatitis showed a significantly increased rate of postoperative liver surgery-specific complications (OR 2.08, 95\% Cl 1.18-3.66, $\mathrm{p}=0.012$ ), and a trend towards increased overall morbidity (OR $1.58,95 \% \mathrm{Cl} 0.99-2.52, p=0.057)$.

Overall morbidity

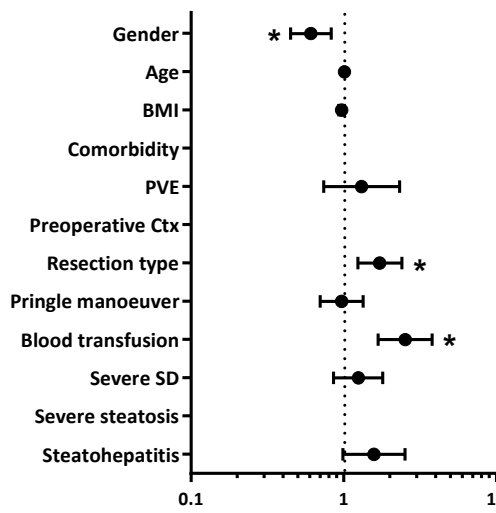

Liver surgery-specific complications

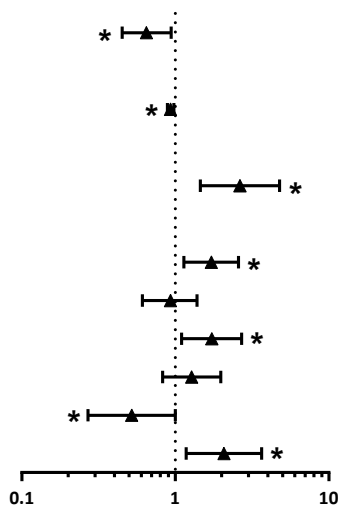

Major morbidity

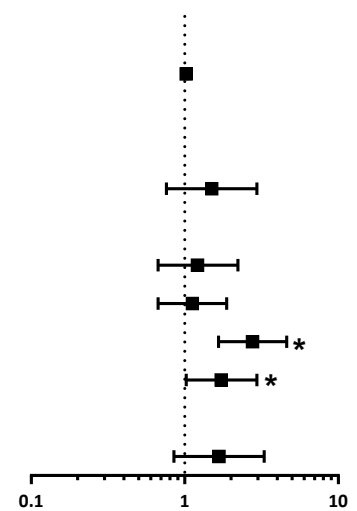

Figure 3.2 Multivariable analysis of influence of liver injury on short-term postoperative outcomes. Data are depicted as OR (symbol) with 95\% confidence intervals (bars) based on results of multivariable analysis; BMI, body mass index; PVE, portal venous embolization; Ctx, chemotherapy; SD, sinusoidal dilatation; asterisks indicate $p$-value $\leq 0.05$.

Table 3.3 summarizes the effects of several factors associated with postoperative liver failure and mortality. Because of the small number of events, multivariable logistic regression was not performed. Postoperative liver failure occurred in 24 of 779 patients (3.1\%) in the study cohort (Table 3.2), seven women and 17 men, with a median age of 61 (range 48-75) years. All patients with liver failure had undergone major hepatectomy. The only factors strongly associated with increased liver failure in univariable analysis 
were severe SD (OR 3.06, 95\% Cl 1.18-7.92, $\mathrm{p}=0.021)$ and perioperative blood transfusion (OR 4.47, 95\% Cl 1.69-11.82, $\mathrm{p}=0.003$ ).

Table 3.3 Univariable analyses of factors influencing short-term postoperative outcome.

\begin{tabular}{lcccc}
\hline & $\begin{array}{c}\text { Postoperative liver } \\
\text { failure }(n=24)\end{array}$ & & $\begin{array}{c}\text { Mortality } \\
(n=8)\end{array}$ & OR [95\% Cl] \\
\cline { 2 - 5 } Variable & OR [95\% Cl] & p-value & ORe \\
\hline Gender (female) & $0.65[0.25-1.68]$ & 0.376 & $0.50[0.10-2.25]$ & 0.408 \\
Age (years) & $1.00[0.96-1.04]$ & 0.937 & $1.03[0.96-1.05]$ & 0.402 \\
BMI $\left(\mathrm{kg} / \mathrm{m}^{2}\right)$ & $0.90[0.80-1.01]$ & 0.070 & $0.96[0.80-1.14]$ & 0.630 \\
Comorbidity & $1.28[0.53-3.13]$ & 0.583 & $7.84[0.92-66.65]$ & 0.059 \\
PVE & $2.62[0.95-7.26]$ & 0.064 & $1.09[0.13-9.09]$ & 0.938 \\
Preoperative chemotherapy & $2.41[0.30-19.32]$ & 0.409 & $1.51[0.52-4.42]$ & 0.702 \\
Resection type (major) & $\mathrm{n} / \mathrm{a}^{+}$ & $\mathrm{n} / \mathrm{a}^{+}$ & $6.56[0.77-55.90]$ & 0.085 \\
Pringle manoeuver & $1.45[0.57-3.72]$ & 0.437 & $1.91[0.43-8.54]$ & 0.399 \\
Blood transfusion & $4.47[1.69-11.82]$ & 0.003 & $14.00[2.74-71.51]$ & 0.002 \\
Severe SD & $3.06[1.18-7.92]$ & 0.021 & $2.79[0.68-11.44]$ & 0.155 \\
Severe steatosis & $0.47[0.06-3.77]$ & 0.473 & $0.86[0.10-7.09]$ & 0.887 \\
Steatohepatitis & $1.90[0.31-22.83]$ & 0.492 & $2.71[0.57-12.87]$ & 0.210 \\
\hline
\end{tabular}

BMI, body mass index; PVE, portal venous embolization; SD, sinusoidal dilatation; OR, odds ratio; $95 \% \mathrm{Cl}, 95 \%$ confidence intervals; $\mathrm{n} / \mathrm{a}$, not applicable; Multiple imputations were used for univariable analysis; ${ }^{\dagger}$ the proportion of patients with liver failure is $100 \%$ for patients with major hepatectomy and $0 \%$ for those with minor hepatectomy (Fisher's exact test: $p<0.001$ )

In total, eight patients (1.0\%) died in the perioperative period after liver resection. Seven deaths happened within 90 days and one occurred at 101 days during hospital stay. The six men and two women had a median age of 64 (range 48-75) years. Perioperative blood transfusion (OR 14.00, 95\% Cl 2.74-71.51, p=0.002) was the sole factor related to increased mortality in univariable analysis. A trend was found for major liver resection (OR 6.56, 95\% Cl 0.77-55.90, p=0.085) and preoperative comorbidity (OR 7.84, 95\% Cl $0.92-66.65, p=0.059)$ as factors associated with increased postoperative mortality. Severe SD (OR 2.79, 95\% Cl 0.68-11.44, $p=0.155$ ), severe steatosis (OR 0.86, 95\% Cl 0.107.09, $p=0.887$ ), and steatohepatitis (OR 2.71, 95\% Cl 0.57-12.87, $p=0.210$ ) were not related to postoperative mortality.

Because steatohepatitis, but not severe steatosis, negatively affected postoperative short-term outcomes, lobular inflammation and hepatocellular ballooning were considered key factors for poor outcome. A subgroup analysis supported this hypothesis. In multivariable analyses, severe (grade 2-3) lobular inflammation was associated with increased overall postoperative morbidity (OR 2.22, 95\% Cl 1.48-3.34, $\mathrm{p}=0.001$ ) and liver surgery-specific morbidity (OR 3.35, 95\% Cl 2.11-5.32, $\mathrm{p}<0.001$ ), but not major morbidity (OR 1.63, 95\% Cl 0.85-3.10, p=0.138). In contrast, neither severe steatosis (>33\%) nor 
the presence of hepatocellular ballooning (grade 1-2) were associated with an increased complication rate in all multivariable analyses.

Associations between several preoperative variables and the occurrence of severe SD, severe steatosis, and steatohepatitis are summarized in Figure 3.3 and Appendix 3.7. Oxaliplatin (OR 2.74, 95\% Cl 1.67-4.49, p<0.001) was related increased occurrence of severe SD in multivariable analysis, whereas the addition of bevacizumab was related to a twofold decrease in the occurrence of severe SD in patients who received oxaliplatin (OR $0.50,95 \% \mathrm{Cl} 0.30-0.82, \mathrm{p}=0.006$ ) when the analysis was adjusted solely by database source. Patients with severe steatosis showed a decreased incidence of severe SD (OR $0.44,95 \% \mathrm{Cl} 0.24-0.83, \mathrm{p}=0.011$ ) and vice versa (OR 0.36, 95\% $\mathrm{Cl} 0.15-0.88, \mathrm{p}=0.025$ ). $\mathrm{BMI}$ was related to an increase in severe steatosis (OR 1.15, 95\% Cl 1.08-1.21, $\mathrm{p}<0.001$ ), whereas the incidence of severe steatosis was decreased in patients with portal venous embolization (OR 0.29, 95\% Cl 0.08-1.00, $p=0.050$ ). Only severe steatosis was significantly associated with an increased occurrence of steatohepatitis (OR 15.09, 95\% Cl 6.25-36.45, $p<0.001)$.
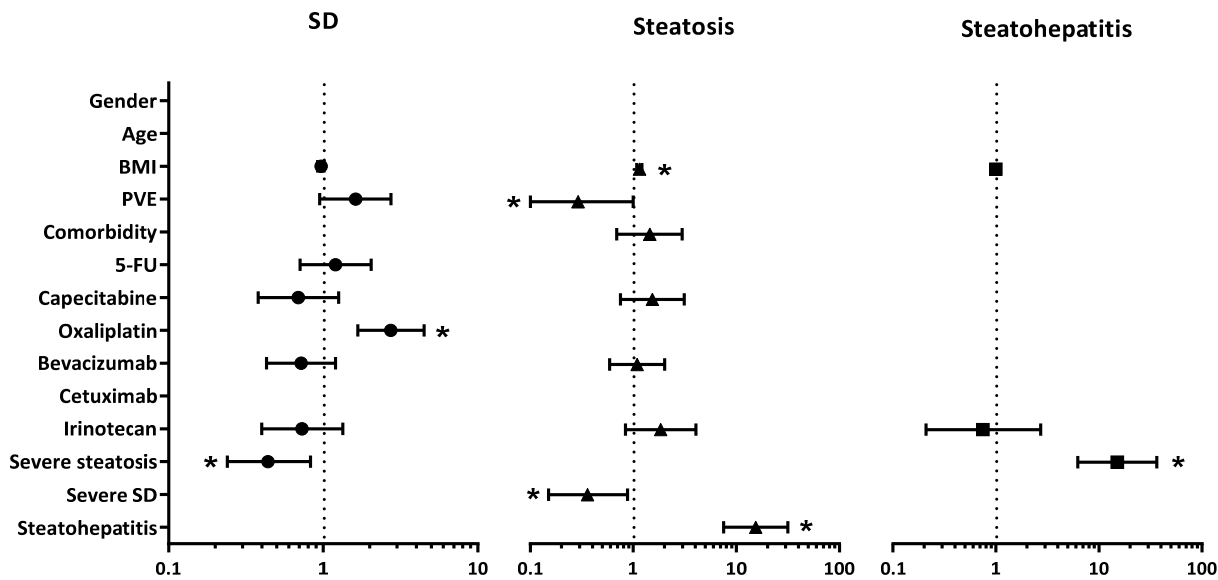

Figure 3.3 Multivariable analysis of influence of risk factors on liver injury.

Data are depicted as OR (symbol) with 95\% confidence intervals (bars) based on results of multivariable analysis; BMI, body mass index; PVE, portal venous embolization; 5-FU, 5fluorouracil; SD, sinusoidal dilatation; asterisks indicate $p$-value $\leq 0.05$. 


\section{Discussion}

In this study, an increase in postoperative major morbidity and liver surgery-specific complications after partial hepatectomy in patients with sinusoidal dilatation and steatohepatitis were observed, whereas steatosis was associated with a decreased occurrence of complications. Moreover, postoperative liver failure occurred more often in patients with severe SD. With respect to steatohepatitis, lobular inflammation, but not severe steatosis and hepatocellular ballooning, was strongly linked to increased postoperative morbidity. Oxaliplatin-based chemotherapy was the sole factor independently associated with an increase in the occurrence of severe SD, whereas a decrease in the occurrence of severe SD was seen with the addition of bevacizumab. Moreover, an inverse relationship between severe SD and severe steatosis was found.

Mechanisms for the negative influence of severe SD on major morbidity are unknown, although preoperative hepatic dysfunction, impairment of liver regeneration, Kupffer cell dysfunction, enhanced blood loss due to haemorrhagic pools, fragility of the liver, and increased hepatocellular necrosis as seen in human and animal models can all be reasons for poor liver function and an increased complication rate after severe SD. ${ }^{39,40}$ However, since the p-value was borderline significant, the effect of severe SD on major morbidity must be interpreted with caution.

The progression of steatohepatitis is estimated by the so-called NAS score, which is composed of scores for steatosis, lobular inflammation, and hepatocellular ballooning. ${ }^{27}$ Multivariable subgroup analyses concerning the influence of these separate NASsubcategories on postoperative outcome showed a detrimental influence of lobular inflammation on all outcomes, whereas severe steatosis and hepatocellular ballooning had no effect. The mechanism behind steatohepatitis being discriminant for postoperative short-term outcomes is uncertain. Electron microscopy revealed that mitochondrial structural defects in hepatocytes are correlated with steatohepatitis, but not with steatosis. ${ }^{41}$ Moreover, the ability of the liver to recover from ATP depletion was severely impaired, ${ }^{42}$ liver regeneration was diminished, ${ }^{43}$ and humoral and cellular immune responses in reaction to enhanced oxidative stress were found in patients with steatohepatitis. These factors may be accountable for postoperative complications.

Severe steatosis did not significantly influence short-term overall morbidity, major morbidity, or mortality after partial hepatectomy in this study, which is in line with previous reports ${ }^{44,45}$ but in contrast to other studies. ${ }^{46,47}$ Patients with severe steatosis even showed a decreased occurrence of liver surgery-specific complication. This might 
be explained by surgeons being more careful during surgery when observing a severely steatotic ("yellow") liver. However, with a p-value of 0.049 , this evidence needs to be validated by future research.

Patients who received oxaliplatin showed an increased occurrence of severe SD compared to patients who did not receive oxaliplatin (29.6\% vs. $14.0 \%$ ). This is in line with former studies which showed a similarly high occurrence of severe SD after oxaliplatin-based regimens. ${ }^{10,17}$ The addition of bevacizumab, an angiogenesis inhibitor, to oxaliplatin-based chemotherapy has been associated with a decreased incidence of SD. ${ }^{48}$ Indeed, when the analysis was restricted to the population that received oxaliplatin-based treatment, bevacizumab was associated with a remarkably decreased occurrence of severe SD. Although mechanisms underpinning those observations remain unclear, activation of vascular endothelial growth factor and coagulation pathways in oxaliplatin-related SD might be accountable. ${ }^{49}$

Importantly, severe steatosis was linked to a decreased occurrence of severe SD and vice versa, raising the possibility that these events are mutually exclusive. Several phenomena could underlie this observation. First, mechanical pressure exerted by fatladen, swollen hepatocytes may distort the hepatic microvasculature. In mice with severe steatosis, a decrease in sinusoidal perfusion, loss of fenestrae, and narrowing of the sinusoidal lumen were observed. ${ }^{50}$ Spatially, sinusoidal dilatation may therefore not develop. Conversely, atrophied hepatocellular plates in severe SD may render these hepatocytes incapable of fatty acid (FA) uptake. Alternatively, histological assessment may be more challenging in a liver affected by both severe SD and steatosis, increasing the likelihood of misclassification of either one of these injury types. However, several independently contacted pathologists with hepatobiliary expertise considered this probability very small. Although the reduction in sinusoidal dilation in patients with severe steatosis is interesting, central pathology review should be performed to verify this finding.

Apart from oxaliplatin, the preoperative performance of PVE showed to be associated with an increased occurrence of severe SD. Previous studies already acknowledged a possible influence of PVE on the development of vascular injury, probably by the induction of ischemia. ${ }^{51}$ We hypothesize that the hepatic artery buffer response after PVE might play an even more profound role as shown by the induction of microvascular remodeling and sinusoidal dilatation in the embolized lobe after portal branch ligation in a rodent model. ${ }^{52}$ However, PVE-induced histopathological changes in the embolized 
lobe might not be indicative for the non-embolized lobe, and may thus not indubitably affect liver histology and function after resection. ${ }^{53}$

In the present study it is observed that the occurrence of severe SD is lower in patients receiving additional bevacizumab compared to those receiving oxaliplatin alone. Severe SD was shown to be associated with an increased complication rate and vice versa. Although the effect of co-administration with bevacizumab on surgical outcome could not be investigated directly, adding bevacizumab might provide an advantageous effect on postoperative outcome in patients treated with oxaliplatin.

Parenchymal damage due to chemotherapy can be preoperatively diagnosed by radiological and biochemical tools, as reviewed recently in detail by our group. ${ }^{54}$ Despite (experimental) research focusing on CALI, little evidence is available for the treatment of CALI in the human setting. ${ }^{54}$ When liver injury is confirmed preoperatively, surgeons are thus advised to adapt surgical management to prevent complications. In the present study, the transfusion of packed red blood cells was associated with an increased postoperative complication rate, which is in line with previous literature. ${ }^{55}$ Central venous pressure should be low during surgery to prevent excessive blood loss. Moreover, the performance of a major hepatectomy was confirmed to be associated with an increased postoperative complication rate, which encourages minimizing the resection volume. Performing wedge resections instead of a hemihepatectomy, and the use of radiofrequency ablation might be beneficial when feasible.

To our knowledge, this is the first study demonstrating both the effect of chemotherapy on liver injury and the subsequent effect of liver injury on short-term postoperative outcome in a large, multicentre patient cohort. Some limitations of this study should be discussed. NRH has recently caused concern because of its relationship to increased postoperative morbidity. ${ }^{11}$ Although analysis of the effect of $\mathrm{NRH}$ on postoperative outcome would have been of interest, this was not possible due to unavailability of data. Establishment of included databases before or around the year 2013, when NRH did not yet gain the attention it deserves, may be the reason for missing data. With respect to the negative influence of $\mathrm{NRH}$ on postoperative outcomes, it is recommended to include $\mathrm{NRH}$ in future studies when exploring the relationship between CALI and postoperative outcomes. Next, data on the interval between cessation of chemotherapy and partial hepatectomy, as well as the number of administered cycles, were not available for every study cohort. Therefore, the influence of these factors on the occurrence of CALI and short-term complications could not be evaluated. Next, central review of all histopathology slides would have strengthened the paper substantially. Unfortunately, 
this was not feasible due to logistical reasons. It must be highlighted nonetheless, that all sections were reviewed by local pathologists with hepatobiliary expertise and assessed according to uniform, well-established, and globally accepted scoring systems for SD, steatosis, and steatohepatitis. Lastly, since no randomized controlled trials exist on the topic, mainly retrospective cohort studies were included in this review. Despite this limitation, all included studies showed low risk of bias and nearly all original data could be retained, making it the most comprehensive multicentre data cohort currently available.

\section{Conclusion}

This study demonstrated that after partial hepatectomy, severe SD and steatohepatitis were related to increased postoperative major morbidity and liver surgery-specific complications. Regarding steatohepatitis, lobular inflammation, but not steatosis or hepatocellular ballooning, was associated with increased postoperative overall morbidity and liver surgery-specific complications. Furthermore, severe SD was linked to an increased occurrence of postoperative liver failure. Lastly, oxaliplatin was strongly related to an increased occurrence of severe SD, and the addition of bevacizumab to oxaliplatin-based regimen reduced the occurrence of severe SD. Considering the negative relationship between CALI and postoperative morbidity, it is advised to adapt surgical management when CALI is diagnosed. Moreover, with decreased chemotherapy responsiveness, ${ }^{28,56}$ shortened overall survival, ${ }^{57}$ and increasing doubts about the usefulness of neoadjuvant chemotherapy in certain patient groups, ${ }^{58}$ one could even speculate that some patients would benefit from immediate resection instead of neoadjuvant chemotherapeutical treatment. Prospective registrations such as the ALPPS-registry ${ }^{59}$ provide a way to obtain a higher level of evidence on this topic. 


\section{References}

1. Ferlay J, Soerjomataram I, Dikshit R, Eser S, Mathers C, Rebelo M et al. Cancer incidence and mortality worldwide: sources, methods and major patterns in GLOBOCAN 2012. Int J Cancer 2015;136(5): E359-86.

2. Leonard GD, Brenner B, Kemeny NE. Neoadjuvant chemotherapy before liver resection for patients with unresectable liver metastases from colorectal carcinoma. J Clin Oncol 2005;23(9): 2038-48.

3. Kanas GP, Taylor A, Primrose JN, Langeberg WJ, Kelsh MA, Mowat FS et al. Survival after liver resection in metastatic colorectal cancer: review and meta-analysis of prognostic factors. Clin Epidemiol 2012;4: 283-301.

4. Scheele J. Hepatectomy for liver metastases. Br J Surg 1993;80(3): 274-6.

5. Tanaka K, Adam R, Shimada H, Azoulay D, Levi F, Bismuth H. Role of neoadjuvant chemotherapy in the treatment of multiple colorectal metastases to the liver. Br J Surg 2003;90(8): 963-9.

6. Adam R, Wicherts DA, de Haas RJ, Ciacio O, Levi F, Paule B et al. Patients with initially unresectable colorectal liver metastases: is there a possibility of cure? J Clin Oncol 2009;27(11): 1829-35.

7. Nordlinger B, Van Cutsem E, Rougier P, Kohne CH, Ychou M, Sobrero A et al. Does chemotherapy prior to liver resection increase the potential for cure in patients with metastatic colorectal cancer? A report from the European Colorectal Metastases Treatment Group. Eur J Cancer 2007;43(14): 2037-45.

8. Robinson SM, Wilson CH, Burt AD, Manas DM, White SA. Chemotherapy-associated liver injury in patients with colorectal liver metastases: a systematic review and meta-analysis. Ann Surg Oncol 2012;19(13): 4287-99.

9. Zorzi D, Laurent A, Pawlik TM, Lauwers GY, Vauthey JN, Abdalla EK. Chemotherapy-associated hepatotoxicity and surgery for colorectal liver metastases. Br J Surg 2007;94(3): 274-86.

10. Rubbia-Brandt L, Lauwers GY, Wang H, Majno PE, Tanabe K, Zhu AX et al. Sinusoidal obstruction syndrome and nodular regenerative hyperplasia are frequent oxaliplatin-associated liver lesions and partially prevented by bevacizumab in patients with hepatic colorectal metastasis. Histopathology 2010;56(4): 430-9.

11. Vigano L, Rubbia-Brandt L, De Rosa G, Majno P, Langella S, Toso C et al. Nodular Regenerative Hyperplasia in Patients Undergoing Liver Resection for Colorectal Metastases After Chemotherapy: Risk Factors, Preoperative Assessment and Clinical Impact. Ann Surg Oncol 2015;22(13): 4149-57.

12. Ribero D, Wang $H$, Donadon $M$, Zorzi D, Thomas MB, Eng $C$ et al. Bevacizumab improves pathologic response and protects against hepatic injury in patients treated with oxaliplatin-based chemotherapy for colorectal liver metastases. Cancer 2007;110(12): 2761-7.

13. Vauthey JN, Pawlik TM, Ribero D, Wu TT, Zorzi D, Hoff PM et al. Chemotherapy regimen predicts steatohepatitis and an increase in 90-day mortality after surgery for hepatic colorectal metastases. J Clin Oncol 2006;24(13): 2065-72.

14. Khan AZ, Morris-Stiff G, Makuuchi M. Patterns of chemotherapy-induced hepatic injury and their implications for patients undergoing liver resection for colorectal liver metastases. J Hepatobiliary Pancreat Surg 2009;16(2): 137-44.

15. Nakano H, Oussoultzoglou E, Rosso E, Casnedi S, Chenard-Neu MP, Dufour P et al. Sinusoidal injury increases morbidity after major hepatectomy in patients with colorectal liver metastases receiving preoperative chemotherapy. Ann Surg 2008;247(1): 118-24.

16. Pilgrim $\mathrm{CH}$, Satgunaseelan L, Pham A, Murray $\mathrm{W}$, Link E, Smith $\mathrm{M}$ et al. Correlations between histopathological diagnosis of chemotherapy-induced hepatic injury, clinical features, and perioperative morbidity. HPB 2012;14(5): 333-40.

17. Vigano L, Ravarino N, Ferrero A, Motta M, Torchio B, Capussotti L. Prospective evaluation of accuracy of liver biopsy findings in the identification of chemotherapy-associated liver injuries. Arch Surg 2012;147(12): 1085-91.

18. Aloia T, Sebagh M, Plasse M, Karam V, Levi F, Giacchetti S et al. Liver histology and surgical outcomes after preoperative chemotherapy with fluorouracil plus oxaliplatin in colorectal cancer liver metastases. J Clin Oncol 2006;24(31): 4983-90.

19. Komori H, Beppu T, Baba Y, Horino K, Imsung C, Masuda T et al. Histological liver injury and surgical outcome after FOLFOX followed by a hepatectomy for colorectal liver metastases in Japanese patients. Int J Clin Oncol 2010;15(3): 263-70. 
20. Pawlik TM, Olino K, Gleisner AL, Torbenson M, Schulick R, Choti MA. Preoperative chemotherapy for colorectal liver metastases: impact on hepatic histology and postoperative outcome. J Gastrointest Surg 2007;11(7): 860-8.

21. Sahajpal A, Vollmer CM, Dixon E, Chan EK, Wei A, Cattral MS et al. Chemotherapy for colorectal cancer prior to liver resection for colorectal cancer hepatic metastases does not adversely affect peri-operative outcomes. J Surg Oncol 2007;95(1): 22-7.

22. Makowiec F, Mohrle S, Neeff H, Drognitz O, Illerhaus G, Opitz OG et al. Chemotherapy, liver injury, and postoperative complications in colorectal liver metastases. J Gastrointest Surg 2011;15(1): 153-64.

23. Kandutsch S, Klinger M, Hacker S, Wrba F, Gruenberger B, Gruenberger T. Patterns of hepatotoxicity after chemotherapy for colorectal cancer liver metastases. Eur J Surg Oncol 2008;34(11): 1231-6.

24. Moher D, Liberati A, Tetzlaff J, Altman DG, Group P. Preferred reporting items for systematic reviews and meta-analyses: the PRISMA statement. Ann Intern Med 2009;151(4): 264-9, W264.

25. Stroup DF, Berlin JA, Morton SC, Olkin I, Williamson GD, Rennie D et al. Meta-analysis of observational studies in epidemiology: a proposal for reporting. Meta-analysis Of Observational Studies in Epidemiology (MOOSE) group. JAMA 2000;283(15): 2008-12.

26. Rubbia-Brandt L, Audard V, Sartoretti P, Roth AD, Brezault C, Le Charpentier M et al. Severe hepatic sinusoidal obstruction associated with oxaliplatin-based chemotherapy in patients with metastatic colorectal cancer. Ann Oncol 2004;15(3): 460-6.

27. Kleiner DE, Brunt EM, Van Natta M, Behling C, Contos MJ, Cummings OW et al. Design and validation of a histological scoring system for nonalcoholic fatty liver disease. Hepatology 2005;41(6): 1313-21.

28. Vreuls CP, Van Den Broek MA, Winstanley A, Koek GH, Wisse E, Dejong CH et al. Hepatic sinusoidal obstruction syndrome (SOS) reduces the effect of oxaliplatin in colorectal liver metastases. Histopathology 2012;61(2): 314-8.

29. van Mierlo KMC, Zhao J, Kleijnen J, Rensen SS, Schaap FG, Dejong CHC et al. The influence of chemotherapy-associated sinusoidal dilatation on short-term outcome after partial hepatectomy for colorectal liver metastases: A systematic review with meta-analysis. Surg Oncol 2016;25(3): 298-307.

30. Brunt EM, Kleiner DE, Wilson LA, Belt P, Neuschwander-Tetri BA, Network NCR. Nonalcoholic fatty liver disease (NAFLD) activity score and the histopathologic diagnosis in NAFLD: distinct clinicopathologic meanings. Hepatology 2011;53(3): 810-20.

31. Dindo D, Demartines N, Clavien PA. Classification of surgical complications: a new proposal with evaluation in a cohort of 6336 patients and results of a survey. Ann Surg 2004;240(2): 205-13.

32. van den Broek MA, van Dam RM, van Breukelen GJ, Bemelmans MH, Oussoultzoglou E, Pessaux P et al. Development of a composite endpoint for randomized controlled trials in liver surgery. $\mathrm{Br} J \mathrm{Surg}$ 2011;98(8): 1138-45.

33. Balzan S, Belghiti J, Farges O, Ogata S, Sauvanet A, Delefosse D et al. The "50-50 criteria" on postoperative day 5: an accurate predictor of liver failure and death after hepatectomy. Ann Surg 2005;242(6): 824-8, discussion 828-9.

34. Couinaud C. Liver anatomy: portal (and suprahepatic) or biliary segmentation. Dig Surg 1999;16(6): 459-67.

35. Hayden JA, van der Windt DA, Cartwright JL, Cote P, Bombardier C. Assessing bias in studies of prognostic factors. Ann Intern Med 2013;158(4): 280-6.

36. Hayden JA, Cote $P$, Bombardier C. Evaluation of the quality of prognosis studies in systematic reviews. Ann Intern Med 2006;144(6): 427-37.

37. Debray TP, Moons KG, Abo-Zaid GM, Koffijberg H, Riley RD. Individual participant data meta-analysis for a binary outcome: one-stage or two-stage? PLoS One 2013;8(4): e60650.

38. Abo-Zaid G, Guo B, Deeks JJ, Debray TP, Steyerberg EW, Moons KG et al. Individual participant data meta-analyses should not ignore clustering. J Clin Epidemiol 2013;66(8): 865-73 e864.

39. Schiffer E, Frossard JL, Rubbia-Brandt L, Mentha G, Pastor CM. Hepatic regeneration is decreased in a rat model of sinusoidal obstruction syndrome. J Surg Oncol 2009;99(7): 439-46.

40. Hubert C, Dahrenmoller C, Marique L, Jabbour N, Gianello P, Leclercq I. Hepatic regeneration in a rat model is impaired by chemotherapy agents used in metastatic colorectal cancer. Eur J Surg Oncol 2015;41(11): 1471-8. 
41. Sanyal AJ, Campbell-Sargent C, Mirshahi F, Rizzo WB, Contos MJ, Sterling RK et al. Nonalcoholic steatohepatitis: association of insulin resistance and mitochondrial abnormalities. Gastroenterology 2001;120(5): 1183-92.

42. Cortez-Pinto H, Chatham J, Chacko VP, Arnold C, Rashid A, Diehl AM. Alterations in liver ATP homeostasis in human nonalcoholic steatohepatitis: a pilot study. JAMA 1999;282(17): 1659-64.

43. Yang SQ, Lin HZ, Mandal AK, Huang J, Diehl AM. Disrupted signaling and inhibited regeneration in obese mice with fatty livers: implications for nonalcoholic fatty liver disease pathophysiology. Hepatology 2001;34(4 Pt 1): 694-706.

44. Wiggans MG, Lordan JT, Shahtahmassebi G, Aroori S, Bowles MJ, Stell DA. The Interaction between Diabetes, Body Mass Index, Hepatic Steatosis, and Risk of Liver Resection: Insulin Dependent Diabetes Is the Greatest Risk for Major Complications. HPB Surg 2014;2014: 586159.

45. Reddy SK, Marsh JW, Varley PR, Mock BK, Chopra KB, Geller DA et al. Underlying steatohepatitis, but not simple hepatic steatosis, increases morbidity after liver resection: a case-control study. Hepatology 2012;56(6): 2221-30.

46. McCormack L, Petrowsky H, Jochum W, Furrer K, Clavien PA. Hepatic steatosis is a risk factor for postoperative complications after major hepatectomy: a matched case-control study. Ann Surg 2007;245(6): 923-30.

47. Kooby DA, Fong Y, Suriawinata A, Gonen M, Allen PJ, Klimstra DS et al. Impact of steatosis on perioperative outcome following hepatic resection. J Gastrointest Surg 2003;7(8): 1034-44.

48. van der Pool AE, Marsman HA, Verheij J, Ten Kate FJ, Eggermont AM, ljzermans JN et al. Effect of bevacizumab added preoperatively to oxaliplatin on liver injury and complications after resection of colorectal liver metastases. J Surg Oncol 2012;106(7): 892-7.

49. Rubbia-Brandt L, Tauzin S, Brezault C, Delucinge-Vivier C, Descombes P, Dousset B et al. Gene expression profiling provides insights into pathways of oxaliplatin-related sinusoidal obstruction syndrome in humans. Mol Cancer Ther 2011;10(4): 687-96.

50. Farrell GC, Teoh NC, McCuskey RS. Hepatic microcirculation in fatty liver disease. Anat Rec (Hoboken) 2008;291(6): 684-92.

51. Brouquet A, Benoist S, Julie C, et al. Risk factors for chemotherapy-associated liver injuries: A multivariate analysis of a group of 146 patients with colorectal metastases. Surgery 2009;145(4): 362-71.

52. Kollmar O, Corsten M, Scheuer C, Vollmar B, Schilling MK, Menger MD. Portal branch ligation induces a hepatic arterial buffer response, microvascular remodeling, normoxygenation, and cell proliferation in portal blood-deprived liver tissue. Am J Physiol Gastrointest Liver Physiol 2007;292(6): 1534-42.

53. Matsuo K, Murakami T, Kawaguchi D, et al. Histologic features after surgery associating liver partition and portal vein ligation for staged hepatectomy versus those after hepatectomy with portal vein embolization. Surgery 2016;159(5): 1289-98.

54. van Mierlo KM, Schaap FG, Dejong CH, Olde Damink SW. Liver resection for cancer: New developments in prediction, prevention and management of postresectional liver failure. J Hepato/ 2016; 65(6):1217-31

55. Farid SG, White A, Khan N, Toogood GJ, Prasad KR, Lodge JP. Clinical outcomes of left hepatic trisectionectomy for hepatobiliary malignancy. Br J Surg 2016;103(3): 249-56.

56. Vigano L, Capussotti L, De Rosa G, De Saussure WO, Mentha G, Rubbia-Brandt L. Liver Resection for Colorectal Metastases after Chemotherapy Impact of Chemotherapy-Related Liver Injuries, Pathological Tumor Response, and Micrometastases on Long-term Survival. Ann Surg 2013;258(5): 731-42.

57. TamandI D, Klinger M, Eipeldauer S, Herberger B, Kaczirek K, Gruenberger B et al. Sinusoidal obstruction syndrome impairs long-term outcome of colorectal liver metastases treated with resection after neoadjuvant chemotherapy. Ann Surg Oncol 2011;18(2): 421-30.

58. Khoo E, O'Neill S, Brown E, Wigmore SJ, Harrison EM. Systematic review of systemic adjuvant, neoadjuvant and perioperative chemotherapy for resectable colorectal-liver metastases. HPB 2016;18(6): 485-93.

59. Schadde E, Ardiles V, Robles-Campos R, Malago M, Machado M, Hernandez-Alejandro R et al. Early survival and safety of ALPPS: first report of the International ALPPS Registry. Ann Surg 2014;260(5): 829-36.

60. Gomez-Ramirez J, Martin-Perez E, Amat CG, Sanz IG, Bermejo E, Rodriguez A et al. Influence of presurgical chemotherapy on liver parenchyma and post-surgical outcome of patients subjected to hepatectomy due to colorectal carcinoma metastases. Cir Esp 2010;88(6): 404-12. 
61. Nam SJ, Cho JY, Lee HS, Choe G, Jang JJ, Yoon YS et al. Chemotherapy-associated hepatopathy in korean colorectal cancer liver metastasis patients: oxaliplatin-based chemotherapy and sinusoidal injury. Korean J Pathol 2012;46(1): 22-9.

62. Pessaux P, Marzano E, Casnedi S, Bachellier P, Jaeck D, Chenard MP. Histological and immediate postoperative outcome after preoperative cetuximab: case-matched control study. World J Surg 2010;34(11): 2765-72.

63. Soubrane O, Brouquet A, Zalinski S, Terris B, Brezault C, Mallet V et al. Predicting high grade lesions of sinusoidal obstruction syndrome related to oxaliplatin-based chemotherapy for colorectal liver metastases: correlation with post-hepatectomy outcome. Ann Surg 2010;251(3): 454-60.

64. Takamoto T, Hashimoto T, Sano K, Maruyama Y, Inoue K, Ogata S et al. Recovery of liver function after the cessation of preoperative chemotherapy for colorectal liver metastasis. Ann Surg Oncol 2010;17(10): 2747-55. 


\section{Appendix 3.1 Study protocol}

Systematic review of the influence of chemotherapy-associated liver injury on outcome after partial hepatectomy for colorectal liver metastases

J. Zhao, K. M. C. van Mierlo, S. S. Rensen, F. G. Schaap, B. Winkens, C. H. C. Dejong and S. W. M. Olde Damink

\section{Summary}

\section{Background}

Chemotherapeutic regimens (for example oxaliplatin and irinotecan-based), which are commonly administered in patients with colorectal liver metastases (CRLM), have been associated with undesirable so-called chemotherapy-associated liver injury (CALI, sinusoidal dilatation, steatosis and steatohepatitis). The influence of CALI on postoperative outcomes remains controversial.

\section{Objective}

We describe the protocol of a systematic review and individual participant data analysis, which aims to assess the impact of CALI on postoperative morbidity and mortality after partial hepatectomy.

\section{Methods}

The PRISMA and MOOSE guidelines will be followed for the conduct and report of this review. A literature search will be performed in MEDLINE (PubMed) and Embase, in addition to checking of references in papers found. The search will be limited to studies published between 1 January 2004 and 31 December 2013. No restrictions of language will be applied in the search strategy. We will include studies of any design (except case reports, comments, editorials and reviews) investigating the influence of CALI on postoperative morbidity and mortality within the population of adult patients (aged over 18 years) who underwent partial hepatectomy for colorectal liver metastases. The risk of bias of included papers will be assessed using the Quality in Prognosis Studies (QUIPS) tool for prognosis studies. Corresponding authors of included studies will be contacted for sharing the original data of the patient cohort. The raw data will then be recoded, redefined, pooled and analysed using binary logistic regression models.

\section{Ethics and dissemination}

No ethical issues are foreseen because (a) the data from each included study were already published in the literature; (b) each of the included studies obtained local ethics committee approval; (c) the project uses anonymized data of individuals who cannot be identified. The results will be submitted to a peer-reviewed journal. 


\section{Introduction}

Colorectal cancer is the third most common cancer worldwide, affecting over 1.3 million patients annually. ${ }^{1}$ Approximately 50 per cent of these patients develop colorectal liver metastases (CRLM). ${ }^{2}$ Although liver resection provides the best prospect of cure, only 10-30 per cent of patients with liver metastases are eligible for hepatic surgery. ${ }^{2}$ Inoperability is mainly caused by patient morbidity in combination with number and size of hepatic tumours. For patients with tumours deemed unresectable, systemic chemotherapy can prolong survival, and allow potential future hepatic resection.,

For decades, 5-fluorouracil was the sole active chemotherapeutic agent for CRLM. This has changed markedly in the new millennium: with the approval of irinotecan, oxaliplatin and humanized monoclonal antibodies, many more patients with initially unresectable CRLM became eligible for liver resection. ${ }^{5,6}$ Although response rates are convincing, ${ }^{3}$ administration of oxaliplatin and/or irinotecan-based chemotherapeutic agents has been associated with detrimental side-effects in the form of liver injury. ${ }^{7}$

Chemotherapy-associated liver injury (CALI) is often reported in patients with CRLM, and appears to be regimen-specific. For instance, oxaliplatin treatment is associated with sinusoidal obstruction syndrome (SOS), ${ }^{8}$ and increases the risk of nodular regenerative hyperplasia $(\mathrm{NRH}) .^{9}$ Co-administration of bevacizumab with oxaliplatin, however, has been reported to decrease both the incidence and severity of SOS and $\mathrm{NRH}^{8-10}$ Irinotecan-based regimens appear to be related to the development of steatohepatitis. $^{7,11,12}$ Importantly, because patients commonly receive several chemotherapeutic agents to offer optimal benefit in downsizing tumours, it is difficult to identify the specific agents responsible for injury of the hepatic parenchyma.

Whereas certain studies claim an evident negative correlation between CALI and postoperative outcome (postoperative morbidity, mortality, tumour response and/or long-term survival), ${ }^{13-15}$ others could not reproduce this. ${ }^{14-21}$ Therefore, it remains unclear whether CALI influences postoperative morbidity and mortality.

\section{Objectives}

The aim of this study is to distinguish whether sinusoidal dilatation (SD), steatosis and steatohepatitis are risk factors for increased morbidity and mortality rates after partial hepatectomy by performing a meta-analysis of individual participant data based on a systematic literature review. 


\section{Methods}

We developed the methods according to the PRISMA guidelines for conducting systematic reviews and meta-analyses ${ }^{22}$.

\section{Literature search}

The strategy for the literature search was developed within our systematic review group. J.Z. and K.v.M. will perform electronic searches in the databases of MEDLINE (PubMed) and Embase with the filters of publication dates (1 January 2004 to 31 December 2013). The publication date was determined to start from 2004 because the widely used criteria for scoring SD, steatosis and steatohepatitis were developed in $2004^{23}$ and $2005 .{ }^{24}$ We will not use language filters or any other filters to restrict the initial search. The search matrix includes four categories: liver resection, chemotherapy, tumour type and outcome. For the purpose of performing a more comprehensive search, we will not include the category of liver injury type (SD, steatosis, or steatohepatitis). Table S3.1 lists the full search strategy.

Table S3.1 Search strategy

\begin{tabular}{lllll}
\hline 1st category & 2nd category 2 & 3rd category & 4th category & Filters \\
\hline Liver resection & Chemotherapy & Tumour type & Outcomes & \\
Liver resection & Chemotherap* OR & Colorectal neoplasm & Morbidity OR & Dates: 01.01.2004- \\
OR Liver transection & Oxaliplatin OR & OR Colorectal cancer & Mortality OR Survival & 31.12.2013 \\
OR Hepatic & Irinotecan OR & OR Colorectal & OR Dead OR & Species: human \\
transection OR & FOLFOX OR & metastas* OR & Outcome OR & Age: >18 years \\
Hepatectomy & FOLFIRI OR & Colon cancer Liver & Recurrence OR Liver & \\
AND & CAPOX OR 5FU OR & metastas* & failure OR & \\
& Fluorouracil & AND & Regeneration OR & \\
& AND & & Hepatic insufficiency & \\
\hline
\end{tabular}

Asterisks indicate blurred searching in case of synonymous words

\section{Search protocol in PubMed and Embase}

((()(((liver resection) OR liver transection) OR hepatic transection) OR hepatectomy) AND ((()(((chemotherap*) OR oxaliplatin) OR irinotecan) OR folfox*) OR folfiri) OR

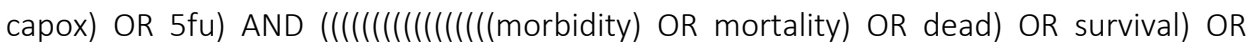
outcome) OR risk) OR recurrence) OR regeneration) OR liver failure) OR hepatic failure) OR hepatotoxicity) OR bleeding) OR hemorrhage) OR infection) OR hepatopathy) OR prognosis) AND (((()colorectal neoplasm*) OR colorectal cancer) OR colorectal metastas*) OR colon cancer) 
Filters

- Publication dates: 1 January 2004 to 31 December 2013

- Species: humans

- $\quad$ Ages: adults (>18 years)

Checking of reference lists

Two authors (J.Z. and K.v.M.) will manually check the reference lists of included studies for additional potential citations. Moreover, an exploration of a personal library that was established because of previous research on this topic will be performed. ${ }^{25}$

Identification and selection of studies

Studies identified by electronic searches and manual checking of reference lists will be imported into Endnote. The eligibility process will be conducted in two separate stages according to the inclusion criteria in Table S3.2.

1. Two authors (J.Z. and K.v.M.) will independently screen titles and abstracts of all non-duplicated papers and will exclude those not pertinent. A final list will be agreed with discrepancies resolved by consensus between the two authors. When consensus is not reached, a third author (S.O.D., S.R. or F.S.) will act as arbitrator. If any doubt about inclusion is dispelled, the article will proceed to the next stage.

2. The full-text version of the articles passing screening step 1 will be retrieved and assessed for eligibility by two authors (J.Z. and K.v.M.), independently. Discrepancies will be resolved by consensus between the two authors and, if needed, a third author (S.O.D., S.R. or F.S.) will act as arbitrator.

Table S3.2 Inclusion criteria

Articles published from January 2004 onwards

Study type: RCT, cohort study, case-control study, correlational study, case series and cross-sectional study. Studies of case reports, comments, editorials, or reviews will be excluded

Population: adult patients (>18 years) who underwent liver resection for colorectal liver metastases

Outcome: studies with description of postoperative short-term overall morbidity, liver surgery-specific complications, postoperative liver failure, or mortality ( $\leq 90$ days or in-hospital)

Pathological assessment: the severity of SOS was classified according to the criteria of Rubbia-Brandt et al. ${ }^{23}$ The severity of steatosis and steatohepatitis were classified according to the criteria of Kleiner et al. ${ }^{24}$

\section{Quality assessment}

Studies that meet the inclusion criteria will proceed to risk-of-bias assessment. As this is a systematic review for prognosis studies, we will adapt the QUIPS tool ${ }^{26,27}$ to assess the 
quality of the final included studies. The QUIPS tool includes six bias domains: participation, attrition, prognostic factor measurement, confounding measurement and account, outcome measurement, and analysis and reporting. For each of the six domains, responses to the prompting items will be taken together to inform the judgement of bias risk. Judgements will be made with consensus among two authors (J.Z. and K.v.M.). Any discrepancies will be resolved by consensus between the two authors. If this is not possible, another author (S.O.D., S.R. or F.S.) will make a judgement on the quality and act as an arbitrator.

\section{Data handling}

Corresponding authors from studies that fulfilled the inclusion criteria will be contacted by e-mail for collaboration and sharing of published raw data. Once the author agrees on sharing the data, a preconstructed Excel ${ }^{\mathrm{Tm}}$ file will be provided for the author to fill in the raw data. Each author will be asked to sign a specific data transfer agreements form, which assures careful handling of their data.

\section{Statistical analysis}

Shared raw data from the author will be pooled in SPSS ${ }^{\circledR}$ for Microsoft ${ }^{\circledR}$ Windows ${ }^{\circledR}$ (version 20.0). Patient characteristics will be compared using independent-samples $t$ test for numerical variables, and Pearson Chi-square test or Fisher's exact test, where appropriate, for categorical variables. The influence of preoperative chemotherapeutic agents on liver injury, and subsequently the effect of the liver injury on short-term postoperative outcome, will be analysed by applying one-step binary logistic regression models. This approach is considered most optimal because some postoperative outcomes (such as liver failure, intraabdominal abscess or mortality) are usually small probability events, and the one-step approach for pooled data allows the exact binomial distribution to be used and does not require continuity corrections when no events occur. $^{28,29}$

As for missing values, we will consult a statistician and perform multiple imputations if necessary. The number of imputations will depend on the percentage of missing values. Once multiple imputations are performed, subsequent sensitivity analyses comparing the analyses of complete cases versus multiple imputative cases will be conducted. Clustering of patients from different studies will be integrated as a separate co-variable ('database source') and included in binary logistic regression models in every analysis. ${ }^{28}$ A single variable together with database source will create the univariable model. A variable with $p \leq 0 \cdot 200$ in univariable analysis will be included for multivariable analysis. In addition, database source and known influencing co-variables (either well described in 
the literature or based on careful discussion and consensus between the authors) will be forced into the multivariable model. Unadjusted and adjusted odds ratios with 95\% confidence intervals will be calculated. $p \leq 0.050$ will be considered significant for all analyses.

\section{Data reporting}

Data will be reported according to the PRISM and MOOSE guidelines for systematic reviews. The manuscript will be reviewed and approved by all authors, and published in a peer-reviewed journal.

\section{References}

1. Ferlay J, Soerjomataram I, Dikshit R, Eser S, Mathers C, Rebelo M et al. Cancer incidence and mortality worldwide: sources, methods and major patterns in GLOBOCAN 2012. Int J Cancer 2015; 136: E359-86.

2. Kanas GP, Taylor A, Primrose JN, Langeberg WJ, Kelsh MA, Mowat FS et al. Survival after liver resection in metastatic colorectal cancer: review and meta-analysis of prognostic factors. Clin Epidemiol 2012; 4: 283-301.

3. Lam VW, Spiro C, Laurence JM, Johnston E, Hollands MJ, Pleass HC et al. A systematic review of clinical response and survival outcomes of downsizing systemic chemotherapy and rescue liver surgery in patients with initially unresectable colorectal liver metastases. Ann Surg Oncol 2012; 19: 1292-301.

4. Adam R, Delvart V, Pascal G, Valeanu A, Castaing D, Azoulay D et al. Rescue surgery for unresectable colorectal liver metastases downstaged by chemotherapy: a model to predict long-term survival. Ann Surg 2004; 240: 644-57.

5. Nordlinger B, Van Cutsem E, Rougier P, Köhne CH, Ychou M, Sobrero A et al. Does chemotherapy prior to liver resection increase the potential for cure in patients with metastatic colorectal cancer? A report from the European Colorectal Metastases Treatment Group. Eur J Cancer 2007; 43: 2037-45.

6. Van Cutsem E, Nordlinger B, Adam R, Köhne CH, Pozzo C, Poston G et al.; European Colorectal Metastases Treatment Group. Towards a pan-European consensus on the treatment of patients with colorectal liver metastases. Eur J Cancer 2006; 42: 2212-21.

7. Robinson SM, Wilson CH, Burt AD, Manas DM, White SA. Chemotherapy-associated liver injury in patients with colorectal liver metastases: a systematic review and meta-analysis. Ann Surg Oncol 2012; 19: 4287-99.

8. Rubbia-Brandt L, Lauwers GY, Wang H, Majno PE, Tanabe K, Zhu AX et al. Sinusoidal obstruction syndrome and nodular regenerative hyperplasia are frequent oxaliplatin-associated liver lesions and partially prevented by bevacizumab in patients with hepatic colorectal metastasis. Histopathology 2010; 56: 430-9.

9. Viganò L, Rubbia-Brandt L, De Rosa G, Majno P, Langella S, Toso C et al. Nodular regenerative hyperplasia in patients undergoing liver resection for colorectal metastases after chemotherapy: risk factors, preoperative assessment and clinical impact. Ann Surg Oncol 2015; 22: 4149-57.

10. Ribero $D$, Wang $H$, Donadon $M$, Zorzi D, Thomas MB, Eng $C$ et al. Bevacizumab improves pathologic response and protects against hepatic injury in patients treated with oxaliplatin-based chemotherapy for colorectal liver metastases. Cancer 2007; 110: 2761-7.

11. Khan AZ, Morris-Stiff G, Makuuchi M. Patterns of chemotherapy-induced hepatic injury and their implications for patients undergoing liver resection for colorectal liver metastases. J Hepatobiliary Pancreat Surg 2009; 16: 137-44.

12. Morris-Stiff G, Tan YM, Vauthey JN. Hepatic complications following preoperative chemotherapy with oxaliplatin or irinotecan for hepatic colorectal metastases. Eur J Surg Oncol 2008; 34: 609-14. 
13. Nakano H, Oussoultzoglou E, Rosso E, Casnedi S, Chenard-Neu MP, Dufour P et al. Sinusoidal injury increases morbidity after major hepatectomy in patients with colorectal liver metastases receiving preoperative chemotherapy. Ann Surg 2008; 247: 118-24.

14. Pilgrim CH, Satgunaseelan L, Pham A, Murray W, Link E, Smith M et al. Correlations between histopathological diagnosis of chemotherapy-induced hepatic injury, clinical features, and perioperative morbidity. HPB 2012; 14: 333-40.

15. Viganò L, Ravarino N, Ferrero A, Motta M, Torchio B, Capussotti L. Prospective evaluation of accuracy of liver biopsy findings in the identification of chemotherapy-associated liver injuries. Arch Surg 2012; 147: 1085-91.

16. Komori H, Beppu T, Baba Y, Horino K, Imsung C, Masuda T et al. Histological liver injury and surgical outcome after FOLFOX followed by a hepatectomy for colorectal liver metastases in Japanese patients. Int J Clin Oncol 2010; 15: 263-70.

17. Pawlik TM, Olino K, Gleisner AL, Torbenson M, Schulick R, Choti MA. Preoperative chemotherapy for colorectal liver metastases: impact on hepatic histology and postoperative outcome. J Gastrointest Surg 2007; 11: 860-8.

18. Sahajpal A, Vollmer CM, Dixon E, Chan EK, Wei A, Cattral MS et al. Chemotherapy for colorectal cancer prior to liver resection for colorectal cancer hepatic metastases does not adversely affect peri-operative outcomes. J Surg Oncol 2007; 95: 22-7.

19. Makowiec F, Möhrle S, Neeff H, Drognitz O, Illerhaus G, Opitz OG et al. Chemotherapy, liver injury, and postoperative complications in colorectal liver metastases. J Gastrointest Surg 2011; 15: 153-64.

20. Kandutsch S, Klinger M, Hacker S, Wrba F, Gruenberger B, Gruenberger T. Patterns of hepatotoxicity after chemotherapy for colorectal cancer liver metastases. Eur J Surg Oncol 2008; 34: 1231-6.

21. Viganò L, Capussotti L, De Rosa G, De Saussure WO, Mentha G, Rubbia-Brandt L. Liver resection for colorectal metastases after chemotherapy: impact of chemotherapy-related liver injuries, pathological tumor response, and micrometastases on long-term survival. Ann Surg 2013; 258: 731-40.

22. Moher D, Liberati A, Tetzlaff J, Altman DG; PRISMA Group. Preferred reporting items for systematic reviews and meta-analyses: the PRISMA statement. Ann Intern Med 2009; 151: 264-9, W264.

23. Rubbia-Brandt L, Audard V, Sartoretti P, Roth AD, Brezault C, Le Charpentier M et al. Severe hepatic sinusoidal obstruction associated with oxaliplatin-based chemotherapy in patients with metastatic colorectal cancer. Ann Oncol 2004; 15: 460-6.

24. Kleiner DE, Brunt EM, Van Natta M, Behling C, Contos MJ, Cummings OW et al. Design and validation of a histological scoring system for nonalcoholic fatty liver disease. Hepatology 2005; 41: 1313-21.

25. Vreuls CP, Van Den Broek MA, Winstanley A, Koek GH, Wisse E, Dejong CH et al. Hepatic sinusoidal obstruction syndrome (SOS) reduces the effect of oxaliplatin in colorectal liver metastases. Histopathology 2012; 61: 314-8.

26. Hayden JA, Côté $P$, Bombardier C. Evaluation of the quality of prognosis studies in systematic reviews. Ann Intern Med 2006; 144: 427-37.

27. Hayden JA, van der Windt DA, Cartwright JL, Côté P, Bombardier C. Assessing bias in studies of prognostic factors. Ann Intern Med 2013; 158: 280-6.

28. Abo-Zaid G, Guo B, Deeks JJ, Debray TP, Steyerberg EW, Moons KG et al. Individual participant data meta-analyses should not ignore clustering. J Clin Epidemiol 2013; 66: 865-73.e864

29. Debray TP, Moons KG, Abo-Zaid GM, Koffijberg H, Riley RD. Individual participant data meta-analysis for a binary outcome: one-stage or two-stage? PLOS ONE 2013; 8: e60650. 


\section{Appendix 3.2 Variables in multiple imputations}

\begin{tabular}{|c|c|c|c|c|c|}
\hline $\begin{array}{l}\text { Patient } \\
\text { cluster }\end{array}$ & General & Chemotherapy & Liver injury & Surgical factors & Outcome \\
\hline $\begin{array}{l}\text { Database } \\
\text { source }\end{array}$ & $\begin{array}{l}\text { Sex, age, BMI, } \\
\text { co-morbidity }\end{array}$ & $\begin{array}{l}\text { 5-Fluorouracil, } \\
\text { capecitabine, } \\
\text { irinotecan, } \\
\text { bevacizumab, } \\
\text { cetuximab, } \\
\text { oxaliplatin }\end{array}$ & $\begin{array}{l}\text { Sinusoidal } \\
\text { dilatation, } \\
\text { steatosis, } \\
\text { steatohepatitis }\end{array}$ & $\begin{array}{l}\text { Resection type, } \\
\text { PVE, pringle } \\
\text { manoeuvre, } \\
\text { transfusion of } \\
\text { packed RBCs }\end{array}$ & $\begin{array}{l}\text { Hospital stay, } \\
\text { overall morbidity, } \\
\text { complications } \\
\text { (minor/major), } \\
\text { liver surgery- } \\
\text { specific } \\
\text { complication, } \\
\text { mortality, liver } \\
\text { failure }\end{array}$ \\
\hline
\end{tabular}

PVE, portal vein embolization; RBC, red blood cell 


\section{Appendix 3.3 Flow chart summarizing final inclusion process}

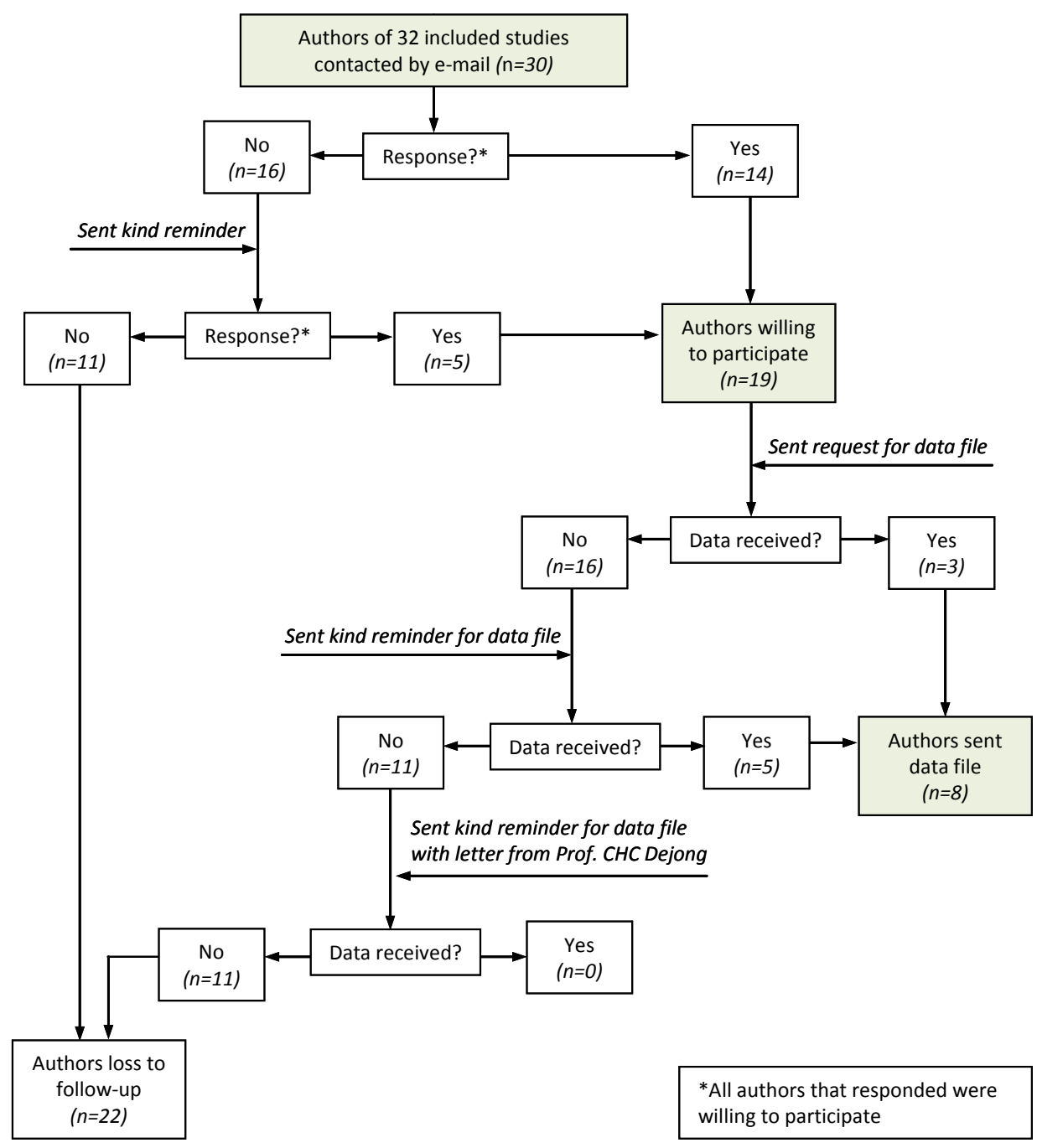




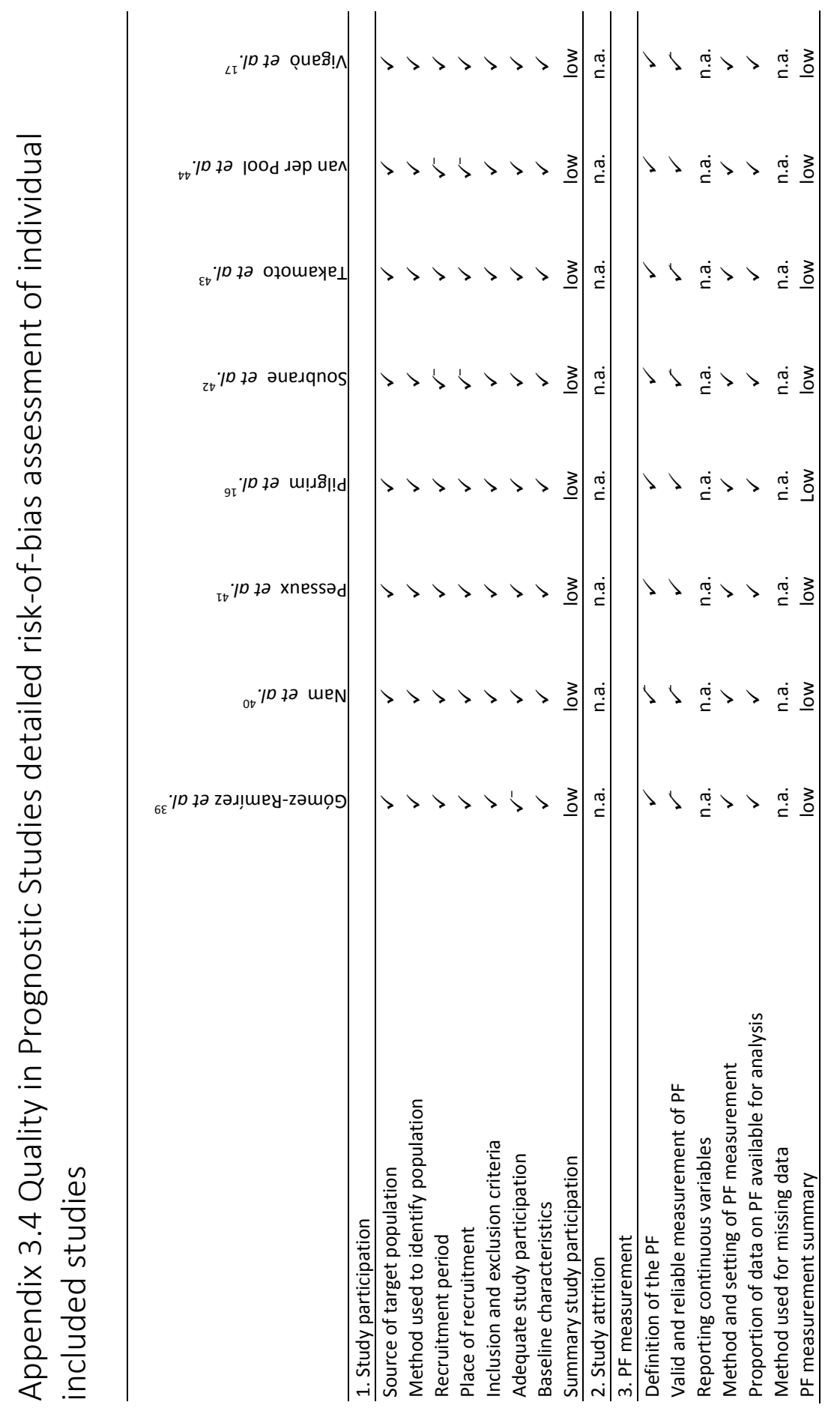




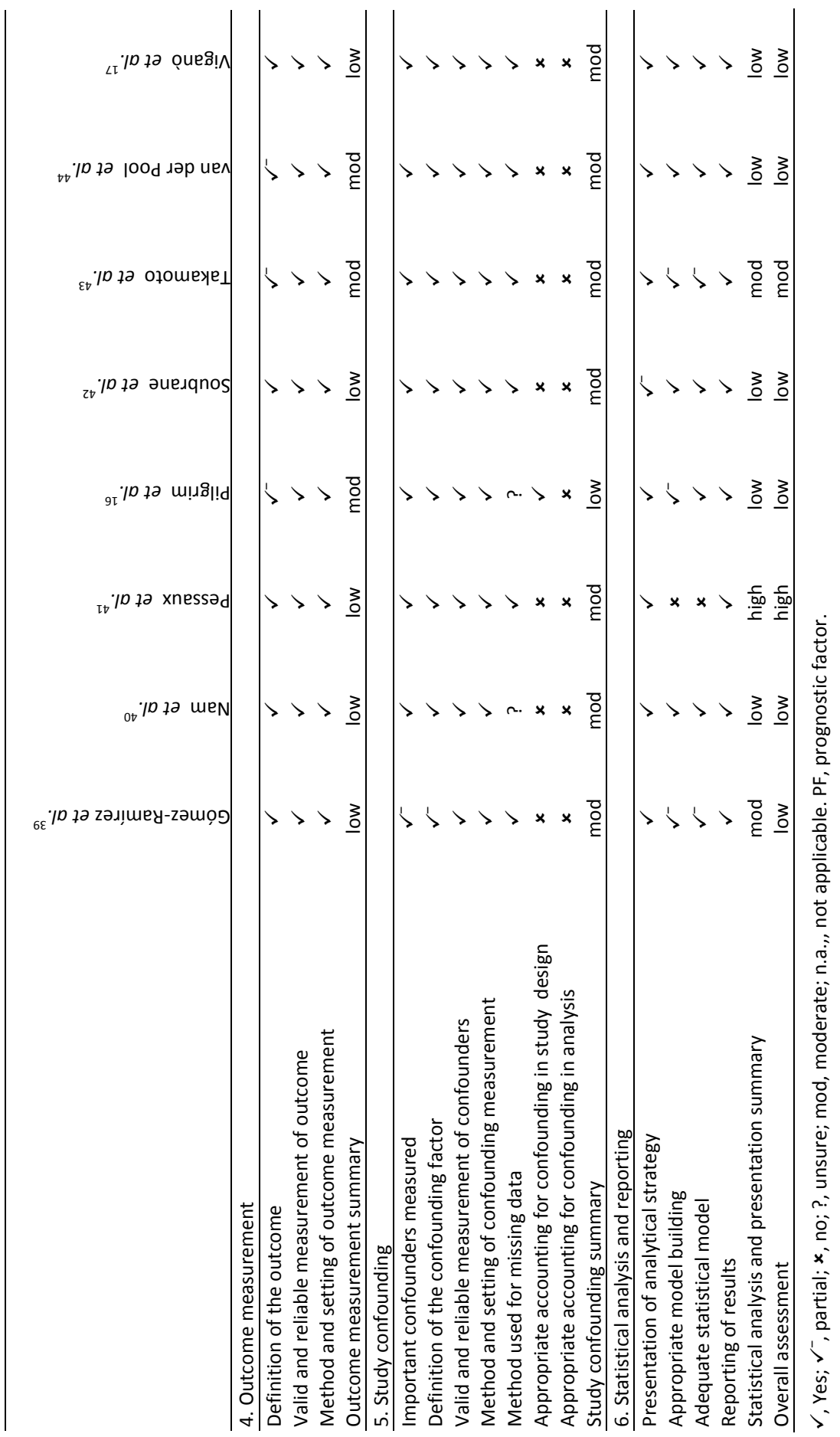


Appendix 3.5 Relationship between non-alcoholic fatty liver disease activity score and lobular inflammation

\begin{tabular}{lccccc}
\hline & \multicolumn{3}{c}{ Lobular inflammation (foci per $200 \times$ field) } & \\
\cline { 2 - 5 } NAS & 0 & $<2$ & $2-4$ & $>4$ & Total no. of patients \\
\hline 0 & 100 & 0 & 0 & 0 & 100 \\
1 & 59 & 130 & 0 & 0 & 189 \\
2 & 24 & 100 & 24 & 0 & 148 \\
3 & 6 & 46 & 39 & 11 & 102 \\
4 & 3 & 26 & 32 & 7 & 68 \\
5 & 0 & 5 & 7 & 4 & 16 \\
6 & 0 & 1 & 4 & 4 & 9 \\
7 & 0 & 0 & 2 & 1 & 3 \\
Total no. of patients & 192 & 308 & 108 & 27 & 635 \\
\hline
\end{tabular}

NAS, non-alcoholic fatty liver disease activity score 


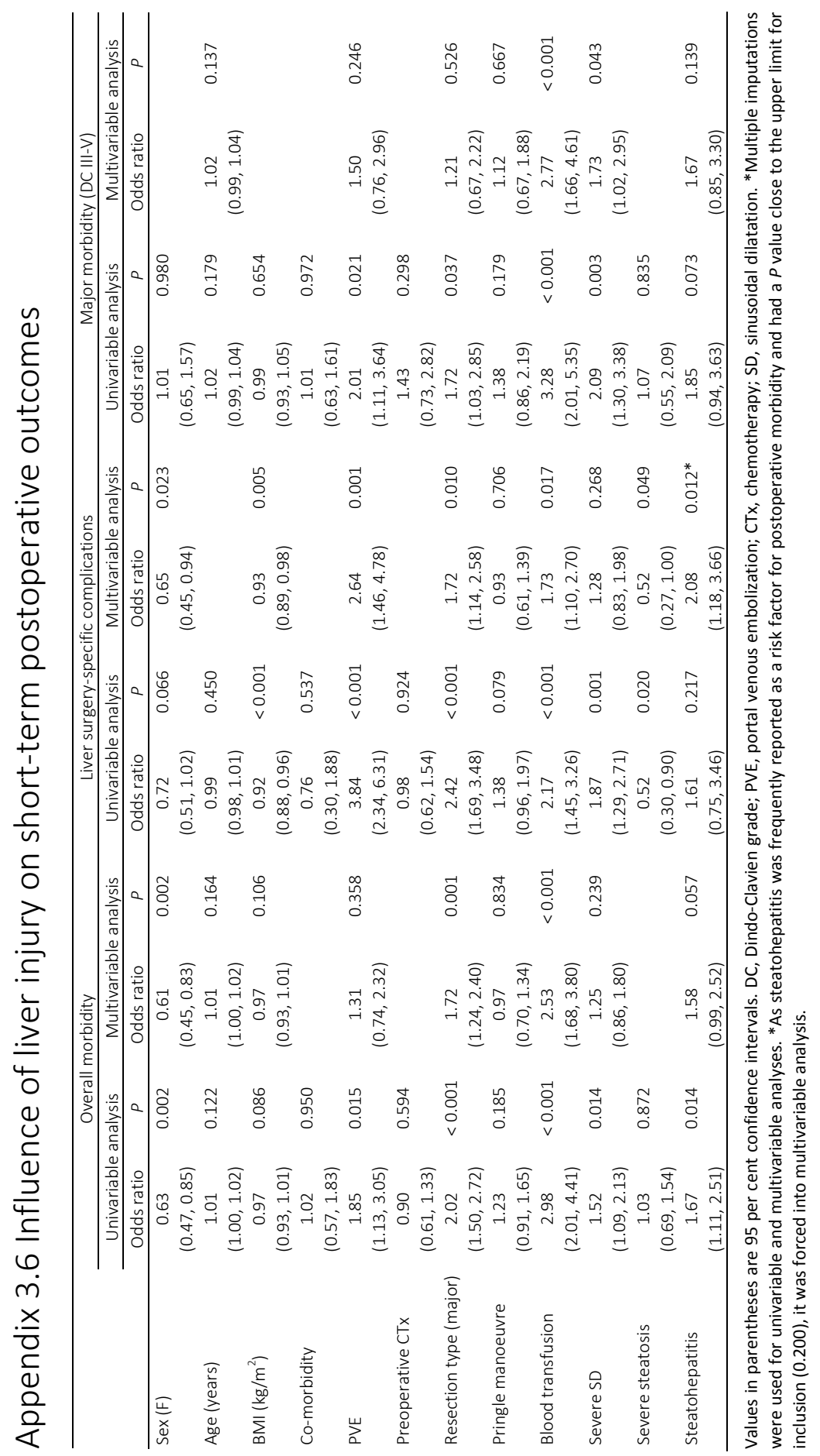




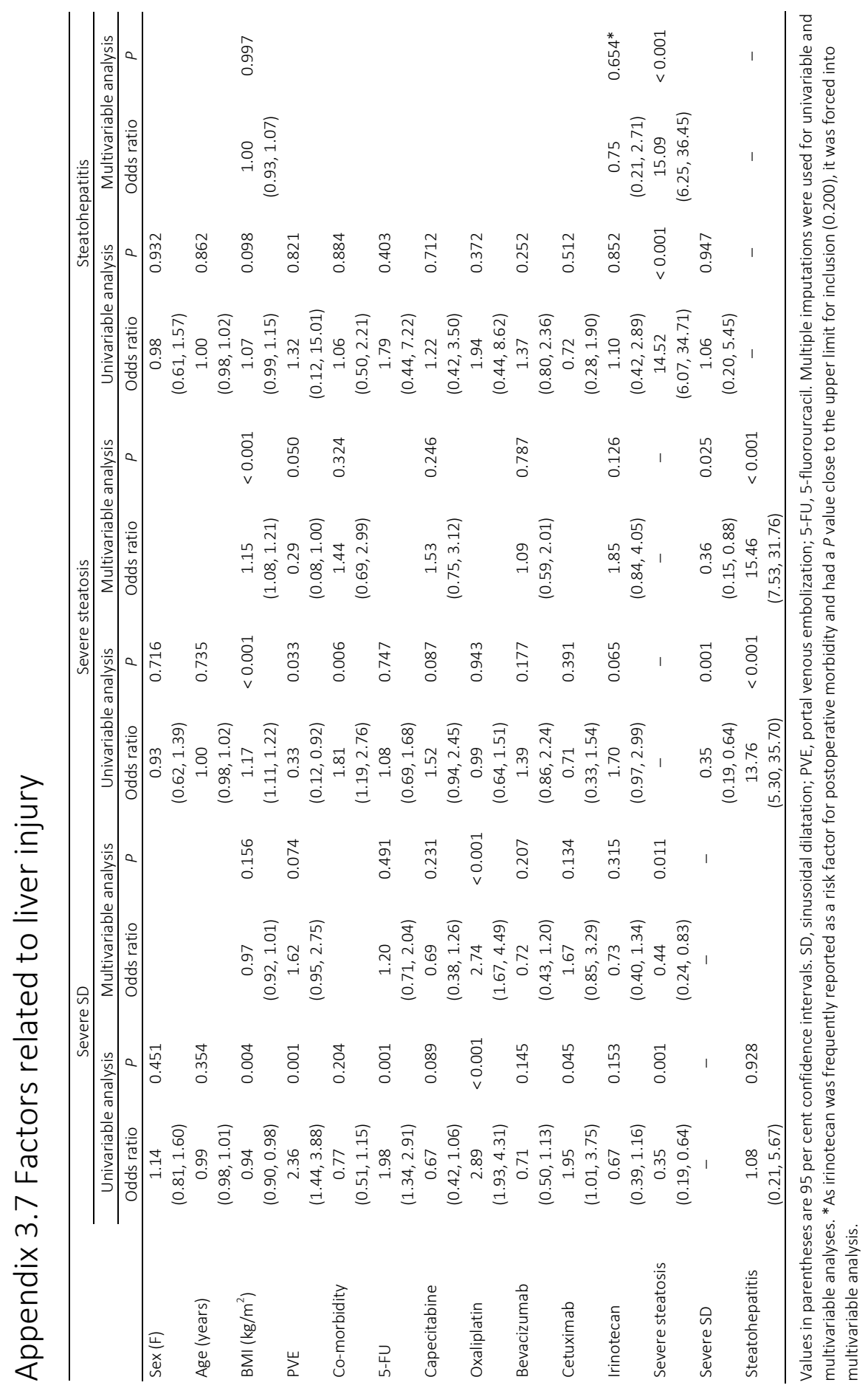




\title{
Chapter 4
}

\begin{abstract}
Impact of chemotherapy-associated liver injury on postoperative infectious complications after partial hepatectomy for colorectal liver metastases
\end{abstract}

Junfang Zhao, Pamir Sawo, Sander S. Rensen, Magriet M.J. Rouflart, Alison Winstanley, Celien P.H. Vreuls, Joanne Verheij, Kim M.C. van Mierlo, Toine M. Lodewick, Victor van Woerden, Frank H. van Tiel, Ronald M. van Dam, Cornelis H.C. Dejong, Steven W.M. Olde Damink Submitted 


\section{Abstract}

Introduction

Although postoperative infectious complications and chemotherapy-associated liver injury (CALI) are both major concerns for liver surgery in patients with colorectal liver metastases (CRLM), little is known regarding their relation. The aim of the current study was to investigate potential associations between CALI, postoperative infectious complications, and survival after partial hepatectomy.

\section{Methods}

Patients who underwent partial hepatectomy for CRLM between 2008 and 2014 were included. CALI was assessed histopathologically, focusing on steatosis, steatohepatitis, nodular regeneration, and sinusoidal dilatation. Infectious complications were prospectively monitored daily. Multivariable regression was performed, and results were depicted as odds ratio (OR) or hazard ratio (HR) with 95\% confidence interval (Cl).

\section{Results}

265 patients were included with a median period of follow-up of 45 and 54 months for recurrence-free and overall survival, respectively. Grade 2-3 sinusoidal dilatation was found in 66 (24.9\%) patients, grade 2-3 steatosis in 30 (11.3\%) patients, and steatohepatitis in one (0.6\%) patient. With hardly observed liver fibrosis, nodular regeneration was present in 41 (19.3\%) of 212 patients with collagen staining available. In multivariable analysis, severe steatosis was related to increased surgical site infections (OR 3.55, 95\% Cl 1.39-9.07, p=0.008) and systemic sepsis (OR 6.73, 95\% Cl 1.34-33.73, $\mathrm{p}=0.020)$. Neither sinusoidal dilatation nor nodular regeneration was related to infectious complications. Presence of deep or organ/space surgical site infections were associated with decreased recurrence-free ( $\mathrm{HR} 1.71,95 \% \mathrm{Cl} 1.04-2.80, p=0.035)$, and overall survival ( $\mathrm{HR} 2.04,95 \% \mathrm{Cl} 1.31-3.19, \mathrm{p}=0.002)$. None of the studied background liver abnormalities was related to survival.

\section{Conclusions}

Chemotherapy-associated hepatic steatosis was associated with increased infectious complications, which were, in turn, related to decreased recurrence-free and overall survival. 


\section{Introduction}

Postoperative infectious complications occur often after partial hepatectomy for colorectal liver metastases. ${ }^{1}$ The presence of infectious complications, particularly surgical site infections, remains a crucial clinical problem and is related to prolonged length of hospital stay, ${ }^{1}$ increased healthcare cost, ${ }^{2,3}$ in-hospital mortality, ${ }^{4}$ and even decreased long-term survival. ${ }^{4,5}$ Although many advances have been made in infection control in the past decades, surgical site infections remain the second most frequent healthcare-related infection after respiratory tract infection. ${ }^{6}$

Neoadjuvant chemotherapy for patients with colorectal liver metastases is used to downstage the tumour and to facilitate future hepatic resection. ${ }^{7,8}$ However, systemic chemotherapy may also cause undesired liver parenchymal damage, referred to as chemotherapy-associated liver injury (CALI). For instance, the combination of 5 -fluorouracil and leucovorin is related to the development of steatosis, ${ }^{9}$ irinotecanbased regimens are associated with increased occurrence of steatohepatitis, ${ }^{9-11}$ and oxaliplatin-based treatments appear to be related to the development of sinusoidal obstruction syndrome ${ }^{12}$ as well as nodular regeneration. ${ }^{13}$

The development of CALI has several, potentially serious adverse downstream effects. It has been shown by our group ${ }^{14}$ and subsequently by others ${ }^{15}$ that CALI is associated with poor tumour response to neoadjuvant chemotherapy. Moreover, CALI is related to increased surgical morbidity ${ }^{16-19}$ and mortality, ${ }^{20}$ and may even reduce survival ${ }^{21}$ of patients with colorectal liver metastases undergoing partial hepatectomy. Unfortunately, our understanding of the impact of CALI on the incidence of postoperative infectious complications after partial hepatectomy is poor. Therefore, the primary aim of the present study was to explore whether CALI (i.e. sinusoidal dilatation, nodular regeneration, steatosis, and steatohepatitis) is associated with increased infectious complications, and, secondly, to investigate if these factors are associated with survival of patients with colorectal liver metastases who underwent partial hepatectomy.

\section{Methods}

\section{Inclusion of patients}

Consecutive patients who had undergone partial hepatectomy for colorectal liver metastases at Maastricht University Medical Centre, a tertiary referral hospital, between January 2008 and December 2013 were considered for this study if there was an 
adequate histopathological assessment of non-tumour bearing liver tissue (i.e. presence of non-tumour-bearing liver at a distance of more than $2 \mathrm{~cm}$ from the tumour). Patients with cirrhosis were excluded from the study.

\section{Definitions and data collection}

Data regarding the postoperative infectious complications were prospectively monitored daily by an independent infection-control nurse (MR) based on the following definitions. Postoperative infectious complications were categorized as a combination of surgical site infections, remote site infections, and systemic sepsis developed within 30 days after surgery or during hospital stay. Surgical site infections were defined according to the guideline of the Centres for Disease Control and Prevention (CDC)'s recommendations, including superficial incisional, deep incisional, and intraabdominal organ/space infections. ${ }^{22}$ Severe surgical site infections were defined as the presence of either deep incisional or intraabdominal organ/space infections. Remote site infections were described as a condition in which fever and leukocytosis were present with bacteria in urine, sputum, and/or central venous catheter tips according to an experienced physician's judgment with consideration of microbiologic evidence when available.

Comorbidity was defined as any disease affecting the patient apart from colorectal liver metastases (i.e. diabetes mellitus, and pulmonary, renal, cardiovascular, and other diseases). Overall morbidity was defined as any complication occurring within 30 days after surgery or during hospital stay and was categorized according to Dindo et al. ${ }^{23}$ Radical resection was defined as resected tumour lesions with a surgical margin of more than one millimeter. All patients were admitted one-day prior to liver surgery, and the length of hospital stay was defined as the duration between the date of admission and the date of discharge.

\section{Pathology assessment of non-tumour-bearing liver}

Sinusoidal dilatation and nodular regeneration were semi-quantitatively assessed by two experienced liver pathologists (AW and CV). Diagnosis of nodular regeneration was made only when confirming liver fibrosis was absent or minor. Assessment of nonalcoholic fatty liver disease was performed by another experienced liver pathologist (JV) independently. All pathologists were blinded to clinical information concerning the patients. 
The resected liver tissue was fixed in formalin, embedded in paraffin, and stained with hematoxylin \& eosin and reticulin. Sinusoidal dilatation was graded according to RubbiaBrandt et al. ${ }^{24}$ Nodular regeneration was graded according to the Wanless scoring system. ${ }^{25}$ Steatosis was graded according to Kleiner et al. ${ }^{26}$ According to the recently established SAF scoring system, a case presenting with at least grade 1 of each of the three features (i.e. steatosis, hepatocellular ballooning, and lobular inflammation) was classified as steatohepatitis. ${ }^{27}$

\section{Statistical analyses}

All statistical analyses were performed using IBM SPSS Statistics for Windows (version 20.0), and all graphs were drawn using GraphPad Prism for Windows (Version 6.05). For multiple comparisons of numerical variables, patient characteristics were compared using One-way ANOVA and Tukey's multiple comparisons test. For categorical variables, the Pearson Chi-square test with continuity correction was applied, or the Fisher's exact test when any of the expected values was smaller than five. The relation between CALI and postoperative infectious complications was studied applying uni- and multivariable binary logistic regression models.

The association between CALI, infectious complications, and survival was analyzed using uni- and multivariable Cox regression models. To specify which type of postoperative morbidities were the key component related to survival, multivariable analysis was performed subsequently in three-steps: step one, overall morbidity vs. absent morbidity; step two, any infectious morbidity vs. non-infectious morbidity vs. absent morbidity; step three, severe surgical site infections vs. remote site infections vs. superficial surgical site infections or sepsis vs. non-infectious morbidity vs. absent morbidity.

Variables with $p$-value $\leq 0.10$ in univariable analysis were included in the multivariable analysis. Odds ratios (OR) or hazard ratios (HR) with $95 \%$ confidence intervals $(\mathrm{Cl})$ were calculated. A p-value $\leq 0.05$ was considered significant for all tests.

\section{Results}

A total of 265 consecutive patients were included (Table 4.1), of which 172 (64.9\%) were male and 93 (35.1\%) female, with a median age of 66 (inter quartile range 59-72) years. 
Table 4.1 General characteristics

\begin{tabular}{|c|c|}
\hline Factors & $n=265$ \\
\hline \multicolumn{2}{|l|}{ General characteristics } \\
\hline Sex (male) & 172 (64.9\%) \\
\hline Age (year) & $66(59-72)$ \\
\hline Body mass index $\left(\mathrm{kg} / \mathrm{m}^{2}\right)$ & $26.0(23.7-28.6)$ \\
\hline Comorbidity & $169(63.8 \%)$ \\
\hline Diabetes & $22(8.3 \%)$ \\
\hline Cardiovascular & $117(44.2 \%)$ \\
\hline Pulmonary & $28(10.6 \%)$ \\
\hline Renal & $8(3.0 \%)$ \\
\hline Others & $71(26.8 \%)$ \\
\hline Portal venous embolization & $12(4.5 \%)$ \\
\hline \multicolumn{2}{|l|}{ Primary colorectal tumour } \\
\hline Rectal cancer & $108(40.8 \%)$ \\
\hline Tumour stage 3-4 ( $\left.n=260^{*}\right)$ & $220(83.0 \%)$ \\
\hline Lymph node with tumour cells ( $n=257^{*}$ ) & $167(63.0 \%)$ \\
\hline \multicolumn{2}{|l|}{ Liver metastases } \\
\hline Synchronous metastases (<12 months) & $183(69.1 \%)$ \\
\hline Number of metastases & $2(1-3)$ \\
\hline Diameter of largest metastases & $2.5(1.5-4.0)$ \\
\hline \multicolumn{2}{|l|}{ Chemotherapy details } \\
\hline Preoperative chemotherapy & $184(69.4 \%)$ \\
\hline 5-fluorouracil & $6(2.3 \%)$ \\
\hline Oxaliplatin & 167 (61.9\%) \\
\hline Capecitabine & $170(64.2 \%)$ \\
\hline Irinotecan & $9(3.4 \%)$ \\
\hline Bevacizumab & $114(43.0 \%)$ \\
\hline \multicolumn{2}{|l|}{ Surgical details } \\
\hline Major hepatectomy ( $\geq 3$ Couinaud segments) & 119 (44.9\%) \\
\hline Surgical duration in hour $\left(n=263^{*}\right)$ & $3.4(2.5-4.5)$ \\
\hline Pringle manoeuver $\left(n=264^{*}\right)$ & $67(25.3 \%)$ \\
\hline Laparoscopic surgery & $24(9.1 \%)$ \\
\hline Perioperative blood transfusion & $48(18.1 \%)$ \\
\hline Radical resection & $184(69.4 \%)$ \\
\hline \multicolumn{2}{|l|}{ Postoperative characteristics } \\
\hline Overall morbidity & $125(47.2 \%)$ \\
\hline Infectious complications & 82 (30.9\%) \\
\hline Surgical site infections & $63(23.8 \%)$ \\
\hline Superficial wound infection & $33(12.5 \%)$ \\
\hline Deep wound infection & $9(3.4 \%)$ \\
\hline Intraabdominal abscess & $16(6.0 \%)$ \\
\hline Liver abscess & $23(8.7 \%)$ \\
\hline Remote site infections & $41(15.5 \%)$ \\
\hline Urinary tract infection & $21(7.9 \%)$ \\
\hline Respiratory infection & $26(9.8 \%)$ \\
\hline Central venous catheter infection & $6(2.3 \%)$ \\
\hline Sepsis & $20(7.5 \%)$ \\
\hline Ninety-day mortality & $11(4.2 \%)$ \\
\hline Length of hospital stay (day) & $8(6-11)$ \\
\hline
\end{tabular}

Numeric variables are presented as median with interquartile range; *Due to missing data, indicated number of patients was below 265 . 
Preoperative comorbidity was present in 169 (63.8\%) patients. Twelve (4.5\%) patients had undergone unilateral portal venous embolization before liver surgery. 148 (55.8\%) patients developed more than one liver metastasis. 184 (69.4\%) patients received preoperative chemotherapy. Capecitabine in combination with oxaliplatin (CAPOX) was the most commonly used regimen, and was administered to 167 (90.8\%) patients. Bevacizumab was co-administered to 114 (62.0\%) patients. Postoperative surgical site infections were present in $63(23.8 \%)$ patients, remote site infections were found in $41(15.5 \%)$ patients, and systemic sepsis was observed in 20 (7.5\%) patients. The median length of hospital stay was 8 (interquartile range 6-11) days, and 247 (93.8\%) patients were discharged within 30 days after admission.

Table 4.2 summarizes the pathology details of chemotherapy-associated liver injury. Of the 148 (55.8\%) patients who presented with sinusoidal dilatation, mild lesions (grade 1 ) were found in $82(30.9 \%)$ subjects, and severe lesions (grade 2 or grade 3 ) were observed in 66 (24.9\%) patients. Mild steatosis (grade 1) was present in 21 (7.9\%) patients, and severe steatosis (grade 2 and grade 3) was found in 30 (11.3\%) patients. Collagen staining, which was required to assess nodular regeneration, was available from 212 patients, and revealed nodular regeneration in $41(15.5 \%)$ of them; severe nodular regeneration (grade 2 or grade 3) was observed in 20 (9.4\%) patients. Steatohepatitis was found in one $(0.4 \%)$ patient, who had grade 2 steatosis ( $40 \%$ steatotic hepatocytes), grade 1 lobular inflammation, and grade 2 hepatocellular ballooning, but absent sinusoidal dilatation.

Table 4.2 Pathology characteristics.

\begin{tabular}{lc}
\hline Pathology details & $\mathrm{n}=265$ \\
\hline Sinusoidal dilatation & \\
Absent & $117(44.2 \%)$ \\
Grade 1 & $82(30.9 \%)$ \\
Grade 2 & $43(16.2 \%)$ \\
Grade 3 & $23(8.7 \%)$ \\
Nodular regeneration $\left(\mathrm{n}=212^{*}\right)$ & \\
Absent & $171(80.7 \%)$ \\
Grade 1 & $21(7.9 \%)$ \\
Grade 2 & $13(4.9 \%)$ \\
Grade 3 & $7(2.6 \%)$ \\
Steatosis & \\
Absent & $109(41.1 \%)$ \\
Grade 1 & $126(47.5 \%)$ \\
Grade 2 & $22(8.3 \%)$ \\
Grade 3 & $8(3.0 \%)$ \\
Steatohepatitis & $1(0.4 \%)$ \\
\hline
\end{tabular}

*Due to unavailable collagen staining, indicated number of patients was below 265. 
The presence of severe sinusoidal dilatation was higher in patients who presented with nodular regeneration than in those without these lesions ( $n=212,28 / 41$ vs. 31/171, $\mathrm{p}<0.001)$. In contrast, the incidence of severe sinusoidal dilatation in patients with severe steatosis was lower than that of non-severe lesions ( $1 / 30$ vs. 65/235, $p=0.002$ ). Subgroup multivariable analysis (Supplemental Table S4.1) showed that both grade 1 (OR 0.32, 95\% Cl 0.12-0.86, $\mathrm{p}=0.024$ ) and grade 2-3 sinusoidal dilatation (OR 0.05, 95\% $\mathrm{Cl} 0.01-0.43, p=0.006$ ) were inversely related to the occurrence of severe steatosis. Both irinotecan-based treatment (OR 9.66, 95\% Cl 1.42-65.49, $\mathrm{p}=0.020)$ and body mass index (OR 1.27, 95\% Cl 1.13-1.42, p<0.001) were independently associated with increased severe steatosis.

Multivariable analysis showed that perioperative blood transfusion (OR 2.14, 95\% Cl 1.09-4.22, $p=0.027$ ) was the sole factor significantly related to increased infectious complications. Surgical duration (OR 1.14, 95\% Cl 0.98-1.33, $\mathrm{p}=0.098$ ) and severe steatosis (OR 2.33, 95\% Cl 1.97-5.59, $\mathrm{p}=0.059$ ) tended to be associated with increased infectious complications. To clarify which type of infectious complications (e.g. surgical site infections, remote site infections, and systemic sepsis) was associated with background liver abnormalities, further multivariable analyses were performed (Figure 4.1, detailed data are summarized in supplemental Table S4.2).
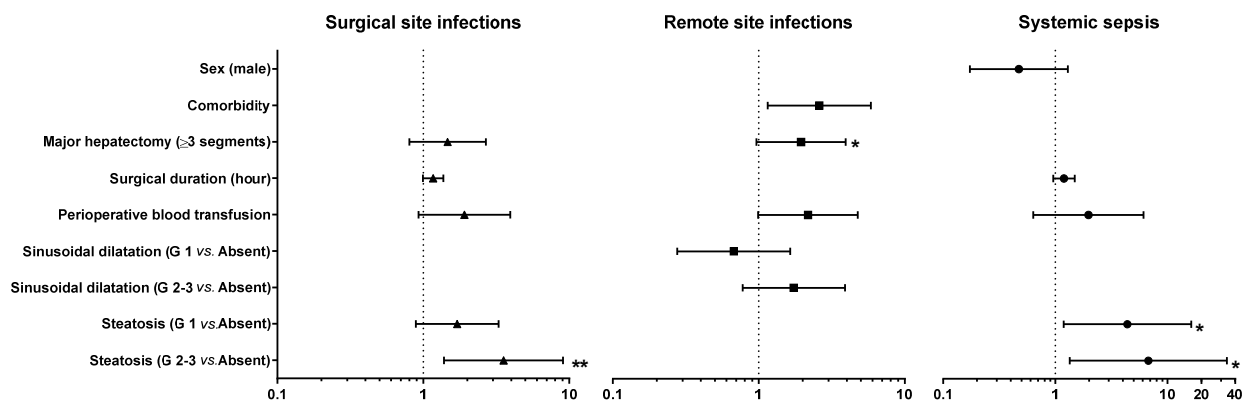

Figure 4.1 Factors related to postoperative infectious complications. Data are depicted as odds ratios (symbols) with 95\% confidence intervals (bars) based on results of multivariable analysis, variable with $\mathrm{p} \leq 0.10$ in univariable analysis were induced in the multivariable analysis, one asterisk indicates $p$-value $\leq 0.05$, two asterisks indicate $p$-value $\leq 0.01$.

Severe steatosis was associated with increased incidence of surgical site infections (OR 3.55, 95\% Cl 1.39-9.07, $\mathrm{p}=0.008$ ). Both grade 1 (OR 4.38, 95\% Cl 1.18-16.21) and grade 2-3 steatosis (OR $6.73,95 \% \mathrm{Cl} 1.34-33.73, p=0.020$ ) were related to systemic sepsis. Sinusoidal dilatation and nodular regeneration were not related to any type of infectious complications. The presence of comorbidity was the sole factor significantly related to 
remote site infections ( $O R 2.59,95 \% \mathrm{Cl}$ 1.15-5.84, $\mathrm{p}=0.022$ ). To investigate whether steatosis was related to superficial and/or severe surgical site infections, a subgroup analysis was performed (supplemental Table S4.3). It was shown that severe steatosis was only related to increased superficial surgical site infections (OR 4.50, 95\% Cl 1.4314.10, $p=0.010$ ), but not to severe surgical site infections (OR 1.14, 95\% Cl 0.34-3.78, $\mathrm{p}=0.835)$. Both major hepatectomy (OR 2.31, 95\% Cl 1.10-4.84, $\mathrm{p}=0.027)$ and surgical duration (OR 1.20, 95\% Cl 1.01-1.42, $\mathrm{p}=0.037$ ) were associated with increased severe surgical site infections.

The relationship between potential prognostic factors and recurrence-free survival is summarized in Figure 4.2 (detailed data are summarized in supplemental Table S4.4). The median period of follow-up for recurrence-free survival was 45 months. None of the studied background liver abnormalities was related to recurrence-free survival. In multivariable analysis, the presence of severe surgical site infections was the sole factor related to a 71 percent increased hazard of tumour recurrence (HR 1.71, 95\% Cl 1.042.80, $\mathrm{p}=0.035)$. Moreover, remote site infections tended to be associated with recurrence-free survival ( $\mathrm{HR} 1.68,95 \% \mathrm{Cl} 0.91-3.08, \mathrm{p}=0.096)$. Both radical resection (HR $0.54,95 \% \mathrm{Cl} 0.37-0.79, \mathrm{p}<0.001)$ and body mass index (HR 0.95, 95\% Cl 0.91-0.99, $\mathrm{p}=0.015$ ) were associated with decreased risk of tumour recurrence.

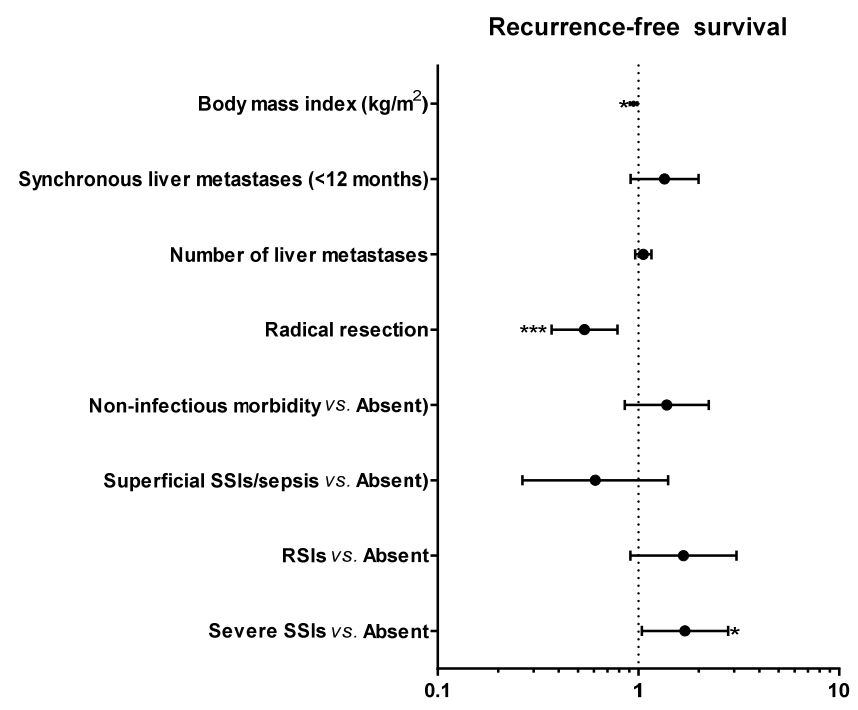

Figure 4.2 Prognostic factors related to recurrence-free survival. Data are depicted as hazard ratios (symbols) with 95\% confidence intervals (bars) based on results of multivariable analysis; one asterisk indicates $p$-value $\leq 0.05$, two asterisks indicate $p$-value $\leq 0.01$, and three asterisks indicate p-value $\leq 0.001$; SSIs, surgical site infections; RSIs, remote site infections. 
Figure 4.3 summarizes the association between potential prognostic factors and overall survival (detailed data are summarized in supplemental Table S4.5). Median period of follow-up for overall survival was 54 months. Again, none of the studied background liver abnormalities was associated with overall survival. Severe surgical site infections were related to a 104 percent increased hazard of mortality (HR 2.04, 95\% Cl 1.31-3.19, $\mathrm{p}=0.002)$. Remote site infections ( $H R$ 1.22, 95\% Cl 0.71-2.12, $\mathrm{p}=0.474)$, superficial surgical site infections or sepsis (HR 1.35, 95\% Cl 0.69-2.66, $\mathrm{p}=0.379$ ), and non-infectious complications ( $\mathrm{HR} 0.83,95 \% \mathrm{Cl} 0.50-1.37, \mathrm{p}=0.466$ ) were not associated with overall survival. Age (HR 1.02, 95\% Cl 1.00-1.04, $\mathrm{p}=0.025)$, synchronous liver metastases (HR $1.51,95 \% \mathrm{Cl} 1.03-2.20, \mathrm{p}=0.033)$, and the number of liver metastases ( $\mathrm{HR} 1.11,95 \% \mathrm{Cl}$ 1.03-1.19, $\mathrm{p}=0.005)$ were related to decreased overall survival. Conversely, radical resection ( $\mathrm{HR} 0.52,95 \% \mathrm{Cl} 0.37-0.74, \mathrm{p}<0.001)$ and major hepatectomy $(\mathrm{HR} 0.64,95 \% \mathrm{Cl}$ $0.44-0.93, p=0.018$ ) were associated with increased overall survival.

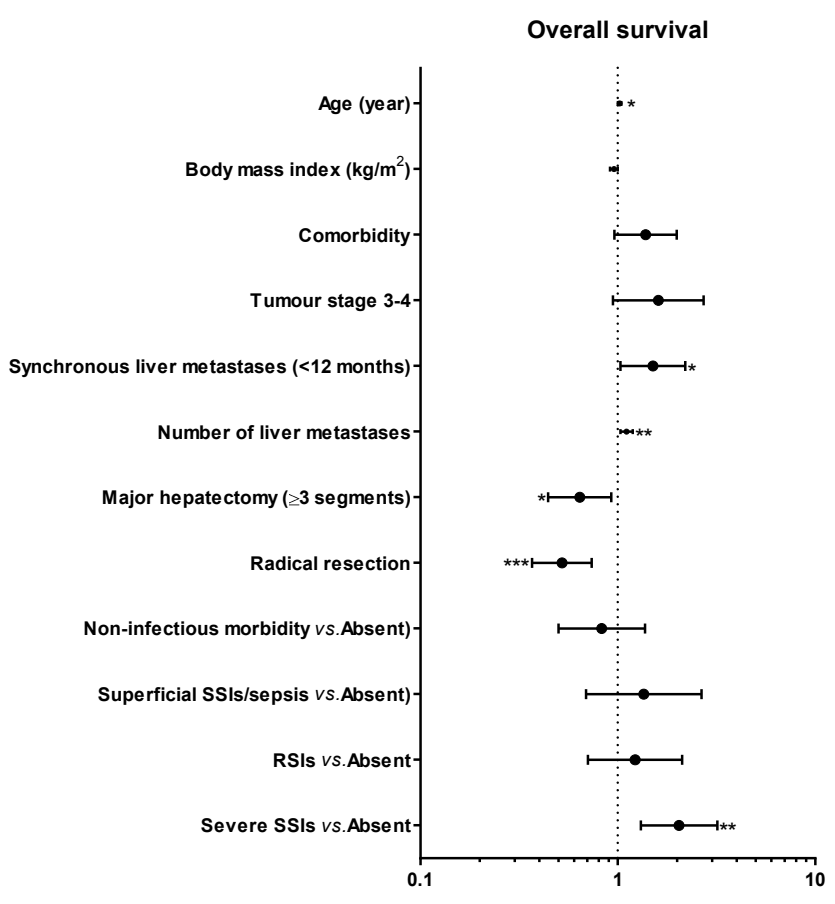

Figure 4.3 Prognostic factors related to overall survival. Data are depicted as hazard ratios (symbols) with 95\% confidence intervals (bars) based on results of multivariable analysis; one asterisk indicates $p$-value $\leq 0.05$, two asterisks indicate $p$-value $\leq 0.01$, and three asterisks indicate $p$-value $\leq 0.001$; SSIs, surgical site infections; RSIs, remote site infections. 
Next, we also explored the association between postoperative morbidity, 90-day mortality, and length of hospital stay (Figure 4.4). 90-day mortality occurred more often in patients with severe surgical site infections than in those without (6/39 vs. 3/226, $\mathrm{p}<0.001$ ). Of the occurred six deaths in the group of patients with severe surgical site infections, four patients had liver abscesses, accompanied with one deep wound infection and one intra-abdominal abscess; one patient had only deep wound infection, and another one had intra-abdominal abscess. The mean length of hospital stay for patients with severe surgical site infections was $26.3 \pm 4.3$ days. This was considerably longer than length of stay of patients without morbidity (mean difference 19.3 days, 95\% $\mathrm{Cl} 13.1-25.4, \mathrm{p}<0.001$ ), with non-infectious complications (mean difference 14.3 days, $95 \% \mathrm{Cl}$ 6.8-21.8, $\mathrm{p}<0.001$ ), with superficial surgical site infections/sepsis (mean difference 14.0 days, $95 \% \mathrm{Cl} 4.9-23.2, \mathrm{p}<0.001$ ), and with remote site infections (mean difference 13.6 days, $95 \% \mathrm{Cl} 4.6-22.6, \mathrm{p}<0.001)$.

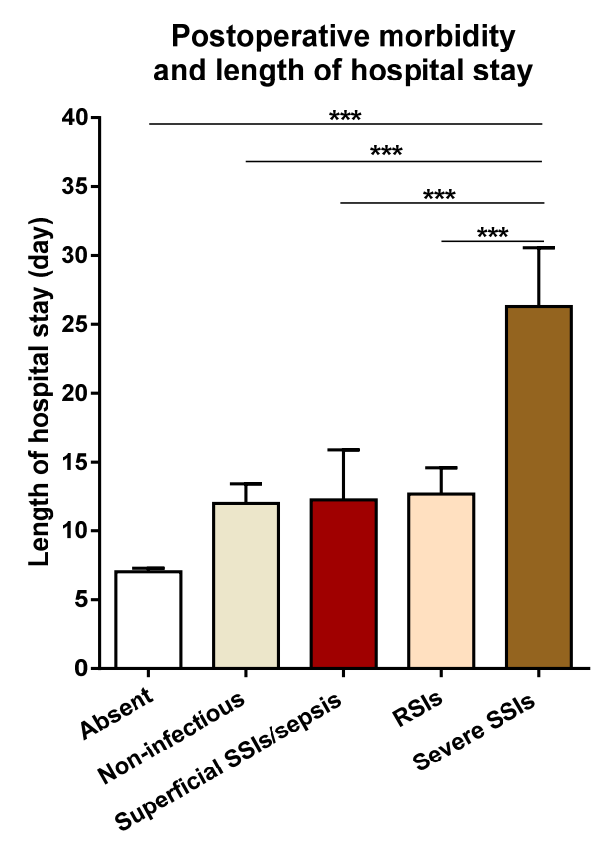

Figure 4.4 Postoperative morbidity and length of hospital stay. One way ANOVA, $p<0.001$; Turkey's multiple comparisons test: $* * *$ indicates $p$-value $<0.001$; Error bars represent means with standard error of means; RSIs, remote site infections; SSIs, surgical site infections. 


\section{Discussion}

In the present study, surgical site infections and systemic sepsis after partial hepatectomy for colorectal liver metastases were found to occur more often in patients with hepatic steatosis, whereas neither sinusoidal dilatation nor nodular regeneration were associated with these infectious complications. Steatosis was specifically related to increased superficial surgical site infections. The presence of severe surgical site infections was associated with decreased long-term recurrence-free survival, and overall survival. None of the studied background liver abnormalities was related to survival. Contrasting to the observed inverse relation between severe steatosis and sinusoidal dilatation, severe sinusoidal dilatation was positively related to the occurrence of nodular regeneration. Irinotecan-based treatment and body mass index were also related to increased severe steatosis.

The fact that severe steatosis was independently associated with the occurrence of surgical site infections is in line with a previous report, ${ }^{28}$ and might be related to the body mass index of patients. As confirmed in the current cohort, an increased body mass index is a risk factor for developing steatosis. ${ }^{29}$ A higher BMI may increase the extent of subcutaneous fat accumulation, which may cause increased tension on surgical sutures, leading to slower wound healing and increased susceptibility to infection. Furthermore, a change in gut microbiota composition seen in obese individuals are known to be associated with hepatic insulin resistance, an important cause of hepatic steatosis, ${ }^{30}$ and with bacterial translocation, ${ }^{31}$ which may further contribute to increased susceptibility to systemic sepsis. Hepatic steatosis may additionally diminish the ability of Kupffer cells to clear translocated bacteria. ${ }^{32}$ In addition, excessive adipose tissue accumulation in obese individuals promotes dysregulated expression of adipokines like leptin, visfatin, adipsin, and angiopoietin-like protein 2 that affect the immune system, potentially increasing susceptibility to infections. ${ }^{33}$

Next to the link between hepatic steatosis and surgical site infections, this study showed that infectious complications, rather than non-infectious events, were related to decreased survival after partial hepatectomy for colorectal liver metastases. In particular, the presence of severe surgical site infections was related to a significantly increased risk of tumour recurrence and long-term mortality, consistent with a previous report ${ }^{5}$. Interestingly, it has been shown by Akhter et al. that surgical site infections occur more often in patients with immune suppression. ${ }^{34}$ In accordance with this, enhancing the immune system may suppress tumour progression, ${ }^{35}$ enhance tumour response, and prolong long-term survival. ${ }^{36}$ In essence, the presence of severe surgical 
site infections may mirror a relatively weaker innate or adaptive immunity. Tumour suppression may therefore be weakened, eventually resulting in more frequent tumour recurrence and decreased long-term overall survival.

Although Tamandl et al. showed a correlation between chemotherapy-associated sinusoidal dilatation and long-term survival after partial hepatectomy for colorectal liver metastases, ${ }^{21}$ neither sinusoidal dilatation nor any other type of liver injury was related to survival in the current study. In the previous cohort, ${ }^{21}$ only patients with preoperative neoadjuvant chemotherapy were included, minimizing the influence of factors other than preoperative chemotherapeutic treatment for developing background liver abnormalities. More importantly, the effect of neoadjuvant chemotherapy on the tumour, expressed as tumour response which is shown to be positively related to survival, ${ }^{15}$ whereas negatively related to the presence of chemotherapy-associated liver injury, ${ }^{14,15}$ may be taken into account potentially. Therefore, future study is needed to determine whether CALI itself or an impaired tumour response as a result of CALI are associated with long-term recurrence-free survival and overall survival after partial hepatectomy for colorectal liver metastases. Because postoperative infectious complications were important prognostic factors for survival in the present study, such factors should be included as confounders in future studies.

Remarkably, in the present study, only one patient was diagnosed with steatohepatitis. This could be explained by the fact that few patients were treated with irinotecan, a known risk factor for steatohepatitis. ${ }^{9-11}$ Alternatively, it could also be due to the fact that steatohepatitis was diagnosed applying the novel SAF scoring system which is more strict as compared to the previously used NAS system. ${ }^{26}$

Because chemotherapy was frequently administered at local referral hospitals, data on the interval between cessation of preoperative chemotherapy and partial hepatectomy, as well as the number of administered cycles, were not available for the majority of studied patients. Therefore, the potential effect of these factors on postoperative infectious complications and survival could not be studied. However, the majority of patients treated with chemotherapy received the same regimen (capecitabine in combination with oxaliplatin). Therefore, the influence of these factors on infectious complications and survival was presumably minimal. Finally, whereas the current study featured a single-centre, retrospective study design, it must be highlighted that data regarding postoperative infectious complications were prospectively collected daily by an experienced independent infection-control nurse. 
In conclusion, this study tried to explore the relationship between two important issues in hepatic surgery (i.e. chemotherapy-associated liver injury and postoperative infectious complications), and their association with survival after partial hepatectomy for colorectal liver metastases. We demonstrated that postoperative surgical site infections and systemic sepsis occurred more often in patients with hepatic steatosis. In particular, the presence of steatosis was related to increased superficial surgical site infections, instead of severe surgical site infections. Severe surgical site infections were associated with increased 90-day mortality and length of hospital stay, and decreased recurrencefree and overall survival. It seems thus reasonable to advise clinicians to adopt preventive strategies concerning infection control when hepatic steatosis is observed through preoperative ultrasound or radiological images, or even if a "yellow liver" is seen during surgery. 


\section{References}

1. Pessaux $P$, van den Broek MA, Wu T, Olde Damink SW, Piardi T, Dejong $\mathrm{CH}$, et al. Identification and validation of risk factors for postoperative infectious complications following hepatectomy. J Gastrointest Surg 2013; 17(11):1907-16.

2. Jenks PJ, Laurent M, McQuarry S, Watkins R. Clinical and economic burden of surgical site infection (SSI) and predicted financial consequences of elimination of SSI from an English hospital. J Hosp Infect 2014; 86(1):24-33.

3. Perencevich EN, Sands KE, Cosgrove SE, Guadagnoli E, Meara E, Platt R. Health and economic impact of surgical site infections diagnosed after hospital discharge. Emerg Infect Dis 2003; 9(2):196-203.

4. Khuri SF, Henderson WG, DePalma RG, Mosca C, Healey NA, Kumbhani DJ, et al. Determinants of longterm survival after major surgery and the adverse effect of postoperative complications. Ann Surg 2005; 242(3):326-41; discussion 341-3.

5. Artinyan A, Orcutt ST, Anaya DA, Richardson P, Chen GJ, Berger DH. Infectious postoperative complications decrease long-term survival in patients undergoing curative surgery for colorectal cancer: a study of 12,075 patients. Ann Surg 2015; 261(3):497-505.

6. Ploegmakers IB, Olde Damink SW, Breukink SO. Alternatives to antibiotics for prevention of surgical infection. Br J Surg 2017; 104(2):e24-e33.

7. Lam VW, Spiro C, Laurence JM, Johnston E, Hollands MJ, Pleass HC, et al. A systematic review of clinical response and survival outcomes of downsizing systemic chemotherapy and rescue liver surgery in patients with initially unresectable colorectal liver metastases. Ann Surg Oncol 2012; 19(4):1292-301.

8. Adam R, Delvart V, Pascal G, Valeanu A, Castaing D, Azoulay D, et al. Rescue surgery for unresectable colorectal liver metastases downstaged by chemotherapy: a model to predict long-term survival. Ann Surg 2004; 240(4):644-57; discussion 657-8.

9. Khan AZ, Morris-Stiff G, Makuuchi M. Patterns of chemotherapy-induced hepatic injury and their implications for patients undergoing liver resection for colorectal liver metastases. J Hepatobiliary Pancreat Surg 2009; 16(2):137-44.

10. Morris-Stiff G, Tan YM, Vauthey JN. Hepatic complications following preoperative chemotherapy with oxaliplatin or irinotecan for hepatic colorectal metastases. Eur J Surg Oncol 2008; 34(6):609-14.

11. Robinson SM, Wilson $\mathrm{CH}$, Burt AD, Manas DM, White SA. Chemotherapy-associated liver injury in patients with colorectal liver metastases: a systematic review and meta-analysis. Ann Surg Oncol 2012; 19(13):4287-99.

12. Rubbia-Brandt L, Lauwers GY, Wang H, Majno PE, Tanabe K, Zhu AX, et al. Sinusoidal obstruction syndrome and nodular regenerative hyperplasia are frequent oxaliplatin-associated liver lesions and partially prevented by bevacizumab in patients with hepatic colorectal metastasis. Histopathology 2010; 56(4):430-9.

13. Vigano L, Rubbia-Brandt L, De Rosa G, Majno P, Langella S, Toso C, et al. Nodular Regenerative Hyperplasia in Patients Undergoing Liver Resection for Colorectal Metastases After Chemotherapy: Risk Factors, Preoperative Assessment and Clinical Impact. Ann Surg Oncol 2015; 22(13):4149-57.

14. Vreuls CP, Van Den Broek MA, Winstanley A, Koek GH, Wisse E, Dejong CH, et al. Hepatic sinusoidal obstruction syndrome (SOS) reduces the effect of oxaliplatin in colorectal liver metastases. Histopathology 2012; 61(2):314-8.

15. Vigano L, Capussotti L, De Rosa G, De Saussure WO, Mentha G, Rubbia-Brandt L. Liver resection for colorectal metastases after chemotherapy: impact of chemotherapy-related liver injuries, pathological tumor response, and micrometastases on long-term survival. Ann Surg 2013; 258(5):731-40; discussion $741-2$.

16. Zorzi D, Laurent A, Pawlik TM, Lauwers GY, Vauthey JN, Abdalla EK. Chemotherapy-associated hepatotoxicity and surgery for colorectal liver metastases. Br J Surg 2007; 94(3):274-86.

17. Nakano H, Oussoultzoglou E, Rosso E, Casnedi S, Chenard-Neu MP, Dufour P, et al. Sinusoidal injury increases morbidity after major hepatectomy in patients with colorectal liver metastases receiving preoperative chemotherapy. Ann Surg 2008; 247(1):118-24.

18. Vigano L, Ravarino N, Ferrero A, Motta M, Torchio B, Capussotti L. Prospective evaluation of accuracy of liver biopsy findings in the identification of chemotherapy-associated liver injuries. Arch Surg 2012; 147(12):1085-91. 
19. Pilgrim CH, Satgunaseelan L, Pham A, Murray W, Link E, Smith M, et al. Correlations between histopathological diagnosis of chemotherapy-induced hepatic injury, clinical features, and perioperative morbidity. HPB (Oxford) 2012; 14(5):333-40.

20. Vauthey JN, Pawlik TM, Ribero D, Wu TT, Zorzi D, Hoff PM, et al. Chemotherapy regimen predicts steatohepatitis and an increase in 90-day mortality after surgery for hepatic colorectal metastases. J Clin Oncol 2006; 24(13):2065-72.

21. TamandI D, Klinger M, Eipeldauer S, Herberger B, Kaczirek K, Gruenberger B, et al. Sinusoidal obstruction syndrome impairs long-term outcome of colorectal liver metastases treated with resection after neoadjuvant chemotherapy. Ann Surg Oncol 2011; 18(2):421-30.

22. Mangram AJ, Horan TC, Pearson ML, Silver LC, Jarvis WR. Guideline for Prevention of Surgical Site Infection, 1999. Centers for Disease Control and Prevention (CDC) Hospital Infection Control Practices Advisory Committee. Am J Infect Control 1999; 27(2):97-132; quiz 133-4; discussion 96.

23. Dindo D, Demartines N, Clavien PA. Classification of surgical complications: a new proposal with evaluation in a cohort of 6336 patients and results of a survey. Ann Surg 2004; 240(2):205-13.

24. Rubbia-Brandt L, Audard V, Sartoretti $P$, Roth AD, Brezault $C$, Le Charpentier $M$, et al. Severe hepatic sinusoidal obstruction associated with oxaliplatin-based chemotherapy in patients with metastatic colorectal cancer. Ann Oncol 2004; 15(3):460-6.

25. Wanless IR. Micronodular transformation (nodular regenerative hyperplasia) of the liver: a report of 64 cases among 2,500 autopsies and a new classification of benign hepatocellular nodules. Hepatology 1990; 11(5):787-97.

26. Kleiner DE, Brunt EM, Van Natta M, Behling C, Contos MJ, Cummings OW, et al. Design and validation of a histological scoring system for nonalcoholic fatty liver disease. Hepatology 2005; 41(6):1313-21.

27. Bedossa P, Consortium FP. Utility and appropriateness of the fatty liver inhibition of progression (FLIP) algorithm and steatosis, activity, and fibrosis (SAF) score in the evaluation of biopsies of nonalcoholic fatty liver disease. Hepatology 2014; 60(2):565-75.

28. Kurmann A, Wanner B, Martens F, Klasen J, Stickel F, Montani M, et al. Hepatic steatosis is associated with surgical-site infection after hepatic and colorectal surgery. Surgery 2014; 156(1):109-16.

29. Zhao J, van Mierlo KMC, Gomez-Ramirez J, Kim H, Pilgrim CHC, Pessaux P, et al. Systematic review of the influence of chemotherapy-associated liver injury on outcome after partial hepatectomy for colorectal liver metastases. Br J Surg 2017;104(8):990-1002.

30. Konrad D, Wueest S. The gut-adipose-liver axis in the metabolic syndrome. Physiology (Bethesda) 2014; 29(5):304-13.

31. Vrakas S, Mountzouris KC, Michalopoulos G, Karamanolis G, Papatheodoridis G, Tzathas C, et al. Intestinal Bacteria Composition and Translocation of Bacteria in Inflammatory Bowel Disease. PLoS One 2017; 12(1):e0170034.

32. Jenne CN, Kubes P. Immune surveillance by the liver. Nat Immunol 2013; 14(10):996-1006.

33. Carbone F, La Rocca C, De Candia P, Procaccini C, Colamatteo A, Micillo T, et al. Metabolic control of immune tolerance in health and autoimmunity. Semin Immunol 2016; 28(5):491-504.

34. Akhter MS, Verma R, Madhukar KP, Vaishampayan AR, Unadkat PC. Incidence of surgical site infection in postoperative patients at a tertiary care centre in India. J Wound Care 2016; 25(4):210-2, 214-7.

35. Shrivastava P, Singh SM, Singh N. Effect of thymosin alpha 1 on the antitumor activity of tumorassociated macrophage-derived dendritic cells. J Biomed Sci 2004; 11(5):623-30.

36. Lipson SD, Chretien PB, Makuch R, Kenady DE, Cohen MH. Thymosin immunotherapy in patients with small cell carcinoma of the lung: correlation of in vitro studies with clinical course. Cancer 1979; 43(3):863-70. 


\section{Supplemental tables}

Table S4.1 Factors related to hepatic steatosis.

\begin{tabular}{|c|c|c|c|c|}
\hline \multirow[b]{3}{*}{ Factors } & \multicolumn{4}{|c|}{ Severe steatosis } \\
\hline & \multicolumn{2}{|c|}{ univariable } & \multicolumn{2}{|c|}{ multivariable } \\
\hline & OR $(95 \% \mathrm{Cl})$ & $\mathrm{p}$-value & OR $(95 \% \mathrm{Cl})$ & $\mathrm{p}$-value \\
\hline Sex (male) & $0.93(0.42-2.04)$ & 0.848 & - & - \\
\hline Age (year) & $0.99(0.96-1.03)$ & 0.706 & - & - \\
\hline Body mass index $\left(\mathrm{kg} / \mathrm{m}^{2}\right)$ & $1.25(1.12-1.39)$ & $<0.001$ & $1.27(1.13-1.42)$ & $<0.001$ \\
\hline Comorbidity & $0.71(0.33-1.54)$ & 0.391 & - & - \\
\hline Portal venous embolization & $0.70(0.09-5.64)$ & 0.739 & - & - \\
\hline 5-fluorouracil & $1.59(0.18-14.05)$ & 0.679 & - & - \\
\hline Oxaliplatin & $1.26(0.57-2.82)$ & 0.568 & - & - \\
\hline Capecitabine & $1.35(0.59-3.07)$ & 0.479 & - & - \\
\hline Irinotecan ${ }^{\#}$ & $2.33(0.46-11.75)$ & 0.307 & $9.66(1.42-65.49)$ & 0.020 \\
\hline Addition of bevacizumab & $1.86(0.86-4.01)$ & 0.113 & - & - \\
\hline \multicolumn{5}{|l|}{ Sinusoidal dilatation } \\
\hline absent & 1 & & 1 & \\
\hline Grade 1 & $0.40(0.16-0.99)$ & 0.049 & $0.32(0.12-0.86)$ & 0.024 \\
\hline Grade $2-3$ & $0.07(0.01-0.51)$ & 0.009 & $0.05(0.01-0.43)$ & 0.006 \\
\hline \multicolumn{5}{|l|}{ Nodular regeneration } \\
\hline Absent & 1 & & & \\
\hline Grade 1 & $0.36(0.05-2.80)$ & 0.327 & - & - \\
\hline Grade $2-3 *$ & N./A. & N./A. & - & - \\
\hline
\end{tabular}

\# As reported as risk factor for steatosis in literature, irinotecan was forced into multivariable model; * the proportion of patients with grade 2-3 nodular regeneration is $100 \%$ for patients without severe steatosis and $0 \%$ for those with severe steatosis (Fisher's exact test: $p=0.200$ ); N./A., not applicable. 


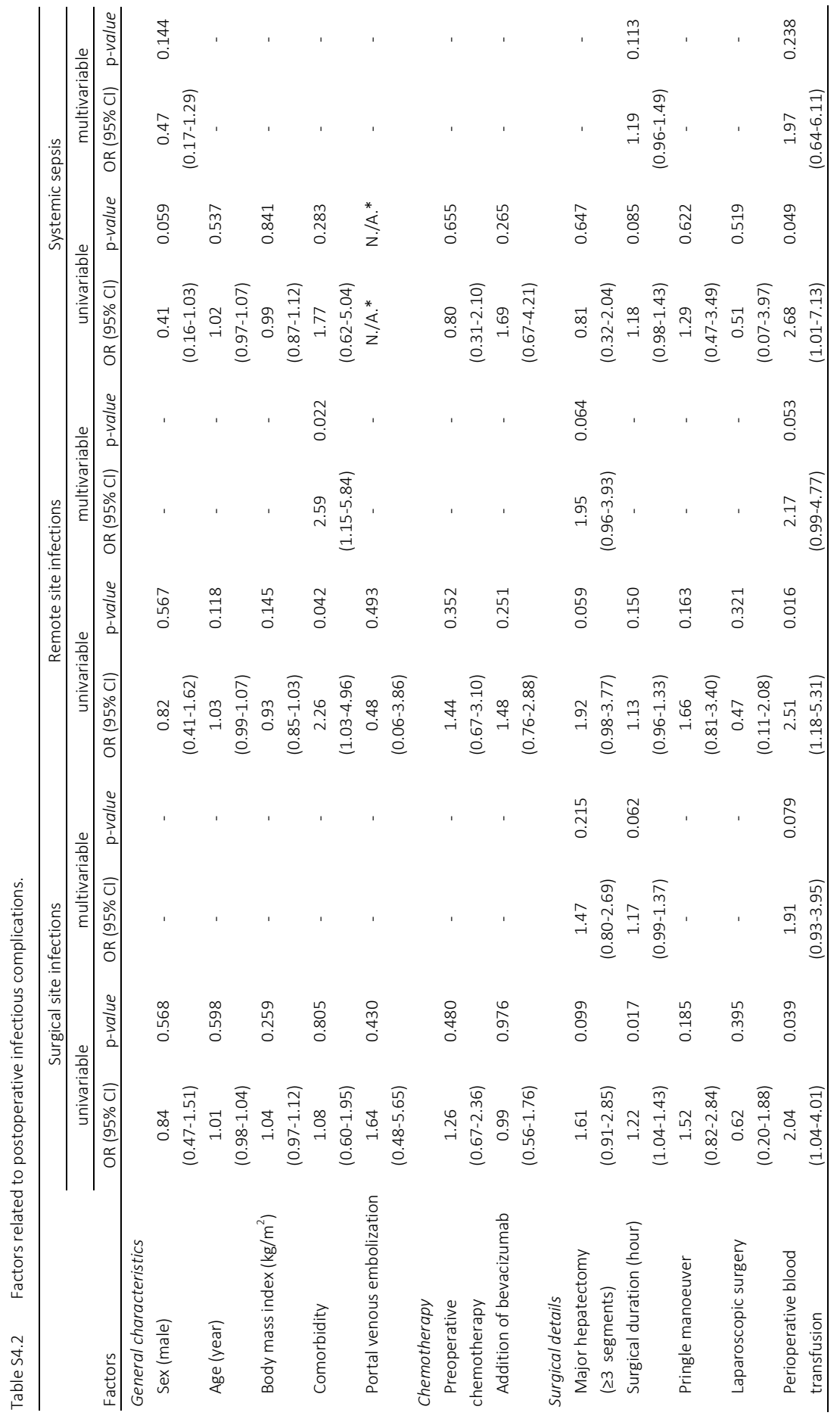




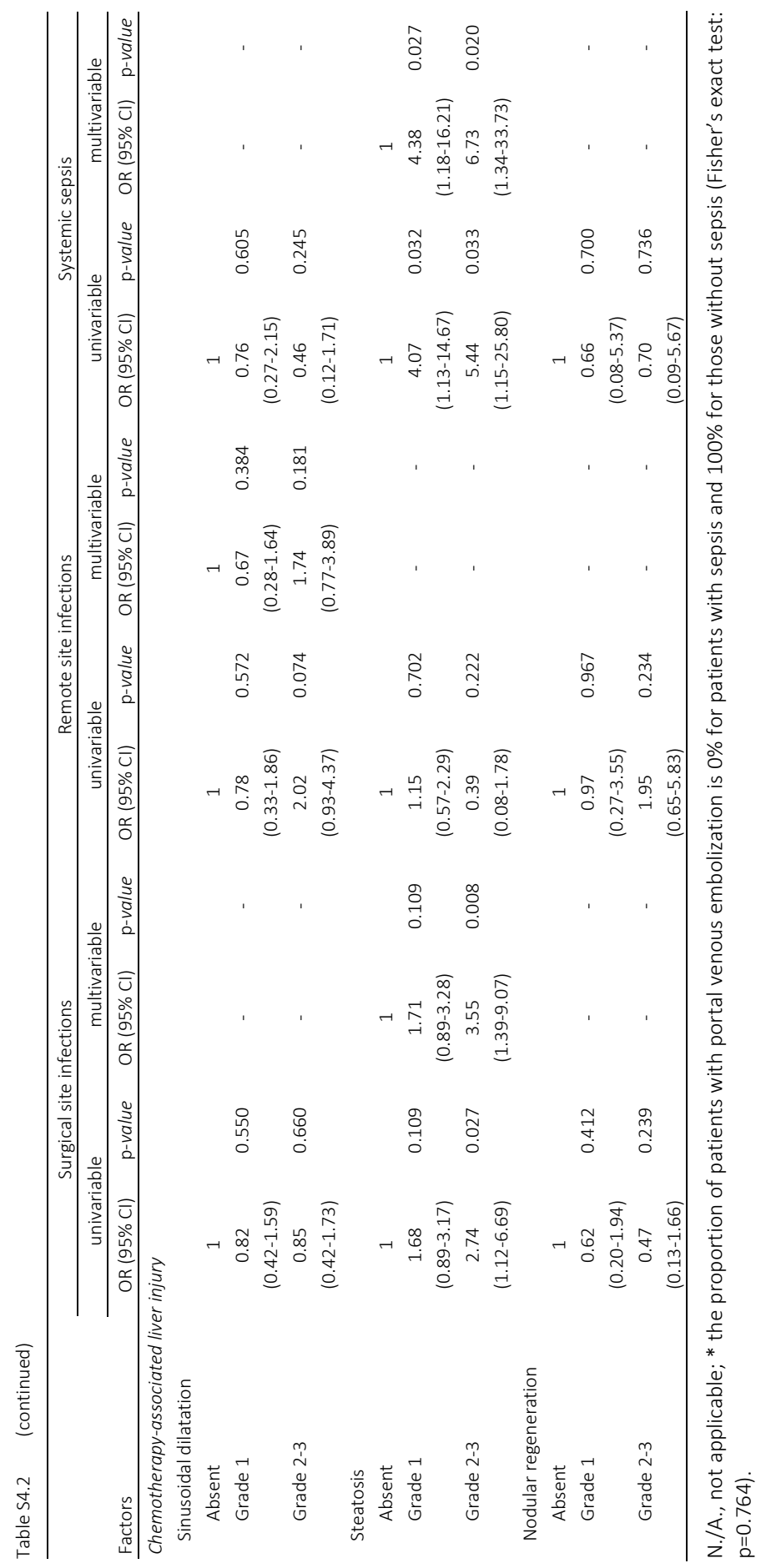




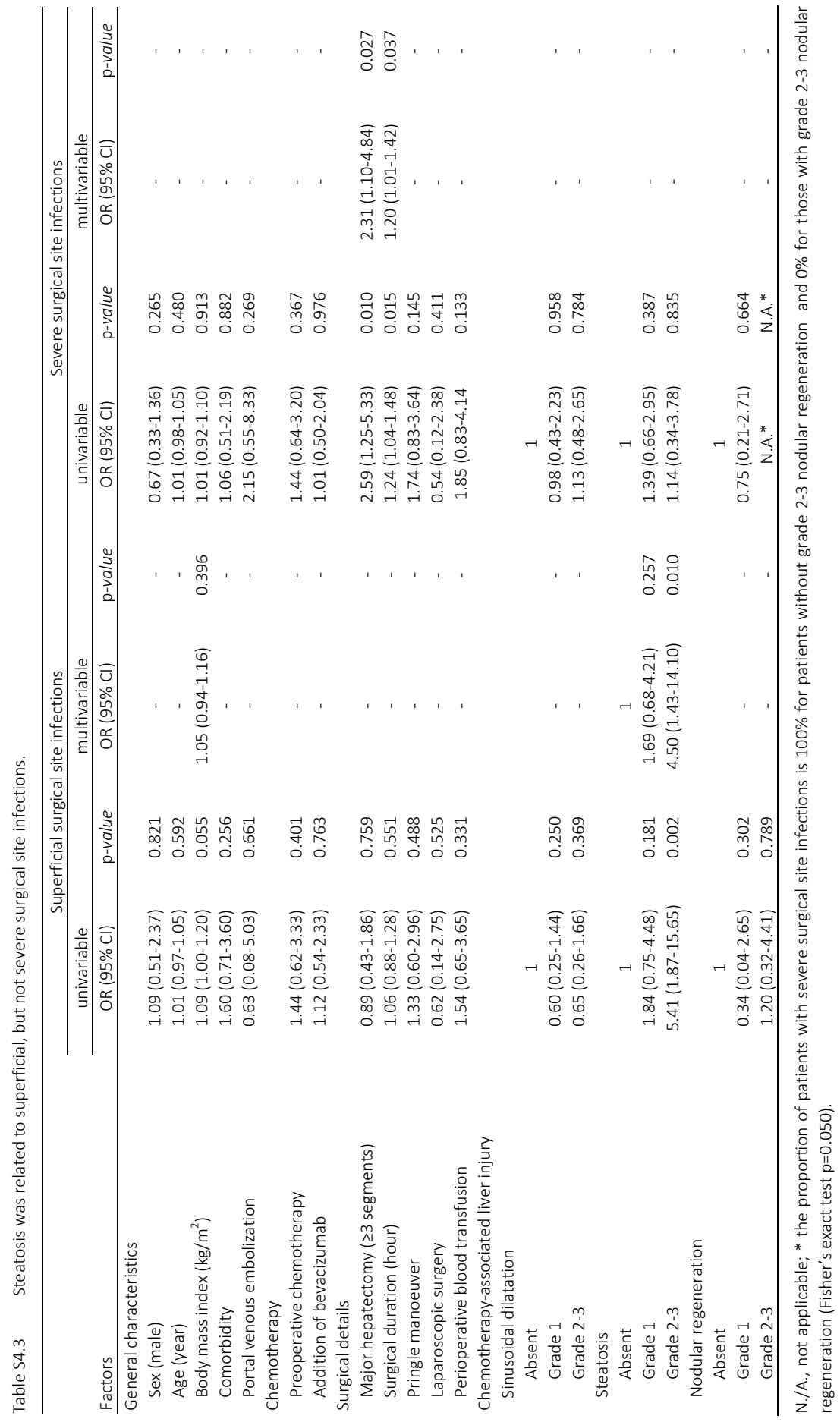




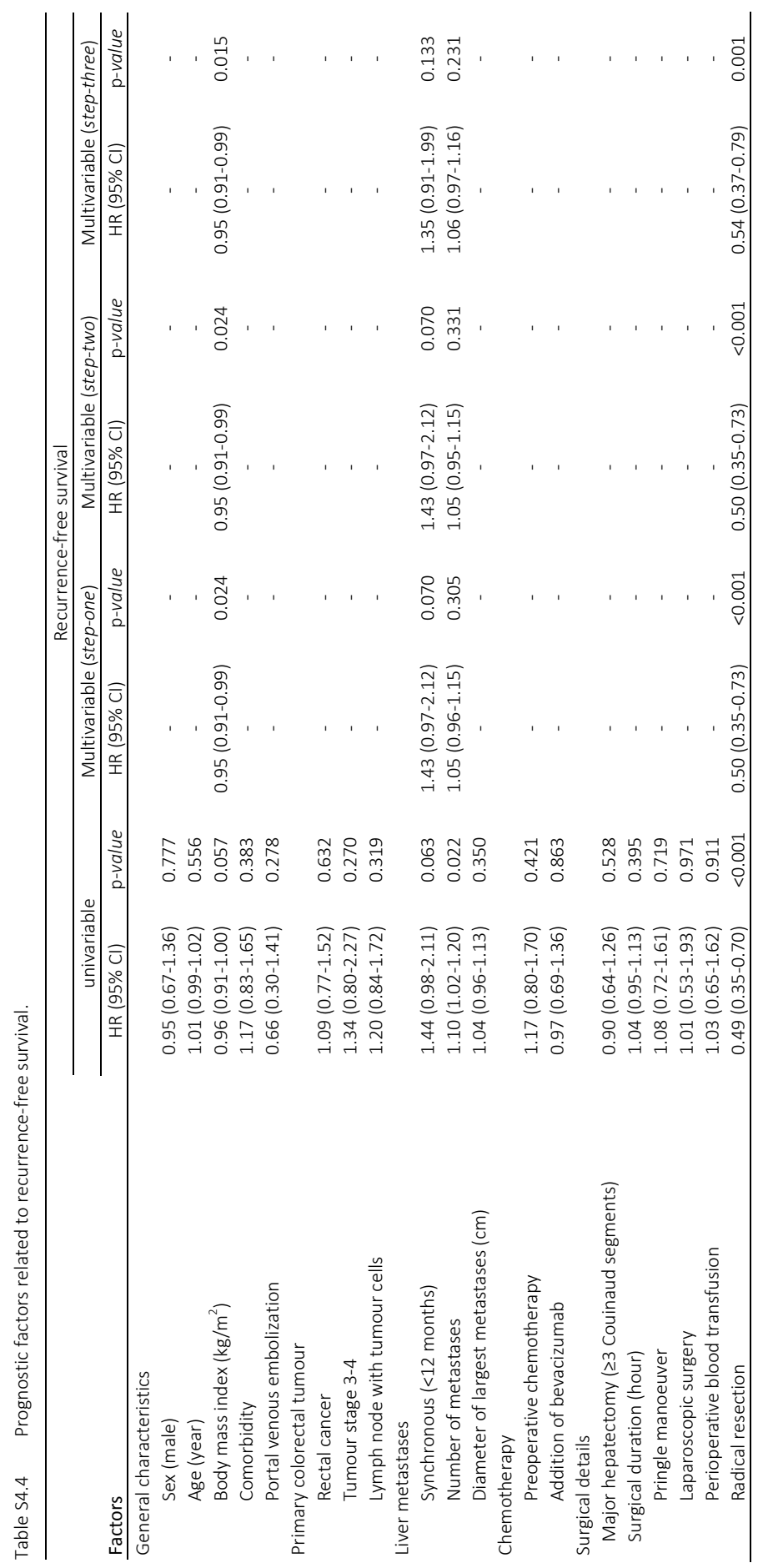




\title{
Chapter
}

\begin{abstract}
Impact of chemotherapy-associated liver injury on tumour regression grade and survival after partial hepatectomy for colorectal liver metastases
\end{abstract}

Junfang Zhao, Pamir Sawo, Sander S. Rensen, Magriet M.J. Rouflart, Alison Winstanley, Celien P.H. Vreuls, Joanne Verheij, Kim M.C. van Mierlo, Toine M. Lodewick, Victor van Woerden, Frank H. van Tiel, Ronald M. van Dam, Cornelis H.C. Dejong, Steven W.M. Olde Damink Submitted 


\section{Abstract}

Introduction

An inverse relation between chemotherapy-associated liver injury (CALI) and tumour response to chemotherapy has been reported in literature. We aimed to validate these findings, and further investigate the impact of CALI on survival in patients who underwent partial hepatectomy for colorectal liver metastases (CRLM).

\section{Methods}

Patients who received neoadjuvant chemotherapy and underwent partial hepatectomy for CRLM between 2008 and 2014 in a tertiary referral centre were included. Liver and tumour specimens were histologically examined for CALI (steatosis, steatohepatitis, sinusoidal dilatation, nodular regeneration) and tumour regression grade (TRG). TRG 1-2 was defined as complete tumour response.

\section{Results}

166 consecutive patients were included with a median follow-up of 47 and 60 months for recurrence-free and overall survival, respectively. Grade 2-3 sinusoidal dilatation was found in $44(26.5 \%)$ patients, grade $2-3$ steatosis in 48 (28.9\%), and steatohepatitis in one $(0.6 \%)$ patient. With hardly observed liver fibrosis, nodular regeneration was present in 25 out of 133 patients (18.8\%) with available collagen staining. Complete tumour response was observed in 33 (19.9\%) patients. Grade 2-3 sinusoidal dilatation, but not steatosis or nodular regeneration, was associated with diminished complete tumour response (odds ratio 3.99, 95\% Cl 1.17-13.65, p=0.027). CALI was not significantly related to survival. Complete tumour response was associated with prolonged recurrence-free (hazard ratio $0.47,95 \% \mathrm{Cl} 0.25-0.89, p=0.020$ ) and overall survival (hazard ratio $0.35,95 \% \mathrm{Cl} 0.18-0.68, p=0.002$ ).

\section{Conclusions}

CALI was not directly related to survival. CALI was, however, associated with diminished complete tumour response, and tumour response, in turn, was associated with survival. 


\section{Introduction}

Colorectal cancer is the third most common cancer worldwide, affecting over 1.3 million patients annually. ${ }^{1}$ Over 50 percent of these patients develop colorectal liver metastases (CRLM). ${ }^{2}$ Neoadjuvant chemotherapy is used to downstage the tumour and may facilitate future hepatic resection. ${ }^{3,4}$ Tumour downstaging relies on the tumour response to chemotherapy, as expressed in the tumour regression grade (TRG). CRLM patients with complete tumour response have been shown to have better survival than those with poor response. ${ }^{5}$

Systemic chemotherapy thus may have beneficial effects, but also frequently causes undesired liver parenchymal damage, collectively referred to as chemotherapyassociated liver injury (CALI). For instance, oxaliplatin-based treatment appears to be related to the development of sinusoidal obstruction syndrome ${ }^{6}$ and nodular regeneration, ${ }^{7}$ whereas irinotecan-based regimens are associated with increased occurrence of steatohepatitis. ${ }^{8-10}$ It has been shown by our group ${ }^{11}$ and subsequently by others $^{5}$ that CALI is associated with poor tumour response to neoadjuvant chemotherapy.

Despite the significant inverse relation between CALI and tumour response, few studies have been performed on this topic. ${ }^{5,11}$ Moreover, although one study claimed that chemotherapy-associated sinusoidal dilatation was associated with shortened survival, ${ }^{12}$ another study could not reproduce this. ${ }^{5}$ Therefore, it remains unclear whether CALI influences survival. The first aim of the present study was to validate the relation between CALI and tumour response in an independent large cohort. The second aim was to explore whether sinusoidal dilatation, nodular regeneration, steatosis, and steatohepatitis were associated with survival in CRLM patients after partial hepatectomy.

\section{Methods}

\section{Inclusion of patients}

Consecutive patients who had undergone partial hepatectomy for CRLM at Maastricht University Medical Centre between January 2008 and December 2013 were considered for this study. Inclusion criteria were: 1) patients treated with neoadjuvant chemotherapy; 2) availability of adequate histopathology assessment of non-tumour 
bearing liver tissue (i.e. presence of non-tumour-bearing liver at a distance of more than $2 \mathrm{~cm}$ from the tumour). Patients with cirrhosis were excluded from the study.

\section{Definition and data collection}

Comorbidity was defined as any disease affecting the patient apart from colorectal liver metastases (e.g. diabetes mellitus, and pulmonary, renal, cardiovascular, and other diseases). Overall morbidity was defined as any complication occurring within 30 days after surgery or during hospital stay and graded according to the classification of Dindo et al. ${ }^{13}$ Major morbidity was defined as Dindo-Clavien score Illa (requiring invasive intervention) or higher. The concept of a liver surgery-specific complication was in correspondence to the liver surgery-specific composite endpoint (CEP) developed in 2011, and included one or more of the following events: ascites, postoperative liver failure, bile leakage, intra-abdominal abscess, intra-abdominal haemorrhage, and operative mortality. ${ }^{14}$ Data regarding postoperative infectious complications were prospectively collected daily by an independent infection-control nurse (MR) based on the following definitions. Postoperative infectious complications were defined as a combination of surgical site infections, remote site infections, and systemic sepsis. Surgical site infections were defined according to the guideline of the Centres for Disease Control and Prevention (CDC)'s recommendations, including superficial incisional, deep incisional, and intra-abdominal organ/space surgical site infections. ${ }^{15}$ Remote site infections were defined as a condition in which fever and leukocytosis were present with bacteria in urine, sputum, or central venous catheter tips according to an experienced physician's judgment with consideration of microbiologic evidence when available. Radical resection was defined as resected tumour lesions with a surgical margin of more than one millimeter, and confirmed to have absent tumour cells on the margin from routine pathology reports.

\section{Pathology assessment of non-tumour-bearing liver and metastases}

Sinusoidal dilatation, nodular regeneration, steatosis, and tumour regression grade were semi-quantitatively assessed by two experienced liver pathologists (AW and CV). Diagnosis of nodular regeneration was made only when confirming liver fibrosis was absent or minor. Assessment of steatohepatitis was performed by another experienced liver pathologist (JV) independently. All pathologists were blinded to clinical information concerning the patients.

The tissue was fixed in formalin, embedded in paraffin, and stained with hematoxylin \& eosin and reticulin. Sinusoidal dilatation was graded according to Rubbia-Brandt et al. ${ }^{16}$ 
Nodular regeneration was graded according to the Wanless scoring system. ${ }^{17}$ Steatosis was graded according to Kleiner et al. ${ }^{18}$ A case presenting with at least grade 1 of each of the three features (steatosis, hepatocellular ballooning, and lobular inflammation) was classified as steatohepatitis. ${ }^{19}$ Tumour regression was graded as described by Mandard et $\mathrm{al}^{20}$ for the assessment of tumour regression after preoperative chemoradiotherapy for esophageal carcinoma, and modified for liver metastases. Grade 1 is characterized by the absence of histologically identifiable residual tumour and extensive fibrosis; grade 2 shows the presence of rare residual tumour cells scattered through the fibrosis; grade 3 represents a substantial amount of residual tumour cells but fibrosis dominated; grade 4 reflects residual tumour cells outgrowing fibrosis; and grade 5 indicates the absence of any tumour regression.

\section{Statistical Analyses}

All statistical analyses were performed using IBM SPSS Statistics for Windows (version 20.0). The relationship between CALI and tumour regression grade was studied applying uni- and multivariable binary logistic regression models. Furthermore, the association between CALI, tumour regression grade, infectious complications, and survival was analyzed using uni- and multivariable Cox regression models. Variables with $p$-value $\leq 0.10$ in univariable analysis were included in the multivariable analysis. Odds ratios (OR) or hazard ratios (HR) with $95 \%$ confidence intervals $(\mathrm{Cl})$ were calculated. A $p$-value $\leq 0.05$ was considered significant for all tests.

\section{Results}

A total of 166 consecutive patients were included (Table 5.1). Of these patients, 103 (62.0\%) were male and the median age was 65 (interquartile range 32-85) years. Preoperative comorbidity was found in 106 (63.9\%) patients. Nine (5.4\%) patients had undergone portal venous embolization before liver surgery. Ninety-nine (59.6\%) patients had more than one liver metastasis. Capecitabine in combination with oxaliplatin (CAPOX) was the most commonly used regimen, which was administered to 152 (91.6\%) patients. Bevacizumab was administered to 105 (63.3\%) patients. Postoperative surgical site infections were present in $41(30.7 \%)$ patients, and remote site infections were found in 26 (15.7\%) patients. 
Table $5.1 \quad$ Clinical characteristics.

\begin{tabular}{|c|c|}
\hline Factors & $n=166$ \\
\hline \multicolumn{2}{|l|}{ General characteristics } \\
\hline Sex (male) & $103(62.0 \%)$ \\
\hline Age (year) & $65(58-69)$ \\
\hline Body mass index $\left(\mathrm{kg} / \mathrm{m}^{2}\right)$ & $25.9(23.8-28.3)$ \\
\hline Comorbidity & $106(63.9 \%)$ \\
\hline Diabetes & $13(7.8 \%)$ \\
\hline Cardiovascular & $70(42.2 \%)$ \\
\hline Pulmonary & $18(10.8 \%)$ \\
\hline Renal & $6(3.6 \%)$ \\
\hline Rest & $39(23.5 \%)$ \\
\hline Portal venous embolization & $9(5.4 \%)$ \\
\hline \multicolumn{2}{|l|}{ Primary colorectal tumour } \\
\hline Rectal cancer & $72(43.4 \%)$ \\
\hline Tumour stage 3-4 $\left(n=165^{*}\right)$ & $144(87.3 \%)$ \\
\hline Lymph node with tumour cells $\left(n=162^{*}\right)$ & $113(69.8 \%)$ \\
\hline \multicolumn{2}{|l|}{ Liver metastases } \\
\hline Synchronous metastases (<12 months) & $130(78.3 \%)$ \\
\hline Number of metastases & $2(1-3)$ \\
\hline Diameter of largest metastases & $2.5(1.3-4.0)$ \\
\hline CEA >200 ng/ml at hepatectomy & $5(3.0 \%)$ \\
\hline \multicolumn{2}{|l|}{ Chemotherapy details } \\
\hline 5-fluorouracil & $6(3.6 \%)$ \\
\hline Oxaliplatin & $153(92.2 \%)$ \\
\hline Capecitabine & $152(91.6 \%)$ \\
\hline Irinotecan & $8(4.8 \%)$ \\
\hline Bevacizumab & $105(63.3 \%)$ \\
\hline \multicolumn{2}{|l|}{ Surgical details } \\
\hline Major hepatectomy ( $\geq 3$ Couinaud segments) & $78(47.0 \%)$ \\
\hline Pringle manoeuver & $44(26.5 \%)$ \\
\hline Laparoscopic surgery & $11(6.6 \%)$ \\
\hline Perioperative blood transfusion & $32(19.3 \%)$ \\
\hline Radical resection & $111(66.9 \%)$ \\
\hline \multicolumn{2}{|l|}{ Postoperative characteristics } \\
\hline Overall morbidity & $76(45.8 \%)$ \\
\hline Major morbidity (Dindo-Clavien III-V) & $29(17.5 \%)$ \\
\hline Liver surgery-specific complications & $32(19.3 \%)$ \\
\hline Infectious complications & $51(30.7 \%)$ \\
\hline Surgical site infections & $41(24.7 \%)$ \\
\hline Superficial wound & $22(13.3 \%)$ \\
\hline Deep wound & $6(3.6 \%)$ \\
\hline Intraabdominal organ/space & $27(16.3 \%)$ \\
\hline Remote site infections & $26(15.7 \%)$ \\
\hline Urinary tract & $12(7.2 \%)$ \\
\hline Respiratory tract & $15(9.0 \%)$ \\
\hline Central venous catheter-related & $6(3.6 \%)$ \\
\hline Sepsis & $13(7.8 \%)$ \\
\hline Ninety-day mortality & $5(3.0 \%)$ \\
\hline Length of hospital stay (day) & $8(6-10)$ \\
\hline
\end{tabular}

Numeric variables are presented as median with interquartile range; *Due to missing data, indicated number of patients was below 166; CEA, carcinoembryonic antigen. 
Table 5.2 summarizes the pathology details of chemotherapy-associated liver injury. Of the 90 (54.2\%) patients who presented with sinusoidal dilatation, mild lesions (grade 1 ) were found in $46(27.7 \%)$, and severe lesions (grade 2 or grade 3 ) in $44(26.5 \%)$ patients. Mild steatosis (grade 1) was found in 56 (33.7\%), and severe steatosis (grade 2 and grade 3 ) in 48 (28.9\%) patients. Collagen staining was available from 133 patients, of which nodular regeneration was found in 24 (18.5\%) patients. Steatohepatitis was present in one $(0.6 \%)$ patient, who had grade 2 steatosis, grade 2 hepatocellular ballooning, and grade 1 lobular inflammation. Complete tumour response (i.e. tumour regression grade $1-2)$ was observed in $33(19.8 \%)$ patients.

Table 5.2 Pathology characteristics.

\begin{tabular}{lc}
\hline Pathology details & $\mathrm{n}=166$ \\
\hline Sinusoidal dilatation & $76(45.8 \%)$ \\
Absent & $46(27.7 \%)$ \\
Grade 1 & $30(18.1 \%)$ \\
Grade 2 & $14(8.4 \%)$ \\
Grade 3 & \\
Nodular regeneration $\left(\mathrm{n}=133^{*}\right)$ & $108(65.1 \%)$ \\
Absent & $11(6.6 \%)$ \\
Grade 1 & $12(7.2 \%)$ \\
Grade 2 & $2(1.5 \%)$ \\
Grade 3 & \\
Steatosis & $62(37.3 \%)$ \\
Absent & $56(33.7 \%)$ \\
Grade 1 & $29(17.5 \%)$ \\
Grade 2 & $19(11.4 \%)$ \\
Grade 3 & $1(0.6 \%)$ \\
Steatohepatitis & \\
Tumour regression grade & $20(12.0 \%)$ \\
Grade 1 & $13(7.8 \%)$ \\
Grade 2 & $47(28.3 \%)$ \\
Grade 3 & $56(33.7 \%)$ \\
Grade 4 & $30(18.1 \%)$ \\
Grade 5 &
\end{tabular}

*Due to unavailable collagen staining, indicated number of patients was below 166.

The impact of CALI and other potentially important factors on tumour regression grade is depicted in Figure 5.1 (Detailed data are summarized in Supplemental Table S5.1). Given the fact that only one case of steatohepatitis was found, the relation between steatohepatitis and tumour regression grade could not be studied. Severe sinusoidal dilatation was associated with increased occurrence of diminished tumour response (OR 3.99, 95\% Cl 1.17-13.65, $p=0.027)$. An association between mild sinusoidal dilatation and diminished tumour response was statistically not significant (OR 2.38, 95\% Cl 0.83-6.85, $p=0.108$ ). Addition of bevacizumab to the chemotherapy treatment tended to be 
associated with increased complete tumour response (OR $0.35,95 \% \mathrm{Cl}$ 0.12-1.01, $p=0.052$ ). Severe steatosis ( $\mathrm{OR} 0.85,95 \% \mathrm{Cl} 0.33-2.21, p=0.741$ ) and severe nodular regeneration (OR 1.88, $95 \% \mathrm{Cl} 0.39-9.12, p=0.434$ ) were not related to tumour regression grade. In subgroup analyses, an inverse relation between the addition of bevacizumab and occurrence of severe sinusoidal dilatation (OR 0.35, 95\% Cl 0.17-0.76, $p=0.008$ ) was observed. Similarly, presence of severe steatosis was also inversely related to that of severe sinusoidal dilatation (OR $0.23,95 \% \mathrm{Cl} 0.08-0.66, p=0.006$ ) (Supplemental Table S5.2).

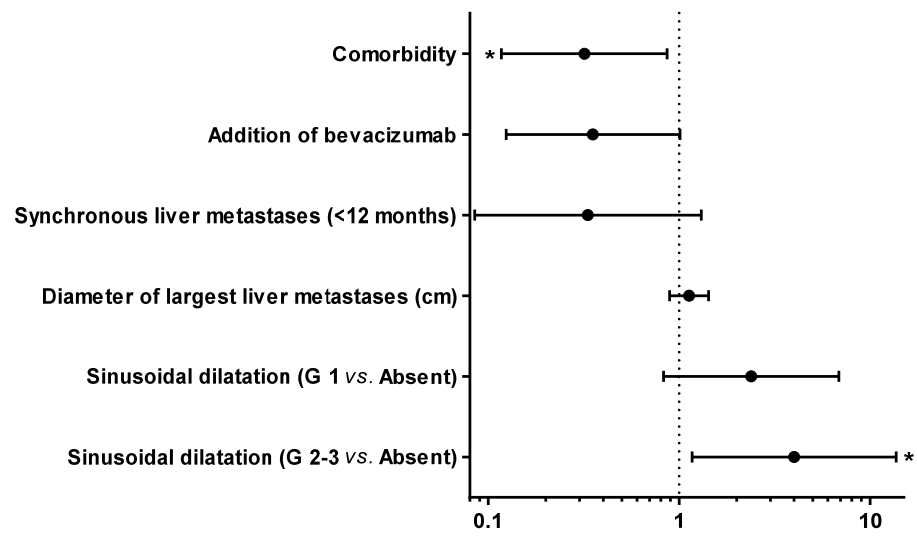

Figure 5.1 Factors related to tumour response.

Data are depicted as odds ratios (symbols) with 95\% confidence intervals (bars) based on results of multivariable analysis; asterisks indicate $p$-value $\leq 0.05$.

The relation between potential prognostic factors and recurrence-free survival is summarized in Figure 5.2 (Detailed data are summarized in Supplemental Table S5.3). Median follow-up for recurrence-free survival was 47 months. Both complete tumour response ( $\mathrm{HR} 0.47,95 \% \mathrm{Cl} 0.25-0.89, \mathrm{p}=0.020)$ and radical resection $(\mathrm{HR} 0.58,95 \% \mathrm{Cl}$ $0.38-0.88, p=0.011$ ) were associated with prolonged recurrence-free survival. Sinusoidal dilatation, steatosis, and nodular regeneration were not significantly associated with recurrence-free survival. The presence of remote site infections was the sole factor independently related to shortened recurrence-free survival ( $\mathrm{HR} 1.87,95 \% \mathrm{Cl} 1.06-3.30$, $p=0.030)$. 


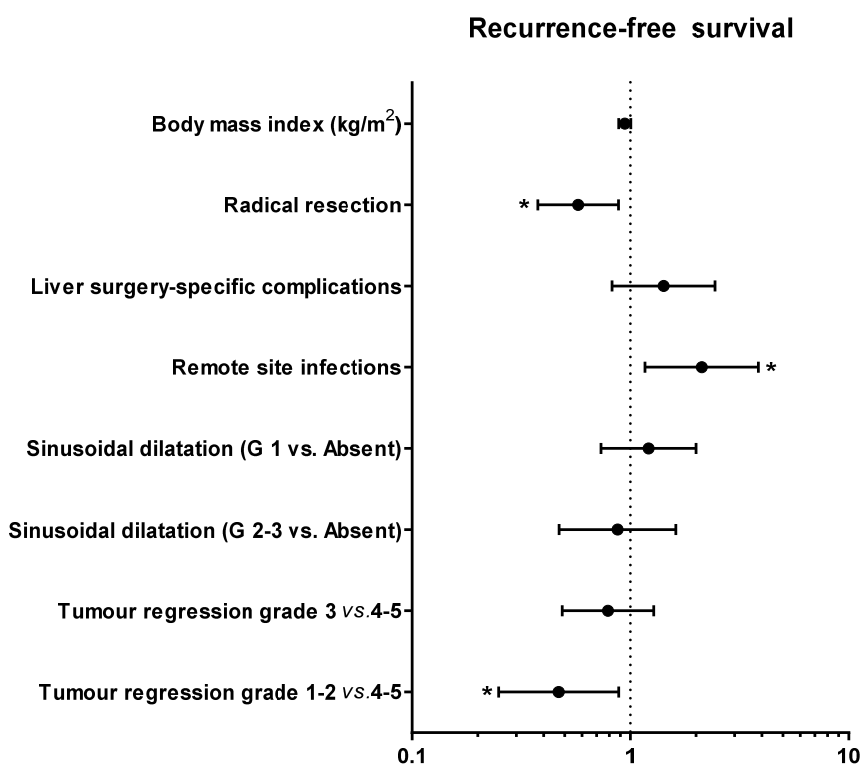

Figure 5.2 Prognostic factors related to recurrence-free survival.

Data are depicted as hazard ratios (symbols) with 95\% confidence intervals (bars) based on results of multivariable analysis, asterisks indicate $p$-value $\leq 0.05$.

Figure 5.3 summarizes the association between potential prognostic factors and overall survival (Detailed data are summarized in Supplemental Table S5.4). Median follow-up for overall survival was 60 months. Complete tumour response (i.e. tumour regression grade $1-2, \mathrm{HR} 0.35,95 \% \mathrm{Cl} 0.18-0.68, p=0.002)$ and radical resection $(\mathrm{HR} 0.52,95 \% \mathrm{Cl}$ $0.34-0.80, p=0.003$ ) were associated with prolonged overall survival. Again, sinusoidal dilatation, steatosis, and nodular regeneration were not significantly associated with overall survival. Synchronous (<12 months) liver metastases (HR 1.98, 95\% Cl 1.14-3.47, $p=0.016)$ and remote site infections ( $\mathrm{HR} 1.87,95 \% \mathrm{Cl} 1.06-3.30, p=0.030)$ were independently related to shortened overall survival. 


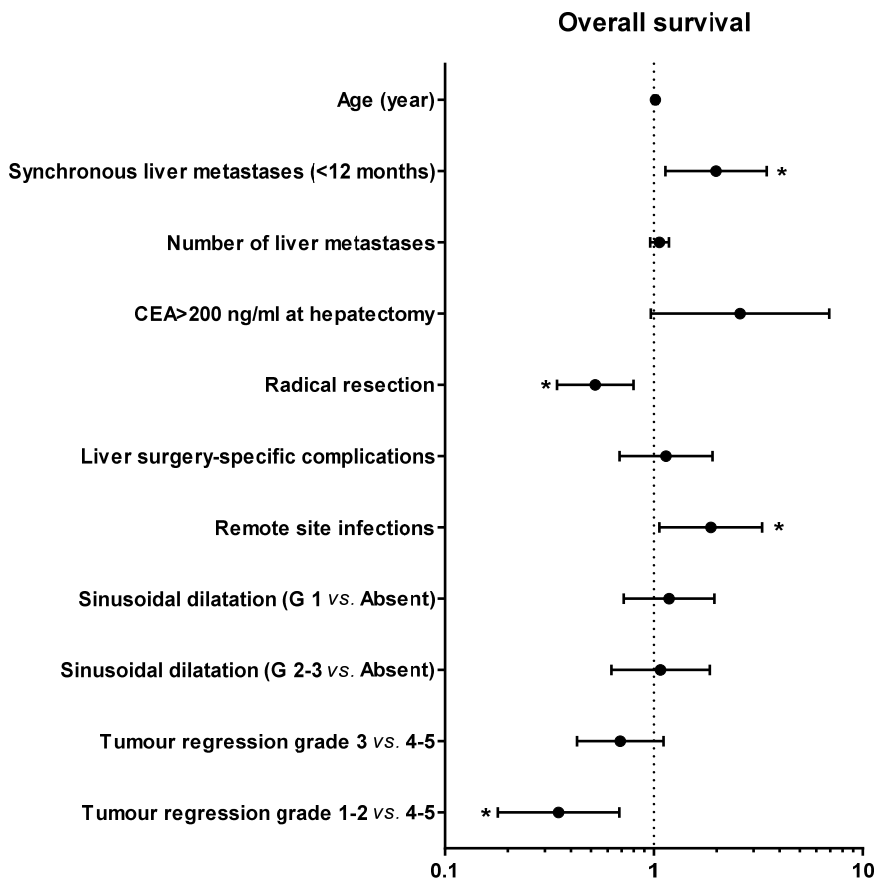

Figure 5.3 Prognostic factors related to overall survival.

Data are depicted as hazard ratios (symbols) with 95\% confidence intervals (bars) based on results of multivariable analysis; asterisks indicate $p$-value $\leq 0.05$.

\section{Discussion}

The data of the present study demonstrate that poor tumour response after neoadjuvant chemotherapy occurred more often in patients with sinusoidal dilatation, whereas neither steatosis nor nodular regeneration was associated with tumour response. Complete tumour response, in turn, was a strong independent predictor of prolonged recurrence-free survival and overall survival. However, none of the studied background liver abnormalities was independently related to survival. Addition of bevacizumab to chemotherapy was associated with decreased occurrence of sinusoidal dilatation, and tended to be related to increased complete tumour response. Moreover, remote site infections were related to reduced recurrence-free survival and overall survival, whereas radical resection was associated with an increase for these outcome parameters. 
Although sinusoidal dilatation was associated with diminished complete tumour response, which was associated with recurrence-free survival and overall survival, sinusoidal dilatation itself was not directly related to survival. As neoadjuvant chemotherapy provides increased complete tumour response, ${ }^{21}$ and this is related to prolonged survival, this observation might indicate that the benefits of neoadjuvant chemotherapy in terms of prolonging survival outweigh the harm of chemotherapyinduced undesired parenchymal liver injury. It is also suggested that chemotherapy could be more beneficial if clinicians could reduce the negative impact of CALI on tumour response. Since addition of bevacizumab to the chemotherapy treatment was associated with both a lower prevalence of sinusoidal dilatation and a tendency to increased tumour response, it seems beneficial to add bevacizumab to oxaliplatin-based chemotherapy.

In the present study, the previously shown ${ }^{5,11}$ inverse relation between chemotherapyassociated sinusoidal dilatation and tumour regression grade was confirmed. There may be several explanations for this finding. Loss of fenestrae in sinusoidal endothelial cells, which has been shown in an animal model of sinusoidal obstruction syndrome, ${ }^{22}$ might inhibit the transport of compounds ${ }^{23}$ such as oxaliplatin to the tumour, thereby limiting chemotherapy exposure. Oxaliplatin based chemotherapy is also known to lead to the detachment of sinusoidal endothelial cells from the space of Disse ${ }^{24}$, which may further weaken the hepatic microcirculation and limit transportation of drugs to the tumour. ${ }^{24,25}$ Moreover, a compromised hepatic microcirculation will lead to hypoperfusion and may induce a state of hepatic hypoxia. ${ }^{26}$ Tumour hypoxia will subsequently stimulate angiogenesis that may promote tumour cell invasion. ${ }^{27}$ Taken together, loss of fenestrae from sinusoidal endothelial cells, a weakened hepatic microcirculation, and hepatic hypoxia related to sinusoidal dilatation are all potential contributors to a diminished tumour response.

In line with a previous report ${ }^{28}$, the present study shows a negative relation between the presence of remote site infections and recurrence-free survival as well as overall survival. Although the mechanisms underlying this observation remain unclear, and a specific cause-effect relationship cannot be proven, we hypothesize that innate or adaptive immunity may play an important role. Patients with immune suppression suffer from increased occurrence of surgical infections. ${ }^{29}$ In keeping with this, enhancing the immune system by addition of thymosin ${ }^{30}$ inhibits tumour progression, ${ }^{31}$ and may enhance tumour response and prolong survival. ${ }^{32}$ In essence, the presence of infections may reflect a relatively weaker immune system. Weakened immunity may reduce 
tumour suppression and may eventually result in more frequent or earlier tumour recurrence and shortened survival.

Some limitations of this study should be discussed. Although analysis of the effect of steatohepatitis on tumour regression grade and survival would have been of interest, this was not possible since only one patient was diagnosed with steatohepatitis. This very low incidence of steatohepatitis could be due to the fact that only a small number of patients were treated with irinotecan, a known risk factor for the development of steatohepatitis. $^{8-10}$ Next, data on the interval between cessation of neoadjuvant chemotherapy and partial hepatectomy, as well as the number of administered cycles, were not available for the majority of included patients, since chemotherapy was frequently administered at local referral hospitals. Therefore, the influence of these factors on tumour regression grade and survival could not be evaluated. Despite this limitation, it must be highlighted that the majority of the studied population received the same regimen, which was capecitabine in combination with oxaliplatin. Therefore, the effect of treatment regimen related factors on tumour regression grade and survival was presumably minimal.

\section{Conclusion}

This study demonstrated that chemotherapy-associated sinusoidal dilatation is related to diminished complete tumour response after neoadjuvant chemotherapy. Complete tumour response was shown to be the strongest predictor of prolonged recurrence-free survival and overall survival. None of the background parenchymal chemotherapyinduced liver injuries was associated with survival. Addition of bevacizumab was associated with decreased sinusoidal dilatation, and tended to be related to increased complete tumour response. Given that oxaliplatin-based treatment was associated with increased sinusoidal dilatation, ${ }^{6}$ and that sinusoidal dilatation was related to diminished tumour response, it seems advisable to co-administer bevacizumab with oxaliplatinbased regimens. Furthermore, since infections were related to reduced survival, application of meticulous aseptic surgery techniques and infection prevention protocols may be of critical importance for improving long-term outcome. 


\section{References}

1. Ferlay J, Soerjomataram I, Dikshit R, et al. Cancer incidence and mortality worldwide: sources, methods and major patterns in GLOBOCAN 2012. Int J Cancer 2015; 136(5):E359-86.

2. Kanas GP, Taylor A, Primrose JN, et al. Survival after liver resection in metastatic colorectal cancer: review and meta-analysis of prognostic factors. Clin Epidemiol 2012; 4:283-301.

3. Lam VW, Spiro C, Laurence JM, et al. A systematic review of clinical response and survival outcomes of downsizing systemic chemotherapy and rescue liver surgery in patients with initially unresectable colorectal liver metastases. Ann Surg Oncol 2012; 19(4):1292-301.

4. Adam R, Delvart V, Pascal G, et al. Rescue surgery for unresectable colorectal liver metastases downstaged by chemotherapy: a model to predict long-term survival. Ann Surg 2004; 240(4):644-57; discussion 657-8.

5. Vigano L, Capussotti L, De Rosa G, et al. Liver resection for colorectal metastases after chemotherapy: impact of chemotherapy-related liver injuries, pathological tumor response, and micrometastases on long-term survival. Ann Surg 2013; 258(5):731-40; discussion 741-2.

6. Rubbia-Brandt L, Lauwers GY, Wang H, et al. Sinusoidal obstruction syndrome and nodular regenerative hyperplasia are frequent oxaliplatin-associated liver lesions and partially prevented by bevacizumab in patients with hepatic colorectal metastasis. Histopathology 2010; 56(4):430-9.

7. Vigano L, Rubbia-Brandt L, De Rosa G, et al. Nodular Regenerative Hyperplasia in Patients Undergoing Liver Resection for Colorectal Metastases After Chemotherapy: Risk Factors, Preoperative Assessment and Clinical Impact. Ann Surg Oncol 2015; 22(13):4149-57.

8. Morris-Stiff G, Tan YM, Vauthey JN. Hepatic complications following preoperative chemotherapy with oxaliplatin or irinotecan for hepatic colorectal metastases. Eur J Surg Oncol 2008; 34(6):609-14.

9. Khan AZ, Morris-Stiff G, Makuuchi M. Patterns of chemotherapy-induced hepatic injury and their implications for patients undergoing liver resection for colorectal liver metastases. J Hepatobiliary Pancreat Surg 2009; 16(2):137-44.

10. Robinson $\mathrm{SM}$, Wilson $\mathrm{CH}$, Burt $\mathrm{AD}$, et al. Chemotherapy-associated liver injury in patients with colorectal liver metastases: a systematic review and meta-analysis. Ann Surg Oncol 2012; 19(13):4287-99.

11. Vreuls CP, Van Den Broek MA, Winstanley A, et al. Hepatic sinusoidal obstruction syndrome (SOS) reduces the effect of oxaliplatin in colorectal liver metastases. Histopathology 2012; 61(2):314-8.

12. TamandI D, Klinger M, Eipeldauer S, et al. Sinusoidal obstruction syndrome impairs long-term outcome of colorectal liver metastases treated with resection after neoadjuvant chemotherapy. Ann Surg Oncol 2011; 18(2):421-30.

13. Dindo D, Demartines N, Clavien PA. Classification of surgical complications: a new proposal with evaluation in a cohort of 6336 patients and results of a survey. Ann Surg 2004; 240(2):205-13.

14. van den Broek MA, van Dam RM, van Breukelen GJ, et al. Development of a composite endpoint for randomized controlled trials in liver surgery. Br J Surg 2011; 98(8):1138-45.

15. Mangram AJ, Horan TC, Pearson ML, et al. Guideline for Prevention of Surgical Site Infection, 1999. Centers for Disease Control and Prevention (CDC) Hospital Infection Control Practices Advisory Committee. Am J Infect Control 1999; 27(2):97-132; quiz 133-4; discussion 96.

16. Rubbia-Brandt L, Audard V, Sartoretti $P$, et al. Severe hepatic sinusoidal obstruction associated with oxaliplatin-based chemotherapy in patients with metastatic colorectal cancer. Ann Oncol 2004; 15(3):460-6.

17. Wanless IR. Micronodular transformation (nodular regenerative hyperplasia) of the liver: a report of 64 cases among 2,500 autopsies and a new classification of benign hepatocellular nodules. Hepatology 1990; 11(5):787-97.

18. Kleiner DE, Brunt EM, Van Natta M, et al. Design and validation of a histological scoring system for nonalcoholic fatty liver disease. Hepatology 2005; 41(6):1313-21.

19. Bedossa P, Consortium FP. Utility and appropriateness of the fatty liver inhibition of progression (FLIP) algorithm and steatosis, activity, and fibrosis (SAF) score in the evaluation of biopsies of nonalcoholic fatty liver disease. Hepatology 2014; 60(2):565-75.

20. Mandard AM, Dalibard F, Mandard JC, et al. Pathologic assessment of tumor regression after preoperative chemoradiotherapy of esophageal carcinoma. Clinicopathologic correlations. Cancer 1994; 73(11):2680-6. 
21. Folprecht G, Gruenberger T, Bechstein WO, et al. Tumour response and secondary resectability of colorectal liver metastases following neoadjuvant chemotherapy with cetuximab: the CELIM randomised phase 2 trial. Lancet Oncol 2010; 11(1):38-47.

22. Deleve LD, McCuskey RS, Wang X, et al. Characterization of a reproducible rat model of hepatic venoocclusive disease. Hepatology 1999; 29(6):1779-91.

23. Perri RE, Shah V. Hepatic Sinusoidal Endothelial Cells. In: Dufour J-F, Clavien P-A, Trautwein C, Graf R, eds. Signaling Pathways in Liver Diseases. Berlin, Heidelberg: Springer Berlin Heidelberg; 2005:pp. 53-62.

24. Vreuls CP, Driessen A, Olde Damink SW, et al. Sinusoidal obstruction syndrome (SOS): A light and electron microscopy study in human liver. Micron 2016; 84:17-22.

25. DeLeve LD, Shulman HM, McDonald GB. Toxic injury to hepatic sinusoids: sinusoidal obstruction syndrome (veno-occlusive disease). Semin Liver Dis 2002; 22(1):27-42.

26. Rubbia-Brandt L, Tauzin S, Brezault C, et al. Gene expression profiling provides insights into pathways of oxaliplatin-related sinusoidal obstruction syndrome in humans. Mol Cancer Ther 2011; 10(4):687-96.

27. Allen $\mathrm{M}$, Louise Jones J. Jekyll and Hyde: the role of the microenvironment on the progression of cancer. J Pathol 2011; 223(2):162-76.

28. Artinyan A, Orcutt ST, Anaya DA, et al. Infectious postoperative complications decrease long-term survival in patients undergoing curative surgery for colorectal cancer: a study of 12,075 patients. Ann Surg 2015; 261(3):497-505.

29. Akhter MS, Verma R, Madhukar KP, et al. Incidence of surgical site infection in postoperative patients at a tertiary care centre in India. J Wound Care 2016; 25(4):210-2, 214-7.

30. Silecchia G, Guarino E, Sinibaldi-Vallebona P, et al. Efficacy of repeated cycles of chemo-immunotherapy with thymosin alpha1 and interleukin-2 after intraperitoneal 5-fluorouracil delivery. Cancer Immunol Immunother 1999; 48(4):172-8.

31. Shrivastava P, Singh SM, Singh N. Effect of thymosin alpha 1 on the antitumor activity of tumorassociated macrophage-derived dendritic cells. J Biomed Sci 2004; 11(5):623-30.

32. Lipson SD, Chretien PB, Makuch R, et al. Thymosin immunotherapy in patients with small cell carcinoma of the lung: correlation of in vitro studies with clinical course. Cancer 1979; 43(3):863-70. 


\section{Supplemental tables}

Table S5.1 Factors related to tumour response.

\begin{tabular}{|c|c|c|c|c|}
\hline \multirow[b]{3}{*}{ Factors } & \multicolumn{4}{|c|}{ Tumour regression grade $3-5$} \\
\hline & \multicolumn{2}{|c|}{ univariable } & \multicolumn{2}{|c|}{ multivariable } \\
\hline & OR $(95 \% \mathrm{Cl})$ & $\mathrm{p}$-value & OR $(95 \% \mathrm{Cl})$ & $\mathrm{p}$-value \\
\hline \multicolumn{5}{|l|}{ General characteristics } \\
\hline Sex (male) & $1.43(0.65-3.14)$ & 0.371 & - & - \\
\hline Age (year) & $1.00(0.96-1.04)$ & 0.983 & - & - \\
\hline Body mass index (kg/m2) & $1.00(0.90-1.12)$ & 0.941 & - & - \\
\hline Comorbidity & $0.47(0.19-1.12)$ & 0.087 & $0.32(0.12-0.86)$ & 0.024 \\
\hline Portal venous embolization & n.a.* & n.a.* & - & - \\
\hline \multicolumn{5}{|l|}{ Primary colorectal tumour } \\
\hline Rectal cancer & $0.60(0.27-1.31)$ & 0.197 & - & - \\
\hline Tumour stage 3-4 & $2.16(0.78-6.02)$ & 0.139 & - & - \\
\hline Lymph node with tumour cells & $0.64(0.26-1.56)$ & 0.323 & - & - \\
\hline \multicolumn{5}{|l|}{ Liver metastases } \\
\hline Synchronous (<12 months) & $0.25(0.07-0.88)$ & 0.032 & $0.33(0.09-1.30)$ & 0.114 \\
\hline Number of metastases & $1.02(0.84-1.24)$ & 0.850 & - & - \\
\hline Diameter of largest metastases (cm) & $1.29(1.02-1.64)$ & 0.037 & $1.13(0.89-1.43)$ & 0.326 \\
\hline CEA > $200 \mathrm{ng} / \mathrm{ml}$ at hepatectomy & n.a. $\neq$ & n.a. $\neq$ & - & - \\
\hline \multicolumn{5}{|l|}{ Chemotherapy } \\
\hline Addition of bevacizumab & $0.30(0.12-0.80)$ & 0.015 & $0.35(0.12-1.01)$ & 0.052 \\
\hline \multicolumn{5}{|l|}{$\begin{array}{l}\text { Chemotherapy-associated liver injury } \\
\text { Sinusoidal dilatation }\end{array}$} \\
\hline Absent & 1 & & 1 & \\
\hline Grade 1 & $2.46(0.92-6.58)$ & 0.073 & $2.38(0.83-6.85)$ & 0.108 \\
\hline Grade 2-3 & $4.36(1.44-13.21)$ & 0.009 & $3.99(1.17-13.65)$ & 0.027 \\
\hline \multicolumn{5}{|l|}{ Steatosis } \\
\hline Absent & 1 & & & \\
\hline Grade 1 & $0.97(0.37-2.50)$ & 0.942 & - & - \\
\hline Grade 2-3 & $0.85(0.33-2.21)$ & 0.741 & - & - \\
\hline Nodular regeneration & $2.30(0.63-8.43)$ & 0.208 & - & - \\
\hline Absent & 1 & & & \\
\hline Grade 1 & $3.14(0.38-26.17)$ & 0.289 & - & - \\
\hline Grade 2-3 & $1.88(0.39-9.12)$ & 0.434 & - & - \\
\hline
\end{tabular}

*The proportion of patients with complete tumour response is $0 \%$ for patients with portal venous embolization and $100 \%$ for those without (Fisher's exact test: $p=0.258$; $\neq$ the proportion of patients with complete tumour response is $0 \%$ for patients with CEA $>200 \mathrm{ng} / \mathrm{ml}$ and $100 \%$ for those with CEA $\leq 200 \mathrm{ng} / \mathrm{ml}$ (Fisher's exact test: $\mathrm{p}=0.650)$; CEA, carcinoembryonic antigen. 
Table S5.2 Factors related to sinusoidal dilatation.

\begin{tabular}{|c|c|c|c|c|}
\hline \multirow[b]{3}{*}{ Factors } & \multicolumn{4}{|c|}{ Sinusoidal dilatation grade $2-3$} \\
\hline & \multicolumn{2}{|l|}{ univariable } & \multicolumn{2}{|c|}{ multivariable } \\
\hline & OR $(95 \% \mathrm{Cl})$ & $\mathrm{p}$-value & OR $(95 \% \mathrm{Cl})$ & $\mathrm{p}$-value \\
\hline \multicolumn{5}{|l|}{ General characteristics } \\
\hline Sex (male) & $1.16(0.56-2.41)$ & 0.693 & - & - \\
\hline Age (year)* & $1.03(0.99-1.08)$ & 0.101 & $1.04(1.00-1.08)$ & 0.070 \\
\hline Body mass index (kg/m2) & $1.01(0.92-1.12)$ & 0.824 & - & - \\
\hline Comorbidity & $1.46(0.68-3.12)$ & 0.331 & - & - \\
\hline Portal venous embolization & $2.20(0.49-9.96)$ & 0.307 & - & - \\
\hline \multicolumn{5}{|l|}{ Chemotherapy } \\
\hline Oxaliplatin & $1.33(0.27-6.61)$ & 0.732 & - & - \\
\hline Capecitabine & $0.84(0.24-2.95)$ & 0.790 & - & - \\
\hline Irinotecan & $2.49(0.57-10.82)$ & 0.223 & - & - \\
\hline 5-fluorouracil & $0.90(0.16-5.21)$ & 0.906 & - & - \\
\hline Addition of bevacizumab & $0.34(0.16-0.71)$ & 0.004 & $0.35(0.17-0.76)$ & 0.008 \\
\hline \multicolumn{5}{|l|}{$\begin{array}{l}\text { Chemotherapy-associated liver injury } \\
\text { Steatosis }\end{array}$} \\
\hline Absent & 1 & & 1 & \\
\hline Grade 1 & $0.55(0.24-1.26)$ & 0.156 & $0.50(0.19-1.09)$ & 0.077 \\
\hline Grade 2-3 & $0.24(0.09-0.65)$ & 0.005 & $0.23(0.08-0.66)$ & 0.006 \\
\hline \multicolumn{5}{|c|}{ Nodular regeneration $(n=133) \neq \emptyset$} \\
\hline Absent & 1 & & - & \\
\hline Grade 1 & $3.32(0.87-12.62)$ & 0.079 & - & - \\
\hline Grade $2-3$ & $11.05(2.90-42.11)$ & 0.0004 & - & - \\
\hline
\end{tabular}

*Considering the p-value in univariable analysis reached borderline of 0.10 , age was included in the multivariable analysis; ¥Due to missing data, indicated number of patients was below 166; IBecause of the suspected collinearity between sinusoidal dilatation and nodular regeneration, nodular regeneration was not included into multivariable analysis. 
Table S5.3 Prognostic factors related to recurrence-free survival.

\begin{tabular}{|c|c|c|c|c|}
\hline \multirow[b]{3}{*}{ Factors } & \multicolumn{4}{|c|}{ Recurrence-free survival } \\
\hline & \multicolumn{2}{|c|}{ univariable } & \multicolumn{2}{|c|}{ multivariable } \\
\hline & $\mathrm{HR}(95 \% \mathrm{Cl})$ & p-value & $\mathrm{HR}(95 \% \mathrm{Cl})$ & p-value \\
\hline \multicolumn{5}{|l|}{ General characteristics } \\
\hline Sex (male) & $1.07(0.70-1.63)$ & 0.768 & - & - \\
\hline Age (year) & $1.00(0.98-1.02)$ & 0.958 & - & - \\
\hline Body mass index $\left(\mathrm{kg} / \mathrm{m}^{2}\right)$ & $0.95(0.89-1.01)$ & 0.079 & $0.94(0.89-1.00)$ & 0.067 \\
\hline Comorbidity & $1.30(0.86-1.98)$ & 0.219 & - & - \\
\hline Portal venous embolization & $0.86(0.35-2.12)$ & 0.734 & - & - \\
\hline \multicolumn{5}{|l|}{ Primary colorectal tumour } \\
\hline Rectal cancer & $1.15(0.77-1.72)$ & 0.496 & - & - \\
\hline Tumour stage 3-4 & $1.65(0.88-3.11)$ & 0.122 & - & - \\
\hline Lymph node with tumour cells & $1.37(0.88-2.14)$ & 0.169 & - & - \\
\hline \multicolumn{5}{|l|}{ Liver metastases } \\
\hline Synchronous (<12 months) & $1.42(0.86-2.33)$ & 0.168 & - & - \\
\hline Number of metastases & $1.06(0.95-1.18)$ & 0.297 & - & - \\
\hline Diameter of largest metastases (cm) & $1.02(0.93-1.12)$ & 0.695 & - & - \\
\hline CEA >200 ng/ml at hepatectomy & $1.92(0.78-4.74)$ & 0.158 & - & - \\
\hline \multicolumn{5}{|l|}{ Chemotherapy } \\
\hline Addition of bevacizumab & $0.87(0.57-1.32)$ & 0.508 & - & - \\
\hline \multicolumn{5}{|l|}{ Surgical details } \\
\hline Major hepatectomy ( $\geq 3$ Couinaud segments) & $0.79(0.53-1.18)$ & 0.245 & - & - \\
\hline Pringle manoeuver & $1.08(0.69-1.70)$ & 0.737 & - & - \\
\hline Laparoscopic surgery & $1.30(0.56-2.99)$ & 0.544 & - & - \\
\hline Perioperative blood transfusion & $1.14(0.67-1.94)$ & 0.639 & - & - \\
\hline Radical resection & $0.52(0.34-0.78)$ & 0.002 & $0.58(0.38-0.88)$ & 0.011 \\
\hline \multicolumn{5}{|l|}{ Postoperative morbidity } \\
\hline Overall morbidity & $1.40(0.93-2.10)$ & 0.105 & - & - \\
\hline Major morbidity (Dindo-Clavien III-V) & $1.55(0.88-2.70)$ & 0.127 & - & - \\
\hline Liver surgery-specific complications & $1.72(1.04-2.83)$ & 0.034 & $1.42(0.82-2.44)$ & 0.207 \\
\hline Surgical site infections & $1.30(0.82-2.07)$ & 0.266 & - & - \\
\hline Remote site infections & $2.29(1.38-3.81)$ & 0.001 & $2.12(1.17-3.86)$ & 0.014 \\
\hline \multicolumn{5}{|l|}{ Chemotherapy-associated liver injury } \\
\hline \multicolumn{5}{|l|}{ Sinusoidal dilatation } \\
\hline Absent & 1 & & 1 & \\
\hline Grade 1 & $1.59(0.98-2.58)$ & 0.060 & $1.21(0.73-2.00)$ & 0.454 \\
\hline Grade $2-3$ & $1.41(0.83-2.42)$ & 0.208 & $0.87(0.47-1.62)$ & 0.668 \\
\hline \multicolumn{5}{|l|}{ Steatosis } \\
\hline Absent & 1 & & & \\
\hline Grade 1 & $1.15(0.71-1.86)$ & 0.566 & - & - \\
\hline Grade 2-3 & $0.96(0.59-1.59)$ & 0.886 & - & - \\
\hline \multicolumn{5}{|l|}{ Nodular regeneration } \\
\hline Absent & 1 & & & \\
\hline Grade 1 & $0.68(0.29-1.58)$ & 0.366 & - & - \\
\hline Grade 2-3 & $1.02(0.51-2.05)$ & 0.946 & - & - \\
\hline \multicolumn{5}{|l|}{ Tumour response } \\
\hline \multicolumn{5}{|l|}{ Tumour regression grade } \\
\hline Grade $4-5$ & 1 & & & \\
\hline Grade 3 & $0.97(0.61-1.55)$ & 0.907 & $0.79(0.49-1.28)$ & 0.337 \\
\hline Grade 1-2 & $0.51(0.28-0.92)$ & 0.026 & $0.47(0.25-0.89)$ & 0.020 \\
\hline
\end{tabular}

CEA, carcinoembryonic antigen. 
Table S5.4 Prognostic factors related to overall survival.

\begin{tabular}{|c|c|c|c|c|}
\hline \multirow[b]{3}{*}{ Factors } & \multicolumn{4}{|c|}{ Overall survival } \\
\hline & \multicolumn{2}{|c|}{ univariable } & \multicolumn{2}{|c|}{ multivariable } \\
\hline & $\mathrm{HR}(95 \% \mathrm{Cl})$ & p-value & $\mathrm{HR}(95 \% \mathrm{CI})$ & $\mathrm{p}$-value \\
\hline \multicolumn{5}{|l|}{ General characteristics } \\
\hline Sex (male) & $1.04(0.69-1.55)$ & 0.867 & - & - \\
\hline Age (year) & $1.02(1.00-1.04)$ & 0.064 & $1.02(0.99-1.04)$ & 0.180 \\
\hline Body mass index $\left(\mathrm{kg} / \mathrm{m}^{2}\right)$ & $0.96(0.91-1.02)$ & 0.165 & - & - \\
\hline Comorbidity & $1.10(0.73-1.64)$ & 0.655 & - & - \\
\hline Portal venous embolization & $0.98(0.40-2.43)$ & 0.972 & - & - \\
\hline \multicolumn{5}{|l|}{ Primary colorectal tumour } \\
\hline Rectal cancer & $0.98(0.66-1.45)$ & 0.919 & - & - \\
\hline Tumour stage 3-4 & $1.52(0.79-2.92)$ & 0.214 & - & - \\
\hline Lymph node with tumour cells & $1.21(0.78-1.87)$ & 0.390 & - & - \\
\hline \multicolumn{5}{|l|}{ Liver metastases } \\
\hline Synchronous (<12 months) & $1.67(1.00-2.79)$ & 0.051 & $1.98(1.14-3.47)$ & 0.016 \\
\hline Number of metastases & $1.10(1.00-1.21)$ & 0.050 & $1.06(0.96-1.18)$ & 0.245 \\
\hline Diameter of largest metastases (cm) & $1.01(0.92-1.11)$ & 0.906 & - & - \\
\hline CEA >200 ng/ml at hepatectomy & $2.67(1.08-6.58)$ & 0.064 & $2.59(0.97-6.92)$ & 0.058 \\
\hline \multicolumn{5}{|l|}{ Chemotherapy } \\
\hline Addition of bevacizumab & $0.94(0.63-1.40)$ & 0.747 & - & - \\
\hline \multicolumn{5}{|l|}{ Surgical details } \\
\hline Major hepatectomy ( $\geq 3$ Couinaud segments) & $0.80(0.54-1.19)$ & 0.268 & - & - \\
\hline Pringle manoeuver & $1.03(0.66-1.61)$ & 0.885 & - & - \\
\hline Laparoscopic surgery & $0.76(0.31-1.86)$ & 0.544 & - & - \\
\hline Perioperative blood transfusion & $1.06(0.65-1.73)$ & 0.815 & - & - \\
\hline Radical resection & $0.44(0.30-0.66)$ & $<0.001$ & $0.52(0.34-0.80)$ & 0.003 \\
\hline \multicolumn{5}{|l|}{ Postoperative morbidity } \\
\hline Overall morbidity & $1.33(0.90-1.96)$ & 0.156 & - & - \\
\hline Major morbidity (Dindo-Clavien III-V) & $1.16(0.70-1.93)$ & 0.568 & - & - \\
\hline Liver surgery-specific complications & $1.49(0.94-2.38)$ & 0.092 & $1.14(0.69-1.91)$ & 0.605 \\
\hline Surgical site infections & $1.34(0.86-2.10)$ & 0.195 & - & - \\
\hline Remote site infections & $1.79(1.08-2.97)$ & 0.023 & $1.87(1.06-3.30)$ & 0.030 \\
\hline \multicolumn{5}{|l|}{ Chemotherapy-associated liver injury } \\
\hline \multicolumn{5}{|l|}{ Sinusoidal dilatation } \\
\hline Absent & 1 & & & \\
\hline Grade 1 & $1.54(0.95-2.47)$ & 0.078 & $1.18(0.72-1.95)$ & 0.509 \\
\hline Grade $2-3$ & $1.59(0.97-2.60)$ & 0.067 & $1.08(0.63-1.85)$ & 0.789 \\
\hline \multicolumn{5}{|l|}{ Steatosis } \\
\hline Absent & 1 & & & \\
\hline Grade 1 & $1.20(0.75-1.93)$ & 0.447 & - & - \\
\hline Grade 2-3 & $0.86(0.52-1.42)$ & 0.555 & - & - \\
\hline Nodular regeneration & & & - & - \\
\hline Absent & 1 & & & \\
\hline Grade 1 & $1.19(0.56-2.51)$ & 0.651 & - & - \\
\hline Grade 2-3 & $0.79(0.38-1.64)$ & 0.518 & - & - \\
\hline \multicolumn{5}{|l|}{ Tumour response } \\
\hline \multicolumn{5}{|l|}{ Tumour regression grade } \\
\hline Grade $4-5$ & 1 & & 1 & \\
\hline Grade 3 & $0.73(0.46-1.14)$ & 0.167 & $0.69(0.43-1.11)$ & 0.129 \\
\hline Grade 1-2 & $0.34(0.18-0.64)$ & 0.001 & $0.35(0.18-0.68)$ & 0.002 \\
\hline
\end{tabular}

CEA, carcinoembryonic antigen. 


\section{Part II}

Mechanisms underlying chemotherapy -associated liver injury 



\section{Chapter}

\section{miR-21 and miR-150 downregulation is associated with oxaliplatin-induced sinusoidal dilatation and impaired survival}

Junfang Zhao, Sander S. Rensen, Celien P.H. Vreuls, Maartje van den Broek, Ann Driessen, Marcel van Herwijnen, Marlon J.A. Jetten, Danyel G.J. Jennen, Cornelis H.C. Dejong, Steven W.M. Olde Damink 


\section{Abstract}

\section{Background}

Oxaliplatin-induced sinusoidal dilatation (SD) is associated with increased morbidity and impaired survival. Hence, identification of the molecular mechanisms underpinning the development of SD is pivotal.

\section{Methods}

This case-matched cohort study of patients who underwent partial hepatectomy for colorectal liver metastases between 2008 and 2009 included 28 patients with preoperative oxaliplatin treatment and SD (grade 2-3 versus absent); ten patients with grade 2-3 SD were matched with ten without SD by number of oxaliplatin cycles, cumulative oxaliplatin dose, sex, age, body mass index, and liver transaminases. Nontumour liver microRNA expression was analyzed using Agilent arrays for the 20 patients that could be matched and validated by quantitative PCR for all 28 patients included. MicroRNA expression was subsequently related to overall survival and recurrence free survival.

\section{Results}

Grade 2-3 SD was not associated with upregulation of any microRNA. However, miR-21 and miR-150 were significantly downregulated in the grade 2-3 SD group (array: 1.34fold, $p=0.028$; 1.46 -fold, $p=0.035$, respectively; $q P C R$ : 1.32-fold, $p=0.001 ; 1.34$-fold, $p=0.014$, respectively). Low hepatic expression of both miR-21 and miR-150 was associated with impaired overall survival $(n=28, p=0.032$ and $p=0.010$, respectively). Likewise, both overall recurrence-free survival and liver recurrence-free survival were reduced in subjects with low hepatic miR-21 ( $p=0.010$ and $p=0.046$, respectively) and miR-150 expression ( $p=0.025$ and $p=0.031$, respectively).

\section{Conclusions}

The strong association between reduced hepatic miR-21/miR-150 levels and SD, tumour recurrence, and survival indicates that miR-21 and miR-150 regulated genes may be involved in the pathogenesis of SD and may be used as prognostic markers. 


\section{Introduction}

Colorectal cancer is the third most common cancer in men and second in women worldwide, affecting over 1.3 million patients annually. ${ }^{1}$ The liver is the dominant metastatic site, and approximately $50 \%$ of patients develop colorectal liver metastases (CRLM). ${ }^{2}$ Although liver resection is the only potentially curative treatment, only $10-30 \%$ of patients with liver metastases are candidates for hepatic surgery. ${ }^{2}$ Inoperability is mainly caused by patient comorbidity in combination with number and size of hepatic tumour. For patients who have unresectable metastases, chemotherapy is currently the most commonly used treatment to prolong survival.

For decades, 5-fluorouracil was the sole active chemotherapeutic agent for CRLM. This has changed markedly since 2000: with the approval of irinotecan, oxaliplatin, and humanized monoclonal antibodies, many more initially unresectable CRLM became resectable. ${ }^{3}$ Although response rates are convincing administration of these agents is associated with significant liver injury. More specifically, the administration of irinotecan appears to be related to the development of nonalcoholic steatohepatitis, whilst oxaliplatin may lead to the development of sinusoidal obstruction syndrome. ${ }^{4}$

Chemotherapy-associated sinusoidal obstruction syndrome in patients with CRLM is characterized by sinusoidal dilatation (SD), centrilobular vein injury, centrilobular fibrosis, and peliosis. ${ }^{5}$ SD is the hallmark feature and routinely used to evaluate the severity of sinusoidal obstruction syndrome. ${ }^{5}$ We and others previously showed that the development of SD diminishes the tumour response to chemotherapy. ${ }^{6,7}$ SD has also been shown to increase postoperative morbidity ${ }^{8}$ and compromise postoperative recurrence-free survival and overall survival in patients undergoing liver surgery for CRLM. ${ }^{9}$

In view of these clinical observations and the lack of treatment options for SD, it is of key importance to identify the molecular mechanisms underlying its development. Recent mRNA microarray-based approaches showed potential involvement of angiogenesis, coagulation, the acute phase response, hepatic fibrosis, oxidative stress, and hypoxia in SD. ${ }^{10}$ However, expression profiles of microRNAs, which function as regulators of gene expression through translational repression or mRNA cleavage, have not yet been investigated in the context of SD. Nevertheless, microRNAs are known to play important roles in tumour angiogenesis and progression ${ }^{11}$ and have been linked to survival in patients with colorectal cancer. ${ }^{12}$ Furthermore, the concentration of several microRNAs has been shown to be enriched in the serum of patients with drug-induced liver injury. ${ }^{13}$ 
Moreover, specific circulating microRNAs have been shown to represent reliable biomarkers for predicting prognosis and the tumour response to chemotherapeutic drugs. ${ }^{14,15}$ In addition, targeting a specific microRNA involved in the pathogenesis of liver disease is a feasible and effective treatment option. ${ }^{16}$ Therefore, we aimed to obtain novel insight into the pathogenesis of SD by identifying hepatic microRNAs associated with the development of SD, and by studying the links between these microRNAs and tumour recurrence as well as survival.

\section{Materials and methods}

\section{Patients and sample selection}

A total of 40 patients who underwent partial hepatectomy for CRLM and had preoperative oxaliplatin-based chemotherapy at Maastricht University Medical Centre between Jan 2008 and Dec 2009 were included in the study. Plasma was collected after general anesthesia was induced, but before liver surgery. Non-tumour liver biopsies ( $>2 \mathrm{~cm}$ distance from the tumour) were taken before manipulation of the liver. The study was approved by the Medical Ethics Committee of Maastricht University Medical Centre and conducted according to the revised version of the Declaration of Helsinki. Written informed consent was obtained from each subject prior to study participation.

\section{Assessment of sinusoidal injury}

Formalin-fixed, paraffin-embedded archived samples of the non-tumour liver parenchyma were reviewed by two experienced liver pathologists (CV and AD). In case of dissent, final consensus was made after discussion. Morphology analysis was based on hematoxylin and eosin (H\&E), reticulin, and Masson Trichrome stained slides. Histopathology features were scored according to the classification of Rubbia-Brandt and colleagues. ${ }^{5}$ Based on these criteria, SD was semi-quantified from 0 to 3: 0, absent $(n=14) ; 1$, mild (centrilobular involvement limited to one third of the lobular surface, $\mathrm{n}=12$ ); 2 , moderate (centrilobular involvement extended to two thirds of the lobular surface, $n=11$ ); and 3, severe (the whole lobule involved, $n=3$ ).

\section{Microarray analyses}

A well-matched population of patients with grade 2-3 SD and patients without SD (grade $0)$ was selected for investigating microRNA expression. The matching parameters were: number of oxaliplatin cycles, cumulative oxaliplatin dose, sex, age, body mass index, and preoperative alanine transaminase and aspartate transaminase concentrations. Snap- 
frozen liver biopsy specimens from the 20 patients who could be matched were cut into small pieces, of which the largest dimension was less than $0.5 \mathrm{~cm}$. Samples were immediately soaked in pre-chilled RNAlater (Life Technologies, Bleiswijk, NL) and kept for at most 24 hours at $4^{\circ} \mathrm{C}$. RNAlater-stabilized liver tissue was homogenized in $700 \mu \mathrm{l}$ QIAzol Lysis Reagent (Qiagen, Venlo, NL) according to the manufacturer's protocol. RNA purification procedures were carried out with the miRNeasy Mini Kit (Qiagen, Venlo, NL) as instructed by the manufacturer. The quality of purified RNA was assessed using the Bioanalyser 2100 (Agilent Technologies, Amstelveen, NL). Samples with an RNA integrity number $(\mathrm{RIN})>6.0$ were used for cDNA preparation and hybridization.

With an input of 100ng RNA, cDNA targets were prepared by following the protocol for the microRNA microarray system with the microRNA complete labeling and hyb kit (Agilent, version 2.4, September 2011). MicroRNA expression profiling was performed using Agilent Sureprint G3 Human V19 microRNA 8 x 60K microarrays. The hybridization was performed according to the manufacturer's protocol; afterwards, the microarray slides were washed and scanned with a DNA microarray scanner (Agilent Technologies, Amstelveen, NL). The scanned images were converted into TXT files using the Feature Extraction Software v10.7.3.1 from Agilent Technologies, and were imported in an inhouse developed pipeline for quality control using R 2.15.2 (http://www.r-project.org).

Further downstream analysis was performed by means of MagiCMicroRna. ${ }^{17}$ Total microRNA signals were log2 transformed and quantile normalized. The MagiCMicroRna filtering procedure was based on subsets of experimental conditions (grade 2-3 versus grade 0 SD). Gene Expression Omnibus (GEO) number to be assigned.

\section{Quantitative PCR analysis of liver specimens}

To validate the results from the microarray, we performed a quantitative PCR analysis of the 20 samples analyzed by microarray as well as 8 additional samples of patients with SD grade 0,2 , or 3 that could not be matched for the above-mentioned parameters. Snap-frozen liver specimens were homogenized in Lysis/Binding Buffer provided by the mirVana miRNA Isolation Kit (Life technologies, Bleiswijk, NL). Total RNA was isolated according to the manufacturer's protocol. cDNA was synthesized from $1.7 \mu \mathrm{g}$ of total RNA using the miScript II RT Kit (Qiagen, Venlo, NL). The procedures were performed according to the manufacturer's protocol. Afterwards, quantitative PCR was performed with the SensiMix SYBR \& Fluorescein Mastermix (Bioline, Alphen aan den Rijn, NL) and the appropriate miScript Primer Assay (Qiagen) in an iQ5 iCycler (Bio-Rad, Veenendaal, $\mathrm{NL}$ ) according to the manufacturer's instructions. The quantitative PCR protocol was: $95^{\circ} \mathrm{C}$ for 15 minutes, followed by 40 cycles of $95^{\circ} \mathrm{C}$ for 10 seconds, $60^{\circ} \mathrm{C}$ for 20 seconds, and $70^{\circ} \mathrm{C}$ for 20 seconds. Relative miR-21 and miR-150 expression was calculated with 
LinregPCR software (version 2014.2, Heart Failure Research Centre, Amsterdam, NL) using miR-16 as a stably-expressed reference microRNA. Sequences of the applied PCR primers are listed in Supplemental Table S6.1 (supporting information).

\section{Quantitative PCR analysis of plasma specimens}

To explore the potential association between hepatic microRNA expression and microRNA concentration in systemic blood (plasma), we also performed quantitative PCR of EDTA treated plasma from the patients included for quantitative PCR analysis (one patient with unavailable plasma was excluded). Total RNA was isolated from $200 \mu \mathrm{l}$ plasma using the miRNeasy Serum/Plasma Kit (Qiagen, Venlo, NL), and subsequently eluted in $14 \mu \mathrm{l}$ RNase-free water according to the manufacturer's instructions. $9 \mu \mathrm{l}$ extracted RNA was used (per 20 $\mu$ l reaction) to synthesize cDNA using the miScript II RT Kit (Qiagen, Venlo, NL). The $20 \mu$-reaction mix was further diluted 11 times in nucleasefree water, and $5 \mu$ l of cDNA was used per quantitative PCR using SensiMix SYBR \& Fluorescein Mastermix (Bioline, Alphen aan den Rijn, NL) and an iQ5 iCycler (Bio-Rad, Veenendaal, NL). Data were normalized for isolation efficiency using a mix of 3 spiked-in synthetic Caenorhabditis elegans microRNAs, ${ }^{18}$ lacking sequence homology to human microRNAs. Oligonucleotides $\left(2 \mu \mathrm{l} ; 4.8 \times 10^{8}\right.$ copies/ $\left.\mu \mathrm{l}\right)$ were spiked into the samples during RNA isolation. Sequences of the applied quantitative PCR primers are listed in Supplemental Table S6.1 (supporting information).

\section{Overall survival and recurrence-free survival}

Overall survival was calculated from the date of partial hepatic resection to the date of decease or to the date of last follow-up when patients were not deceased. Recurrencefree survival was calculated from the date of partial hepatic resection to 1 ) the date of first radiological examination with diagnosis of a recurrent lesion or 2) the date of last radiological follow-up when recurrence had not occurred. Assessment of recurrence was based on computed tomography, positron emission tomography-computed tomography examinations, or magnetic resonance imaging, and combined with pathology reports or carcinoembryonic antigen test whenever available. Overall recurrence included not only liver recurrence, but also lung, primary colorectal site, and other types of recurrence.

\section{Statistical analyses}

We considered microRNAs differentially expressed when they fulfilled the combination of the following criteria: 1 , a $p$ value $<0.05$ determined by an unpaired moderated $T$-test using the Bioconductor's Limma package; ${ }^{19} 2$, absolute fold change (FC) $>1.2$ (in accordance with log2 ratio of $<-0.263$ or $>0.263$ ). 
All other statistical analyses were conducted using IBM SPSS software for Windows (version 20.0). For continuous variables $p$-values were calculated using Mann-Whitney $U$ test; and for categorical variables, Chi-square test with continuity correction or Fisher's exact test was used. Spearman correlation analysis was used to assess the correlation between parameters of interest. Survival curves were generated according to the Kaplan-Meier method in GraphPad Prism 6 for Windows (Version 6.05), and the $p$ values were computed using the Log-rank test. The median follow-up was calculated according to the reverse Kaplan-Meier technique. A $p$ value of less than 0.05 was considered statistically significant for all tests.

\section{Results}

\section{Patient characteristics}

Of the 40 patients assessed by pathologists, 28 displayed SD grade 0, 2, or 3 and were finally included in this study (SD grade 2-3 versus grade 0). General characteristics of these patients are summarized in Table 6.1. Of the 28 patients included, 20 patients could be matched for important parameters such as oxaliplatin exposure, sex, age, BMI, and liver injury markers, and were selected for subsequent microarray analyses. Group 1 $(n=10)$ consisted of patients with grade 2-3 SD. Group $2(n=10)$ consisted of patients without SD (grade 0). General characteristics of these patients are summarized in Table 6.2.

Reduced miR-21 and miR-150 expression levels in the liver of patients with severe SD

To investigate which microRNAs were differentially expressed in the liver of patients with and without SD, we performed microarray analysis using 20 prudently matched specimens. Surprisingly, not a single microRNA was significantly upregulated in the livers of patients with grade 2-3 SD as compared to patients without SD. Moreover, only two microRNAs were significantly downregulated in patients with SD grades 2-3 (Figure 6.1, $A$ and B): miR-21 and miR-150. Hepatic miR-21 expression was 1.34-fold lower in patients with SD ( $p=0.028)$, whereas miR-150 expression was reduced to a larger extent in this population (1.46-fold, $p=0.035$ ). 
Table 6.1 General characteristics of all included patients.

\begin{tabular}{|c|c|c|c|}
\hline & $\begin{array}{c}\text { Sinusoidal Dilatation } \\
\text { Absent } \\
(n=14)\end{array}$ & $\begin{array}{c}\text { Sinusoidal Dilatation } \\
\text { Grade } 2-3 \\
(n=14)\end{array}$ & $p$ value \\
\hline \multicolumn{4}{|l|}{ General characteristics } \\
\hline Sex (male) & 6 & 6 & 1.000 \\
\hline Age (years) & $64 \pm 3$ & $60 \pm 3$ & 0.329 \\
\hline $\mathrm{BMI}\left(\mathrm{kg} / \mathrm{m}^{2}\right)$ & $25.6 \pm 1.0$ & $25.5 \pm 0.8$ & 0.910 \\
\hline $\operatorname{ALT}(U / L)$ & $37 \pm 4$ & $29 \pm 4$ & 0.219 \\
\hline AST (U/L) & $32 \pm 4$ & $26 \pm 4$ & 0.410 \\
\hline \multicolumn{4}{|l|}{ Chemotherapy } \\
\hline Number of Oxaliplatin cycles & $6.1 \pm 0.6$ & $5.7 \pm 0.6$ & 0.667 \\
\hline Cumulative Oxaliplatin dose (mg) & $1345 \pm 121$ & $1362 \pm 128$ & 1.000 \\
\hline Bevacizumab treatment & 11 & 10 & 1.000 \\
\hline \multicolumn{4}{|l|}{ Other characteristics } \\
\hline Major hepatectomy & 5 & 6 & 1.000 \\
\hline Perioperative blood transfusion (yes) & 7 & 3 & 0.236 \\
\hline \multicolumn{4}{|l|}{ Morbidity } \\
\hline None & 6 & 7 & 1.000 \\
\hline Minor (Clavien-Dindo grade <3a ) & 3 & 5 & 0.678 \\
\hline Major (Clavien-Dindo grade $\geq 3 a$ ) & 5 & 2 & 0.385 \\
\hline Length of hospital stay (days) & $12 \pm 3$ & $12 \pm 4$ & 0.756 \\
\hline
\end{tabular}

SD, sinusoidal dilatation; BMI, body mass index; ALT, alanine aminotransferase; AST, aspartate aminotransferase; continuous variables are presented as mean (standard deviation), and $p$-values were calculated using Mann-Whitney $U$ test; categorical variable was tested using Pearson Chi-Square test with continuity correction.

Table 6.2 General characteristics of patients matched for microarray analyses.

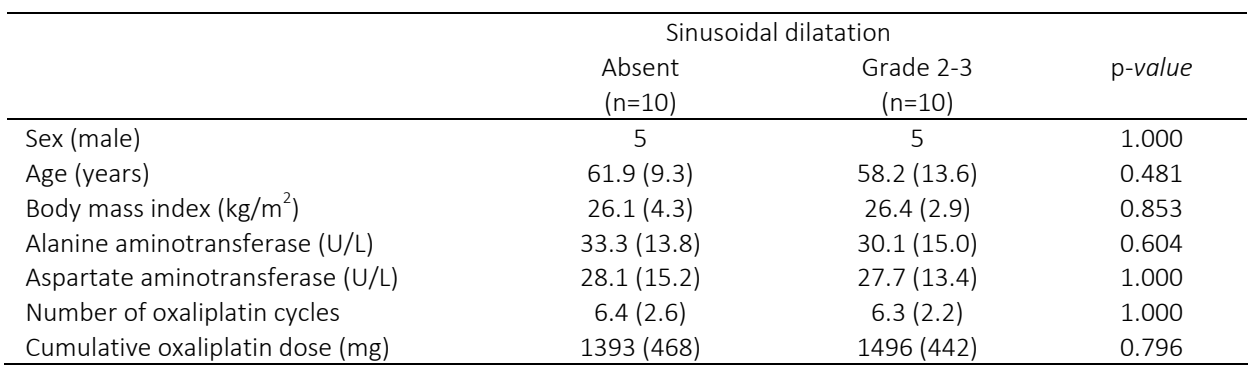

Continuous variables are presented as mean (standard deviation), and $p$-values were calculated using MannWhitney $U$ test; categorical variable was tested using Pearson Chi-Square test with continuity correction.

To validate these microarray data, we first performed quantitative PCR using liver specimens from the same set of patients. Again, miR-21 and miR-150 were found to be downregulated in the liver of patients with grade 2-3 SD, confirming the array data. The fold change of miR-21 was $1.34 \quad(p=0.015)$; the fold change of miR-150 was 1.39 $(p=0.011)$. Next, we further validated hepatic downregulation of miR-21 and miR-150 in grade 2-3 SD using the entire set of patients with and without SD who fulfilled the 
inclusion criteria $(n=28)$. In line with the microarray data, we found a significant downregulation of both miR-21 and miR-150 in patients with grade 2-3 SD. The fold changes were similar to those observed in the microarray experiment $(1.32, p=0.001$ and 1.34, $p=0.014$, respectively (Figure 6.1, C and D)).
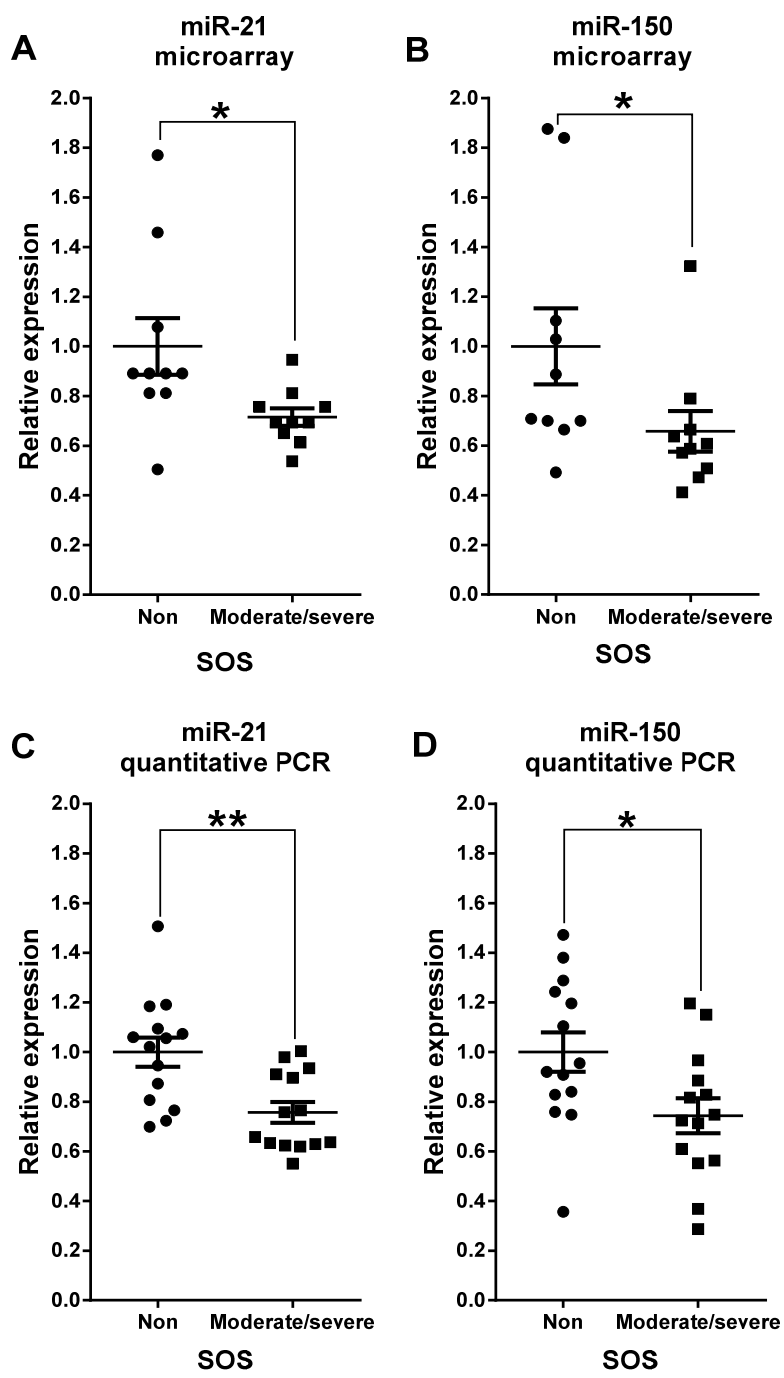

Figure 6.1 Reduced relative hepatic miR-21 and miR-150 expression in high grade sinusoidal dilatation patients.

$A$ and B: Compared with the non- sinusoidal dilatation group, both hepatic miR-21 (A) and miR150 (B) were downregulated in the severe sinusoidal dilatation group as assessed by microarray analysis $(n=20)$. C and D: Quantitative PCR analysis of a slightly bigger set of patients $(n=28)$ 


\section{Hepatic microRNA expression changes in SD are not reflected in plasma}

There is an increasing interest in the use of circulating microRNAs as biomarkers of disease. In view of the downregulation of miR-21 and miR-150 in the liver of patients with grade 2-3 SD, we next explored the possibility that these microRNA changes would also be detectable in plasma, offering a way to monitor SD development and/or progression during chemotherapy treatment. Both miR-21 and miR-150 were detectable in the plasma of patients either with or without SD. However, there were no significant differences in circulating levels of microRNA between these patients groups (Figure 6.2, $A$ and $B$ ). Moreover, whereas hepatic and plasma levels of miR-150 were correlated, this was not found for miR-21 (Figure 6.2, C and D).

\section{Low hepatic miR-21 and miR-150 expression is related to impaired} survival

MiR-21 and miR-150 have been demonstrated to play important roles in tumour cell proliferation, migration, and invasion, thereby affecting postoperative survival in colorectal cancer patients. ${ }^{12,20}$ In addition, patients with SD are known to display earlier recurrence and shorter overall survival after partial hepatectomy for colorectal liver metastases. ${ }^{9}$ We therefore next investigated the potential link between SD, miR-21 and miR-150 expression, and survival.

The median follow-up was 83 months for overall survival, 66 months for overall recurrence-free survival, and 61 months for liver recurrence-free survival. Of the 28 patients studied, 20 patients developed recurrence within the follow-up period; 1 patient underwent non-radical hepatic resection and was excluded from this analysis. The recurrence sites were liver $(n=9)$, lung $(n=5)$, skeleton $(n=3)$, primary colorectal site $(n=1)$, mediastinum $(n=1)$, and peritoneum $(n=1)$.

Surprisingly, SD was neither associated with impaired overall survival ( $p=0.711)$ nor with overall recurrence-free survival $(p=0.900)$ or liver recurrence-free survival $(p=0.971)$ in our patient group. However, when we defined $50^{\text {th }}$ percentile groups of high and low microRNA expression, we found that low expression of liver miR-21 was related to impaired overall survival ( $p=0.032$, Figure 6.3A) as well as shorter overall recurrence-free survival ( $p=0.010$, Figure 6.3B) and shorter liver recurrence-free survival ( $p=0.046$, Figure $6.3 C)$. Similarly, low hepatic miR-150 expression was related to impaired overall survival $(p=0.010$, Figure 6.3D), and reduced overall recurrence-free survival ( $p=0.025$, Figure 
$6.3 E$ ) as well as shorter liver recurrence-free survival ( $p=0.031$, Figure 6.3F). There was no correlation between miR-21 and miR-150 in plasma and overall survival or recurrence-free survival (data not shown).

A

miR-21 quantitative PCR

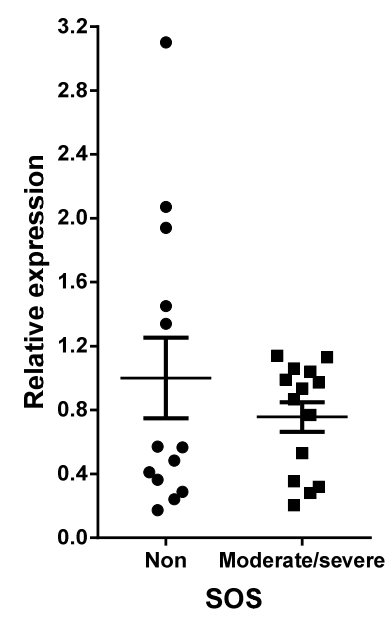

C Correlation of hepatic

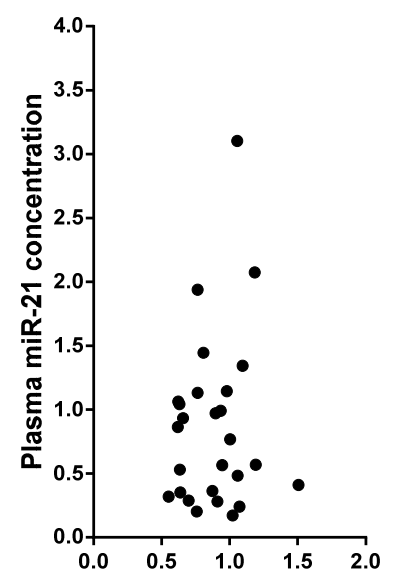

Hepatic miR-21 expression

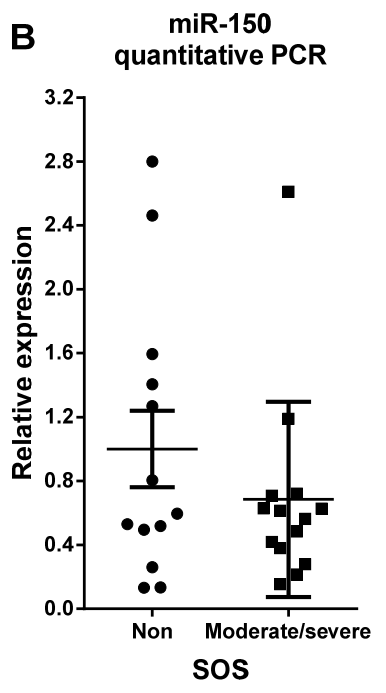

Correlation of hepatic
and plasma miR-150

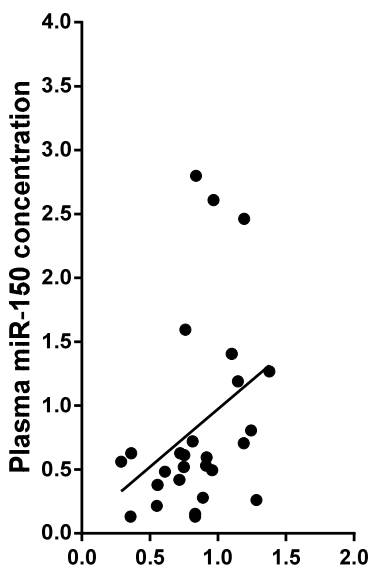

Hepatic miR-150 expression

Figure 6.2 Hepatic microRNA expression changes in sinusoidal dilatation are nit reflected in plasma.

$A$ and B: Similar plasma miR-21 (A) and miR-150 (B) concentrations in patients with and without sinusoidal dilatation $(\mathrm{n}=27), p=0.867$ and $p=0.519$, respectively. C: Hepatic miR-21 expression was not correlated with miR-21 plasma levels, $r=0.081, p=0.687$. D: Correlation between hepatic miR-150 expression and miR-150 plasma levels, $r=0.438, p=0.022$. 
A

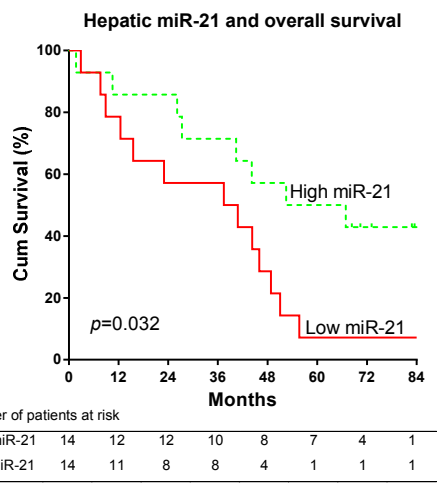

C Hepatic miR-21 and liver recurrence free survival

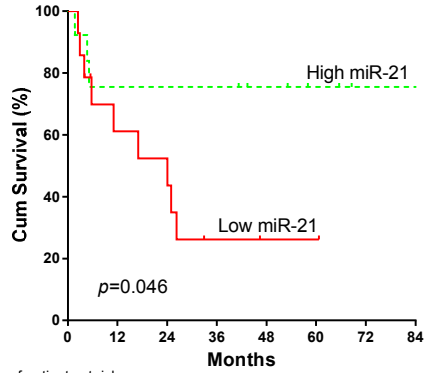

Number of patients at risk

\begin{tabular}{lllllllll}
\hline High miR-21 & 13 & 9 & 9 & 9 & 7 & 5 & 3 & 3
\end{tabular} \begin{tabular}{lllllllll} 
Low miR-21 & 14 & 7 & 6 & 2 & 1 & 1 & 0 & 0 \\
\hline
\end{tabular}

E Hepatic miR-150 and overall recurrence free survival

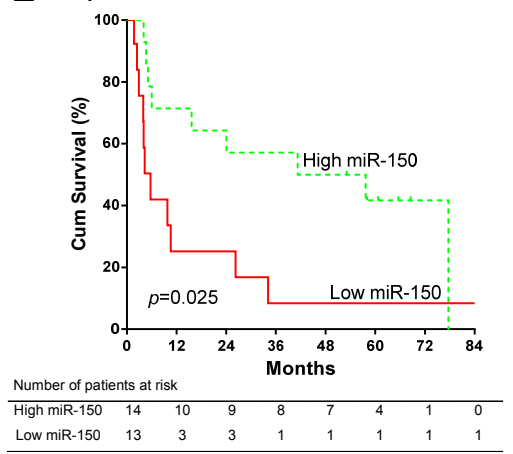

B Hepatic miR-21 and overall recurrence free survival
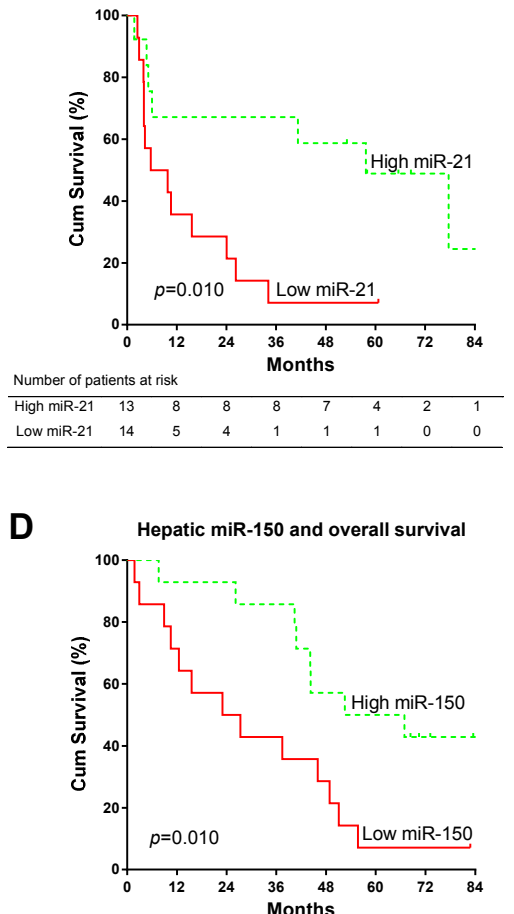
\begin{tabular}{lcccccccc} 
Number of patients at risk \\
\hline High miR-150 & 14 & 13 & 13 & 12 & 8 & 7 & 4 & 2 \\
Low miR-150 & 14 & 10 & 7 & 6 & 4 & 1 & 1 & 0
\end{tabular}

F Hepatic miR-150 and liver recurrence free survival

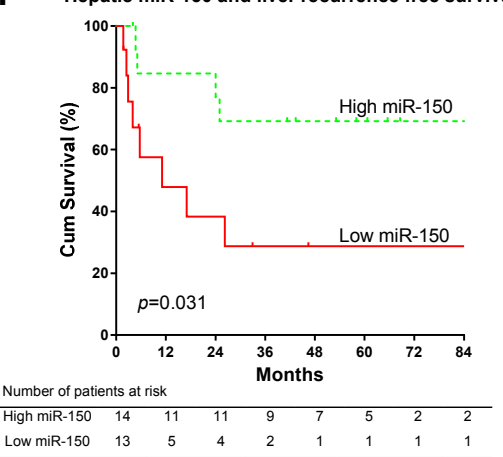

Figure 6.3 Low hepatic miR-21 and miR-150 expression are correlated with impaired long-term survival. A: Low hepatic miR-21 expression is associated with reduced overall survival. B: Patients with low hepatic miR-21 expression display reduced overall recurrence-free survival. C: Reduced liver recurrence-free survival in patients with low hepatic miR-21 expression. D: Low hepatic miR-150 expression is related to reduced overall survival. E: Patients with low hepatic miR-150 expression display reduced overall recurrence-free survival. F: Patients with low hepatic miR-150 expression display reduced liver recurrence-free survival. 


\section{Discussion}

SD is a serious complication in patients with CRLM treated with oxaliplatin-based chemotherapy. It impairs long term survival and negatively affects tumour response to chemotherapy treatment. ${ }^{6,7}$ The pathophysiological mechanisms responsible for the development of SD as well as its detrimental effects on patient outcome are still unclear. In the present study, we show that SD is characterized by reduced hepatic miR-21 and miR-150 expression, and that low expression of these microRNAs is associated with poorer survival.

Previous studies have implicated miR-150 in the context of colorectal cancer. For example, miR-150 expression in colorectal tissue was recently shown to diminish in parallel with increasing carcinogenesis. ${ }^{12}$ In line with our data, low miR-150 expression was associated with poor survival as well as a reduced response to adjuvant chemotherapy. ${ }^{12}$ Interestingly, whereas colorectal tumour displayed lower miR-150 expression than normal colorectal mucosa, ${ }^{11}$ patients with colorectal tumour with liver metastases had higher tumour miR-150 expression than those without liver metastases. ${ }^{21}$ Together with the fact that miR-150 is also expressed in other tissues such as adipose tissue, ${ }^{22}$ this may explain why we did not find reduced miR-150 levels in the plasma of patients with SD, who were characterized by lower hepatic miR-150 expression.

A key question is how reduced miR-150 expression may contribute to the development of SD, and how this might impact survival. With respect to survival, it is noteworthy that miR-150 has been suggested to function as tumour suppressor in colorectal cancer. For example, miR-150 appears to inhibit colorectal tumour cell migration and invasion by targeting MUC4. ${ }^{23}$ Furthermore, miR-150 has been suggested to induce colorectal tumour cell apoptosis and to reduce cell migration and invasion by inhibiting c-Myb, which is overexpressed in colon tumour and plays crucial roles in cell proliferation. ${ }^{20}$ In addition, miR-150 targets vascular endothelial growth factor (VEGF), ${ }^{24}$ a factor that is known to induce endothelial cell migration and mediate the formation of new blood vessels in tumour. Since VEGF has been found to be upregulated in oxaliplatin-related severe SD, ${ }^{10}$ the effect of miR-150 on VEGF may also be relevant in the context of SD. SD-associated VEGF upregulation is consistent with the downregulation of miR-150 that we observed in the liver of patients with SD, and suggests that low miR-150 levels may contribute to the observed increase in VEGF in SD. VEGF upregulation, in turn, has been suggested to increase the expression of matrix metalloproteinases that promote detachment of sinusoidal endothelial cells from the basement membrane and promote 
SD development. ${ }^{10}$ An additional potential mechanism by which miR-150 may affect SD relates to its suppressive effect on hepatic stellate cells activation. ${ }^{25}$ Hepatic stellate cells promote SD progression by depositing high amounts of collagen in the space of Disse's, ${ }^{5,10}$ a process that appears to be suppressed by the binding of miR-150 to collagen 4A4 and Sp1, a mediator of $\alpha-1(\mathrm{I})$ collagen expression. ${ }^{25}$ Thus, taken together, miR-150 downregulation may play a crucial role not only in promoting the development of SD but also in reducing tumour suppression, thereby potentially explaining the relation between miR-150 expression and impaired overall and recurrence-free survival that we observed.

In contrast to the reduced expression of miR-150 in colorectal cancer, miR-21 appears to be overexpressed in colorectal cancer cells. ${ }^{26}$ In addition, higher serum and plasma miR21 concentrations have been reported in colorectal cancer patients, ${ }^{15}$ and high colorectal tumour miR-21 expression has been associated with poorer prognosis in colorectal cancer patients. ${ }^{27}$ On the other hand, it was recently shown that reduced serum miR-21 levels are related to higher local colorectal cancer recurrence. ${ }^{14}$ This is in line with the decreased overall and recurrence-free survival that we observed in patients with low hepatic miR-21 expression.

Mechanistically, antisense inhibition of miR-21 has been shown to inhibit tumour cell migration and to sensitize tumour cells to anticancer agents, indicating that miR-21 plays an active role in colorectal tumour progression. ${ }^{28}$ Furthermore, miR-21 overexpression has been previously demonstrated to impair angiogenesis from endothelial cells by inhibiting RhoB. ${ }^{29}$ Importantly, gene expression profiling revealed that the angiogenesis pathway is upregulated in oxaliplatin-related SD. ${ }^{10}$ Thus, the low miR-21 expression we found in patients with SD may contribute to the increased expression of proteins related to angiogenesis. Unlike miR-150, miR-21 activates hepatic stellate cells via PTEN/Akt signaling, and stimulates matrix metalloproteinases. ${ }^{30}$ The reduced expression of miR-21 in severe SD may therefore partly balance the detrimental effects of miR-150 on hepatic stellate cell activation and collagen deposition.

The fact that our study could not confirm the previously reported ${ }^{9}$ association between SD and impaired survival may be due to selection bias in our relatively small study cohort. Indeed, we mainly controlled for factors that we expected to affect gene expression, but that are not necessarily related to prognostic factors of survival.

To our knowledge, this is the first study to investigate the microRNA expression profile of oxaliplatin-related SD. Both identified downregulated microRNAs may not only play 
important roles in SD development, but also impact overall survival. Further studies focused on the genes targeted by miR-21 and miR-150 will likely contribute to revealing the mechanisms of oxaliplatin-related SD and could support clinicians to inhibit the development of SD, improving postoperative outcomes. 


\section{References}

1. Ferlay J, Soerjomataram I, Dikshit R, Eser S, Mathers C, Rebelo M, et al. Cancer incidence and mortality worldwide: sources, methods and major patterns in GLOBOCAN 2012. Int J Cancer 2015; 136(5):E359-86.

2. Kanas GP, Taylor A, Primrose JN, Langeberg WJ, Kelsh MA, Mowat FS, et al. Survival after liver resection in metastatic colorectal cancer: review and meta-analysis of prognostic factors. Clin Epidemiol 2012; 4:283301.

3. Nordlinger B, Van Cutsem E, Rougier P, Kohne CH, Ychou M, Sobrero A, et al. Does chemotherapy prior to liver resection increase the potential for cure in patients with metastatic colorectal cancer? A report from the European Colorectal Metastases Treatment Group. Eur J Cancer 2007; 43(14):2037-45.

4. Robinson SM, Wilson CH, Burt AD, Manas DM, White SA. Chemotherapy-associated liver injury in patients with colorectal liver metastases: a systematic review and meta-analysis. Ann Surg Oncol 2012; 19(13):4287-99.

5. Rubbia-Brandt L, Audard V, Sartoretti P, Roth AD, Brezault C, Le Charpentier M, et al. Severe hepatic sinusoidal obstruction associated with oxaliplatin-based chemotherapy in patients with metastatic colorectal cancer. Ann Oncol 2004; 15(3):460-6.

6. Vreuls CP, Van Den Broek MA, Winstanley A, Koek GH, Wisse E, Dejong $\mathrm{CH}$, et al. Hepatic sinusoidal obstruction syndrome (SOS) reduces the effect of oxaliplatin in colorectal liver metastases. Histopathology 2012; 61(2):314-8.

7. Vigano L, Capussotti L, De Rosa G, De Saussure WO, Mentha G, Rubbia-Brandt L. Liver resection for colorectal metastases after chemotherapy: impact of chemotherapy-related liver injuries, pathological tumor response, and micrometastases on long-term survival. Ann Surg, Vol. 258, 2013. pp. 731-40; discussion 741-2.

8. Nakano H, Oussoultzoglou E, Rosso E, Casnedi S, Chenard-Neu MP, Dufour P, et al. Sinusoidal injury increases morbidity after major hepatectomy in patients with colorectal liver metastases receiving preoperative chemotherapy. Ann Surg 2008; 247(1):118-24.

9. Tamandl D, Klinger M, Eipeldauer S, Herberger B, Kaczirek K, Gruenberger B, et al. Sinusoidal obstruction syndrome impairs long-term outcome of colorectal liver metastases treated with resection after neoadjuvant chemotherapy. Ann Surg Oncol 2011; 18(2):421-30.

10. Rubbia-Brandt L, Tauzin S, Brezault C, Delucinge-Vivier C, Descombes P, Dousset B, et al. Gene expression profiling provides insights into pathways of oxaliplatin-related sinusoidal obstruction syndrome in humans. Mol Cancer Ther 2011; 10(4):687-96.

11. Pizzini S, Bisognin A, Mandruzzato S, Biasiolo M, Facciolli A, Perilli L, et al. Impact of microRNAs on regulatory networks and pathways in human colorectal carcinogenesis and development of metastasis. BMC Genomics 2013; 14:589.

12. Ma Y, Zhang $P$, Wang F, Zhang H, Yang J, Peng J, et al. miR-150 as a potential biomarker associated with prognosis and therapeutic outcome in colorectal cancer. Gut 2012; 61(10):1447-53.

13. Krauskopf J, Caiment F, Claessen SM, Johnson KJ, Warner RL, Schomaker SJ, et al. Application of highthroughput sequencing to circulating microRNAs reveals novel biomarkers for drug-induced liver injury. Toxicol Sci 2015; 143(2):268-76.

14. Menendez P, Padilla D, Villarejo P, Palomino T, Nieto P, Menendez JM, et al. Prognostic implications of serum microRNA-21 in colorectal cancer. J Surg Oncol 2013; 108(6):369-73.

15. Toiyama Y, Takahashi M, Hur K, Nagasaka T, Tanaka K, Inoue Y, et al. Serum miR-21 as a diagnostic and prognostic biomarker in colorectal cancer. J Natl Cancer Inst 2013; 105(12):849-59.

16. Janssen HL, Reesink HW, Lawitz EJ, Zeuzem S, Rodriguez-Torres M, Patel K, et al. Treatment of HCV infection by targeting microRNA. N Engl J Med 2013; 368(18):1685-94.

17. Coonen M, Theunissen DH, Kleinjans JC, Jennen DG. MagiCMicroRna: a web implementation of AgiMicroRna using shiny. Source Code Biol Med 2015; 10:4.

18. Corsten MF, Dennert R, Jochems S, Kuznetsova T, Devaux Y, Hofstra L, et al. Circulating MicroRNA-208b and MicroRNA-499 reflect myocardial damage in cardiovascular disease. Circ Cardiovasc Genet 2010; 3(6):499-506.

19. Smyth G. Limma: linear models for microarray data. In: Gentleman R, Carey V, Huber W, Irizarry R, Dudoit S, eds. Bioinformatics and Computational Biology Solutions Using R and Bioconductor. New York: Springer; 2005:pp. 397-420. 
20. Feng J, Yang $\mathrm{Y}$, Zhang $\mathrm{P}, \mathrm{Wang} \mathrm{F}, \mathrm{Ma} \mathrm{Y}$, Qin $\mathrm{H}$, et al. miR-150 functions as a tumour suppressor in human colorectal cancer by targeting c-Myb. J Cell Mol Med 2014; 18(10):2125-34.

21. Lin M, Chen W, Huang J, Gao H, Ye Y, Song Z, et al. MicroRNA expression profiles in human colorectal cancers with liver metastases. Oncol Rep 2011; 25(3):739-47.

22. van de Bunt M, Gaulton KJ, Parts L, Moran I, Johnson PR, Lindgren CM, et al. The miRNA profile of human pancreatic islets and beta-cells and relationship to type 2 diabetes pathogenesis. PLoS One 2013; 8(1):e55272.

23. Wang WH, Chen J, Zhao F, Zhang BR, Yu HS, Jin HY, et al. MiR-150-5p suppresses colorectal cancer cell migration and invasion through targeting MUC4. Asian Pac J Cancer Prev 2014; 15(15):6269-73.

24. Yu ZY, Bai YN, Luo LX, Wu H, Zeng Y. Expression of microRNA-150 targeting vascular endothelial growth factor-A is downregulated under hypoxia during liver regeneration. Mol Med Rep 2013; 8(1):287-93.

25. Zheng J, Lin Z, Dong P, Lu Z, Gao S, Chen X, et al. Activation of hepatic stellate cells is suppressed by microRNA-150. Int J Mol Med 2013; 32(1):17-24.

26. Gattolliat $\mathrm{CH}$, Uguen $\mathrm{A}$, Pesson $\mathrm{M}$, Trillet $\mathrm{K}$, Simon $\mathrm{B}$, Doucet $\mathrm{L}$, et al. MicroRNA and targeted mRNA expression profiling analysis in human colorectal adenomas and adenocarcinomas. Eur J Cancer 2015; 51(3):409-20.

27. Slaby O, Svoboda M, Fabian P, Smerdova T, Knoflickova D, Bednarikova M, et al. Altered expression of miR-21, miR-31, miR-143 and miR-145 is related to clinicopathologic features of colorectal cancer. Oncology 2007; 72(5-6):397-402.

28. Zhao Y, Zhao L, Ischenko I, Bao Q, Schwarz B, Niess H, et al. Antisense inhibition of microRNA-21 and microRNA-221 in tumor-initiating stem-like cells modulates tumorigenesis, metastasis, and chemotherapy resistance in pancreatic cancer. Journal [serial online]. 2015. Accessed Feb 3.

29. Connolly EC, Van Doorslaer K, Rogler LE, Rogler CE. Overexpression of miR-21 promotes an in vitro metastatic phenotype by targeting the tumor suppressor RHOB. Mol Cancer Res 2010; 8(5):691-700.

30. Zhao J, Tang N, Wu K, Dai W, Ye C, Shi J, et al. MiR-21 simultaneously regulates ERK1 signaling in HSC activation and hepatocyte EMT in hepatic fibrosis. PLoS One 2014; 9(10):e108005. 


\section{Supplemental tables}

Table S6.1 Primers for quantitative PCR.

\begin{tabular}{lll}
\hline Species & Name of primer & Sequence \\
\hline Human & Human miR-150 forward & 5' - TCTCCCAACCCTTGTACCAGTG \\
& Human miR-21 forward & 5' - TAGCTTATCAGACTGATGTTGA \\
& Human miR-16 forward & 5' - TAGCAGCACGTAAATATTGGCG \\
Caenorhabditis elegans & Cel-miR-39 forward & 5' - TCACCGGGTGTAAATCAGCTTG \\
& Cel-miR-54 forward & 5' - TACCCGTAATCTTCATAATCCGAG \\
& Cel-miR-238 forward & 5' - TTTGTACTCCGATGCCATTCAGA \\
\hline
\end{tabular}




\section{Chapter}

Hepatic complement activation is associated with liver injury in patients with colorectal liver metastases

Junfang Zhao, Sander S. Rensen, Celien P.H. Vreuls, Ann Driessen, Maartje A.J. van den Broek, Cornelis H.C. Dejong, Steven W.M. Olde Damink 


\section{Abstract}

\section{Background}

Preoperative systemic chemotherapeutic treatment for colorectal liver metastases frequently induces chemotherapy-associated liver injury (CALI). CALI encompasses liver phenotypes such as sinusoidal dilatation, nodular transformation, hepatocyte steatosis, and lobular inflammation. Since complement factors are known to be involved in these hepatic pathophysiologies, we investigated the link between complement activation and CALI.

\section{Methods}

50 consecutive patients who underwent partial hepatectomy for colorectal liver metastases (CRLM) were included. Sinusoidal dilatation and nodularity were assessed using formalin-fixed non-tumour liver samples. C3c, C5a, and terminal complement complex (TCC) concentrations were determined in liver tissue lysates by ELISA to investigate complement activation; neutrophil infiltration was assessed by myeloperoxidase staining and subsequently quantified as a measure of lobular inflammation; hepatic triglyceride concentration was analyzed to quantify steatosis.

\section{Results}

Hepatic $\mathrm{C} 3 \mathrm{c}$ and $\mathrm{C} 5 \mathrm{a}$ were both detectable, and $\mathrm{C} 3 \mathrm{c}$ concentrations strongly correlated with $\mathrm{C} 5 \mathrm{a}$ concentrations $(r=0.684, p<0.0001)$. C3c concentration was similar in patients with or without sinusoidal dilatation $(13.6 \pm 1.6$ vs. $13.5 \pm 1.2 \mathrm{ng} / \mathrm{ml}, \mathrm{p}=0.952)$ and in patients with or without nodular transformation $(14.2 \pm 3.2 \mathrm{vs} .13 .4 \pm 1.1 \mathrm{ng} / \mathrm{ml}, \mathrm{p}=0.750)$. In contrast, hepatic C5a levels were increased in patients with nodular transformation $(13.9 \pm 4.3 \mathrm{ng} / \mathrm{ml}$ vs. $7.4 \pm 1.1 \mathrm{ng} / \mathrm{ml}, \mathrm{p}=0.041)$, but not in those with sinusoidal dilatation $(10.4 \pm 2.1 \mathrm{ng} / \mathrm{ml}$ vs. $6.7 \pm 0.9 \mathrm{ng} / \mathrm{ml}, \mathrm{p}=0.220)$. Hepatic concentrations of $\mathrm{C} 3 \mathrm{c}$ and $\mathrm{C} 5 \mathrm{a}$ correlated with hepatic neutrophil infiltration $(r=0.373, p=0.008 ; r=0.485, p=0.0004$, respectively), but not with triglyceride concentrations.

\section{Conclusions}

The complement system appears to be activated in the livers of CLRM patients with hepatic nodular transformation and lobular inflammation. This warrants investigation of the potential effect of complement inhibition on the development of CALI. 


\section{Introduction}

Over the past decades, patients with colorectal liver metastases (CRLM) have been commonly treated with combined chemotherapeutic agents such as irinotecan and oxaliplatin. This strategy increased tumour response rates considerably from $24 \%$ to up to $70 \%$, compared to single treatment with 5-fluorouracil. ${ }^{1,2}$ However, neoadjuvant administration of these agents frequently has harmful side-effects, referred to as chemotherapy-associated liver injury (CALI). For instance, oxaliplatin-based treatments increase the occurrence of sinusoidal dilatation ${ }^{3}$ and the risk of nodular regenerative hyperplasia $(\mathrm{NRH}) .^{3-5}$ Irinotecan-based regimens appear to be related to the development of steatohepatitis. ${ }^{6}$

Pathophysiological processes leading to CALI include the activation of the acute phase response, ${ }^{7}$ which is characterized by increased secretion of complement factors by the liver. As a pivotal element of the innate immune system, the complement system mediates the initiation of inflammatory reactions, the clearance of immune complexes, and the regulation of the immune response to tissue injury. ${ }^{8}$ Activation of the central complement factor C3 results in the formation of C3a, a chemokine that recruits neutrophils into the liver and participates in the development of nonalcoholic steatohepatitis. ${ }^{9}$ Next, C3a is rapidly cleaved by carboxypeptidases to generate acylation stimulating protein (ASP), a peptide which plays a role in triglyceride metabolism and has been shown to be increased in nonalcoholic steatohepatitis. ${ }^{10,11}$

Sustained activation of C3 can result in the formation of C5 convertase, which cleaves complement factor $\mathrm{C} 5$ into $\mathrm{C} 5 \mathrm{~b}$ and $\mathrm{C} 5$ a. Similar to $\mathrm{C} 3 \mathrm{a}, \mathrm{C} 5 \mathrm{a}$ also promotes inflammation by attracting neutrophils. ${ }^{12}$ Moreover, it has recently been shown that $\mathrm{C} 5 \mathrm{a}$ stimulates hepatic stellate cells and plays a causal role in the regulation of liver fibrosis in various liver diseases. ${ }^{13-15}$ In addition, C5a has been shown to play a role in the early stages of liver regeneration after partial hepatectomy. ${ }^{16,17}$

Given the involvement of complement factors in these pathophysiological processes that also occur in CALI, we hypothesized that complement activation could play a role in patients undergoing partial hepatectomy for CRLM. 


\section{Materials and methods}

\section{Patients and sample selection}

Consecutive patients who underwent partial hepatectomy for CRLM at the Maastricht University Medical Centre between January 2008 and December 2009 were included in this study. Exclusion criteria were: a) patients who underwent preoperative portal venous embolization, since this procedure induces hepatic ischemia which probably interacts with sinusoidal changes related to chemotherapy; b) patients who were diagnosed with autoimmune liver disease, type $\mathrm{B}$ or $\mathrm{C}$ hepatic virus infection; c) patients with cirrhosis. Non-tumour liver specimens at a distance of more than $2 \mathrm{~cm}$ from the tumour were collected by two approaches: a) samples collected after opening the abdomen and before touching or manipulating the intestines or liver to prevent artificial influx of inflammatory cells ${ }^{18}$ were immediately snap-frozen in liquid nitrogen and stored at $-80^{\circ} \mathrm{C}$; b) Once the tumour was resected, additional non-tumour liver samples (at a distance of more than $2 \mathrm{~cm}$ from the tumour) were collected, fixed in $4 \%$ buffered formaldehyde, and embedded in paraffin. Frozen samples were used to quantify $\mathrm{C} 3 \mathrm{c}$, C5a, TCC, and triglyceride concentrations, and to stain neutrophils. Formalin-fixed paraffin-embedded samples were used to assess liver injuries (i.e. sinusoidal dilatation and nodular transformation). This study was approved by the Medical Ethics Committee of Maastricht University Medical Centre and conducted according to the revised version of the Declaration of Helsinki. Written informed consent was obtained from each subject prior to study participation.

\section{Assessment of sinusoidal dilatation and nodular transformation}

Morphological assessment was performed based on hematoxylin and eosin (H\&E), Masson's Trichrome, and reticulin stains as previously described. ${ }^{19}$ All specimens were independently reviewed by two experienced liver pathologists (C.V. and A.D.) to assess sinusoidal dilatation and nodular transformation in non-tumour liver using paraffin embedded tissue. Severity of sinusoidal dilatation was semi-quantified according to the classification of Rubbia-Brandt et al. as follows: 0, absent; 1, mild (centrilobular involvement limited to one-third of the lobular area); 2, moderate (centrilobular involvement extending in two-thirds of the lobular area); 3, severe (complete lobular involvement or centrilobular involvement extending to adjacent lobules with bridging congestion. ${ }^{3}$ Nodular transformation was assessed according to Wanless as follows: 0 , absent; 1 , mild (focal occasionally distinct nodular hyperplasia on reticulin staining but indistinct on H\&E staining); 2 , moderate (focal distinct nodular hyperplasia apparent on H\&E staining, clearly highlighted on reticulin staining); 3 , severe (diffuse nodular 
hyperplasia, distinct in most areas on H\&E staining and highlighted on reticulin staining, corresponding to nodular regenerative hyperplasia). ${ }^{20}$ Afterwards, patients were divided into two groups: those without liver injury (absent) versus those with liver injury (mild, moderate, or severe).

\section{Quantification of hepatic C3c, C5a, and TCC concentration}

Liver tissue lysates were made by homogenizing frozen liver tissue in ice-cold lysis buffer ( $\mathrm{pH}$ 7.4), which contained $30 \mathrm{mM}$ Tris $\cdot \mathrm{HCl}, 150 \mathrm{mM} \mathrm{NaCl}, 0.5 \%$ Triton X-100, 1\% (v/v) SDS, $100 \mathrm{mM}$ EDTA, $10 \mathrm{mM} \mathrm{NaF}$, and $1 \mathrm{mM} \mathrm{Na}_{3} \mathrm{VO}_{4}$. Hepatic concentrations of C3c, C5a, and terminal complement complex (TCC) were measured using human $\mathrm{C} 3 \mathrm{C}, \mathrm{C} 5 \mathrm{a}$, and TCC ELISA kits (Hycult Biotech, Uden, NL) according to the manufacturer's protocol. The final concentration was determined by referencing to the total protein concentration in the lysate as determined by the bicinchoninic acid (BCA) protein assay kit (ThermoFisher Scientific, Landsmeer, NL) according to the manufacturer's protocol.

\section{Quantification of liver triglyceride levels}

To quantify liver fat content, we measured liver triglyceride concentrations in tissue lysates using the triglyceride colorimetric assay kit (Cayman chemical, Uden, NL). The procedures were performed according to the manufacturer's protocol. The final concentration of liver triglyceride was determined by referencing to the total protein concentration.

\section{Immunohistochemistry}

Immunohistochemical staining was performed as previously described. ${ }^{20}$ Unmanipulated frozen non-tumour liver tissue was cut into 4- $\mu$ m-thick sections. Endogenous peroxidase activity was blocked by incubation in $0.6 \% \mathrm{H}_{2} \mathrm{O}_{2}$ for 15 minutes. After blocking with $5 \%$ bovine serum albumin, primary polyclonal rabbit antibody recognizing human myeloperoxidase (dilution 1:500; Dako, Heverlee, Belgium) was applied for 1 hour. Biotinylated swine-anti-rabbit IgG (dilution 1:500; Dako) was applied as secondary antibody for 1 hour, and followed by application of avidin-biotinhorseradish peroxidase system (dilution 1:50; DAKO). 3-Amino-9-ethylcarbazole was used as chromogen. Tissue sections were counterstained with Mayer's hematoxylin. Neutrophil numbers were quantified by photographing five randomly selected parts of myeloperoxidase-stained sections at 100 x magnifications followed by manual counting of stained cells. 


\section{Statistical analysis}

All statistical analyses were conducted using Graphpad Prism for Windows (version 6.05). The Student's t-test was used for continuous variables. For categorical variables, the Pearson Chi-square test with continuity correction was applied, or the Fisher's exact test when any of the expected values was smaller than five. Pearson product-moment correlation coefficient was computed and scatterplots were drawn to assess the relationship between two parameters of interest. A $p$ value $\leq 0.05$ was considered significant for all analyses.

\section{Results}

\section{Patient characteristics}

A total of 50 patients were included. Oxaliplatin, in combination with or without bevacizumab, was the most commonly administered chemotherapeutic agent (41/50). Details of the applied chemotherapy and general characteristics of the patients are reported in Table 7.1. Pathology examination of the non-tumour tissue revealed that 32 (64\%) patients had sinusoidal dilatation, and 13 (26\%) patients displayed nodular transformation. Patients with sinusoidal dilatation showed a higher prevalence of nodular transformation than those without sinusoidal dilatation $(12 / 32$ vs. $1 / 18$, $\mathrm{p}=0.024)$.

Table 7.1 General characteristics of included patients.

\begin{tabular}{lcccccc}
\hline & \multicolumn{3}{c}{ Sinusoidal dilatation } & \multicolumn{3}{c}{ Nodular transformation } \\
& None & Present & $p$ & None & Present & $p$ \\
& $(n=18)$ & $(n=32)$ & & $(n=37)$ & $(n=13)$ & \\
\hline Sex (male) & $9(50 \%)$ & $18(56 \%)$ & $0.897 \dagger$ & $19(51 \%)$ & $8(62 \%)$ & $0.756 \dagger$ \\
Age (years) & $65(9)$ & $62(11)$ & 0.497 & $63(10)$ & $63(12)$ & 0.986 \\
BMI (kg/m $\left.{ }^{2}\right)$ & $26.3(3.7)$ & $25.8(3.1)$ & 0.606 & $25.7(3.4)$ & $26.9(3.0)$ & 0.241 \\
AST (U/L) & $29(14)$ & $25(11)$ & 0.235 & $26(13)$ & $28(11)$ & 0.611 \\
ALT (U/L) & $33(15)$ & $27(11)$ & 0.107 & $30(13)$ & $27(13)$ & 0.531 \\
Oxaliplatin & $14(78 \%)$ & $25(78 \%)$ & $1.000 \ddagger$ & $28(76 \%)$ & $11(85 \%)$ & $0.808 \ddagger$ \\
Oxaliplatin cycles & $4.8(3.4)$ & $4.2(2.9)$ & 0.493 & $4.0(3.0)$ & $5.5(3.1)$ & 0.137 \\
Irinotecan & $1(6 \%)$ & $4(13 \%)$ & $0.802 \ddagger$ & $3(8 \%)$ & $2(15 \%)$ & $0.778 \ddagger$ \\
Irinotecan cycles & $0.3(1.4)$ & $0.6(1.9)$ & 0.614 & $0.6(2.0)$ & $0.3(0.9)$ & 0.646 \\
Capecitabine & $14(78 \%)$ & $27(84 \%)$ & $0.826 \ddagger$ & $30(81 \%)$ & $11(85 \%)$ & $1.000 \ddagger$ \\
Capecitabine cycles & $4.6(3.0)$ & $4.8(3.2)$ & 0.755 & $4.3(2.7)$ & $6.1(3.8)$ & 0.069 \\
Bevacizumab & $10(56 \%)$ & $20(63 \%)$ & $0.857 \dagger$ & $22(60 \%)$ & $8(62 \%)$ & $1.000 \dagger$ \\
Nodular transformation (present) & $1(6 \%)$ & $12(38 \%)$ & $0.024 \ddagger$ & n.a. & n.a. & n.a. \\
\hline
\end{tabular}

BMI, body mass index; AST, aspartate transaminase; ALT, alanine transaminase; n.a., not applicable; continuous data are shown as mean (standard deviation), and tested by Student's t test; †Pearson Chi-square test, with continuity correction; ¥Fisher's exact test 


\section{Complement activation in the liver of patients with CRLM}

$\mathrm{C} 3 \mathrm{c}$ has been shown to be a stable complement activation biomarker that is generated through cleavage of $\mathrm{C} 3{ }^{22}$ Hepatic $\mathrm{C} 3 \mathrm{c}$ and $\mathrm{C} 5$ a were detectable in all patients, indicating there was complement activation in the non-tumour liver parenchyma. The mean concentrations of $\mathrm{C} 3 \mathrm{c}$ and $\mathrm{C} 5 \mathrm{a}$ were $13.6 \mathrm{ng} / \mathrm{ml}$ and $9.1 \mathrm{ng} / \mathrm{ml}$, respectively. As expected, C3c and C5a concentrations showed a strong, positive correlation ( $r=0.684$, $p<0.0001$, Figure 7.1), indicative of sustained complement activation. However, hepatic TCC concentrations were too low to be detected.

\section{Hepatic C3c and C5a}

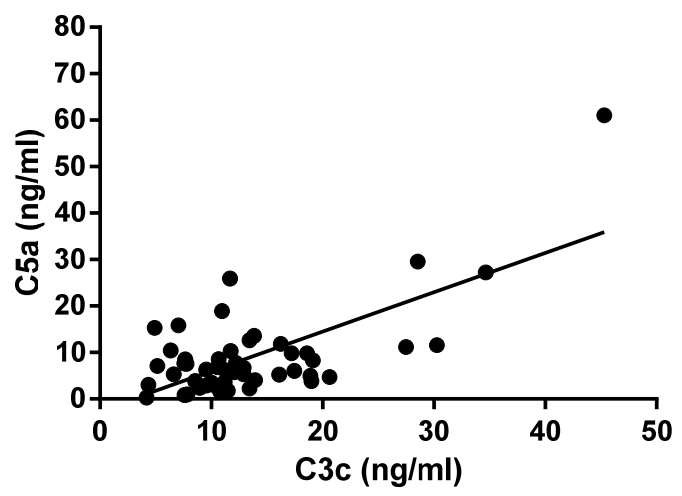

Figure 7.1 Correlation between hepatic C3c and C5a concentration.

Pearson correlation analyses showed a positive correlation between hepatic concentrations of C3c and C5a ( $r=0.684, n=50, p<0.0001)$

Increased hepatic C5a levels in patients with nodular transformation

Considering that oxaliplatin treatment is a risk factor for sinusoidal dilatation and nodular transformation, ${ }^{3,5}$ pathophysiologic processes underlying these hepatic parenchymal changes might be similar. Therefore, we next investigated a potential relationship between complement activation and these pathologies by comparing hepatic $\mathrm{C} 3 \mathrm{C}$ and $\mathrm{C} 5$ a concentrations in patients with and without sinusoidal dilatation or nodular transformation.

Neither C3c levels $(13.6 \pm 1.6 \mathrm{ng} / \mathrm{ml}$ vs. $13.5 \pm 1.2 \mathrm{ng} / \mathrm{ml}, \mathrm{p}=0.952$; Figure 7.2A) nor concentrations of C5a $(10.4 \pm 2.1 \mathrm{ng} / \mathrm{ml}$ vs. $6.7 \pm 0.9 \mathrm{ng} / \mathrm{ml}, \mathrm{p}=0.220$; Figure $7.2 \mathrm{~B})$ were significantly different between patients with and without sinusoidal dilatation. Although C3c levels were not significantly different between patients with and without nodular transformation $(14.2 \pm 3.2 \mathrm{ng} / \mathrm{ml}$ vs. $13.4 \pm 1.1 \mathrm{ng} / \mathrm{ml}, \mathrm{p}=0.750$; Figure $7.2 \mathrm{C}), \mathrm{C5a}$ 
concentrations were increased in patients with these lesions $(13.9 \pm 4.3 \mathrm{ng} / \mathrm{ml}$ vs. $7.4 \pm 1.1$ $\mathrm{ng} / \mathrm{ml}, \mathrm{p}=0.041$; Figure 7.2D).

A

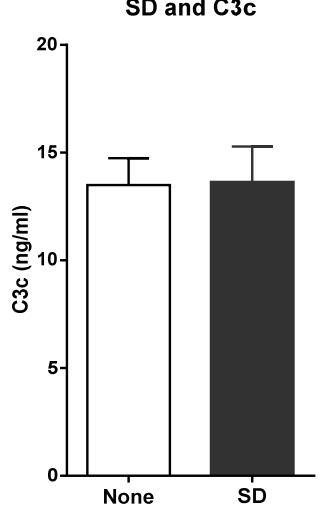

C

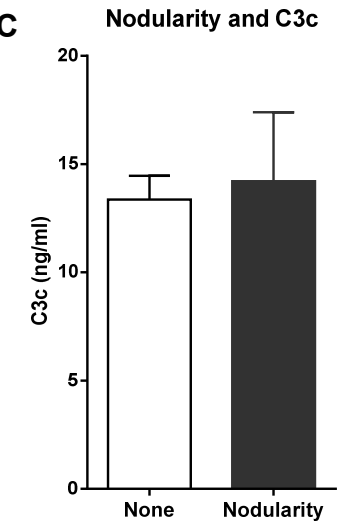

B

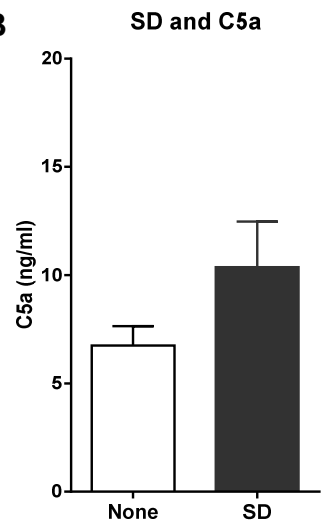

D

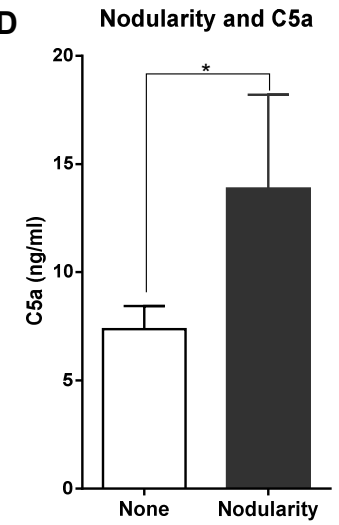

Figure 7.2 Hepatic C5a levels are not significantly increased in patients with sinusoidal dilatation, but increased in those with nodular transformation.

A and B: Hepatic C3C (A) and C5a (B) concentrations are not significantly different in patients with and without sinusoidal dilatation, $p=0.952$ and $p=0.220$, respectively. $C$ : Similar hepatic $C 3 c$ concentration in patients with and without nodular transformation, $p=0.750$. D: Increased hepatic C5a concentrations in patients with nodular transformation, $p=0.041$. SD, sinusoidal dilatation; Error bar represents mean with standard error of mean

\section{Hepatic C3c and C5a concentrations correlate with hepatic neutrophil infiltration}

Next, we investigated potential links between complement activation and hepatic steatosis as well as inflammation, two important elements of CALI. Hepatic triglyceride 
concentrations were not correlated with either hepatic $C 3 c(r=0.070, p=0.627$; Figure $7.3 \mathrm{~A}$ ) or $\mathrm{C} 5 \mathrm{a}$ levels $(r=-0.173, p=0.229$; Figure $7.3 \mathrm{~B}$ ). In contrast, correlations were found between hepatic $\mathrm{C} 3 \mathrm{c}$ concentration and neutrophil infiltration ( $r=0.373, p=0.008$; Figure $7.3 C$ ), as well as between hepatic C5a concentration and neutrophil infiltration ( $r=0.485$, $p=0.0004$; Figure 7.3D). These data suggest that complement activation in subjects with $\mathrm{CALI}$ is related to hepatic inflammation independent of steatosis.

A
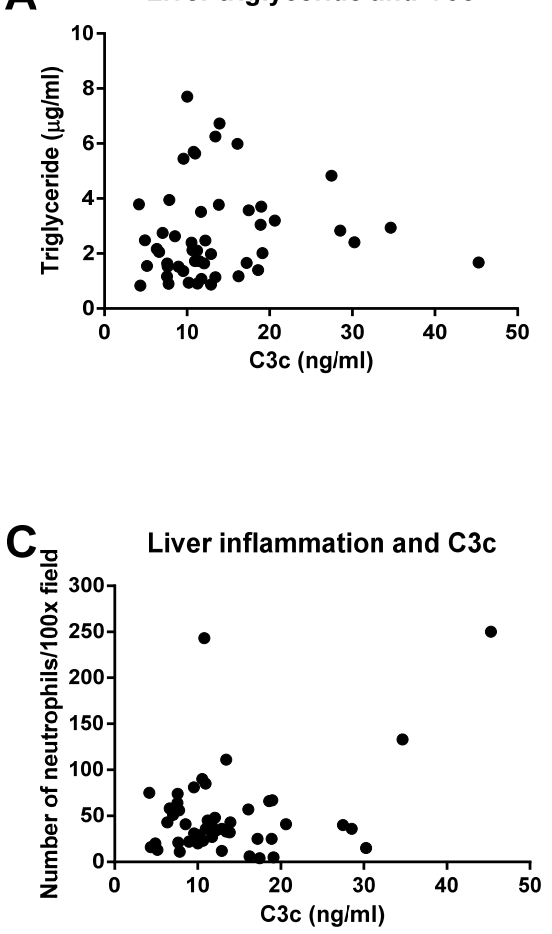

B
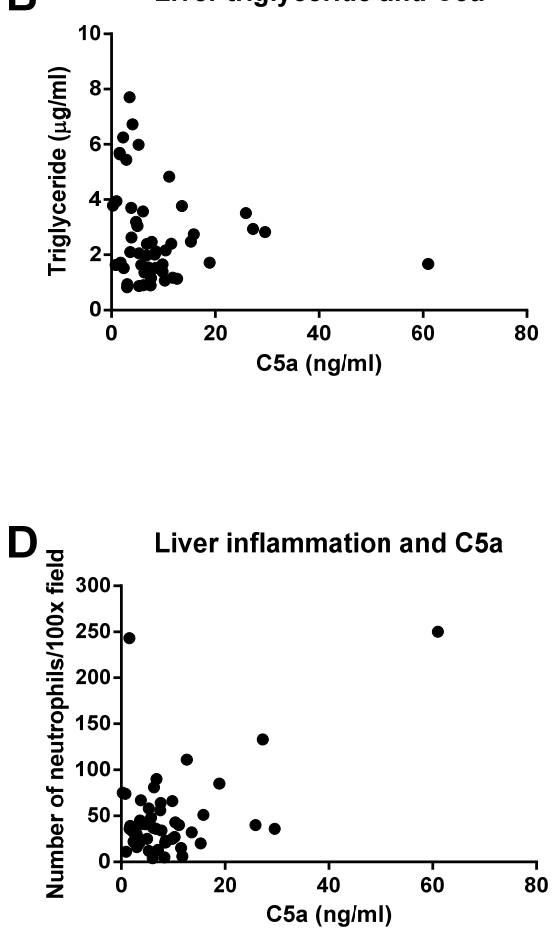

Figure 7.3 Hepatic concentrations of $\mathrm{C} 3 \mathrm{c}$ and $\mathrm{C} 5 \mathrm{a}$ are correlated with liver inflammation but not with steatosis.

A and B: Hepatic triglyceride concentration was not correlated with liver C3c (A) and C5a (B) levels, $r=0.070, p=0.627 ; r=-0.173, p=0.229$, respectively. C: Significant correlation between hepatic C3c concentrations and neutrophil infiltration, $r=0.373, p=0.008$. D: Highly significant correlation between hepatic C5a concentration and neutrophil infiltration, $r=0.485, p=0.0004$. 


\section{Discussion}

This study has shown that complement activation occurs in the non-tumour liver of patients with CRLM. Hepatic C5 activation was specifically increased in patients with nodular transformation and tended to be increased in patients with sinusoidal dilatation. Hepatic inflammation as reflected by neutrophil infiltration was markedly higher in patients with hepatic C3 and C5 activation. Steatosis was neither associated with activation of $\mathrm{C} 3$ nor with activation of $\mathrm{C} 5$.

The link between hepatic inflammation and complement activation has previously been shown in other liver diseases, including nonalcoholic and alcoholic fatty liver disease, viral hepatitis, and hepatic ischemia/reperfusion., ${ }^{8,21,23,24}$ It is likely related to the generation of $\mathrm{C} 3 \mathrm{a}$ and $\mathrm{C} 5 \mathrm{a}$, small complement peptides that have a strong chemotactic activity towards neutrophils and other leukocytes. ${ }^{25}$ These complement peptides are formed when C3 and C5 are activated through cleavage by C3 and C5 convertases. Activation of these complement factors may be initiated through the so-called classical pathway, the lectin pathway, and/or the alternative pathway. ${ }^{26}$ In the context of CALI, the induction of apoptosis in hepatocytes ${ }^{27}$ may drive complement activation through the classical pathway ${ }^{28}$. Apoptotic bodies bind to the complement component $\mathrm{C} 1 \mathrm{q}$ which assists in their clearance after subsequent activation of $\mathrm{C} 3$ and binding of C3b. C3b is then further metabolized generating $\mathrm{C} 3 \mathrm{c}$, one of the complement factors that we found to be detectable in the non-tumour liver of patients with CRLM.

It has previously been suggested that the alternative pathway of complement might be involved in sinusoidal obstruction syndrome. ${ }^{29}$ Patients with sinusoidal obstruction syndrome secondary to receiving a hematopoietic stem cell transplantation and cyclophosphamide treatment for acute myeloid leukaemia were found to have single nucleotide polymorphisms in the gene encoding the complement regulatory protein factor $\mathrm{H}^{29}$ These polymorphisms had previously been associated with atypical haemolytic uremic syndrome, a complement-driven disease. ${ }^{30}$ Complement activation was not significantly increased in subjects with sinusoidal dilatation in the present study, although we did observe a trend towards higher hepatic C5a levels in these patients. This could be related to the fact that the causes of sinusoidal dilatation in hematopoietic stem cell transplantation are different from those in sinusoidal dilatation. Hence, mechanisms underlying this type of liver injury are likely to be different as well.

Complement factors have also been shown to be involved in liver regeneration. In particular, C5 appears to be important given the severely compromised regenerative 
response after acute toxic liver injury in C5-deficient mice and the abrogation of hepatocyte proliferation after blockage of the $\mathrm{C} 5$ a receptor. ${ }^{16}$ By stimulating the $\mathrm{C} 5 \mathrm{a}$ receptor on the surface of Kupffer cells, C5a can modulate the release of IL- 6 and TNF- $\alpha$, which play a role in the initiation of hepatocyte proliferation. ${ }^{17}$ This important role of C5a in liver regeneration is supported by our data showing that C5a was elevated in patients with nodular transformation, which is considered to be a regenerative response to sinusoidal injury. ${ }^{20}$

It remains to be determined why TCC was not detectable in the liver even though $\mathrm{C} 3$ and C5 activation did occur. Formation of TCC is the ultimate result of complement activation, and TCC has a direct lytic capacity to kill injured cells. ${ }^{26}$ Hence, TCC activation is firmly regulated to avoid accidental cell damage. The lytic part of TCC can be removed within 5-10 minutes after deposition on the membrane of target cells through in-/outward vesiculation. ${ }^{31}$ In addition, the TCC component C8 can act as a TCC inhibitor. ${ }^{32}$ Furthermore, CD59, expressed on hepatocytes, negatively regulates TCC via interaction with the other TCC subunits, C5b-C9. ${ }^{12,26}$ Taken together, both the short half-life of TCC and its tight negative regulation might be accountable for the lack of detectable hepatic TCC in our tissue samples.

As a final remark, it should be mentioned that we analysed a relatively small number of patients. Therefore, the link between complement activation and hepatic nodularity needs to be validated by future research. Nevertheless, our data do provide the first evidence that various aspects of CALI are associated with complement activation. In view of this, investigation of the potential impact of complement inhibition using existing drugs $^{33-35}$ on the development of CALI is warranted. 


\section{References}

1. Kalady MF, de Campos-Lobato LF, Stocchi L, Geisler DP, Dietz D, Lavery IC, et al. Predictive factors of pathologic complete response after neoadjuvant chemoradiation for rectal cancer. Ann Surg 2009; 250(4):582-9.

2. Folprecht G, Gruenberger T, Bechstein WO, Raab HR, Lordick F, Hartmann JT, et al. Tumour response and secondary resectability of colorectal liver metastases following neoadjuvant chemotherapy with cetuximab: the CELIM randomised phase 2 trial. Lancet Oncol 2010; 11(1):38-47.

3. Rubbia-Brandt L, Lauwers GY, Wang H, Majno PE, Tanabe K, Zhu AX, et al. Sinusoidal obstruction syndrome and nodular regenerative hyperplasia are frequent oxaliplatin-associated liver lesions and partially prevented by bevacizumab in patients with hepatic colorectal metastasis. Histopathology 2010; 56(4):430-9.

4. Hubert C, Sempoux C, Horsmans Y, Rahier J, Humblet $Y$, Machiels JP, et al. Nodular regenerative hyperplasia: a deleterious consequence of chemotherapy for colorectal liver metastases? Liver Int 2007; 27(7):938-43.

5. Vigano L, Rubbia-Brandt L, De Rosa G, Majno P, Langella S, Toso C, et al. Nodular Regenerative Hyperplasia in Patients Undergoing Liver Resection for Colorectal Metastases After Chemotherapy: Risk Factors, Preoperative Assessment and Clinical Impact. Ann Surg Oncol 2015; 22(13):4149-57.

6. Robinson SM, Wilson $\mathrm{CH}$, Burt AD, Manas DM, White SA. Chemotherapy-associated liver injury in patients with colorectal liver metastases: a systematic review and meta-analysis. Ann Surg Oncol 2012; 19(13):4287-99.

7. Rubbia-Brandt L, Tauzin S, Brezault C, Delucinge-Vivier C, Descombes P, Dousset B, et al. Gene expression profiling provides insights into pathways of oxaliplatin-related sinusoidal obstruction syndrome in humans. Mol Cancer Ther 2011; 10(4):687-96.

8. Bajic G, Degn SE, Thiel S, Andersen GR. Complement activation, regulation, and molecular basis for complement-related diseases. EMBO J 2015; 34(22):2735-57.

9. Rensen SS, Slaats Y, Driessen A, Peutz-Kootstra CJ, Nijhuis J, Steffensen R, et al. Activation of the complement system in human nonalcoholic fatty liver disease. Hepatology 2009; 50(6):1809-17.

10. Yesilova Z, Ozata M, Oktenli C, Bagci S, Ozcan A, Sanisoglu SY, et al. Increased acylation stimulating protein concentrations in nonalcoholic fatty liver disease are associated with insulin resistance. Am J Gastroenterol 2005; 100(4):842-9.

11. Cianflone K, Vu H, Walsh M, Baldo A, Sniderman A. Metabolic response of Acylation Stimulating Protein to an oral fat load. J Lipid Res 1989; 30(11):1727-33.

12. Ricklin D, Hajishengallis G, Yang K, Lambris JD. Complement: a key system for immune surveillance and homeostasis. Nat Immunol 2010; 11(9):785-97.

13. Xu R, Lin F, He J, Jin L, Zhang JY, Fu J, et al. Complement 5 a stimulates hepatic stellate cells in vitro, and is increased in the plasma of patients with chronic hepatitis B. Immunology 2013; 138(3):228-34.

14. Schlaf G, Schmitz M, Heine I, Demberg T, Schieferdecker HL, Gotze O. Upregulation of fibronectin but not of entactin, collagen IV and smooth muscle actin by anaphylatoxin C5a in rat hepatic stellate cells. Histol Histopathol 2004; 19(4):1165-74.

15. Hillebrandt S, Wasmuth HE, Weiskirchen R, Hellerbrand C, Keppeler H, Werth A, et al. Complement factor 5 is a quantitative trait gene that modifies liver fibrogenesis in mice and humans. Nat Genet 2005; 37(8):835-43.

16. Mastellos D, Papadimitriou JC, Franchini S, Tsonis PA, Lambris JD. A novel role of complement: mice deficient in the fifth component of complement (C5) exhibit impaired liver regeneration. J Immuno/ 2001; 166(4):2479-86.

17. Strey CW, Markiewski M, Mastellos D, Tudoran R, Spruce LA, Greenbaum LE, et al. The proinflammatory mediators C3a and C5a are essential for liver regeneration. J Exp Med 2003; 198(6):913-23.

18. van den Broek MA, Shiri-Sverdlov R, Schreurs JJ, Bloemen JG, Bieghs V, Rensen SS, et al. Liver manipulation during liver surgery in humans is associated with hepatocellular damage and hepatic inflammation. Liver Int 2013; 33(4):633-41.

19. Vreuls CP, Van Den Broek MA, Winstanley A, Koek GH, Wisse E, Dejong CH, et al. Hepatic sinusoidal obstruction syndrome (SOS) reduces the effect of oxaliplatin in colorectal liver metastases.

Histopathology 2012; 61(2):314-8. 
20. Wanless IR. Micronodular transformation (nodular regenerative hyperplasia) of the liver: a report of 64 cases among 2,500 autopsies and a new classification of benign hepatocellular nodules. Hepatology 1990; 11(5):787-97.

21. Rensen SS, Slaats Y, Nijhuis J, Jans A, Bieghs V, Driessen A, et al. Increased hepatic myeloperoxidase activity in obese subjects with nonalcoholic steatohepatitis. Am J Pathol 2009; 175(4):1473-82.

22. Frey A, Ertl G, Angermann CE, Hofmann U, Stork S, Frantz S. Complement C3c as a biomarker in heart failure. Mediators Inflamm 2013; 2013:716902.

23. Jaeschke H, Hasegawa T. Role of neutrophils in acute inflammatory liver injury. Liver Int 2006; 26(8):9129.

24. Diepenhorst GM, de Graaf W, Niessen HW, van Vliet AK, Hack CE, van Gulik TM. Immunoglobulin M, Creactive protein and complement activation in rat hepatic ischemia-reperfusion injury. Eur Surg Res 2014; 52(1-2):50-62.

25. Klos A, Tenner AJ, Johswich KO, Ager RR, Reis ES, Kohl J. The role of the anaphylatoxins in health and disease. Mol Immunol 2009; 46(14):2753-66.

26. Merle NS, Church SE, Fremeaux-Bacchi V, Roumenina LT. Complement System Part I - Molecular Mechanisms of Activation and Regulation. Front Immunol 2015; 6:262.

27. Domart MC, Esposti DD, Sebagh M, Olaya N, Harper F, Pierron G, et al. Concurrent induction of necrosis, apoptosis, and autophagy in ischemic preconditioned human livers formerly treated by chemotherapy. $J$ Hepatol 2009; 51(5):881-9.

28. Nauta AJ, Castellano G, Xu W, Woltman AM, Borrias MC, Daha MR, et al. Opsonization with C1q and mannose-binding lectin targets apoptotic cells to dendritic cells. J Immunol 2004; 173(5):3044-50.

29. Bucalossi A, Toraldo F, Tozzi M, Lenoci M, Castagnini C, Artuso R, et al. Is complement alternative pathway disregulation involved in veno-occlusive disease of the liver? Biol Blood Marrow Transplant 2010; 16(12):1749-50.

30. Bernabeu-Herrero ME, Jimenez-Alcazar M, Anter J, Pinto S, Sanchez Chinchilla D, Garrido S, et al. Complement factor H, FHR-3 and FHR-1 variants associate in an extended haplotype conferring increased risk of atypical hemolytic uremic syndrome. Mol Immunol 2015; 67(2 Pt B):276-86.

31. Moskovich O, Fishelson Z. Live cell imaging of outward and inward vesiculation induced by the complement c5b-9 complex. J Biol Chem 2007; 282(41):29977-86.

32. Nemerow GR, Yamamoto KI, Lint TF. Restriction of complement-mediated membrane damage by the eighth component of complement: a dual role for C8 in the complement attack sequence. J Immunol 1979; 123(3):1245-52.

33. Hyzewicz J, Tanihata J, Kuraoka M, Nitahara-Kasahara Y, Beylier T, Ruegg UT, et al. Low-Intensity Training and the C5a Complement Antagonist NOX-D21 Rescue the mdx Phenotype through Modulation of Inflammation. Am J Pathol 2017; Doi. 10.1016/j.ajpath.2016.12.019.

34. Marshall KM, He S, Zhong Z, Atkinson C, Tomlinson S. Dissecting the complement pathway in hepatic injury and regeneration with a novel protective strategy. J Exp Med 2014; 211(9):1793-805.

35. Blom AM. The role of complement inhibitors beyond controlling inflammation. J Intern Med 2017; Doi. 10.1111/joim.12606. 


\section{Chapter}

Summary, implications, and future perspective 
A large body of literature has been published demonstrating the benefits of partial hepatectomy for increasing long-term survival in patients with colorectal liver metastases (CRLM). ${ }^{1}$ Surgery remains the mainstay of the CRLM treatment algorithm and provides the best prospect of cure. ${ }^{2,3}$ To further increase the cure rate, surgeons have developed many advanced surgical procedures for tumours traditionally considered irresectable, particularly multiple tumours with bilobular distribution. These procedures include two-stage hepatectomy, ${ }^{4}$ ultrasound-guided enhanced one-stage hepatectomy, ${ }^{5}$ and associating liver partition and portal vein ligation for staged hepatectomy (ALPPS). ${ }^{6,7}$ Apart from these advanced surgical techniques, neoadjuvant chemotherapy has been put forward as a treatment option that could reduce the tumour burden, thereby enabling surgical resection. ${ }^{1,8,9}$

Surgical safety, expressed as incidence of postoperative morbidity and mortality, is of great importance in the context of partial hepatectomy, and has been reported to be negatively influenced by neoadjuvant chemotherapy. ${ }^{10}$ In particular, the presence of chemotherapy-associated liver injury (CALI) has been shown to impair liver regeneration, which is pivotal for recovery of liver function in patients after partial hepatectomy. ${ }^{11}$ Moreover, CALI appears to be associated with reduced tumour responsiveness to neoadjuvant chemotherapy, ${ }^{12,13}$ which has implications for long-term recurrence-free survival and overall survival. Understanding the relationship between the impact of CALI on postoperative short- and long-term outcome, and the mechanisms underlying CALI is of key importance for improving surgical outcomes of patients undergoing partial hepatectomy for CRLM.

The central aim of the current thesis was to investigate the influence of CALI on postoperative short- and long-term outcome after partial hepatectomy for CRLM. This was then translated into three main specific aims: 1) to study the impact of CALI on short-term morbidity and mortality after partial hepatectomy for CRLM (Chapter 2, Chapter 3, and Chapter 4); 2) to investigate the influence of CALI on long-term recurrence-free survival and overall survival after partial hepatectomy for CRLM (Chapter 5); and 3) to provide better understanding of the mechanisms underlying CALI, the first step towards identification of potential biomarkers to predict CALI and of molecular targets to treat or prevent CALI (Chapter 6 and Chapter 7). 


\section{Part I. Influence of CALI on short-term outcome after partial hepatectomy for CRLM}

The first aim of this thesis was to assess the impact of CALI on short-term and long-term outcome after partial hepatectomy for CRLM. Chapter 2 describes a systematic review with meta-analysis conducted and reported in compliance with the relevant PRISMA and MOOSE guidelines on this important topic. ${ }^{14-16}$ We focused on the influence of chemotherapy-associated sinusoidal dilatation on morbidity after partial hepatectomy in patients with CRLM, and found that sinusoidal dilatation was not associated with any type of short-term outcome (i.e. overall morbidity, liver failure, liver-related morbidity, and mortality) after partial hepatectomy. It should be emphasized that the individual studies included in our meta-analysis had a considerable risk of bias ( with consideration of study participation, study attrition, prognostic factor measurement, outcome measurement, study confounding, and statistical analysis and reporting ${ }^{17}$ ) and comparatively low quality of outcome-specific evidence, leading to limited confidence in the overall conclusion that sinusoidal dilatation is not important for morbidity after partial hepatectomy for CRLM. Variations in study design and inclusion criteria, diverse definitions of a single endpoint, and varied endpoints under the category of morbidity were factors that negatively affected the quality of the evidence. In light of these concerns, no solid conclusions could be drawn and this study therefore cannot provide clinical advice on the topic.

\section{Implications of Chapter 2}

In the current era of evidence-based practice, meta-analyses or randomized controlled trials deliver the highest level of evidence. Conclusions drawn from these types of studies are therefore regularly incorporated into clinical guidelines. However, the definition, objectivity, and reproducibility of endpoints collected from studies performed in multiple centres determine whether multiple-origin data can be integrated and whether a robust conclusion can be drawn from a meta-analysis. Surgical outcome after partial hepatectomy must be standardized and defined in a way that enables clinicians to collect the data objectively and reproducibly. This will facilitate comparisons among multiple institutions, between different interventions, and within a single centre over time. ${ }^{18}$ Chapter 2 emphasizes the critical importance of assessing the risk of bias and the evaluation of quality in meta-analyses, to provide the most robust level of evidence for clinical decision making. More importantly, it highlights the need for unambiguous definitions of outcome in surgical oncology. 
In Chapter 3, the impact of chemotherapy-associated sinusoidal dilatation and additional major chemotherapy-associated non-tumour liver parenchymal damages (i.e. steatosis and steatohepatitis) on postoperative morbidity and mortality rates after partial hepatectomy was studied by performing a meta-analysis of individual participant data based on a systematic literature review. A comprehensive literature search was first performed following PRISMA and Moose guidelines to identify studies relevant to the aim. ${ }^{14,15}$ Corresponding authors from each study that fulfilled the inclusion criteria were contacted subsequently for collaboration and for sharing original individual participant data of the published cohort. Next, aggregated data from each collaborator was reconstructed, pooled, and modelled simultaneously. A consolidate database comprising 788 patients who underwent partial hepatectomy for CRLM in eight centres was generated. The influence of preoperative chemotherapeutic agents on liver injury, and subsequently the effect of liver injury on short-term outcome after liver surgery were analysed applying one-step uni-/multi- variable binary logistic regression models.

We observed an increase in postoperative major morbidity (Dindo-Clavien grade III-V ${ }^{19}$ ) and liver surgery-specific complications (i.e. ascites, postoperative liver failure, bile leakage, intra-abdominal abscess, intra-abdominal haemorrhage, and operative mortality ${ }^{20}$ ) after partial hepatectomy in patients with severe sinusoidal dilatation (grade 2-3) and steatohepatitis (non-alcoholic steatohepatitis score, NAS $\geq 4$ ), respectively. Moreover, postoperative liver failure occurred more often in patients with severe sinusoidal dilatation. With respect to steatohepatitis, lobular inflammation, but not steatosis or hepatocellular ballooning, was strongly linked to increased postoperative morbidity. Oxaliplatin was a strong risk factor for severe sinusoidal dilatation in multivariable analysis, whereas the addition of bevacizumab was related to a twofold decrease in the occurrence of severe sinusoidal dilatation in patients who received oxaliplatin. We concluded that after partial hepatectomy, severe sinusoidal dilatation and steatohepatitis were related to increased postoperative major morbidity and liver surgery-specific complications. Moreover, postoperative liver failure occurred more often in patients with severe sinusoidal dilatation. Oxaliplatin was a strong risk factor for the development of severe sinusoidal dilatation, whereas the addition of bevacizumab to oxaliplatin-based regimen reduced the occurrence of severe sinusoidal dilatation.

\section{Implications of Chapter 3}

Individual participant data analysis offers many advantages. In particular, it reduces reliance on the reporting quality of individual studies. This allowed us to re-define outcome by applying a unified definition. Furthermore, we were now able to choose a consistent analysis approach. This resulted in the first study to demonstrate both the 
effect of chemotherapy on liver injury and, subsequently, the effect of liver injury on short-term postoperative outcome in a large, multicentre patient cohort. In view of the developed measures to diagnose CALI preoperatively (e.g. by imaging modalities, histologic assessment, and biochemical analyses), ${ }^{21}$ and the negative relationship between CALI and postoperative morbidity, it is advised to adapt surgical management when CALI is confirmed, for instance by applying ablative therapy or two-stage hepatectomy. Moreover, with increased chemotherapy-related postoperative morbidity, decreased chemotherapy responsiveness, ${ }^{12}, 13$ and increasing doubts about the usefulness of neoadjuvant chemotherapy in certain patient groups, ${ }^{22}$ one could even speculate that some patients would benefit from immediate resection instead of neoadjuvant chemotherapeutical treatment.

Chapter 4 describes the impact of CALI on infectious complications after partial hepatectomy for CRLM. This topic could not be addressed in the systematic reviews described in chapters 2 and 3 since there are hardly any studies conducted on this topic. This chapter describes a study of 265 consecutive patients who had undergone partial hepatectomy for CRLM at Maastricht University Medical Centre. Data regarding postoperative infectious complications were prospectively monitored daily by an independent infection-control nurse. CALI, as reflected by sinusoidal dilatation, steatosis, and steatohepatitis was assessed by three experienced liver pathologists. We also monitored nodular regeneration, another type of liver injury that is known to be tightly associated with oxaliplatin-based treatment. ${ }^{23}$ It is recently gaining attention as postoperative liver failure appears to occur more often when its presence is observed. ${ }^{23}$ We observed that postoperative surgical site infections and systemic sepsis after partial hepatectomy for CRLM occurred more often in patients with hepatic steatosis, whereas neither sinusoidal dilatation nor nodular regeneration was related to infectious complications. Presence of severe surgical site infections (i.e. deep surgical site infections and intra-abdominal abscesses) was associated with increased 90-day mortality, prolonged length of hospital stay, decreased long-term recurrence-free survival, and reduced overall survival. In contrast, none of the studied expressions of CALI was related to survival. Whereas there was an inverse relation between severe steatosis and sinusoidal dilatation, nodular regeneration was positively correlated to the occurrence of sinusoidal dilatation. Irinotecan-based treatment and body mass index were associated with steatosis. We concluded that chemotherapy-associated steatosis was associated with increased infectious complications. The latter, in turn, was related to decreased recurrence-free survival and overall survival. 


\section{Implications of Chapter 4}

Despite mechanisms underlying the negative relationship between postoperative surgical site infections and long-term survival remain unclear, overall body immunocompetence may play a crucial role, as supported by the following observations: surgical infections occur more often once the body immune system is suppressed. ${ }^{24}$ Efforts to enhance the immune system may suppress tumour progression, ${ }^{25}$ increase tumour response, and prolong long-term survival. ${ }^{26}$ In essence, the presence of surgical site infections may reflect a relatively weaker innate or adaptive immunity. Tumour suppression may therefore be weakened, eventually resulting in more frequent tumour recurrence and decreased long-term overall survival.

Tumour response, which is expressed as tumour regression grade and represents the effect of chemotherapy on the tumour, has been shown to be positively associated with survival, ${ }^{13}$ whereas negatively related to the presence of chemotherapy-associated liver injury. ${ }^{12}$ However, the influence of tumour regression grade on survival was not taken into account in this study. Therefore, future study is needed to clarify whether CALI itself or an impaired tumour response as a result of CALI is the key component associated with long-term recurrence-free survival and overall survival after partial hepatectomy for colorectal liver metastases. Given that postoperative infectious complications were found to be important prognostic factors for survival, such factors are advised to be included as confounders in future studies.

Steatohepatitis was previously determined by assessing the so-called nonalcoholic steatohepatitis activity score (NAS), which summarizes the severity of steatosis, lobular inflammation, and hepatocellular ballooning. ${ }^{27}$ For the purpose of reducing interobserver variations among pathologists, a novel scoring system (steatosis, activity, and fibrosis [SAF]) has been proposed in 2014, ${ }^{28}$ and was applied to define steatohepatitis in this chapter. Surprisingly, application of the novel SAF scoring system was related to a very low incidence of steatohepatitis $(0.4 \%)$ in our cohort. Although this low incidence could also be due to the fact that a minority of patients in the present cohort was treated with irinotecan, a known risk factor for steatohepatitis, ${ }^{29-31}$ future studies assessing steatohepatitis by applying the SAF scoring system need to take this observed low incidence into account in the light of the number of patients to be included.

With respect to the adverse effect of hepatic steatosis on superficial surgical site infections and systemic sepsis, clinicians are advised to adopt measures for infection control when suspected hepatic steatosis is confirmed for instance through preoperative ultrasound or radiological images, or even seeing "yellow liver" during surgery. 
Moreover, given that postoperative infectious complications were substantial prognostic factors for long-term survival, fundamental research dedicated to mechanisms underlying this observation are of interest. In addition, it is advised for future studies to consider postoperative infectious complications as potential confounders when identifying predictors for survival.

Chapter 5 summarizes a study which aimed to 1) validate a potential negative influence of CALI on tumour responsiveness to therapy, and 2) clarify the impact of CALI on longterm survival after partial hepatectomy for CRLM. In this study, we included 166 consecutive patients who were treated with neoadjuvant chemotherapy and underwent subsequent partial hepatectomy for CRLM. Since infectious complications were shown to be significant predictors of shortened long-term survival in Chapter 4, we specifically included them as confounder. Of all studied background liver abnormalities, only sinusoidal dilatation was related to diminished complete tumour response after neoadjuvant chemotherapy. As expected, complete tumour response was strongly associated with prolonged recurrence-free survival and overall survival. None of the background liver parenchymal abnormalities was associated with survival. Addition of bevacizumab chemotherapy was shown to decrease the incidence of sinusoidal dilatation and tended to be related to increased complete tumour response. We concluded that CALI was not related to survival. CALI was, however, associated with diminished complete tumour response, and tumour response, in turn, was associated with survival.

\section{Implications of Chapter 5}

Although sinusoidal dilatation was associated with diminished complete tumour response, and tumour response was associated with long-term survival, sinusoidal dilatation itself was not correlated with survival. Because neoadjuvant chemotherapy provides an increased complete tumour response ${ }^{32}$ which is related to prolonged survival, it could be speculated that the benefits of neoadjuvant chemotherapy in terms of survival outweigh the harms of inducing undesired parenchymal liver injury. In other words, chemotherapy could be more beneficial if clinicians could reduce the negative effects of CALI. Since addition of bevacizumab to neoadjuvant chemotherapy was associated with reduced sinusoidal dilatation and tended to be related to increased tumour response, it seems beneficial to add bevacizumab to oxaliplatin-based neoadjuvant chemotherapy. 


\section{Part II. Mechanisms underlying CALI}

Previous chapters have demonstrated that CALI frequently occurred in patients who received chemotherapy, and that it was related to increased postoperative morbidity, and even compromised tumour responsiveness to neoadjuvant chemotherapy. Our next aim was therefore to provide a better understanding of the mechanisms underlying CALI, the first step towards identification of biomarkers for its preoperative diagnosis as well as therapeutic targets.

Chapter 6 summarizes a fundamental study which investigated the non-tumour hepatic microRNA expression profile in a setting of oxaliplatin-associated sinusoidal dilatation. In this study, we included a total of 28 patients who were treated with preoperative oxaliplatin-based chemotherapy and subsequently underwent partial hepatectomy for CRLM at Maastricht University Medical Centre. Ten patients with moderate to severe sinusoidal dilatation were matched with ten patients without sinusoidal dilatation by parameters which we suspected to affect microRNA expression (i.e. number of oxaliplatin cycles, cumulative oxaliplatin dose, sex, age, body mass index, and liver transaminases). MicroRNA expression was analyzed in the matched cohort through Agilent microarrays. Because the data were analysed without false discovery rate correction and there was potential bias caused by relatively small cohort size, a validation by qPCR was subsequently performed to confirm the findings from the microRNA array. We found that sinusoidal dilatation was not associated with upregulation of any microRNA, but was related to downregulation of miR-21 and miR150. These findings were confirmed in a slightly larger Maastricht cohort of 28 patients. However, the downregulation of miR-21 and miR-150 in the liver of patients with sinusoidal dilatation was not found in plasma of the same patients. Given that miR-21 and miR-150 play important roles in tumour progression, affecting survival in colorectal cancer patients, ${ }^{33,34}$ and since sinusoidal dilatation compromises tumour response ${ }^{12,13}$ and may be further negatively related to survival, ${ }^{35}$ we next explored the potential link between sinusoidal dilatation, miR-21 and miR-150 expression, tumour response, and survival. We found that sinusoidal dilatation was not associated with survival. However, low expression of miR-21 and miR-150 was related to impaired tumour response, and shortened recurrence-free as well as overall survival. We concluded that sinusoidal dilatation was not related to survival. Sinusoidal dilatation was, however, associated with expression levels of hepatic miR-21 and miR-150, and miR-21 and miR-150, in turn, were related to survival. 


\section{Implication of Chapter 6}

Oxaliplatin-induced liver toxicity initially depletes glutathione and causes F-actin depolymerization, which results in rounding up and subsequent dehiscence of sinusoidal endothelial cells and obstruction of sinusoidal blood flow leading to sinusoidal dilatation. ${ }^{36-39}$ In line with these observations, gene expression of vascular endothelial growth factor (VEGF), a factor that is known to induce endothelial cell migration and mediate angiogenesis, is upregulated in oxaliplatin-related sinusoidal dilatation, ${ }^{31,40}$ supporting that sinusoidal endothelial cells play a crucial role at the early stage of sinusoidal dilatation. Given that VEGF is targeted by miR-150, ${ }^{41}$ sinusoidal dilatationassociated VEGF upregulation is consistent with the downregulation of miR-150 that we observed in the liver of patients with sinusoidal dilatation, and suggests that low miR150 levels may contribute to the observed increase in VEGF. Similar to miR-150, miR-21 overexpression has been shown to suppress endothelial cell proliferation and migration of endothelial cells by inhibiting RhoB. ${ }^{42}$ In view of the important role that sinusoidal endothelial cells are playing in the development of sinusoidal dilatation, and the tight relationship between endothelial cells and miR-21/miR-150, our findings provide novel insight into the mechanisms of oxaliplatin-induced sinusoidal dilatation. Furthermore, given that microRNAs could be potential therapeutic options ${ }^{43}$ to treat disease and treatment for sinusoidal dilatation is lacking, it is worth investigating that whether the occurrence of sinusoidal dilatation could be reduced by providing miR-21 and miR-150 as gene therapy.

The relationship between sinusoidal dilatation-associated microRNAs and survival is interesting. Although numerous studies reveal that miR-21 and miR-150 in tumour tissue play important roles in tumour proliferation and migration, ${ }^{33,44,41}$ which affects longterm survival, ${ }^{34,45}$ the impact of microRNAs in non-tumour liver parenchymal on the behavior of tumour is unknown. Given the suppressive action of miR-21 and miR-150 on endothelial cells, we could speculate that tumour angiogenesis occurring at the borderline area of normal liver and tumour may be inhibited, thereby contributing to increased long-term survival. However, in view of the cohort size, interpretation of findings concerning sinusoidal dilatation-related microRNAs in non-tumour liver and survival should be prudent, and further validation in a large cohort is encouraged and warranted. Because the downregulation of miR-21 and miR-150 in the liver of patients with sinusoidal dilatation was not found in plasma, these microRNAs are unlikely to be useful biomarkers of sinusoidal dilatation.

Chapter 7 describes the relationship between hepatic complement activation and the presence of CALI in patients with CRLM. 50 consecutive patients who underwent partial 
hepatectomy for CRLM were included. Sinusoidal dilatation and nodular regeneration were assessed using formalin-fixed non-tumour liver samples. Several analyses were performed using frozen samples collected before manipulating the intestines or liver, which has been shown to limit inflammation due to sample collection. ${ }^{46} \mathrm{C} 3 \mathrm{c}, \mathrm{C} 5 \mathrm{a}$, and terminal complement complex (TCC) concentrations in liver tissue lysates were determined by ELISA to investigate complement activation; neutrophil infiltration was assessed by myeloperoxidase staining and subsequently quantified as a measure of lobular inflammation; hepatic triglyceride concentration was analysed to quantify steatosis. We found that hepatic $\mathrm{C} 3 \mathrm{c}$ and $\mathrm{C5}$ a were both detectable, with $\mathrm{C3c}$ concentrations strongly correlating to $\mathrm{C} 5 \mathrm{a}$ concentrations, consistent with persistent complement activation. Hepatic C5 activation was specifically increased in patients with nodular transformation and tended to be increased in patients with sinusoidal dilatation. Hepatic inflammation as reflected by neutrophil infiltration was markedly higher in patients with hepatic C3 and C5 activation. Steatosis was neither associated with activation of $\mathrm{C} 3$ nor with activation of $\mathrm{C} 5$. We concluded that complement activation occurs in the non-tumour liver of patients with CRLM, and that it was associated with the presence of hepatic nodular transformation and lobular inflammation.

\section{Implications of Chapter 7}

The thought of the complement system being involved in the development of steatosis and steatohepatitis was due to the fact that acylation stimulating protein, which is generated from $\mathrm{C} 3 \mathrm{a}$, stimulates triglyceride synthesis. ${ }^{47}$ However, our data demonstrated that complement activation was only related to increased lobular inflammation but not hepatic triglyceride concentration, implying that complement activation only plays a role in hepatitis and the development of steatosis is probably not driven by mechanisms related to acylation stimulating protein. Our findings were supported by previous studies which show increased acylation stimulating protein ${ }^{48}$ and activated complement system ${ }^{49,50}$ in steatohepatitis but not steatosis.

Although the cross-sectional design of the study precludes drawing conclusions on a causal link between complement activation and CALI, our data do provide evidence that various aspects of CALI are associated with complement activation. In view of the tight relationship between CALI and complement activation, and that diseases driven by complement activation could be treated with complement inhibitors, ${ }^{49-51}$ it would be of interest to study the impact of complement inhibition on the prevention of CALI using existing drugs. 


\section{References}

1. Alberts SR. Evolving role of chemotherapy in resected liver metastases. J Clin Oncol 2006; 24(31):4952-3.

2. Kanas GP, Taylor A, Primrose JN, Langeberg WJ, Kelsh MA, Mowat FS, et al. Survival after liver resection in metastatic colorectal cancer: review and meta-analysis of prognostic factors. Clin Epidemiol 2012; 4:283301.

3. Scheele J. Hepatectomy for liver metastases. Br J Surg 1993; 80(3):274-6.

4. Adam R, Laurent A, Azoulay D, Castaing D, Bismuth H. Two-stage hepatectomy: A planned strategy to treat irresectable liver tumors. Ann Surg 2000; 232(6):777-85.

5. Torzilli G, Procopio F, Botea F, Marconi M, Del Fabbro D, Donadon M, et al. One-stage ultrasonographically guided hepatectomy for multiple bilobar colorectal metastases: a feasible and effective alternative to the 2-stage approach. Surgery 2009; 146(1):60-71.

6. Schnitzbauer AA, Lang SA, Goessmann H, Nadalin S, Baumgart J, Farkas SA, et al. Right portal vein ligation combined with in situ splitting induces rapid left lateral liver lobe hypertrophy enabling 2-staged extended right hepatic resection in small-for-size settings. Ann Surg 2012; 255(3):405-14.

7. de Santibanes E, Clavien PA. Playing Play-Doh to prevent postoperative liver failure: the "ALPPS" approach. Ann Surg 2012; 255(3):415-7.

8. Adam R, Delvart V, Pascal G, Valeanu A, Castaing D, Azoulay D, et al. Rescue surgery for unresectable colorectal liver metastases downstaged by chemotherapy: a model to predict long-term survival. Ann Surg 2004; 240(4):644-57; discussion 657-8.

9. Lam VW, Spiro C, Laurence JM, Johnston E, Hollands MJ, Pleass HC, et al. A systematic review of clinical response and survival outcomes of downsizing systemic chemotherapy and rescue liver surgery in patients with initially unresectable colorectal liver metastases. Ann Surg Oncol 2012; 19(4):1292-301.

10. Klevebro F, Lindblad M, Johansson J, Lundell L, Nilsson M. Outcome of neoadjuvant therapies for cancer of the oesophagus or gastro-oesophageal junction based on a national data registry. Br J Surg 2016; 103(13):1864-1873.

11. Narita M, Oussoultzoglou E, Chenard MP, Rosso E, Casnedi S, Pessaux P, et al. Sinusoidal obstruction syndrome compromises liver regeneration in patients undergoing two-stage hepatectomy with portal vein embolization. Surg Today 2011; 41(1):7-17.

12. Vreuls CP, Van Den Broek MA, Winstanley A, Koek GH, Wisse E, Dejong CH, et al. Hepatic sinusoidal obstruction syndrome (SOS) reduces the effect of oxaliplatin in colorectal liver metastases. Histopathology 2012; 61(2):314-8.

13. Vigano L, Capussotti L, De Rosa G, De Saussure WO, Mentha G, Rubbia-Brandt L. Liver resection for colorectal metastases after chemotherapy: impact of chemotherapy-related liver injuries, pathological tumor response, and micrometastases on long-term survival. Ann Surg 2013; 258(5):731-40; discussion $741-2$.

14. Moher D, Liberati A, Tetzlaff J, Altman DG, Group P. Preferred reporting items for systematic reviews and meta-analyses: the PRISMA statement. Ann Intern Med 2009; 151(4):264-9, W64.

15. Stroup DF, Berlin JA, Morton SC, Olkin I, Williamson GD, Rennie D, et al. Meta-analysis of observational studies in epidemiology: a proposal for reporting. Meta-analysis Of Observational Studies in Epidemiology (MOOSE) group. JAMA 2000; 283(15):2008-12.

16. Hayden JA, Chou R, Hogg-Johnson S, Bombardier C. Systematic reviews of low back pain prognosis had variable methods and results: guidance for future prognosis reviews. J Clin Epidemiol 2009; 62(8):781-796 e1.

17. Hayden JA, Cote P, Bombardier C. Evaluation of the quality of prognosis studies in systematic reviews. Ann Intern Med 2006; 144(6):427-37.

18. Clavien PA, Sanabria JR, Strasberg SM. Proposed classification of complications of surgery with examples of utility in cholecystectomy. Surgery 1992; 111(5):518-26.

19. Dindo D, Demartines N, Clavien PA. Classification of surgical complications: a new proposal with evaluation in a cohort of 6336 patients and results of a survey. Ann Surg 2004; 240(2):205-13.

20. van den Broek MA, van Dam RM, van Breukelen GJ, Bemelmans MH, Oussoultzoglou E, Pessaux P, et al. Development of a composite endpoint for randomized controlled trials in liver surgery. Br J Surg 2011; 98(8):1138-45. 
21. van Mierlo KM, Schaap FG, Dejong CH, Olde Damink SW. Liver resection for cancer: New developments in prediction, prevention and management of postresectional liver failure. J Hepatol 2016; 65(6):1217-1231.

22. Khoo E, O'Neill S, Brown E, Wigmore SJ, Harrison EM. Systematic review of systemic adjuvant, neoadjuvant and perioperative chemotherapy for resectable colorectal-liver metastases. HPB (Oxford) 2016; 18(6):485-93.

23. Vigano L, Rubbia-Brandt L, De Rosa G, Majno P, Langella S, Toso C, et al. Nodular Regenerative Hyperplasia in Patients Undergoing Liver Resection for Colorectal Metastases After Chemotherapy: Risk Factors, Preoperative Assessment and Clinical Impact. Ann Surg Oncol 2015; 22(13):4149-57.

24. Akhter MS, Verma R, Madhukar KP, Vaishampayan AR, Unadkat PC. Incidence of surgical site infection in postoperative patients at a tertiary care centre in India. J Wound Care 2016; 25(4):210-2, 214-7.

25. Shrivastava $P$, Singh SM, Singh N. Effect of thymosin alpha 1 on the antitumor activity of tumorassociated macrophage-derived dendritic cells. J Biomed Sci 2004; 11(5):623-30.

26. Lipson SD, Chretien PB, Makuch R, Kenady DE, Cohen MH. Thymosin immunotherapy in patients with small cell carcinoma of the lung: correlation of in vitro studies with clinical course. Cancer 1979; 43(3):863-70.

27. Kleiner DE, Brunt EM, Van Natta M, Behling C, Contos MJ, Cummings OW, et al. Design and validation of a histological scoring system for nonalcoholic fatty liver disease. Hepatology 2005; 41(6):1313-21.

28. Bedossa P, Consortium FP. Utility and appropriateness of the fatty liver inhibition of progression (FLIP) algorithm and steatosis, activity, and fibrosis (SAF) score in the evaluation of biopsies of nonalcoholic fatty liver disease. Hepatology 2014; 60(2):565-75.

29. Morris-Stiff G, Tan YM, Vauthey JN. Hepatic complications following preoperative chemotherapy with oxaliplatin or irinotecan for hepatic colorectal metastases. Eur J Surg Oncol 2008; 34(6):609-14.

30. Khan AZ, Morris-Stiff G, Makuuchi M. Patterns of chemotherapy-induced hepatic injury and their implications for patients undergoing liver resection for colorectal liver metastases. J Hepatobiliary Pancreat Surg 2009; 16(2):137-44.

31. Robinson SM, Wilson $\mathrm{CH}$, Burt AD, Manas DM, White SA. Chemotherapy-associated liver injury in patients with colorectal liver metastases: a systematic review and meta-analysis. Ann Surg Oncol 2012; 19(13):4287-99.

32. Folprecht G, Gruenberger T, Bechstein WO, Raab HR, Lordick F, Hartmann JT, et al. Tumour response and secondary resectability of colorectal liver metastases following neoadjuvant chemotherapy with cetuximab: the CELIM randomised phase 2 trial. Lancet Oncol 2010; 11(1):38-47.

33. Feng J, Yang $Y$, Zhang $P$, Wang F, Ma Y, Qin H, et al. miR-150 functions as a tumour suppressor in human colorectal cancer by targeting c-Myb. J Cell Mol Med 2014; 18(10):2125-34.

34. Ma Y, Zhang P, Wang F, Zhang H, Yang J, Peng J, et al. miR-150 as a potential biomarker associated with prognosis and therapeutic outcome in colorectal cancer. Gut 2012; 61(10):1447-53.

35. Tamandl D, Klinger M, Eipeldauer S, Herberger B, Kaczirek K, Gruenberger B, et al. Sinusoidal obstruction syndrome impairs long-term outcome of colorectal liver metastases treated with resection after neoadjuvant chemotherapy. Ann Surg Oncol 2011; 18(2):421-30.

36. DeLeve LD, Ito Y, Bethea NW, McCuskey MK, Wang X, McCuskey RS. Embolization by sinusoidal lining cells obstructs the microcirculation in rat sinusoidal obstruction syndrome. Am J Physiol Gastrointest Liver Physiol 2003; 284(6):G1045-52.

37. Rubbia-Brandt L, Audard V, Sartoretti P, Roth AD, Brezault C, Le Charpentier M, et al. Severe hepatic sinusoidal obstruction associated with oxaliplatin-based chemotherapy in patients with metastatic colorectal cancer. Ann Oncol 2004; 15(3):460-6.

38. Vreuls CP, Driessen A, Olde Damink SW, Koek GH, Duimel H, van den Broek MA, et al. Sinusoidal obstruction syndrome (SOS): A light and electron microscopy study in human liver. Micron 2016; 84:1722.

39. Ezzat T, van den Broek MA, Davies N, Dejong $\mathrm{CH}$, Bast A, Malago $\mathrm{M}$, et al. The flavonoid monoHER prevents monocrotaline-induced hepatic sinusoidal injury in rats. J Surg Oncol 2012; 106(1):72-8.

40. Rubbia-Brandt L, Tauzin S, Brezault C, Delucinge-Vivier C, Descombes P, Dousset B, et al. Gene expression profiling provides insights into pathways of oxaliplatin-related sinusoidal obstruction syndrome in humans. Mol Cancer Ther 2011; 10(4):687-96.

41. Yu ZY, Bai YN, Luo LX, Wu H, Zeng Y. Expression of microRNA-150 targeting vascular endothelial growth factor-A is downregulated under hypoxia during liver regeneration. Mol Med Rep 2013; 8(1):287-93. 
42. Connolly EC, Van Doorslaer K, Rogler LE, Rogler CE. Overexpression of miR-21 promotes an in vitro metastatic phenotype by targeting the tumor suppressor RHOB. Mol Cancer Res 2010; 8(5):691-700.

43. Janssen HL, Reesink HW, Lawitz EJ, Zeuzem S, Rodriguez-Torres M, Patel K, et al. Treatment of HCV infection by targeting microRNA. N Engl J Med 2013; 368(18):1685-94.

44. Wang WH, Chen J, Zhao F, Zhang BR, Yu HS, Jin HY, et al. MiR-150-5p suppresses colorectal cancer cell migration and invasion through targeting MUC4. Asian Pac J Cancer Prev 2014; 15(15):6269-73.

45. Menendez P, Padilla D, Villarejo P, Palomino T, Nieto P, Menendez JM, et al. Prognostic implications of serum microRNA-21 in colorectal cancer. J Surg Oncol 2013; 108(6):369-73.

46. van den Broek MA, Shiri-Sverdlov R, Schreurs JJ, Bloemen JG, Bieghs V, Rensen SS, et al. Liver manipulation during liver surgery in humans is associated with hepatocellular damage and hepatic inflammation. Liver Int 2013; 33(4):633-41.

47. Cianflone K, Vu H, Walsh M, Baldo A, Sniderman A. Metabolic response of Acylation Stimulating Protein to an oral fat load. J Lipid Res 1989; 30(11):1727-33.

48. Yesilova Z, Ozata M, Oktenli C, Bagci S, Ozcan A, Sanisoglu SY, et al. Increased acylation stimulating protein concentrations in nonalcoholic fatty liver disease are associated with insulin resistance. $\mathrm{Am} \mathrm{J}$ Gastroenterol 2005; 100(4):842-9.

49. Hyzewicz J, Tanihata J, Kuraoka M, Nitahara-Kasahara Y, Beylier T, Ruegg UT, et al. Low-Intensity Training and the C5a Complement Antagonist NOX-D21 Rescue the mdx Phenotype through Modulation of Inflammation. Am J Pathol 2017.

50. Marshall KM, He S, Zhong Z, Atkinson C, Tomlinson S. Dissecting the complement pathway in hepatic injury and regeneration with a novel protective strategy. J Exp Med 2014; 211(9):1793-805.

51. Blom AM. The role of complement inhibitors beyond controlling inflammation. J Intern Med 2017. 
Valorisation 



\section{Valorisation}

To transfer academic research findings for the benefit of the society is one of the main tasks of Dutch universities by law. ${ }^{1}$ Therefore, it is obligated to translate academic wisdom to societal benefit in ways that go beyond numerous academic findings. This is especially the case when research has been sponsored by foundations, non-profit organizations, taxpayers, or charities. This chapter is intended to describe how the research results presented in this thesis could be used in clinical practice and subsequently how a broadest community could benefit.

\section{Relevance}

\section{Scientific relevance}

Annually, approximately half of globally 1.3 million patients with colorectal cancer develop colorectal liver metastases (CRLM), ${ }^{2-4}$ of whom over $75 \%$ are not eligible for curative hepatic resection. ${ }^{4,5}$ Neoadjuvant chemotherapy enhances resectability by approximately $15 \%$ in this group, ${ }^{6,7}$ but has been associated with regimen-specific parenchymal liver injury, collectively referred to as chemotherapy-associated liver injury (CALI, i.e. sinusoidal dilatation, nodular regeneration, steatosis, and steatohepatitis). ${ }^{8-11}$ Once occurred, CALI can persist for at least nine months even after cessation of chemotherapy. ${ }^{12}$ The potential downstream side-effects of CALI on clinical outcome (i.e. postoperative morbidity, mortality, and long-term survival) after partial hepatectomy are an active area of research surrounded with controversy, and the molecular mechanisms underlying its development are still mostly unknown. Therefore, the central theme of the studies conducted in the present thesis was to unravel these questions.

\section{Economic relevance}

The current thesis has revealed that CALI is a risk factor for increased postoperative morbidity after partial hepatectomy for colorectal liver metastases. The presence of increased morbidity results in a prolonged length of hospital stay and may therefore eventually increase hospital cost. Moreover, in many cases, after discharge from the hospital, patients with postoperative morbidity remain to have the need of being followed by the first referral doctors (e.g. general practitioner) for a certain period. Therefore, it is reasonable to speculate that the increased cost as a consequence of extra health-care may eventually burden the government finance. Based on the findings from the current thesis, we were able to advise clinicians to adapt surgical management once CALI is diagnosed preoperatively or observed during liver surgery (e.g., 'blue' or 
'yellow' liver). Therefore, the side-effects of CALI on postoperative morbidity and, as a consequence, the effects of CALI on the economic burden could be limited.

\section{Societal relevance}

Increased postoperative morbidity due to the presence of CALI has important potential negative influences on society. This is related to increased financial cost, but also to patients spending increased time on health-care related services, and, as a consequence, taking more sick leaves and spending less time on work. This negative influence may even radiate from the employer to the entire work-related network. Moreover, this thesis demonstrated that the presence of sinusoidal dilatation diminished tumour response to neoadjuvant chemotherapy. Of note, complete tumour response would allow patients to receive future curative surgical treatment and result in an increased tumour recurrence-free survival and overall survival. However, the diminished tumour response due to the occurrence of sinusoidal dilatation may alter the treatment strategy from curative to palliative. The latter, unfortunately, is related to a shorter lifespan. Additionally, being sick negatively affects quality of life, and the side effects of CALI are usually persistent for months. ${ }^{12}$ Importantly, addition of bevacizumab to oxaliplatinbased chemotherapeutic regimens has been confirmed to be a beneficial approach to prevent sinusoidal dilatation, thereafter minimizing its negative effect on tumour response. Moreover, the development of oxaliplatin-induced sinusoidal dilatation, which we suggested to be related to the low expression of hepatic miR-21 and miR-150, may be prevented by treatment targeting these microRNAs in the future. With these evidence-based advices, patients would benefit from personalized treatment by prevented sinusoidal dilatation, increased tumour response, increased lifetime without tumour recurrence, and even increased lifespan. As a consequence, the negative influence of CALI on the society could be diminished.

\section{Target groups}

CRLM treatment involves multidisciplinary clinicians (e.g. gastroenterologists, surgeons, oncologists, and radiologists). The entire studies performed in this thesis focused on the population of patients with this disease. Therefore, in addition to the academic community, the research results benefit the group of patients with primary colorectal cancer and those with or without liver metastases. Additionally, our findings may also benefit paediatric patients who undergo hematopoietic stem cell transplantation and develop busulfan-induced hepatic sinusoidal injury ${ }^{13,14}$; and those with breast cancer who develop tamoxifen-induced nonalcoholic steatohepatitis. ${ }^{15}$ 


\section{Innovation and Implementation}

The studies conducted in this thesis are novel and elaborated important clinical concerns. For instance, a first meta-analysis which aimed to study the downstream effect of CALI on postoperative morbidity was described in Chapter 2. Based on the findings from the previous chapter, Chapter 3 presents a first individual participant data analysis conducted on this topic. This new approach enables consistent statistical modelling and unified definitions for surgical outcomes when handling data from multiple international cohorts. Moreover, the relationship between CALI and postoperative infectious complications as shown in Chapter 4 was previously unknown in literature. Additionally, the links between microRNA profiles, complement activation, and the presence of CALI depicted in Chapter 6 and Chapter 7 provided novel insight into the mechanisms underlying its pathological features.

Because addition of bevacizumab to oxaliplatin-based regimens prevents the occurrence of sinusoidal dilatation, which has been demonstrated to be a risk factor for increased postoperative morbidity and diminished tumour response to neoadjuvant chemotherapy, it is advised to add bevacizumab to oxaliplatin-based treatment. Once CALI is diagnosed, treatment for patients with CRLM should be discussed in multidisciplinary meetings and surgical management is advised to be adapted (e.g. apply radiofrequency ablation and two-stage hepatectomy) to prevent the occurrence of morbidity. The role of miR-21 and miR-150 in oxaliplatin-induced sinusoidal dilatation is interesting, however, in view of the small cohort size, further validation in a large cohort is encouraged and warranted. 


\section{References}

1. OCW, Brief valorisatie van onderzoek als taak van de universiteiten; van de bewindslieden Van der Hoeven en Rutte aan de voorzitters van de Colleges van Bestuur van 27 januari 2005 (OWB/Al/0457055).

2. Ferlay J, Soerjomataram I, Dikshit R, Eser S, Mathers C, Rebelo M, et al. Cancer incidence and mortality worldwide: sources, methods and major patterns in GLOBOCAN 2012. Int J Cancer 2015; 136(5):E359-86.

3. Leonard GD, Brenner B, Kemeny NE. Neoadjuvant chemotherapy before liver resection for patients with unresectable liver metastases from colorectal carcinoma. J Clin Oncol 2005; 23(9):2038-48.

4. Kanas GP, Taylor A, Primrose JN, Langeberg WJ, Kelsh MA, Mowat FS, et al. Survival after liver resection in metastatic colorectal cancer: review and meta-analysis of prognostic factors. Clin Epidemiol 2012; 4:283-301.

5. Scheele J. Hepatectomy for liver metastases. Br J Surg 1993; 80(3):274-6.

6. Lam VW, Spiro C, Laurence JM, Johnston E, Hollands MJ, Pleass HC, et al. A systematic review of clinical response and survival outcomes of downsizing systemic chemotherapy and rescue liver surgery in patients with initially unresectable colorectal liver metastases. Ann Surg Oncol 2012; 19(4):1292-301.

7. Adam R, Delvart V, Pascal G, Valeanu A, Castaing D, Azoulay D, et al. Rescue surgery for unresectable colorectal liver metastases downstaged by chemotherapy: a model to predict long-term survival. Ann Surg 2004; 240(4):644-57; discussion 657-8.

8. Rubbia-Brandt L, Audard V, Sartoretti P, Roth AD, Brezault C, Le Charpentier M, et al. Severe hepatic sinusoidal obstruction associated with oxaliplatin-based chemotherapy in patients with metastatic colorectal cancer. Ann Oncol 2004; 15(3):460-6.

9. Rubbia-Brandt L, Lauwers GY, Wang H, Majno PE, Tanabe K, Zhu AX, et al. Sinusoidal obstruction syndrome and nodular regenerative hyperplasia are frequent oxaliplatin-associated liver lesions and partially prevented by bevacizumab in patients with hepatic colorectal metastasis. Histopathology 2010; 56(4):430-9.

10. Vigano L, Ravarino N, Ferrero A, Motta M, Torchio B, Capussotti L. Prospective evaluation of accuracy of liver biopsy findings in the identification of chemotherapy-associated liver injuries. Arch Surg 2012; 147(12):1085-91.

11. Khan AZ, Morris-Stiff G, Makuuchi M. Patterns of chemotherapy-induced hepatic injury and their implications for patients undergoing liver resection for colorectal liver metastases. J Hepatobiliary Pancreat Surg 2009; 16(2):137-44.

12. Vigano L, De Rosa G, Toso C, Andres A, Ferrero A, Roth A, et al. Reversibility of chemotherapy-related liver injury. J Hepatol 2017.

13. Huezo-Diaz Curtis P, Uppugunduri CR, Muthukumaran J, Rezgui MA, Peters C, Bader P, et al. Association of $\mathrm{CTH}$ variant with sinusoidal obstruction syndrome in children receiving intravenous busulfan and cyclophosphamide before hematopoietic stem cell transplantation. Pharmacogenomics J 2016.

14. Hwang DY, Kim SJ, Cheong JW, Kim Y, Jang JE, Lee JY, et al. High pre-transplant serum ferritin and busulfan-thiotepa conditioning regimen as risk factors for hepatic sinusoidal obstructive syndrome after autologous stem cell transplantation in patients with malignant lymphoma. Leuk Lymphoma 2016; 57(1):51-7.

15. Saphner T, Triest-Robertson S, Li H, Holzman P. The association of nonalcoholic steatohepatitis and tamoxifen in patients with breast cancer. Cancer 2009; 115(14):3189-95. 
Acknowledgements 



\section{Acknowledgements}

I would like to thank the China Scholarship Council (CSC), a non-profit institution affiliated to the Ministry of Education of China. CSC financially supported me to do my PhD for 48 months in the Department of Surgery, Maastricht University.

I am grateful to the members of my Assessment Committee: Prof. R. Shiri-Sverdlov (Chair), Prof. G. Beets, Prof. W. Buhre, Prof. H. Grabsch, and Prof. P. Pessaux, for your time and efforts to critically evaluate this thesis.

To conclude the presentation of the work and life during my PhD, I would like to acknowledge all the help and support I have received, and all beautiful memories and experiences I have harvested throughout the time in the Netherlands and abroad (i.e. America, Austria, Belgium, Brazil, Czech Republic, France, Germany, Hungary, Italy, Luxemburg, Slovakia, Sweden, Switzerland, and Vatican). Hereby I would like to address my gratitude and my thanks.

My first gratitude goes to my supervision team. Prof. Steven Olde Damink, dear Steven, my idol, my big boss whom I can talk heart-to-heart and rely on whenever I need. Thanks so much for your enormous supports to me in both research and life. I am amazed by you in various aspects. I was wondering how you managed to handle hundreds of emails per day apart from clinical and research work. After regularly receiving your emails in the very early morning, I seem to find an answer. In the past several years, I have seen our department growing largely since the appointment of you. I admire the open, free, high efficient and fun-filled environment that you created for our department. And I am very impressed by your annually dinner/BBQ party and your super big garden!!! I had wonderful experience of sleeping in the old-fashioned caravan. What is more, thanks so much for your enormous supports on my research projects; and for offering me the oneyear working contract which enable me to not only continue my research line, but also keep staying together with my wife and Zhiqi. Thank you, Steven, I am so lucky to be one of the members in the Surgery department, and I am so proud to be one of your PhD students. Special thanks go to Marja, thanks for lending me your racing bike, with it I extended largely the experience of life in Maastricht, Belgium, and Italy with friends and colleagues, while enjoying the spectacular view beyond the hills and mountains. I also appreciate your generous treat to me and Jieyi in that fancy French restaurant. It was really pleasant to chat with you all the time. 
Prof. Cornelis Dejong, dear Kees, thank you so much for supporting me to apply for the CSC scholarship and accepting me as a member of the department of Surgery. You are always with kind smiles which make me feel so warm. I appreciate that you arranged the semi-monthly progress meeting with me. You are a great listener and wonderful advisor. With your wisdom and clear logic, complicated things always become easier. Thank you for your enormous supports, which are always there whenever I need. Thank you, I had really wonderful years in our department and I am so proud to be one of your students. And I had great memories of our HPB Champagne party!

Dr. Sander Rensen, dear Sander, my daily supervisor, my mentor, my friend. I was lucky enough to have you in my supervision team and be well-surrounded with your patience, help, support, and encouragement in both research and life. Without these, I would have felt so helpless in front of the complicated basic science. Thank you, for taking over me at the moment when I really need you; teaching me lab skills in great detail; and critically commenting on my abstracts, posters, presentations, and manuscripts. Thank you, for your large amount of precious time invested on me. Thank you for everything beyond the words!

Next, I would like to thank the colleagues who are working at the Clinic of the Department of Surgery. Prof. Stassen, although we have talked only for few times, your sense of humour did amaze me. Dear dr. Ronald van Dam, I miss our wonderful time in Sao Paulo and Rio. I really enjoyed our cheers in the party, drinking in the bar, and discussion about the future. Looking forward to seeing you in China in the near future! Dear dr. Mac Bemelmans and dr. Marcel den Dulk, thank you for driving me from Italy to Switzerland for the ALPS meeting, and from Maastricht to Slot Zeist for the IHPBA Dutch Highlight. We had very pleasant talks on the way. Dear Tonneke, Thanks for your warm care when I firstly arrived here. With your thoughtful arrangement for my free accommodation in the hospital guest house, I was able to survive in Maastricht for the first three month with only 1600 euro on hand.

Next, I would like to thank the colleagues who are currently or formerly working in the laboratory of the department of Surgery. Frank, the first impression of you is that you are a serious scientist. Indeed, you do treat science seriously. However, seriousness does not affect the fact that you are a nice person to talk to. Kaatje, a great scientist, from you I learned the precise attitude about research. Livia, the master of Steven's agenda, thanks so much for your arrangements, reminders, signatures, everything visible and invisible that you have done for me. Zita, we had fun together not only in microRNAs, 
but also in Italy. I miss our exhausting bike racing, the food, the wine, and the nice talking.

Mo, my dear former roommate, the master of cell culture and logistics! It was so much pleasant to talk with you all the time and really helpful to have you close by. Hans, Thank you for kindly explaining me the Mass spectrometry. Annemarie, I really appreciate that you even specially translated a PH meter calibrating protocol for me. Thank you so much for your help for doing ELISA. Bas, Thank you so much for helping me with the stainings and PCR. It was a wonderful experience to see your family and taste your collected Whiskies at your place. Chantal and Louise, my new roommates, the future of the lab is yours!

大卫 - 范戴克, David, my dear paranymph, my best and forever Dutch friend, whom I can always rely on and talk heart-to heart. Without you, my life won't be so enjoyable in the Netherlands. I have so many thanks to you: for your efforts to help me integrate into our department, and the Dutch culture; for all those Dutch document translating and the calling around to solve the problems; for the intently listening and the feedbacks; for the confidence that you gave me in the cycling, from which I had great fun in Maastricht, Amsterdam, and Piemont with you guys; for our great memories of suit up Friday, snowboarding, gatherings, cooking, sailing, squash, Mahjong, Chinese chess... Together with you and Sophie, I had a super great New Year 2016 in Sweden, and an unforgettable Chinese new year in my hometown. And I really appreciate that you two travelled for ten thousands of kilometres to join my wedding. We also got so much useful help from you for the baby nursing... Special thanks go to you and Sophie's family, and your grandmother, for the great regards, visiting, and gifts. Thank you for many more supports and helps that go beyond the words!

Frans, my dear friend, my super organized roommate, the soup-master, my paranymph! We started knowing each other from March 2013 at the sushi restaurant in Maastricht. Although not in research, we really had a lot of great memories together, cheese-pot, hot-pot, parties, snowboarding, Chinese dinner at Yong Kee, sailing, one-day in Amsterdam... Thank you so much for all these great memories and experiences you gave; thank you for driving Jieyi and I home from Dusseldorf airport; for cancelling your conference and being my paranymph! I wish you all the best with Celien, be healthy and enjoy your life in this wonderful country!

Audrey, from Efteling to the Chinese dinner at Yong kee, from cycling to Valkenbourg to the one at Pietmont, thank you for giving me so much enjoyable moment together. 
Briete, I am really happy that we are both in the parenthood now. Thank you so much for your suggestion of the stroller and the delicious walnut. Britt, Erik, Marissa, Patrick, Robert, and Sebastiaan, wish you all the best luck for your PhD! Cathy, it was difficult to distinguish you and Magaly at the beginning, but now it's a piece of cake. We had lots of fun when working together on the tissue slicer. Thank you for helping me to sort out hundreds of archived slides from the pathology department. Claire, Cathelijne, Jacqueline, Mirjam, Martijn, and Rianne, thank you for always being so kind to me, for giving me a hand whenever I asked for help. Jasper, thank you for your coffee treat after our lab meeting. It was really great experience of cycling at Piemont with you, you are surprisingly good! Dear Kiran, my roommate, a real boxer, muscle man. It was great to travel with you to Boston, although a bit awkward afterwards that we lost the way when travelling to a pond without GPS. It was great that we learn snowboarding together, always accompany each other on the slope. It was great to talk with you about the future and the life! It was really fun to play with you guys the Chinese chess; and saying the words that only roomies understand the meaning, like "emperty". I wish you and Shareena all the best, be happy, and be loving each other forever. Merel, it was great to know you at ALPS meeting, success in your PhD. Rob, always wearing smart casual in the lab, have fun with your research using the tissue slicer. Romy, congratulations! Rens, what a beautiful name! Yvonne, many congratulations for your wedding, wish you all the best with your beloved. Prof. Ron Heeren, Tiffany and Pierre, thanks so much for your support on the SOS-Mass Spectrometry project. Tom, I was really amazed by your presentation. Thanks so much for visiting and bringing us the delicious Korean Kimba. 刘 红, 刘兄, 你的到来让我倍感亲切! 由于这一年比较忙碌, 非常遗憾没有太多机 会一起玩要。非常感谢你在我和婕一找工作期间的支持和帮助, 也感谢你和嫂子 的热情款待！武汉是个好地方, 期待着将来回国再聚!

Anne-Claire and Jules, it was a lot of fun that we celebrated the New Year together! Anne, we have a lot of great experiences together, the dinner-group, suit and dress-upFriday, Efteling, Chinese dinner. Jules, so cool that we are going to be promoted in the same year. Enjoy your new house! Elwin, Dennis, and Thiemo, congratulations with your new jobs! Irene-fleur, my former roommate, we had so much fun during our cycling at Piemont. Kim, we had great collaboration in research, fruitful two articles published based on this. Apart from research, the life together is also so enjoyable! Sweden, Brazil, Italy, full with great memories in these countries. Success in your career as being a dermatologist! Lori and Martine, thank you for teaching Jieyi skiing at snowworld, we were so excited for the first try-out. Thank you for being so nice to us and Zhiqi. Toine, my roommate, thank you for giving me the great experiences with playing Badminton, 
squash, pooling, and table tennis of course. Victor, great memories with you in Sao Paulo, Rio, and Ilha Grande. Congratulations with your job and success with your PhD!

I would also like to acknowledge all the co-authors in this thesis, without your input, the thesis could not be accomplished. Alison, Celien, and Joanne, thank you for scoring CALI. Bjorn, thank you for your great support of statistics! Margriet and Pamir, thank you for collecting so much clinical data. And all other co-authors, thank you for your contributions!

王萍, 萍姐, 认识你是我的幸运, 能和你做朋友是我的荣幸。感谢你这些年在工 作上的建议和生活上的关照。常常有困惑的时候, 你都能够推己及人, 用你的经 历和睿智去指导。开美, 快乐的小天使, 你发自内心的快乐总能感染我, 和你一 起玩要是一件非常愉快的事情。感谢你带给我们的美好记忆。就像你的名字一 样, 希望你永远快乐! 盛华, 华仔, 睡在我下铺的兄弟! 我的好哥们! 从郑州到 荷兰, 相识、相知、相惜 11 年, 除了缘分还是缘分! 甚至生娃也是你前我后只差 了几个星期！常常回想一起抱娃的场景, 故事感瞬间爆棚！徐青, 怀念和华仔我 们一起连续游三国、一起聚餐、一起三国杀、一起烧烤、一起打麻将、一起包饺 子的时候。感谢我们的微信抱娃群, 经常一起分享育儿经验, 相互扶持, 其乐融 融！衷心祝愿你们在今后一年里博士工作如意, 顺利找到心仪的工作。也特别感 谢阿姨, 万里迢迢来到荷兰看望多米! 饶大夫, 饶老师, 老饶, 在年龄差距面 前, 咱们依然有很多共同话题可以聊。感谢你的信任, 能够和你推心置腹的讨论 工作和生活。怀念一起狂欢节, 一起旅游, 一起聚餐的日子, 希望回国有机会再 聚。张硕, 摄影 “老司机” ，感谢你把我从一窍不通的 “乡下” 带进了摄影的 “省城”。我经常吃惊于你广泛的兴趣, 和对爱好的执着。博士期间一起旅游, 一起聚会, 一起三国杀, 一起打麻将, 一起玩儿摄影, 一起 BBQ, 一起体验你的 Xbox, 留下了太多美好的记忆。希望你和安攀同学继续恩恩爱爱, 相敬如宾, 早 生贵子。于亮, 老于, 于老板。难忘一起去卢森堡, 布鲁日, Valkenburg, 库肯霍 夫玩要的时刻。希望不久回国之后继续一起品啤酒, 一起钓鱼。也恭喜你和梅艳 有情人终成眷属, 期待着你们早日加入 parenthood。正哥和品睿, 羡慜旁人的恩 爱小两口。正哥是大厨, 品睿太有口福, 多次看着正哥做的美食照片流口水。感 
谢你们在我出去开会期间对婕一的关心, 预祝你们在随后的博士答辩中一切顺 利! 辛莹, 在异国他乡, 你就像家人。感谢你带来的无数欢乐和美好记忆; 也感 谢对婕一和致祺的照顾和帮助。也特别感谢阿姨, 不远万里来看望多米! 张明和 天宇, 真的很高兴在马城认识你们, 希望你们在科研的路上一切顺利!

岳父, 没有您的建议和鼓励, 我和婕一就不会萌生出国的念头; 岳母, 感谢您的 信任, 把婕一交给我。感谢你们对我和婕一这个小家无私的付出和支持; 感谢你 们设身处地的替我们考虑。颖儿小妹, 你爱读书的品质像极了你姐姐, 从幼儿园 到初中, 看到你逐渐长大, 生活简单而又充实, 常常让我怀念自己那个年龄段的 日子。希望你继续自己的爱好, 永远健康快乐!

老爸, 老妈, 从 2006 年上大学到现在, 我们聚在一起的日子屈指可数, 甚至一年 见不到一次面。我知道你们为此承受了很多的思念和我不在身边的失落。感谢你 们养育了我, 教会了我做人的准则, 基于此, 我才能在离开你们的日子从容自信 的面对学习、工作上的挑战和生活中的挫折; 感谢你们对我一如既往的鼓励和支 持; 感谢你们对我无私的付出却不求回报; 感谢你们对我的牵挂, 无论我在哪 儿, 你们的牵挂就跟到哪儿。你们在哪儿, 哪儿就是家。老哥, 有个大哥真的是 非常幸福的一件事儿。小时候无忧无虑的, 总想着即使天塌了还有老哥一起顶 着, 也习惯了做你的 “跟屁虫”。实在无法想象假如没有你, 我该有多孤单, 所 以大哥的意义远不止是生活层面的, 还有精神层面的。所以无论何时何地, 大哥 在我心目中的地位都不会改变。大嫂, 嫂子, 这么多年你陪着我哥白手起家, 实 在辛苦。感谢你对老爸、老妈的照顾，感谢你为这个大家庭的付出。金风小妹, 你的到来圆了老爸老妈多年的梦想, 也让这个家变得圆满。很高兴看到你这么独 立有主见, 生活的潚酒从容。希望你一辈子幸福快乐! 还有宇菲、宇程两个小宝 贝, 未来是你们的! 
致祺, 小多米, 你的到来承载着爸爸妈妈的爱, 为我们带来了无限的喜悦。我和 妈妈累并快乐着, 你的一个微笑就能融化爸爸妈妈的心, 让我们把所有的累都抛 在九霄云外。宝贝, 希望你健康快乐的成长, 快点儿长大, 我们好做朋友!

婕一, 亲爱的老婆。感谢命运的安排, 让我在茫茫人海中遇见了你, 一见倾心。 你的出现仿佛透过乌云的那束阳光, 抚在我的心田, 瞬间让我的生活变的无限生 动多彩! 没有你的鼓励, 我不会下定决心出国, 也注定会给将来埋下遗憾。11 年了, 宝贝, 感谢你把自己最青春最珍贵的年华留给了我, 一路陪伴, 相互扶 持; 感谢你小鸟依人外表下那颗强大的心, 在我最无助的时候勇敢的支持着我; 感谢你对我的缺点的包容, 你的勤俭持家; 感谢你所有的默默的付出。我爱你, 老婆, 这辈子只爱你一个! 仅以此书献给你, 我的挚爱! 

Curriculum vitae 



\section{Curriculum vitae}

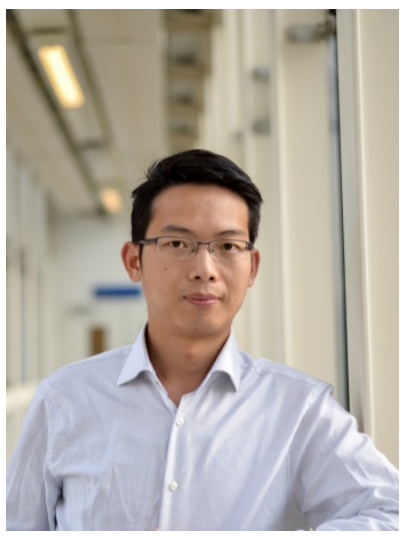

J Zhao was born on the June 18th 1986 in Henan, China. After graduating from the first senior middle school in Queshan (June 2006), he was admitted by Zhengzhou University to study Clinical Medicine (seven-year-joint programme, September 2006).

From September 2006, he started his studies at the School of Basic Medicine at Zhengzhou University for two and half years. He was transferred to the department of Clinical Medicine for clinical curriculums in 2009. From 2010, he started his internship at the first affiliated hospital of Zhengzhou University. From 2011, he started to do his Master at the department of Hepatobilliary and Pancreatic Surgery under the supervision of Prof. Yongfu Zhao. In 2012, he was qualified as a medical practitioner (file no. 01241110412821198606181513). During the period of doing his Master, his interest in scientific research of the liver was sparked. In 2012, he awarded a national grant from the China Scholarship Council (file no. 201207040056). This grant sponsored his fouryear PhD at the department of Surgery of Maastricht University under the supervision of Prof. Steven Olde Damink, Prof. Cornelis Dejong, and Sander Rensen, which resulted in this thesis. In November 2016, with the approval from the supervision team and the China Scholarship Council, he continued his research line at the department of Surgery of Maastricht University, while simultaneously facilitating international collaborations and training as a tutor of problem-based learning. In January 2017, he received an offer from Tongji hospital in Wuhan. With this offer, he will start his training by following the national standardized training protocol in October 2017 and be specialized at Biliary and Pancreatic Surgery in Tongji hospital afterwards. 

List of publications 



\section{List of publications}

\section{Full papers}

Zhao J*, van Mierlo KMC*, Gómez-Ramírez J, Kim H, Pilgrim CHC, Pessaux P, Rensen SS, van der Stok EP, Schaap FG, Soubrane O, Takamoto T, Viganò L, Winkens B, Dejong CHC, Olde Damink SWM; Chemotherapy-associated Liver Injury (CALI) consortium. Systematic review of the influence of chemotherapy-associated liver injury on outcome after partial hepatectomy for colorectal liver metastases. British Journal of Surgery 2017; 104(8):9901002 (*contribute equally)

van Mierlo KMC*, Zhao J*, Kleijnen J, Rensen SS, Schaap FG, Dejong CHC, Olde Damink SWM. The influence of chemotherapy-associated sinusoidal dilatation on short-term outcome after partial hepatectomy for colorectal liver metastases: a systematic review with meta-analysis. Surgical Oncology 2016; 25(3):298-307 (*contribute equally)

Zhao J, Zhao YF. Review of the mechanism underlying hepatic sinusoidal obstruction syndrome. Henan Journal of Surgery 2013; 19(4):81-82

Zhao J, Sawo P, Rouflart MMJ, Winstanley A, Vreuls CPH, Rensen SS, van Mierlo KMC, Lodewick TM, van Woerden V, van Tiel FH, van Dam RM, Dejong CHC, Olde Damink SWM. Impact of chemotherapy-associated liver injury on postoperative infectious complications after partial hepatectomy for patients with colorectal liver metastases. (submitted)

Zhao J, Sawo P, Rouflart MMJ, Winstanley A, Vreuls CPH, Rensen SS, van Mierlo KMC, Lodewick TM, van Woerden V, van Tiel FH, van Dam RM, Dejong CHC, Olde Damink SWM. Impact of chemotherapy-associated liver injury on tumour regression grade and survival after partial hepatectomy for patients with colorectal liver metastases. (submitted)

Zhao J, Rensen SS, Vreuls CPH, van den Broek MA, Driessen A, van Herwijnen M, Jetten MJA, Jennen DGJ, Dejong CHC, Olde Damink SWM. miR-21 and miR-150 downregulation is associated with oxaliplatin-related sinusoidal dilatation and impaired survival. (submitted)

Zhao J, Rensen SS, Vreuls CPH, Driessen A, van den Broek MA, Dejong CHC, Olde Damink SWM. Hepatic complement activation is associated with liver injury in patients with colorectal liver metastases. (submitted) 


\section{Conference abstracts}

Zhao J, van Mierlo KMC, Rensen SS, Schaap FG, Dejong CHC, Olde Damink SWM, on behalf of Chemotherapy-associated Liver Injury (CALI) consortium. The influence of chemotherapy-associated liver injury on outcome after partial hepatectomy for colorectal liver metastases: a systematic review and individual patient data analysis. Journal of Hepatology 2016. Volume 64, issue 2, supplement, page S235. DOI: http://dx.doi.org/10.1016/S0168-8278(16)00227-0

Zhao J, Rensen SS, Vreuls CPH, van den Broek MA, Driessen A, van Herwijnen M, Jetten MJA, Jennen DGJ, Dejong CHC, Olde Damink SWM. miR-21 and miR-150 downregulation is associated with oxaliplatin-related sinusoidal obstruction syndrome and impaired survival. Journal of Hepatology 2016. Volume 64, issue 2, supplement, page S567. DOI: http://dx.doi.org/10.1016/S0168-8278(16)01027-8

Zhao J, van Mierlo KMC, Rensen SS, Schaap FG, Dejong CHC, Olde Damink SWM, on behalf of Chemotherapy-associated Liver Injury (CALI) consortium. The influence of chemotherapy-associated liver injury on outcome after partial hepatectomy for colorectal liver metastases: Results from a multicentre cohort study. HPB 2016. Volume 18, supplement 1, page e58. DOI: http://dx.doi.org/10.1016/j.hpb.2016.02.142

Zhao J, Rensen SS, Vreuls CPH, van den Broek MA, Jetten MJA, Coonen M, Jennen DGJ, van Herwijnen M, Dejong CHC, Olde Damink SWM. miR-21 and miR-150 downregulation correlates with oxaliplatin-related sinusoidal obstruction syndrome and impaired survival. HPB 2016. Volume 18, supplement 1, page e135. DOI: http://dx.doi.org/10.1016/j.hpb.2016.02.318

Zhao J, Sawo P, Rouflart MMJ, Rensen SS, van Mierlo KMC, Lodewick TM, van Woerden $\mathrm{V}$, van Tiel FH, van Dam RM, Dejong CHC, Olde Damink SWM. Postoperative infectious complications decrease long-term survival after partial hepatic resection for colorectal liver metastases. HPB 2016. Volume 18, supplement 1, page e228. DOI: http://dx.doi.org/10.1016/j.hpb.2016.02.569 


\section{Scientific activities}

\section{Conference oral presentations}

2017 (2) ALPS Liver and Pancreatic Surgery Meeting 2017 (Madonna di Campiglio, Italy) Impact of chemotherapy-associated liver injury on tumour regression grade and survival in patients with colorectal liver metastases

2016 (4) 12th World Congress of the International Hepato-Pancreato Biliary Association (IHPBA, Sao Paulo, Brazil) The influence of chemotherapy-associated liver injury on outcome after partial hepatectomy for colorectal liver metastases: a systematic review and individual patient data analysis

2015 (11) 12th International Medical Postgraduate Conference (Prague, Czech Republic) miR-21 and miR-150 downregulation is associated with oxaliplatin-related sinusoidal obstruction syndrome and impaired survival

2015 (10) Dutch Society for Gastroenterology (NVGE, Veldhoven, the Netherlands) Postoperative infectious complications decrease long-term survival after partial hepatic resection for colorectal liver metastases

2015 (10) Dutch Society for Gastroenterology (NVGE, Veldhoven, the Netherlands) The influence of chemotherapy-associated liver injury on outcome after partial hepatectomy for colorectal liver metastases: systematic review and pooled analysis of individual patient data

2014 (10) Dutch Society for Gastroenterology (NVGE, Veldhoven, the Netherlands) Oxaliplatin related sinusoidal obstruction syndrome is correlated with down-regulation of miR-21 and miR-150

\section{Conference posters}

2016 (4) 12th World Congress of the International Hepato-Pancreato Biliary Association (IHPBA, Sao Paulo, Brazil) miR-21 and miR-150 downregulation is associated with oxaliplatin-related sinusoidal obstruction syndrome and impaired survival

2016 (4) 12th World Congress of the International Hepato-Pancreato Biliary Association (IHPBA, Sao Paulo, Brazil) Postoperative infectious complications decrease long-term survival after partial hepatic resection for colorectal liver metastases 
2016 (4) The International Liver Congress 2016 (EASL, Barcelona, Spain) miR-21 and miR150 downregulation is associated with oxaliplatin-related sinusoidal obstruction syndrome and impaired survival

2016 (4) The International Liver Congress 2016 (EASL, Barcelona, Spain) The influence of chemotherapy-associated liver injury on outcome after partial hepatectomy for colorectal liver metastases: a systematic review and individual patient data analysis

2016 (3) Dutch Society for Gastroenterology (NVGE, Veldhoven, the Netherlands) Complement activation in patients with chemotherapy-associated steatosis and steatohepatitis 